\title{
Gold-Catalyzed Cyclization of 2-Alkynylaldehyde Cyclic Acetals via Hydride Shift for the Synthesis of Indenone Derivatives
}

Tsuyoshi Yamada, ${ }^{\text {a }}$ Kwihwan Park, ${ }^{\text {a }}$ Takumu Tachikawa, ${ }^{a}$ Akiko Fujii, ${ }^{\text {a }}$ Matthias Rudolph, ${ }^{\mathrm{b}}$ A. Stephen K. Hashmi, ${ }^{\text {b and Hironao Sajiki*a }}$

${ }^{a}$ Laboratory of Organic Chemistry, Gifu Pharmaceutical University, 1-25-4 Daigaku-nishi, Gifu 501-1196, Japan

${ }^{\mathrm{b}}$ Organisch-Chemisches Institut, Heidelberg University, Im Neuenheimer Feld 270, 69120 Heidelberg, Germany

\section{Contents}

\section{General information}

2. Optimization of the reaction conditions for the gold-catalyzed cyclization reaction

3. Mechanistical investigations

4. Deprotection of 2-(p-tolyl)-1H-inden-1-one (2b)

5. General procedures for the syntheses of 2-alkynylaldehyde cyclic acetals and the gold-catalyzed cyclization reactions

6. Procedures for the synthesis of substrates

7. Procedures for the synthesis of products

8. HMBC and NOESY NMR spectra

9. ${ }^{1} \mathrm{H},{ }^{2} \mathrm{H}$ and ${ }^{13} \mathrm{C}$ NMR spectra of stating materials and products

10. References

\section{General information}

All reagents and solvents were obtained from commercial sources and used without further purification unless otherwise noted. Flash column chromatography was performed with Silica Gel $60 \mathrm{~N}$ (Kanto Chemical Co., Inc., 63-210 $\mu \mathrm{m}$ spherical, neutral). ${ }^{1} \mathrm{H}$ and ${ }^{13} \mathrm{C}$ NMR spectra were recorded on a JEOL ECZ $400\left({ }^{1} \mathrm{H}: 400 \mathrm{MHz},{ }^{13} \mathrm{C}: 100 \mathrm{MHz},{ }^{2} \mathrm{H}: 61 \mathrm{MHz}\right)$ or ECA 500 spectrometer $\left({ }^{1} \mathrm{H}: 500 \mathrm{MHz},{ }^{13} \mathrm{C}: 125 \mathrm{MHz},{ }^{2} \mathrm{H}: 77 \mathrm{MHz}\right)$ at room temperature in $\mathrm{CDCl}_{3}$ as a solvent and an internal standard ( ${ }^{1} \mathrm{H}$ NMR: $\delta=0.00$ for $\mathrm{CDCl}_{3} ;{ }^{13} \mathrm{C}$ NMR: $\delta=77.0$ for $\mathrm{CDCl}_{3}$ ). IR spectra were recorded by a Brucker FT-IR ALPHA. ESI high resolution mass spectra (HRMS) were measured by a Shimadzu hybrid IT-TOF mass spectrometer. Melting points were measured by a SANSYO SMP-300 melting point apparatus. Elementary analysis were measured by a J-SCIENCE LAB JM11. 
2. Optimization of the reaction conditions for the gold-catalyzed cyclization reaction

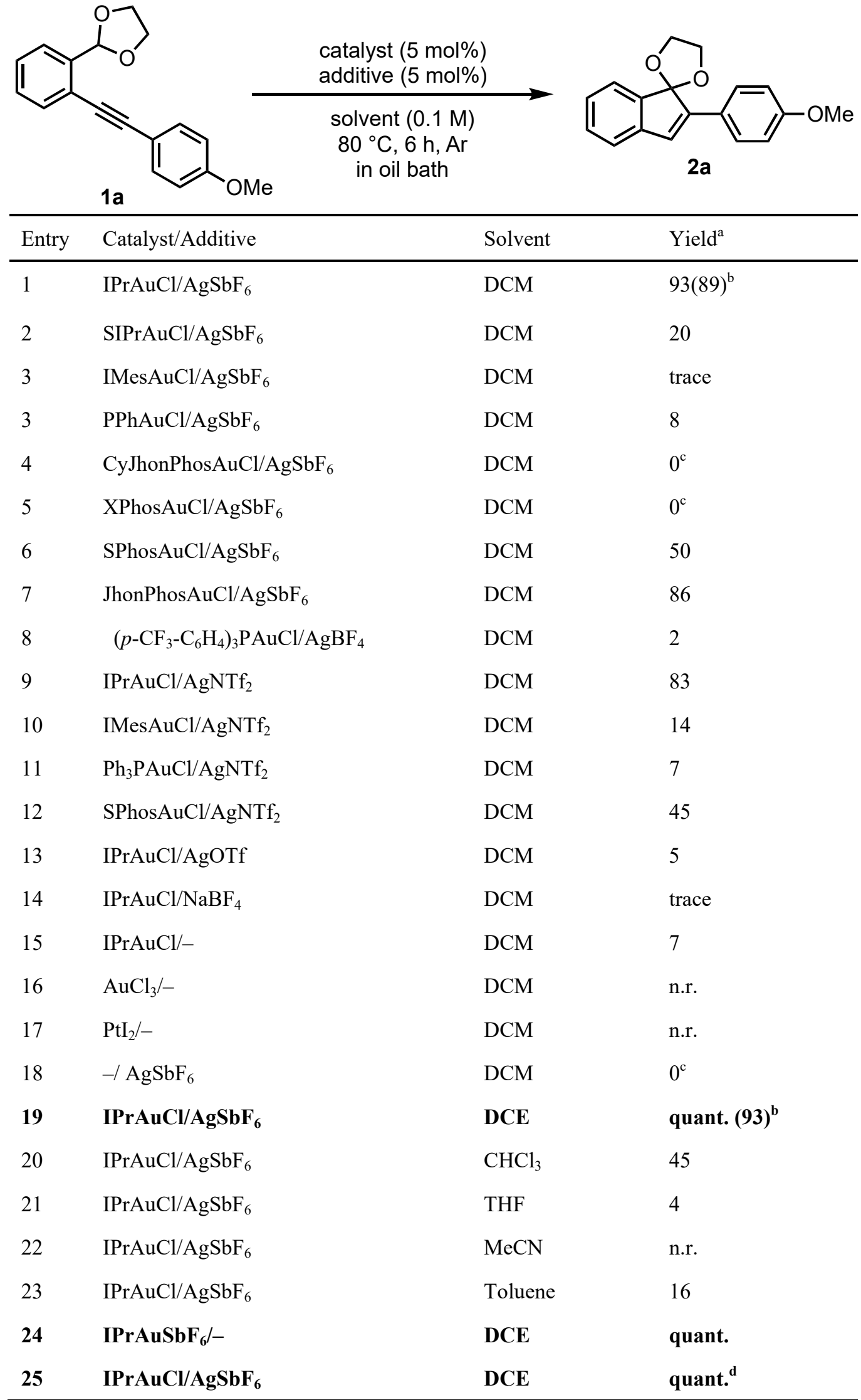

${ }^{a}$ Yield was determined by ${ }^{1} \mathrm{H}$ NMR using 1,1,2,2-tetrachloroethane as an internal standard. ${ }^{\mathrm{b}}$ Isolated yield. ${ }^{\mathrm{c}}$ starting material was completely consumed. ${ }^{\mathrm{d}} 1 \mathrm{~mol} \%$ of $\mathrm{IPrAuCl}$ and $\mathrm{AgSbF}_{6}$ were used. 


\section{Mechanistical investigations}

\section{Isotope labeling experiments}

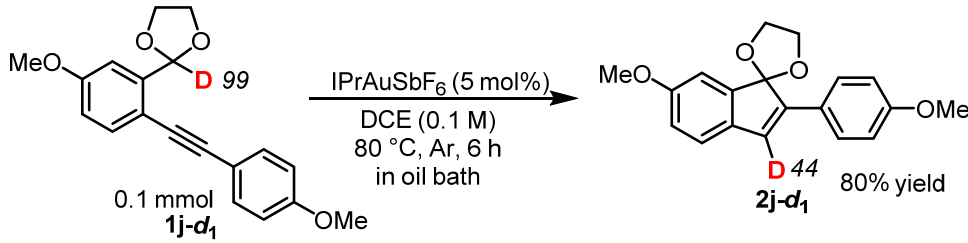
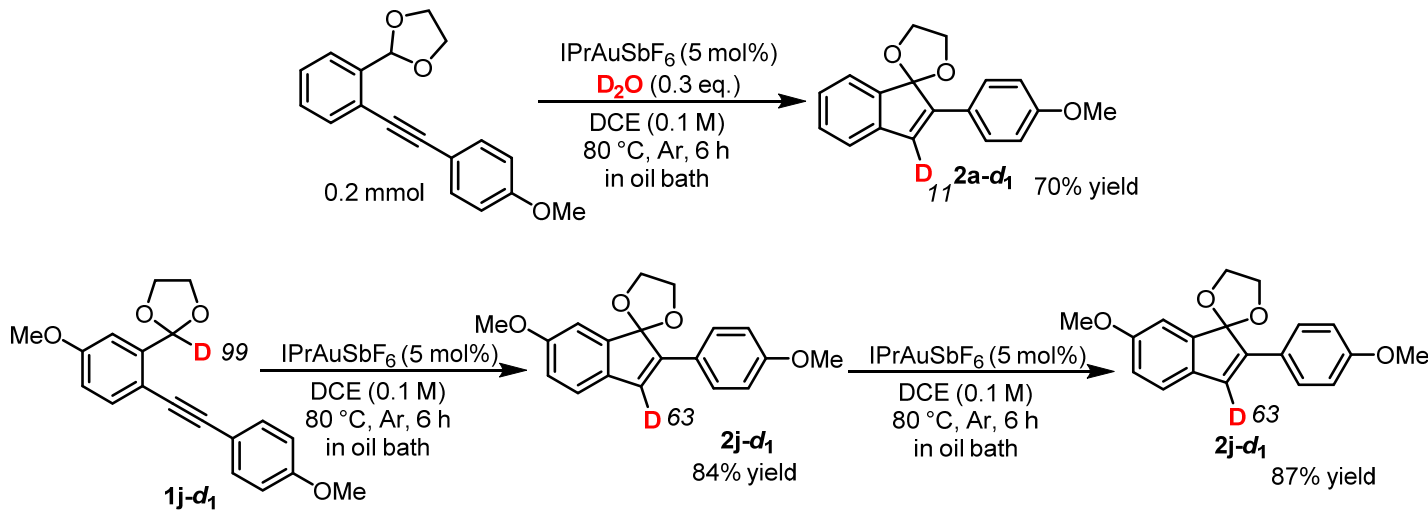

\section{Crossover experiments}
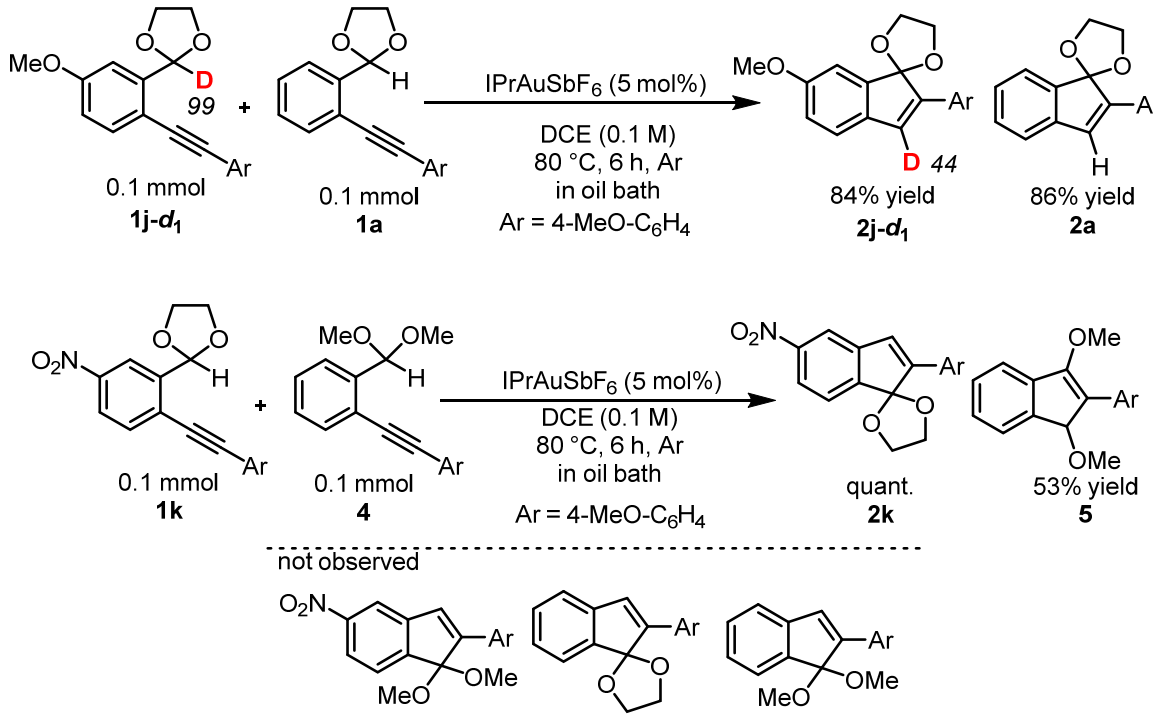

Synthesis of 2-allyl-2-(2-((4-methoxyphenyl)ethynyl)phenyl)-1,3-dioxolane (12)
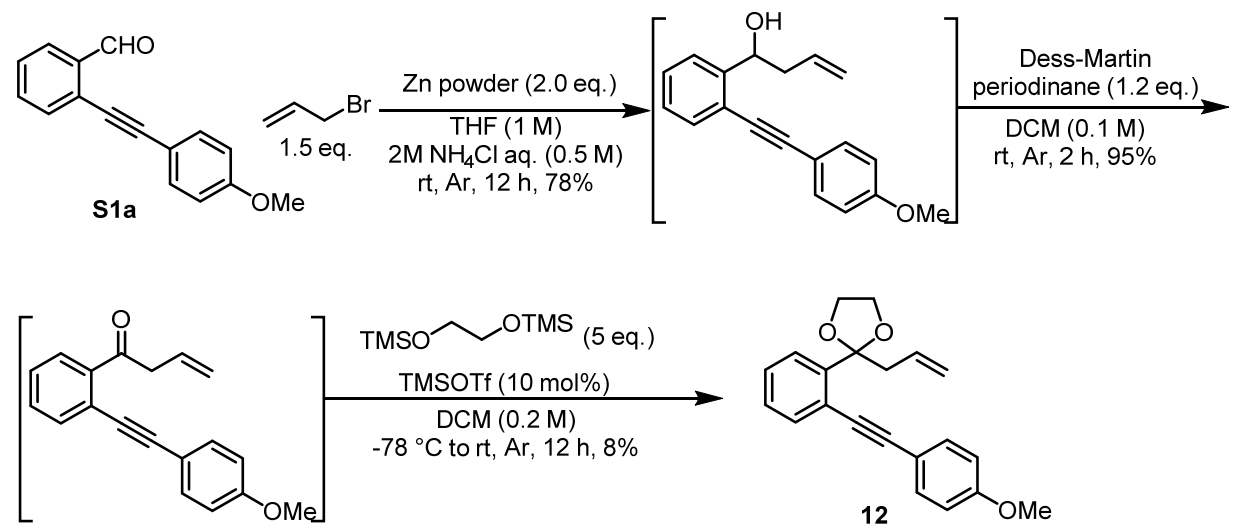

2-Allyl-2-(2-((4-methoxyphenyl)ethynyl)phenyl)-1,3-dioxolane (12) 
<smiles>C=CCC1(c2ccccc2C#Cc2ccc(OC)cc2)OCCO1</smiles>

${ }^{1} \mathrm{H}$ NMR $\left(500 \mathrm{MHz}, \mathrm{CDCl}_{3}\right): \delta 7.59-7.55(\mathrm{~m}, 2 \mathrm{H}), 7.50(\mathrm{~d}, J=9.0 \mathrm{~Hz}, 2 \mathrm{H}), 7.29-7.25(\mathrm{~m}, 2 \mathrm{H}), 6.89(\mathrm{~d}, J=9.0 \mathrm{~Hz}, 2 \mathrm{H})$, 5.85-5.77 (m, 1H), 5.10-5.04 (m, 2H), 4.10-4.03 (m, 2H), 3.86-3.82 (m 5H), 3.08 (d, J=7.0 Hz, 2H); ${ }^{13} \mathrm{C}$ NMR $(125 \mathrm{MHz}$, $\left.\mathrm{CDCl}_{3}\right): \delta 159.6,143.0,133.8,132.9,132.8,127.8,127.4,126.4,121.1,117.9,115.9,113.9,109.8,94.1$ 87.7, 64.7, 55.3, 42.7; IR (ATR) $\mathrm{cm}^{-1}: 3072,2956,2888,2836,2214,1685,1641,1605,1568,1509,1464,1441,1287,1245,1203,1172$, 1105, 1030; ESI-HRMS m/z: $343.1305\left([\mathrm{M}+\mathrm{Na}]^{+}\right)$; Calcd. for $\mathrm{C}_{21} \mathrm{H}_{20} \mathrm{O}_{3} \mathrm{Na}: 343.1305$.

\section{Gold-catalyzed cyclization reaction using 12 as a substrate}

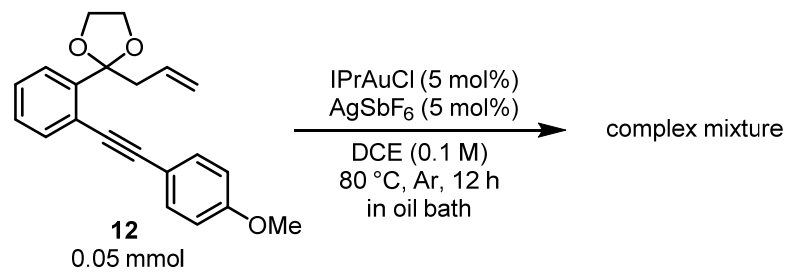

\section{Addition of olefins or sulfoxide}
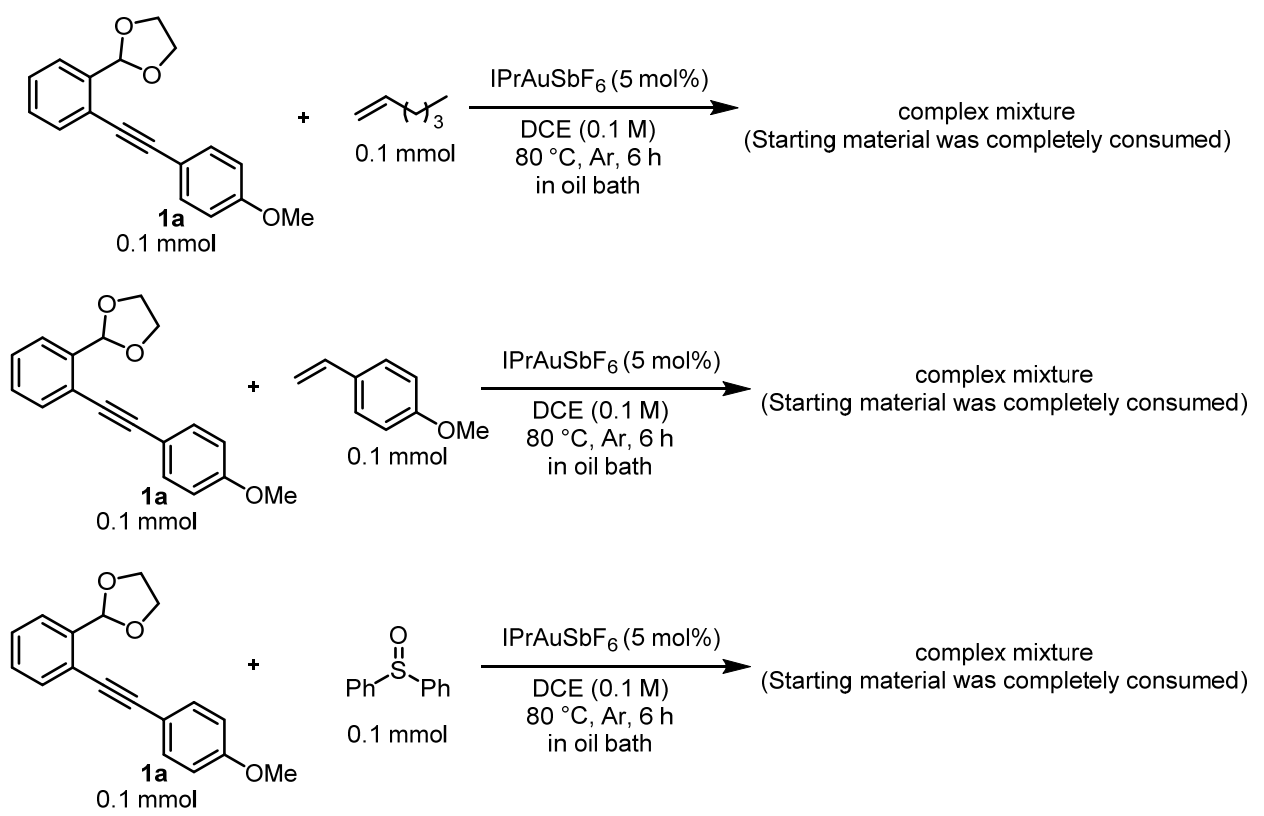

\section{Deprotection of 2-(p-tolyl)-1H-inden-1-one (2b)}

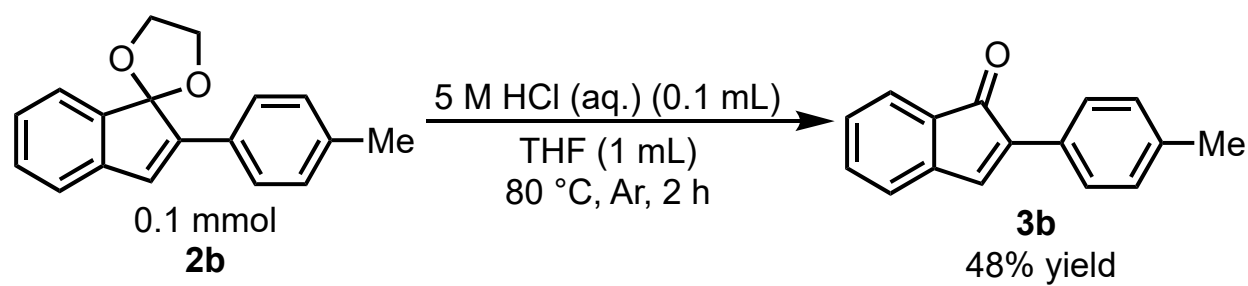

To a solution of 2'-(p-tolyl)spiro[[1,3]dioxolane-2,1'-indene] $(\mathbf{2 b}, 26.4 \mathrm{mg}, 0.10 \mathrm{mmol})$ in THF $(1 \mathrm{~mL})$ was added $5 \mathrm{M}$ aqueous $\mathrm{HCl}(0.1 \mathrm{~mL})$ at room temperature under argon. The reaction mixture was stirred at $70{ }^{\circ} \mathrm{C}$ in oil bath for $2 \mathrm{~h}$. To the reaction mixture was added saturated $\mathrm{NaHCO}_{3}$ aqueous solution at room temperature and extracted with ethyl acetate. The 
combined organic layers were dried over $\mathrm{Na}_{2} \mathrm{SO}_{4}$ and concentrated in vacuo. The residue was purified by silica-gel column chromatography (hexane / ethyl acetate $=40 / 1)$ to give 2-( $p$-tolyl)-1H-inden-1-one $(\mathbf{3 b}, 10.5 \mathrm{mg}, 0.48 \mathrm{mmol})$ in $48 \%$ yield as a red solid.

MP: $62-65^{\circ} \mathrm{C} ;{ }^{1} \mathrm{H}$ NMR $\left(400 \mathrm{MHz}, \mathrm{CDCl}_{3}\right): \delta 7.70(\mathrm{~d}, J=7.6 \mathrm{~Hz}, 2 \mathrm{H}), 7.59(\mathrm{~s}, 1 \mathrm{H}), 7.45(\mathrm{~d}, J=6.8 \mathrm{~Hz}, 1 \mathrm{H}), 7.34(\mathrm{dd}, J=$ 7.2, 7.2 Hz, 1H), 7.23-7.19 (m, 3H), 7.06 (d, $J=7.2 \mathrm{~Hz}, 1 \mathrm{H}), 2.38$ (s 3H); ${ }^{13} \mathrm{C}$ NMR (100 MHz, $\mathrm{CDCl}_{3}$ ): $\delta 197.2,144.1$, 141.6, 138.6, 136.3, 134.1, 131.2, 129.3, 128.5, 128.5, 127.2, 123.0, 121.8, 21.4; IR (ATR) $\mathrm{cm}^{-1}: 3025,2920,2852,1704$, 1598, 1506, 1454, 1365, 1324, 1292, 1259, 1167, 1094, 1031; ESI-HRMS m/z: $221.0952\left([\mathrm{M}+\mathrm{H}]^{+}\right)$; Calcd. for $\mathrm{C}_{16} \mathrm{H}_{13} \mathrm{O}$ : 221.0961.

5. General procedures for the syntheses of 2-alkynylaldehyde cyclic acetals and the gold-catalyzed cyclization reactions

General procedure A (GP A): Sonogashira coupling reactions<smiles>O=Cc1ccccc1Br</smiles>

1 eq.<smiles>[R]C#C</smiles>

1.0-1.2 eq.

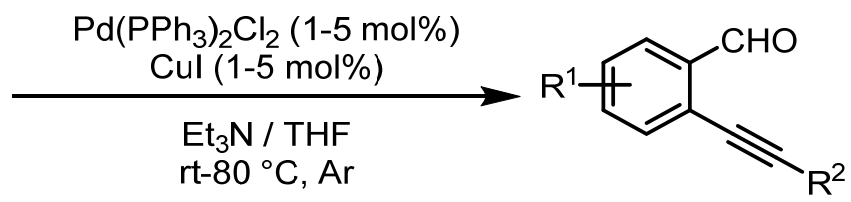

A solution of aryl halide (1.0 eq.), alkyne (1.0-1.2 eq.), $\mathrm{Pd}\left(\mathrm{PPh}_{3}\right)_{2} \mathrm{Cl}_{2}(1-5 \mathrm{~mol} \%)$ and $\mathrm{CuI}(1-5 \mathrm{~mol} \%)$ in a mixture of $\mathrm{Et}_{3} \mathrm{~N}$ and THF was stirred at room temperature- $80{ }^{\circ} \mathrm{C}$ in oil bath until complete conversion was detected by TLC analysis. The crude reaction mixture was diluted with ethyl acetate and filtered through celite pad, concentrated, and purified by silica-gel column chromatography.

General procedure B (GP B): acetal protections of aldehydes

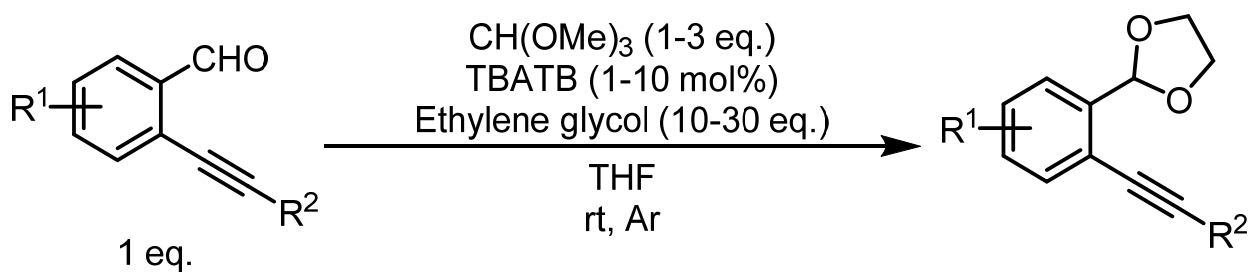

A solution of aldehyde (1.0 eq.), ethylene glycol (10-30 eq.), $\mathrm{CH}(\mathrm{OMe})_{3}(1-3$ eq.) and tetrabutylammonium tribrime (TBATB, 1-10 mol\%) in THF was stirred at room temperature until complete conversion was detected by TLC analysis. The crude reaction mixture was diluted with ethyl acetate, washed with saturated $\mathrm{NaHCO}_{3}$ aqueous solution, concentrated, and purified by silica-gel column chromatography.

General procedure C (GP C): gold-catalyzed cyclization reactions of 2-alkynyl aldehyde cyclic acetals<smiles>[R]C#CC1=C(C2OCCO2)C=C[R]C=C1</smiles>
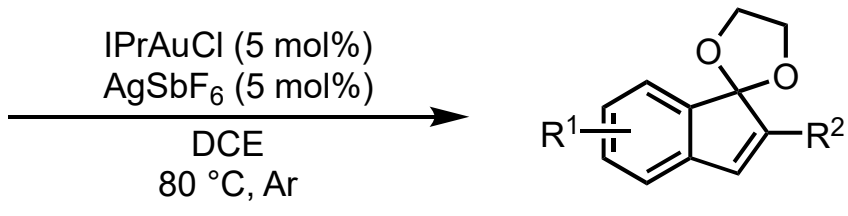

In a $9 \mathrm{~mL}$ vial, 2-alkynyl aldehyde cyclic acetal (1.0 eq.), $\operatorname{IPrAuCl}(5 \mathrm{~mol} \%)$ and $\mathrm{AgSbF}_{6}(5 \mathrm{~mol} \%)$ were added in dichloroethane. The reaction mixture was heated $80^{\circ} \mathrm{C}$ in oil bath upon completion. The reaction mixture is cooled at room 
temperature, quenched with $\mathrm{Et}_{3} \mathrm{~N}(0.1 \mathrm{~mL})$, filterd through aluminium silicate with $\mathrm{Et}_{2} \mathrm{O}$, concentrated, and purified by silica-gel column chromatography.

\section{Procedures for the synthesis of substrates}

2-((4-Methoxyphenyl)ethynyl)benzaldehyde (S1a)

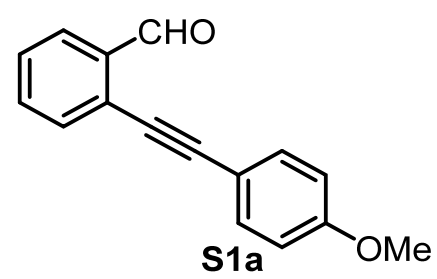

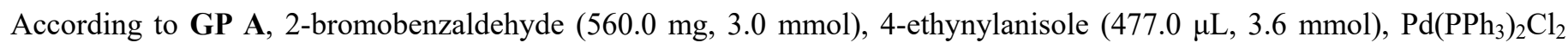
(63.2 mg, $90.0 \mu \mathrm{mol}), \mathrm{CuI}(17.1 \mathrm{mg}, 90.0 \mu \mathrm{mol}), \mathrm{Et}_{3} \mathrm{~N}(6 \mathrm{~mL})$ and THF $(9 \mathrm{~mL})$ were stirred for $2 \mathrm{~h}$ at $50{ }^{\circ} \mathrm{C}$ in oil bath. Purification by flash silica-gel column chromatography (hexane / ethyl acetate $=15 / 1$ ) to give 2-((4-methoxyphenyl)ethynyl)benzaldehyde (S1a, $496.2 \mathrm{mg}, 2.1 \mathrm{mmol})$ in 70\% yield as an orangish solid.

${ }^{1} \mathrm{H}$ NMR (400 MHz, $\left.\mathrm{CDCl}_{3}\right): \delta 10.65(\mathrm{~s}, 1 \mathrm{H}), 7.94(\mathrm{dd}, J=1.0,7.8 \mathrm{~Hz}, 1 \mathrm{H}), 7.63-7.55(\mathrm{~m}, 2 \mathrm{H}), 7.51$ (d. $\left.J=8.6 \mathrm{~Hz}, 2 \mathrm{H}\right)$, $7.45-7.41(\mathrm{~m}, 1 \mathrm{H}), 6.91(\mathrm{~d}, J=8.6 \mathrm{~Hz}, 2 \mathrm{H}), 3.85(\mathrm{~s}, 3 \mathrm{H})$. Spectroscopic data of ${ }^{1} \mathrm{H}$ NMR was identical to that of the reference 1 .

2-(2-((4-Methoxyphenyl)ethynyl)phenyl)-1,3-dioxolane (1a)

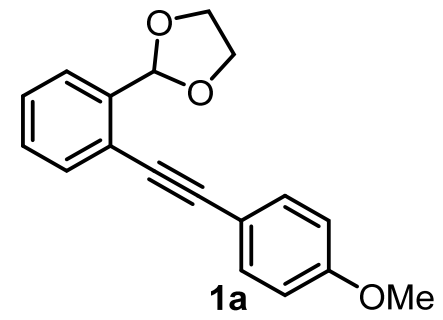

According to GP B, 2-((4-methoxyphenyl)ethynyl)-benzaldehyde (S1a, $708.8 \mathrm{mg}, 3.0 \mathrm{mmol})$, ethylene glycol (1.7 mL, $30.0 \mathrm{mmol}), \mathrm{CH}(\mathrm{OMe})_{3}(495.0 \mu \mathrm{L}, 4.5 \mathrm{mmol})$, TBATB $(14.4 \mathrm{mg}, 30.0 \mu \mathrm{mol})$ and THF $(2 \mathrm{~mL})$ were stirred for $24 \mathrm{~h}$ at room temperature. Purification by flash silica-gel column chromatography (hexane / ethyl acetate $=10 / 1$ ) to give 2-(2-((4-methoxyphenyl)ethynyl)phenyl)-1,3-dioxolane (1a, $540.8 \mathrm{mg}, 1.9 \mathrm{mmol})$ in 64\% yield as a colorless solid. MP: $69-72{ }^{\circ} \mathrm{C} ;{ }^{1} \mathrm{H}$ NMR $\left(500 \mathrm{MHz}, \mathrm{CDCl}_{3}\right): \delta 7.61-7.50(\mathrm{~m}, 1 \mathrm{H}), 7.55-7.53(\mathrm{~m}, 1 \mathrm{H}), 7.48(\mathrm{~d}, J=8.8 \mathrm{~Hz}, 1 \mathrm{H}), 7.37-7.32$ $(\mathrm{m}, 2 \mathrm{H}), 6.89(\mathrm{~d}, J=8.8 \mathrm{~Hz}, 2 \mathrm{H}), 6.29(\mathrm{~s}, 1 \mathrm{H}), 4.23-4.17(\mathrm{~m}, 2 \mathrm{H}), 4.12-4.05(\mathrm{~m}, 2 \mathrm{H}), 3.83(\mathrm{~s}, 3 \mathrm{H}) ;{ }^{13} \mathrm{C} \mathrm{NMR}(125 \mathrm{MHz}$, $\left.\mathrm{CDCl}_{3}\right): \delta 159.7,138.7,133.0,132.3,129.0,128.1,126.1,122.8,115.3,113.9,102.1,94.0,85.3,65.5,55.3$; IR (ATR) $\mathrm{cm}^{-1}$ : 3066, 2955, 2931, 2843, 2720, 2213, 2047, 1934, 1601, 1567, 1510, 1451, 1432, 1395, 1367, 1340, 1287, 1279, 1273, 1248, 1232, 1206, 1174, 1147, 1104, 1085, 1028, 1004; ESI-HRMS m/z: $303.1008\left([\mathrm{M}+\mathrm{Na}]^{+}\right)$; Calcd. for $\mathrm{C}_{18} \mathrm{H}_{16} \mathrm{O}_{3} \mathrm{Na}: 303.0992$.

\section{2-(p-Tolylethynyl)benzaldehyde (S1b)}

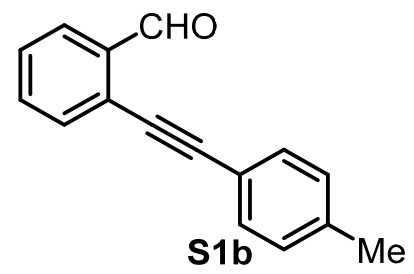

According to GP A, 2-bromobenzaldehyde (925.1 mg, $5.0 \mathrm{mmol}$ ), 4-ethynyltoluene (694.4 mg, $\left.6.0 \mathrm{mmol}), \mathrm{Pd}_{\left(\mathrm{PPh}_{3}\right.}\right)_{2} \mathrm{Cl}_{2}$ (175.5 mg, $250.0 \mu \mathrm{mol}), \mathrm{CuI}(47.6 \mathrm{mg}, 250.0 \mu \mathrm{mol})$ and $\mathrm{Et}_{3} \mathrm{~N}(80 \mathrm{~mL})$ were stirred for $3 \mathrm{~h}$ at $50{ }^{\circ} \mathrm{C}$ in oil bath. Purification 
by flash silica-gel column chromatography (hexane / ethyl acetate $=15 / 1)$ to give 2 -(p-tolylethynyl)benzaldehyde (S1b,745.9 mg, $3.4 \mathrm{mmol}$ ) in 68\% yield as an orangish solid.

${ }^{1} \mathrm{H}$ NMR (400 MHz, $\left.\mathrm{CDCl}_{3}\right): \delta 10.65(\mathrm{~s}, 1 \mathrm{H}), 7.94(\mathrm{~d}, J=7.2 \mathrm{~Hz}, 1 \mathrm{H}), 7.64-7.55(\mathrm{~m}, 2 \mathrm{H}), 7.47-7.41(\mathrm{~m}, 3 \mathrm{H}), 7.19(\mathrm{~d}, J=$ $6.8 \mathrm{~Hz}, 2 \mathrm{H}), 2.39$ (s, 3H). Spectroscopic data of ${ }^{1} \mathrm{H}$ NMR was identical to that of the reference 2.

\section{2-(2-(p-Tolylethynyl)phenyl)-1,3-dioxolane (1b)}<smiles>Cc1ccc(C#Cc2ccccc2C2OCCO2)cc1</smiles>

According to GP B, 2-(p-tolylethynyl)benzaldehyde (S1b, $745.0 \mathrm{mg}, 3.4 \mathrm{mmol})$, ethylene glycol (7.5 mL, $135.0 \mathrm{mmol})$, $\mathrm{CH}(\mathrm{OMe})_{3}(1.5 \mathrm{~mL}, 13.5 \mathrm{mmol})$, TBATB $(65.2 \mathrm{mg}, 68.0 \mu \mathrm{mol})$ and $\mathrm{THF}(2 \mathrm{~mL})$ were stirred for $4 \mathrm{~h}$ at room temperature. Purification by flash silica-gel column chromatography (hexane / ethyl acetate $=15 / 1$ ) to give 2-(2-(p-tolylethynyl)phenyl)-1,3-dioxolane (528.6 mg, $2.0 \mathrm{mmol})$ in 60\% yield as an colorless solid.

MP: $100-101{ }^{\circ} \mathrm{C} ;{ }^{1} \mathrm{H}$ NMR $\left(400 \mathrm{MHz}, \mathrm{CDCl}_{3}\right): \delta 7.61-7.57(\mathrm{~m}, 1 \mathrm{H}), 7.56-7.52(\mathrm{~m}, 1 \mathrm{H}), 7.43(\mathrm{~d}, J=8.0 \mathrm{~Hz}, 2 \mathrm{H})$, 7.38-7.32 (m, 2H), $7.16(\mathrm{~d}, J=8.0 \mathrm{~Hz}, 2 \mathrm{H}), 6.28(\mathrm{~s}, 1 \mathrm{H}), 4.24-4.16(\mathrm{~m}, 2 \mathrm{H}), 4.12-4.04(\mathrm{~m}, 2 \mathrm{H}), 2.37(\mathrm{~s}, 3 \mathrm{H}) ;{ }^{13} \mathrm{C} \mathrm{NMR}$ $\left(100 \mathrm{MHz}, \mathrm{CDCl}_{3}\right): \delta 138.8,138.6,132.5,131.5,129.1,129.0,128.3,126.2,122.7,120.1,102.1,94.1,85.9,65.6,21.5 ; \mathrm{IR}$ (ATR) $\mathrm{cm}^{-1}: 2954,2880,2754,2215,1916,1661,1958,1571,1508,1484,1450,1388,1307,1271,1229,1204,1183,1166$, 1147, 1104, 1067, 1016; ESI-HRMS m/z: $265.1226\left([\mathrm{M}+\mathrm{H}]^{+}\right)$; Calcd. for $\mathrm{C}_{18} \mathrm{H}_{17} \mathrm{O}_{2}: 265.1223$.

\section{2-(Phenylethynyl)benzaldehyde (S1c)}

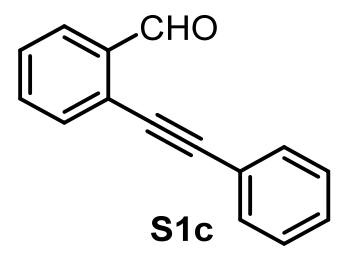

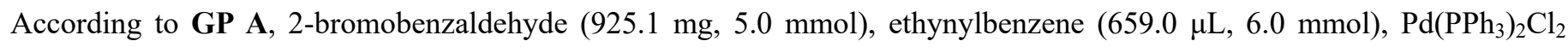
(175.5 mg, $250.0 \mu \mathrm{mol})$, $\mathrm{CuI}(47.6 \mathrm{mg}, 250.0 \mu \mathrm{mol})$ and $\mathrm{Et}_{3} \mathrm{~N}(80 \mathrm{~mL})$ were stirred for $3 \mathrm{~h}$ at $50{ }^{\circ} \mathrm{C}$ in oil bath. Purification by flash silica-gel column chromatography (hexane / ethyl acetate $=15 / 1$ ) to give 2-(phenylethynyl)-benzaldehyde (S1c, $629.0 \mathrm{mg}, 3.1 \mathrm{mmol}$ ) in $61 \%$ yield as a colorless solid.

${ }^{1} \mathrm{H}$ NMR (400 MHz, CDCl $): \delta 10.66(\mathrm{~s}, 1 \mathrm{H}), 7.96(\mathrm{~d}, J=7.6 \mathrm{~Hz}, 1 \mathrm{H}), 7.66-7.56(\mathrm{~m}, 4 \mathrm{H}), 7.46(\mathrm{t}, J=7.6 \mathrm{~Hz}, 1 \mathrm{H})$, 7.40-7.39 (m, 3H). Spectroscopic data of ${ }^{1} \mathrm{H}$ NMR was identical to that of the reference 2.

\section{2-(2-(phenylethynyl)phenyl)-1,3-dioxolane (1c)}<smiles>C(#Cc1ccccc1C1OCCO1)c1ccccc1</smiles>

To a solution of 2-(phenylethynyl)benzaldehyde (S1c, $309.4 \mathrm{mg}, 1.50 \mathrm{mmol})$ and ethylene glycol $(836.0 \mu \mathrm{L}, 15.0 \mathrm{mmol})$ in toluene $(5 \mathrm{~mL})$ was added $p$ - $\mathrm{TsOH}(103.3 \mathrm{mg}, 0.6 \mathrm{mmol})$ at room temperature under argon. The reaction mixture was stirred 
at $130{ }^{\circ} \mathrm{C}$ in oil bath for $24 \mathrm{~h}$. To the reaction mixture was added $\mathrm{NaHCO}_{3}$ aq. at room temperature and extracted with ethyl acetate. The combined organic layers were dried over $\mathrm{Na}_{2} \mathrm{SO}_{4}$ and concentrated in vacuo. The residue was purified by silica-gel column chromatography (hexane / ethyl acetate $=20 / 1)$ to give of 2-(2-(phenylethynyl)phenyl)-1,3-dioxolane (1c, $268.2 \mathrm{mg}, 1.1 \mathrm{mmol}$ ) in $72 \%$ yield as an colorless solid.

${ }^{1} \mathrm{H}$ NMR (400 MHz, $\left.\mathrm{CDCl}_{3}\right): \delta 7.61-7.52(\mathrm{~m}, 4 \mathrm{H}), 7.40-7.33$ (m, 5H), $6.29(\mathrm{~s}, 1 \mathrm{H}), 4.24-4.16(\mathrm{~m}, 2 \mathrm{H}), 4.12-4.04(\mathrm{~m}, 2 \mathrm{H})$. Spectroscopic data of ${ }^{1} \mathrm{H}$ NMR was identical to that of the reference 3 .

\section{2-((4-Fluorophenyl)ethynyl)benzaldehyde (S1d)}

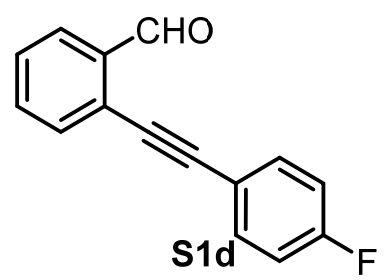

According to GP A, 2-bromobenzaldehyde (555.1 mg, $3.0 \mathrm{mmol})$, 4-ethynylfluorobenzene $(374.0 \mu \mathrm{L}, 3.3 \mathrm{mmol})$, $\mathrm{Pd}\left(\mathrm{PPh}_{3}\right)_{2} \mathrm{Cl}_{2}(63.2 \mathrm{mg}, 90.0 \mu \mathrm{mol}), \mathrm{CuI}(17.1 \mathrm{mg}, 90.0 \mu \mathrm{mol}), \mathrm{Et}{ }_{3} \mathrm{~N}(3 \mathrm{~mL})$ and THF $(6 \mathrm{~mL})$ were stirred for $6 \mathrm{~h}$ at $80{ }^{\circ} \mathrm{C}$ in oil bath. Purification by flash silica-gel column chromatography (hexane / ethyl acetate $=15 / 1$ ) to give of 2-((4-fluorophenyl)ethynyl)benzaldehyde (S1d, $363.9 \mathrm{mg}, 1.6 \mathrm{mmol})$ in 54\% yield as an orangish oil.

${ }^{1} \mathrm{H}$ NMR (400 MHz, $\left.\mathrm{CDCl}_{3}\right): \delta 10.63(\mathrm{~s}, 1 \mathrm{H}), 7.96(\mathrm{~d}, J=7.6 \mathrm{~Hz}, 1 \mathrm{H}), 7.65-7.54(\mathrm{~m}, 4 \mathrm{H}), 7.47(\mathrm{dd}, J=7.6,7.6 \mathrm{~Hz}, 1 \mathrm{H})$, 7.11-7.07 (m, 2H). Spectroscopic data of ${ }^{1} \mathrm{H}$ NMR was identical to that of the reference 2.

\section{2-(2-((4-Fluorolphenyl)ethynyl)phenyl)-1,3-dioxolane (1d)}

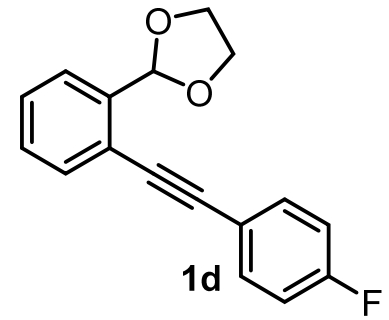

According to GP B, 2-((4-fluorophenyl)ethynyl)benzaldehyde (S1d, $336.3 \mathrm{mg}, 1.50 \mathrm{mmol}$ ), ethylene glycol (837.0 $\mu \mathrm{L}, 15.0$ $\mathrm{mmol}), \mathrm{CH}(\mathrm{OMe})_{3}(247.0 \mu \mathrm{L}, 2.3 \mathrm{mmol})$, TBATB $(7.2 \mathrm{mg}, 15.0 \mu \mathrm{mol})$ and THF $(2 \mathrm{~mL})$ were stirred for $24 \mathrm{~h}$ at room temperature. Purification by flash silica-gel column chromatography (hexane / ethyl acetate $=10 / 1$ ) to give 2-(2-((4-fluorophenyl)ethynyl)phenyl)-1,3-dioxolane (1d, $245.5 \mathrm{mg}, 0.9 \mathrm{mmol}) \mathrm{in} 61 \%$ yield as a yellow oil.

${ }^{1} \mathrm{H}$ NMR (400 MHz, $\left.\mathrm{CDCl}_{3}\right): \delta 7.60(\mathrm{~d}, J=7.2 \mathrm{~Hz}, 1 \mathrm{H}), 7.56-7.49(\mathrm{~m}, 3 \mathrm{H}), 7.40-7.33(\mathrm{~m}, 2 \mathrm{H}), 7.07-7.03(\mathrm{~m}, 2 \mathrm{H}), 6.27$ (s, $1 \mathrm{H}), 4.24-4.15(\mathrm{~m}, 2 \mathrm{H}), 4.13-4.05(\mathrm{~m}, 2 \mathrm{H}) ;{ }^{13} \mathrm{C} \mathrm{NMR}\left(100 \mathrm{MHz}, \mathrm{CDCl}_{3}\right): \delta 162.6$ (d, J=248.2 Hz), 138.9, $133.5(\mathrm{~d}, J=$ 8.6 Hz), 132.5, 129.0, 128.5, 126.2, 122.3, $119.3(\mathrm{~d}, J=2.9 \mathrm{~Hz}), 115.6(\mathrm{~d}, J=22.0 \mathrm{~Hz}), 102.0,92.8,86.3,65.6$; IR (ATR) $\mathrm{cm}^{-1}: 2954,2886,1597,1505,1449,1393,1272,1222,1155,1108,1093,1069,1027,969,942,834,798,756,664,626$, 558, 527, 510, 499 ; ESI-HRMS m/z: $269.0956\left([\mathrm{M}+\mathrm{H}]^{+}\right)$; Calcd. for $\mathrm{C}_{17} \mathrm{H}_{14} \mathrm{O}_{2} \mathrm{~F}: 269.0972$.

\section{Methyl 4-((2-folmylphenyl)ethynyl)benzoate (S1e)}

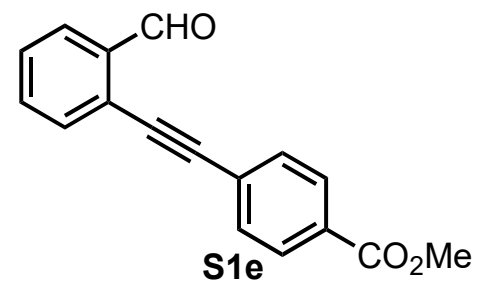


According to GP A, 2-bromobenzaldehyde (925.1 mg, $5.0 \mathrm{mmol})$, methyl 4-ethynylbenzoate (880.9 $\mathrm{mg}, 5.5 \mathrm{mmol})$, $\mathrm{Pd}\left(\mathrm{PPh}_{3}\right)_{2} \mathrm{Cl}_{2}(105.3 \mathrm{mg}, 150.0 \mu \mathrm{mol}), \mathrm{CuI}(28.6 \mathrm{mg}, 150.0 \mu \mathrm{mol}), \mathrm{Et}_{3} \mathrm{~N}(6 \mathrm{~mL})$ and THF $(9 \mathrm{~mL})$ were stirred for $6 \mathrm{~h}$ at $80{ }^{\circ} \mathrm{C}$ in oil bath. Purification by flash silica-gel column chromatography (hexane / ethyl acetate $=10 / 1$ ) to give methyl 4-((2-folmylphenyl)ethynyl)benzoate (S1e, $1057.1 \mathrm{mg}, 4.0 \mathrm{mmol})$ in $80 \%$ yield as a pale yellow solid.

${ }^{1} \mathrm{H}$ NMR (400 MHz, $\mathrm{CDCl}_{3}$ ): $\delta 10.64(\mathrm{~s}, 1 \mathrm{H}), 8.06(\mathrm{~d}, J=8.8 \mathrm{~Hz}, 2 \mathrm{H}), 8.02(\mathrm{~d}, J=8.8 \mathrm{~Hz}, 1 \mathrm{H}), 7.97(\mathrm{dd}, J=0.8,8.0 \mathrm{~Hz}$, $1 \mathrm{H}), 7.69-7.59(\mathrm{~m}, 4 \mathrm{H}), 7.50(\mathrm{t}, J=8.0 \mathrm{~Hz}, 1 \mathrm{H}), 3.95(\mathrm{~s}, 3 \mathrm{H})$. Spectroscopic data of ${ }^{1} \mathrm{H}$ NMR was identical to that of the reference 2 .

Methyl 4-((2-(1,3-dioxolan-2-yl)phenyl)ethynyl)benzoate (1e)

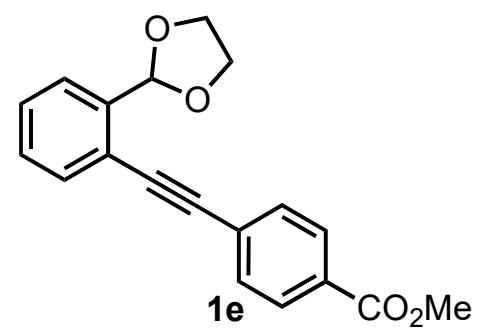

According to GP B, methyl 4-((2-folmylphenyl)ethynyl)benzoate (S1e, $792.8 \mathrm{mg}, 3.0 \mathrm{mmol})$, ethylene glycol (1.7 mL, 30.0 $\mathrm{mmol}), \mathrm{CH}(\mathrm{OMe})_{3}(492.0 \mu \mathrm{L}, 4.5 \mathrm{mmol})$, TBATB $(72.3 \mathrm{mg}, 150.0 \mu \mathrm{mol})$ and THF $(2 \mathrm{~mL})$ were stirred for $24 \mathrm{~h}$ at room temperature. Purification by flash silica-gel column chromatography (hexane / ethyl acetate $=10 / 1$ ) to give methyl 4-((2-(1,3-dioxolan-2-yl)phenyl)ethynyl)benzoate (1e, $666.0 \mathrm{mg}, 2.2 \mathrm{mmol})$ in $72 \%$ yield as a colorless solid.

MP: 95-96 ${ }^{\circ} \mathrm{C} ;{ }^{1} \mathrm{H}$ NMR $\left(400 \mathrm{MHz}, \mathrm{CDCl}_{3}\right): \delta 8.03(\mathrm{~d}, J=8.4 \mathrm{~Hz}, 2 \mathrm{H}), 7.63-7.56(\mathrm{~m}, 4 \mathrm{H}), 7.43-7.35(\mathrm{~m}, 2 \mathrm{H}), 6.28(\mathrm{~s}, 1 \mathrm{H})$, 4.24-4.16 (m, 2H), 4.12-4.06 (m, 2H), 3.93 (s, 3H); $\left.{ }^{13} \mathrm{C} \mathrm{NMR} \mathrm{(100} \mathrm{MHz,} \mathrm{CDCl}_{3}\right): \delta 166.5,139.2,132.7,131.5,129.6$, $129.5,129.1,129.0,127.9,126.3,121.9,102.0,93.1,89.6,65.6,52.2$; IR (ATR) $\mathrm{cm}^{-1}: 2952,2901,2216,1711,1601,1509$, 1476, 1448, 1433, 1392, 1309, 1273, 1205, 1191, 1173, 1148, 1104, 1064, 1018; ESI-HRMS m/z: 309.1115 ([M+H] $\left.{ }^{+}\right)$; Calcd. for $\mathrm{C}_{19} \mathrm{H}_{17} \mathrm{O}_{4}: 309.1121$.

2-(o-Tolylethynyl)benzaldehyde (S1f)

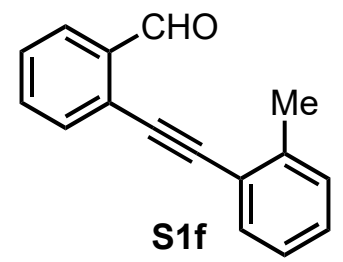

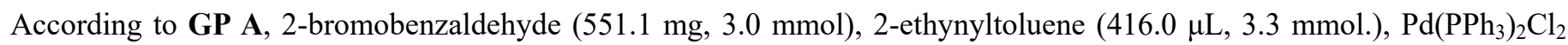
$(105.3 \mathrm{mg}, 150.0 \mu \mathrm{mol}), \mathrm{CuI}(28.6 \mathrm{mg}, 150.0 \mu \mathrm{mol}), \mathrm{Et}_{3} \mathrm{~N}(5 \mathrm{~mL})$ and THF $(10 \mathrm{~mL})$ were stirred for $2 \mathrm{~h}$ at $50{ }^{\circ} \mathrm{C}$ in oil bath. Purification by flash silicagel column chromatography (hexane / ethyl acetate $=25 /$ 1) to give 2-(o-tolylethynyl)benzaldehyde (S1f, $654.2 \mathrm{mg}, 3.0 \mathrm{mmol}$ ) in $99 \%$ yield as a pale yellow oil.

${ }^{1} \mathrm{H}$ NMR $\left(400 \mathrm{MHz}, \mathrm{CDCl}_{3}\right): \delta 10.68(\mathrm{~s}, 1 \mathrm{H}), 7.96(\mathrm{dd}, J=1.2,7.6 \mathrm{~Hz}, 1 \mathrm{H}), 7.67-7.65(\mathrm{~m}, 1 \mathrm{H}), 7.60$ (ddd, $J=1.2,7.6,7.6$ $\mathrm{Hz}, 1 \mathrm{H}), 7.54(\mathrm{~d}, J=7.2 \mathrm{~Hz}, 1 \mathrm{H}), 7.46(\mathrm{dd}, J=7.6,7.6 \mathrm{~Hz}, 1 \mathrm{H}), 7.32-7.26(\mathrm{~m}, 2 \mathrm{H}), 7.23-7.19(\mathrm{~m}, 1 \mathrm{H}), 2.54(\mathrm{~s}, 3 \mathrm{H})$. Spectroscopic data of ${ }^{1} \mathrm{H}$ NMR was identical to that of the reference 2.

2-(2-(o-Tolylethynyl)phenyl)-1,3-dioxolane (1f) 


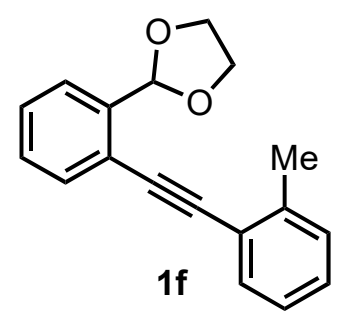

According to GP B, 2-(o-tolylethynyl)-benzaldehyde (S1f, $330.4 \mathrm{mg}, 1.5 \mathrm{mmol})$, ethylene glycol (839.0 $\mu \mathrm{L}, 15.0 \mathrm{mmol})$, $\mathrm{CH}(\mathrm{OMe})_{3}(246.0 \mu \mathrm{L}, 2.3 \mathrm{mmol})$, TBATB $(7.2 \mathrm{mg}, 15.0 \mu \mathrm{mol})$ and THF $(2 \mathrm{~mL})$ were stirred for $10 \mathrm{~h}$ at room temperature. Purification by flash silica-gel column chromatography (hexane / ethyl acetate = 25 / 1) to give 2-(2-(o-tolylethynyl)phenyl)-1,3-dioxolane (1f, $274.8 \mathrm{mg}, 1.0 \mathrm{mmol}$ ) in 69\% yield as a yellow oil.

${ }^{1} \mathrm{H}$ NMR (400 MHz, $\left.\mathrm{CDCl}_{3}\right): \delta 7.65-7.61(\mathrm{~m}, 1 \mathrm{H}), 7.59-7.55(\mathrm{~m}, 1 \mathrm{H}), 7.51(\mathrm{~d}, J=7.2 \mathrm{~Hz}, 1 \mathrm{H}), 7.40-7.33(\mathrm{~m}, 2 \mathrm{H})$, 7.26-7.24 (m, 2H), 7.21-7.15 (m, 1H), $6.31(\mathrm{~s}, 1 \mathrm{H}), 4.24-4.03(\mathrm{~m}, 4 \mathrm{H}), 2.52(\mathrm{~s}, 3 \mathrm{H}) ;{ }^{13} \mathrm{C} \mathrm{NMR}\left(100 \mathrm{MHz}, \mathrm{CDCl}_{3}\right): \delta 140.2$, 138.6, 132.4, 131.9, 129.5, 129.0, 128.4, 128.4, 125.9, 125.5, 123.0, 122.8, 102.0, 93.0, 90.4, 65.5, 20.7; IR (ATR) $\mathrm{cm}^{-1}$ : 3061, 2949, 2883, 2212, 1600, 1571, 1491, 1452, 1391, 1272, 1203, 1115, 1101, 1068, 1028; ESI-HRMS m/z: 287.1037 $\left([\mathrm{M}+\mathrm{Na}]^{+}\right)$; Calcd. for $\mathrm{C}_{18} \mathrm{H}_{16} \mathrm{O}_{2} \mathrm{Na}: 287.1043$.

2-((6-Methoxynaphthalen-2-yl)ethynyl)benzaldehyde (S1g)

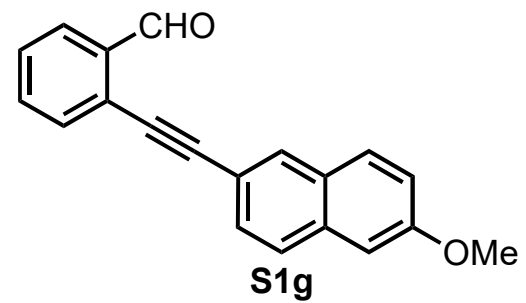

According to GP A, 2-bromobenzaldehyde (740.1 mg, $4.0 \mathrm{mmol})$, 2-ethynyl-6-methoxynapthalene (801.8 mg, $4.4 \mathrm{mmol})$, $\mathrm{Pd}\left(\mathrm{PPh}_{3}\right)_{2} \mathrm{Cl}_{2}(84.2 \mathrm{mg}, 120.0 \mu \mathrm{mol}), \mathrm{CuI}(22.9 \mathrm{mg}, 120.0 \mu \mathrm{mol}), \mathrm{Et}_{3} \mathrm{~N}(6 \mathrm{~mL})$ and $\mathrm{THF}(9 \mathrm{~mL})$ were stirred for $6 \mathrm{~h}$ at $80{ }^{\circ} \mathrm{C}$ in oil bath. Purification by flash silica-gel column chromatography (hexane / ethyl acetate = 10 / 1) to give 2-((6-methoxynaphthalen-2-yl)ethynyl)benzaldehyde (S1g, $436.3 \mathrm{mg}, 1.5 \mathrm{mmol})$ in 38\% yield as a pale yellow solid.

${ }^{1} \mathrm{H}$ NMR (400 MHz, $\left.\mathrm{CDCl}_{3}\right): \delta 10.72(\mathrm{~s}, 1 \mathrm{H}), 8.03(\mathrm{~s}, 1 \mathrm{H}), 7.97(\mathrm{dd}, J=1.0,7.8 \mathrm{~Hz}, 1 \mathrm{H}), 7.74(\mathrm{dd}, J=2.6,8.9 \mathrm{~Hz}, 2 \mathrm{H})$, $7.68(\mathrm{dd}, J=1.0,7.8 \mathrm{~Hz}, 1 \mathrm{H}), 7.63-7.56(\mathrm{~m}, 2 \mathrm{H}), 7.46(\mathrm{dd}, J=7.6,7.6 \mathrm{~Hz}, 1 \mathrm{H}), 7.19$ (dd, 2.6, 8.9 Hz, $1 \mathrm{H}), 7.14(\mathrm{~d}, 2.6 \mathrm{~Hz}$, 1H), 3.95 (s, 3H). Spectroscopic data of ${ }^{1} \mathrm{H}$ NMR was identical to that of the reference 4.

2-(2-((6-Methoxynaphthalen-2-yl)ethynyl)phenyl)-1,3-dioxolane (1g)

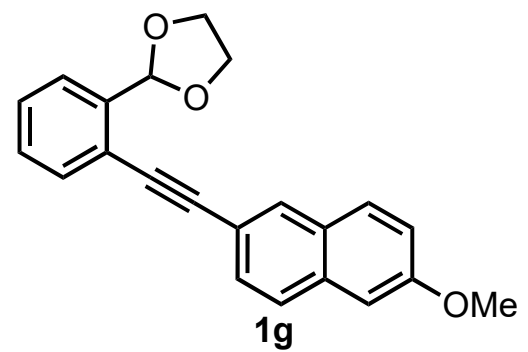

According to GP B, 2-((6-methoxynaphthalen-2-yl)ethynyl)benzaldehyde (S1g, $286.3 \mathrm{mg}, 1.0 \mathrm{mmol})$, ethylene glycol (1.1 $\mathrm{mL}, 20.0 \mathrm{mmol}), \mathrm{CH}(\mathrm{OMe})_{3}(164.0 \mu \mathrm{L}, 1.5 \mathrm{mmol})$, TBATB $(4.8 \mathrm{mg}, 10.0 \mu \mathrm{mol})$ and THF $(5 \mathrm{~mL})$ were stirred for $24 \mathrm{~h}$ at room temperature. Purification by flash silica-gel column chromatography (hexane / ethyl acetate $=10 / 1$ ) to give 2-(2-((6-methoxynaphthalen-2-yl)ethynyl)phenyl)-1,3-dioxolane (1g, $251.2 \mathrm{mg}, 760.0 \mu \mathrm{mol})$ in $76 \%$ yield as a colorless 
solid.

MP: 97-98 ${ }^{\circ} \mathrm{C} ;{ }^{1} \mathrm{H}$ NMR (400 MHz, $\left.\mathrm{CDCl}_{3}\right): \delta 7.98(\mathrm{~s}, 1 \mathrm{H}), 7.71(\mathrm{dd}, J=8.4,8.4 \mathrm{~Hz}, 2 \mathrm{H}), 7.63-7.54(\mathrm{~m}, 3 \mathrm{H}), 7.40-7.33$ (m, 2H), $7.16(\mathrm{dd}, J=2.4,9.2 \mathrm{~Hz}, 1 \mathrm{H}), 7.12(\mathrm{~d}, J=2.4 \mathrm{~Hz}, 1 \mathrm{H}), 6.35$ (s, 1H), 4.26-4.18 (m, 2H), 4.15-4.09 (m, 2H), 3.93 (s, 3H); ${ }^{13} \mathrm{C}$ NMR $\left(100 \mathrm{MHz}, \mathrm{CDCl}_{3}\right): \delta 158.3,138.8,134.2,132.5,131.2,129.4,129.0,129.0,128.4,128.3,126.8,126.2$, 122.7, 119.4, 118.1, 105.8, 102.1, 94.5, 86.2, 65.6, 55.3; IR (ATR) $\mathrm{cm}^{-1}: 2970,2885,2052,1597,1457,1452,1400,1385$, 1255, 1204, 1163, 1141, 1099, 1086, 1064, 1021; ESI-HRMS m/z: $353.1132\left([\mathrm{M}+\mathrm{Na}]^{+}\right)$; Calcd. for $\mathrm{C}_{22} \mathrm{H}_{18} \mathrm{O}_{3} \mathrm{Na}: 353.1148$.

\section{2-(2-(3-Thienyl)ethynyl)benzaldehyde (S1h)}

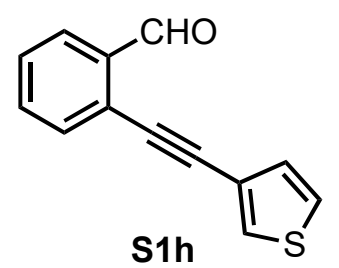

According to GP A, 1-bromo-2-folmyl-1-cyclohexene $104.5 \mathrm{mg}, 0.6 \mathrm{mmol})$, 3-bromothiophene (57.0 $\mu \mathrm{L}, 0.6 \mathrm{mmol}$ ), $\mathrm{Pd}\left(\mathrm{PPh}_{3}\right)_{2} \mathrm{Cl}_{2}(12.6 \mathrm{mg}, 18.0 \mu \mathrm{mol}), \mathrm{CuI}(3.4 \mathrm{mg}, 18.0 \mu \mathrm{mol}), \mathrm{Et}_{3} \mathrm{~N}(1 \mathrm{~mL})$ and $\mathrm{THF}(2 \mathrm{~mL})$ were stirred for $12 \mathrm{~h}$ at $80{ }^{\circ} \mathrm{C}$ in oil bath. Purification by flash silica-gel column chromatography (hexane / ethyl acetate $=10 / 1$ ) to give 2-(2-(3-thienyl)ethynyl)benzaldehyde (S1h, $72.2 \mathrm{mg}, 280.0 \mu \mathrm{mol})$ in $47 \%$ yield as a red oil.

${ }^{1} \mathrm{H}$ NMR (400 MHz, $\left.\mathrm{CDCl}_{3}\right): \delta 10.62(\mathrm{~s}, 1 \mathrm{H}), 7.95(\mathrm{~d}, J=7.6 \mathrm{~Hz}, 1 \mathrm{H}), 7.64-7.56(\mathrm{~m}, 3 \mathrm{H}), 7.45(\mathrm{dd}, J=7.6,7.6 \mathrm{~Hz}, 1 \mathrm{H})$, $7.35(\mathrm{dd}, J=3.2,5.2 \mathrm{~Hz}, 1 \mathrm{H}), 7.23(\mathrm{dd}, J=1.2,5.2 \mathrm{~Hz}, 1 \mathrm{H})$. Spectroscopic data of ${ }^{1} \mathrm{H}$ NMR was identical to that of the reference 13 .

2-(2-(Thiophen-3-ylethynyl)phenyl)-1,3-dioxolane (1h)<smiles>C(#Cc1ccccc1C1OCCO1)c1ccsc1</smiles>

According to GP B, 2-(2-(3-thienyl)ethynyl)benzaldehyde (S1h, $636.8 \mathrm{mg}, 3.0 \mathrm{mmol})$, ethylene glycol (3.3 mL, $60.0 \mathrm{mmol})$, $\mathrm{CH}(\mathrm{OMe})_{3}(492 \mathrm{mg}, 4.5 \mathrm{mmol})$, TBATB $(43.4 \mathrm{mg}, 90.0 \mu \mathrm{mol})$ and THF $(2 \mathrm{~mL})$ were stirred for $3 \mathrm{~h}$ at room temperature. Purification by flash silica-gel column chromatography (hexane / ethyl acetate $=10 / 1$ ) to give 2-(2-(thiophen-3-ylethynyl)phenyl)-1,3-dioxolane (1h, $512.6 \mathrm{mg}, 2.0 \mathrm{mmol})$ in 67\% yield as a red oil.

${ }^{1} \mathrm{H}$ NMR $\left(400 \mathrm{MHz}, \mathrm{CDCl}_{3}\right): \delta 7.60-7.53(\mathrm{~m}, 3 \mathrm{H}), 7.39-7.30(\mathrm{~m}, 3 \mathrm{H}), 7.20(\mathrm{dd}, J=0.8,4.4 \mathrm{~Hz}, 1 \mathrm{H}), 6.26(\mathrm{~s}, 1 \mathrm{H})$, 4.24-4.16 (m, 2H), 4.12-4.04 (m, 2H); ${ }^{13} \mathrm{C}$ NMR (100 MHz, $\left.\mathrm{CDCl}_{3}\right): \delta 138.8,132.5,129.8,129.0,128.7,128.4,126.2$, 125.3, 122.4, 122.2, 102.1, 89.0, 86.1, 65.6; IR (ATR) $\mathrm{cm}^{-1}: 3104,2952,2883,2208,1599,1571,1523,1480,1449,1390$, 1356, 1267, 1209, 1136, 1107, 1067, 1023; ESI-HRMS m/z: $257.0640\left([\mathrm{M}+\mathrm{H}]^{+}\right)$; Calcd. for $\mathrm{C}_{15} \mathrm{H}_{13} \mathrm{O}_{2} \mathrm{~S}: 257.0631$.

\section{2-((4-Methoxyphenyl)ethynyl)-5-methylbenzaldehyde (S1i)}

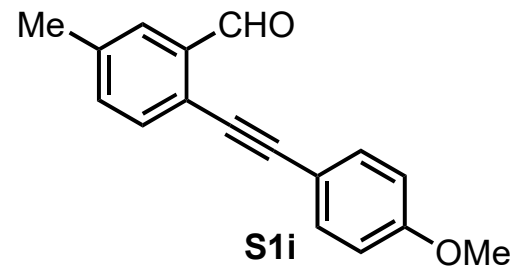

According to GP A, 2-bromo-5-methylbenzaldehyde (790.2 mg, $4.0 \mathrm{mmol})$, 4-ethynylanisole $(570.0 \mu \mathrm{L}, 4.4 \mathrm{mmol})$, 
$\mathrm{Pd}\left(\mathrm{PPh}_{3}\right)_{2} \mathrm{Cl}_{2}(140.4 \mathrm{mg}, 200.0 \mu \mathrm{mol}), \mathrm{CuI}(38.1 \mathrm{mg}, 200.0 \mu \mathrm{mol}), \mathrm{Et}_{3} \mathrm{~N}(4 \mathrm{~mL})$ and THF $(6 \mathrm{~mL})$ were stirred for $5 \mathrm{~h}$ at $50{ }^{\circ} \mathrm{C}$ in oil bath. Purification by flash silica-gel column chromatography (hexane / ethyl acetate $=15 / 1$ ) to give 2-((4-methoxyphenyl)ethynyl)-5-methylbenzaldehyde (S1i, $892.4 \mathrm{mg}, 2.8 \mathrm{mmol})$ in $71 \%$ yield as a pale yellow solid.

${ }^{1} \mathrm{H}$ NMR $\left(400 \mathrm{MHz}, \mathrm{CDCl}_{3}\right): \delta 10.62(\mathrm{~s}, 1 \mathrm{H}), 7.75(\mathrm{~s}, 1 \mathrm{H}), 7.56-7.47(\mathrm{~m}, 3 \mathrm{H}), 7.39(\mathrm{~d}, J=8.0,1 \mathrm{H}), 6.90(\mathrm{~d}, J=8.8 \mathrm{~Hz}$, 2H), 3.85 (s, 3H), 2.42 (s, 3H). Spectroscopic data of ${ }^{1} \mathrm{H}$ NMR was identical to that of the reference 5.

\section{2-(2-((4-Methoxyphenyl)ethynyl)-5-methylphenyl)-1,3-dioxolane (1i)}

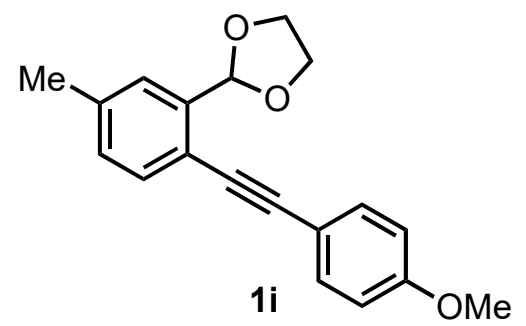

According to GP B, 2-((4-methoxyphenyl)ethynyl)-5-methylbenzaldehyde (S1i, $750.9 \mathrm{mg}, 3.0 \mathrm{mmol})$, ethylene glycol (1.7 $\mathrm{mL}, 30.0 \mathrm{mmol}), \mathrm{CH}(\mathrm{OMe})_{3}(492.0 \mu \mathrm{L}, 4.5 \mathrm{mmol})$, TBATB $(14.5 \mathrm{mg}, 30.0 \mu \mathrm{mol})$ and THF $(2 \mathrm{~mL})$ were stirred for $3 \mathrm{~h}$ at room temperature. Purification by flash silica-gel column chromatography (hexane / ethyl acetate $=15 / 1$ ) to give 2-(2-((4-methoxyphenyl)ethynyl)-5-methylphenyl)-1,3-dioxolane (1i, $415.0 \mathrm{mg}, 1.4 \mathrm{mmol})$ in 47\% yieldc as a pale yellow solid.

MP: $98-100{ }^{\circ} \mathrm{C} ;{ }^{1} \mathrm{H}$ NMR $\left(500 \mathrm{MHz}, \mathrm{CDCl}_{3}\right): \delta 7.48-7.45(\mathrm{~m}, 3 \mathrm{H}), 7.36(\mathrm{~s}, 1 \mathrm{H}), 7.16(\mathrm{~d}, J=8.0 \mathrm{~Hz}, 1 \mathrm{H}), 6.88(\mathrm{~d}, J=8.5$ $\mathrm{Hz}, 2 \mathrm{H}), 6.25(\mathrm{~s}, 1 \mathrm{H}), 4.22-4.15(\mathrm{~m}, 2 \mathrm{H}), 4.10-4.03(\mathrm{~m}, 2 \mathrm{H}), 3.83(\mathrm{~s}, 3 \mathrm{H}), 2.34$ (s, 3H); ${ }^{13} \mathrm{C}^{\mathrm{NMR}}\left(125 \mathrm{MHz}, \mathrm{CDCl}_{3}\right): \delta$ 159.6, 138.9, 135.8, 133.0, 132.8, 129.1, 126.1, 122.6, 115.4, 113.9, 102.1, 93.5, 85.5, 65.5, 55.3, 21.0; IR (ATR) $\mathrm{cm}^{-1}$ : 2882, 2838, 2209, 1661, 1566, 1510, 1469, 1439, 1391, 239+, 2359, 1188, 1169, 1153, 1110, 1071, 1023; ESI-HRMS m/z: $317.1145\left([\mathrm{M}+\mathrm{Na}]^{+}\right)$; Calcd. for $\mathrm{C}_{19} \mathrm{H}_{18} \mathrm{O}_{3} \mathrm{Na}$ : 317.1148 .

\section{5-Methoxy-2-((4-methoxyphenyl)ethynyl)benzaldehyde (S1j)}

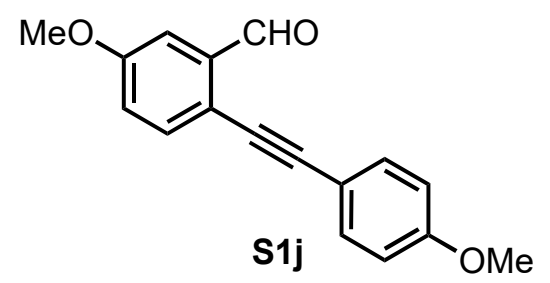

According to GP A, 2-bromo-5-methoxybenzaldehyde (1.1 g, $5.0 \mathrm{mmol})$, 4-ethynylanisole $(713.0 \mu \mathrm{L}, 5.5 \mathrm{mmol})$, $\mathrm{Pd}\left(\mathrm{PPh}_{3}\right)_{2} \mathrm{Cl}_{2}(175.5 \mathrm{mg}, 250.0 \mu \mathrm{mol}), \mathrm{CuI}(47.6 \mathrm{mg}, 250.0 \mu \mathrm{mol}), \mathrm{Et}_{3} \mathrm{~N}(4 \mathrm{~mL})$ and THF $(6 \mathrm{~mL})$ were stirred for $6 \mathrm{~h}$ at $50{ }^{\circ} \mathrm{C}$ in oil bath. Purification by flash silica-gel column chromatography (hexane / ethyl acetate $=15 / 1$ ) to give 5-methoxy-2-((4-methoxyphenyl)ethynyl)benzaldehyde (S1j, $877.0 \mathrm{mg}, 3.3 \mathrm{mmol})$ in $66 \%$ yield as a pale yellow solid. ${ }^{1} \mathrm{H}$ NMR (500 MHz, CDCl $): \delta 10.61(\mathrm{~s}, 1 \mathrm{H}), 7.54(\mathrm{~d}, J=10.8 \mathrm{~Hz}, 1 \mathrm{H}), 7.48(\mathrm{~d}, J=11.3 \mathrm{~Hz}, 1 \mathrm{H}), 7.42(\mathrm{~d}, J=3.5 \mathrm{~Hz}, 1 \mathrm{H})$, $7.14(\mathrm{dd}, J=3.5,10.8 \mathrm{~Hz}, 1 \mathrm{H}), 6.90(\mathrm{~d}, J=11.3 \mathrm{~Hz}, 2 \mathrm{H}), 3.88(\mathrm{~s}, 3 \mathrm{H}), 3.85(\mathrm{~s}, 3 \mathrm{H})$. Spectroscopic data of ${ }^{1} \mathrm{H}$ NMR was identical to that of the reference 1 .

2-(5-Methoxy-2-((4-methoxyphenyl)ethynyl)phenyl)-1,3-dioxolane (1j) 


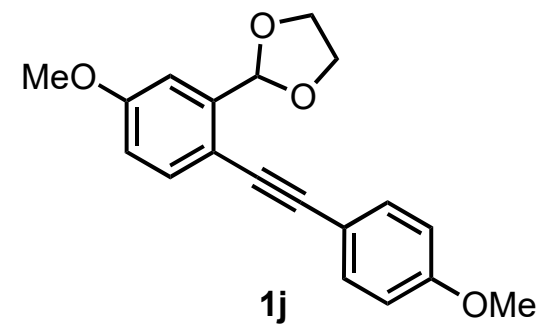

According to GP B, 5-methoxy-2-((4-methoxyphenyl)ethynyl)benzaldehyde (S1j, $798.9 \mathrm{mg}$, $3.0 \mathrm{mmol})$, ethylene glycol $(1.7 \mathrm{~mL}, 30.0 \mathrm{mmol}), \mathrm{CH}(\mathrm{OMe})_{3}(492.0 \mu \mathrm{L}, 4.5 \mathrm{mmol})$, TBATB $(14.5 \mathrm{mg}, 30.0 \mu \mathrm{mol})$ and THF (2 mL) were stirred for $3 \mathrm{~h}$ at room temperature. Purification by flash silica-gel column chromatography (hexane $/$ ethyl acetate $=10 / 1$ ) to give 2-(5-methoxy-2-((4-methoxyphenyl)ethynyl)phenyl)-1,3-dioxolane (1j, $642.4 \mathrm{mg}, 2.1 \mathrm{mmol})$ in 69\% yield as a pale yellow solid.

MP: $105-106{ }^{\circ} \mathrm{C} ;{ }^{1} \mathrm{H}$ NMR $\left(400 \mathrm{MHz}, \mathrm{CDCl}_{3}\right): \delta 7.47-7.43(\mathrm{~m}, 3 \mathrm{H}), 7.13(\mathrm{~d}, J=2.8 \mathrm{~Hz}, 1 \mathrm{H}), 6.89-6.86(\mathrm{~m}, 3 \mathrm{H}), 6.24(\mathrm{~s}$, 1H), 4.24-4.16 (m, 2H), 4.12-4.06 (m, 2H), $3.84(\mathrm{~s}, 3 \mathrm{H}), 3.83(\mathrm{~s}, 3 \mathrm{H}) ;{ }^{13} \mathrm{C} \mathrm{NMR}\left(100 \mathrm{MHz}, \mathrm{CDCl}_{3}\right): \delta 159.5,159.4,140.3$, 133.7, 132.9, 115.6, 115.2, 115.0, 113.9, 111.1, 101.9, 92.4, 85.2, 65.5, 55.4, 55.3; IR (ATR) $\mathrm{cm}^{-1}: 3066,2960,2890,2837$, 2758, 1609, 1567, 1510, 1459, 1433, 1392, 1307, 1291, 1246, 1224, 1198, 1166, 1109, 1064, 1024; ESI-HRMS m/z: $333.1099\left([\mathrm{M}+\mathrm{Na}]^{+}\right)$; Calcd. for $\mathrm{C}_{19} \mathrm{H}_{18} \mathrm{O}_{4} \mathrm{Na}: 333.1097$.

\section{2-Bromo-5-nitrobenzaldehyde (S1 k-1)}<smiles>O=Cc1cc([N+](=O)[O-])ccc1Br</smiles>

To a solution of 2-bromobenzaldehyde $(681.0 \mu \mathrm{L}, 1.6 \mathrm{mmol})$ and $\mathrm{HNO}_{3}(1 \mathrm{~mL})$ was added conc. $\mathrm{H}_{2} \mathrm{SO}_{4}(7.5 \mathrm{~mL})$ at $0{ }^{\circ} \mathrm{C}$ under argon. The reaction mixture was stirred at room temperature for $12 \mathrm{~h}$. To the reaction mixture was added $\mathrm{H}_{2} \mathrm{O}$ at $0{ }^{\circ} \mathrm{C}$ and extracted with ethyl acetate. The combined organic layers were dried over $\mathrm{Na}_{2} \mathrm{SO}_{4}$ and concentrated in vacuo. The residue was purified by silica-gel column chromatography (hexane / ethyl acetate = 4 / 1) to give 2-bromo-5-nitrobenzaldehyde (S1 k-1, $345.0 \mathrm{mg}, 1.5 \mathrm{mmol})$ in 95\% yield as a pale yellow solid.

${ }^{1} \mathrm{H}$ NMR $\left.500 \mathrm{MHz}, \mathrm{CDCl}_{3}\right): \delta 10.40(\mathrm{~s}, 1 \mathrm{H}), 8.73(\mathrm{~d}, J=3.5 \mathrm{~Hz}, 1 \mathrm{H}), 8.30(\mathrm{dd}, J=3.5,10.5 \mathrm{~Hz}, 1 \mathrm{H}), 7.89(\mathrm{~d}, J=10.5 \mathrm{~Hz}$, 1H). Spectroscopic data of ${ }^{1} \mathrm{H}$ NMR was identical to that of the reference 6 .

\section{2-((4-Methoxyphenyl)ethynyl)-5-nitrobenzaldehyde (S1 k-2)}

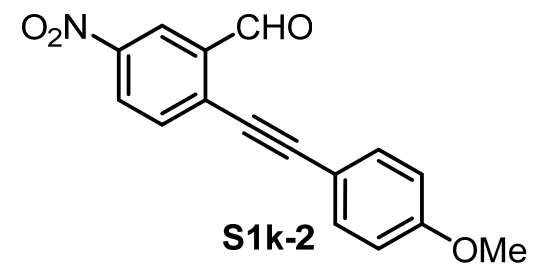

According to GP A, 2-bromo-5-nitrobenzaldehyde (S1k-1, $1.0 \mathrm{~g}, 4.5 \mathrm{mmol})$, 4-ethynylanisole (713.7 mg, $5.4 \mathrm{mmol})$, $\mathrm{Pd}\left(\mathrm{PPh}_{3}\right)_{2} \mathrm{Cl}_{2}(94.8 \mathrm{mg}, 135.0 \mu \mathrm{mol}), \mathrm{CuI}(25.7 \mathrm{mg}, 135.0 \mu \mathrm{mol}), \mathrm{Et}_{3} \mathrm{~N}(10 \mathrm{~mL})$ and $\mathrm{THF}(15 \mathrm{~mL})$ were stirred for $17 \mathrm{~h}$ at $50{ }^{\circ} \mathrm{C}$ in oil bath. Purification by flash silica-gel column chromatography (hexane / ethyl acetate $=10 / 1$ ) to give 2-((4-methoxyphenyl)ethynyl)-5-nitrobenzaldehyde (S1k-2, $361.0 \mathrm{mg}, 1.3 \mathrm{mmol})$ in 29\% yield as a yellow solid.

${ }^{1} \mathrm{H}$ NMR (400 MHz, $\left.\mathrm{CDCl}_{3}\right): \delta 10.65(\mathrm{~s}, 1 \mathrm{H}), 8.76(\mathrm{~d}, J=1.8 \mathrm{~Hz}, 1 \mathrm{H}), 8.41(\mathrm{dd}, J=1.8,6.8 \mathrm{~Hz}, 1 \mathrm{H}), 7.79(\mathrm{~d}, J=6.8 \mathrm{~Hz}$, 1H), $7.55(\mathrm{~d}, J=7.4 \mathrm{~Hz}, 2 \mathrm{H}), 6.95(\mathrm{~d}, J=7.4 \mathrm{~Hz}, 2 \mathrm{H}), 3.87(\mathrm{~s}, 3 \mathrm{H})$. Spectroscopic data of ${ }^{1} \mathrm{H}$ NMR was identical to that of the reference 7 . 


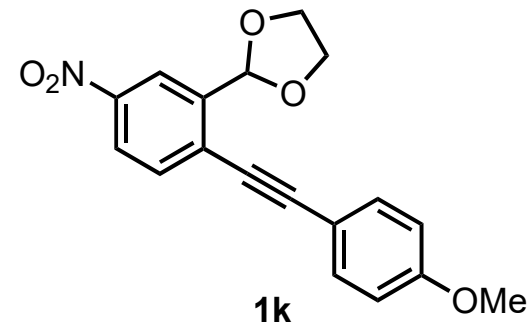

To a solution of 2-((4-methoxyphenyl)ethynyl)-5-nitrobenzaldehyde (S1k-2, $135.0 \mathrm{mg}, 480.0 \mu \mathrm{mol})$ and ethylene glycol $(268.0 \mu \mathrm{L}, 4.8 \mathrm{mmol})$ in toluene $(4 \mathrm{~mL})$ was added $p$ - $\mathrm{TsOH}(34.4 \mathrm{mg}, 200.0 \mu \mathrm{mol})$ at room temperature under argon. The reaction mixture was stirred at $130{ }^{\circ} \mathrm{C}$ in oil bath for $24 \mathrm{~h}$. To the reaction mixture was added $\mathrm{NaHCO}_{3}$ aq. at room temperature and extracted with ethyl acetate. The combined organic layers were dried over $\mathrm{Na}_{2} \mathrm{SO}_{4}$, and concentrated in vacuo. The residue was purified by silica-gel column chromatography (hexane / ethyl acetate $=5 / 1$ ) to give 2-(2-((4-methoxyphenyl)ethynyl)-5-nitrophenyl)-1,3-dioxolane (1k, $100.8 \mathrm{mg}, 310.0 \mu \mathrm{mol})$ in $65 \%$ yield as a yellow solid. MP: $100-102{ }^{\circ} \mathrm{C} ;{ }^{1} \mathrm{H}$ NMR $\left(400 \mathrm{MHz}, \mathrm{CDCl}_{3}\right): \delta 8.46(\mathrm{~d}, J=2.4 \mathrm{~Hz}, 1 \mathrm{H}), 8.20(\mathrm{dd}, J=2.4,8.0 \mathrm{~Hz}, 1 \mathrm{H}), 7.66(\mathrm{~d}, J=8.0$ $\mathrm{Hz}, 1 \mathrm{H}), 7.54(\mathrm{~d}, J=8.4,2 \mathrm{H}), 6.91(\mathrm{~d}, J=8.4 \mathrm{~Hz}, 2 \mathrm{H}), 6.30(\mathrm{~s}, 1 \mathrm{H}), 4.28-4.20(\mathrm{~m}, 2 \mathrm{H}), 4.17-4.09(\mathrm{~m}, 2 \mathrm{H}), 3.85(\mathrm{~s}, 3 \mathrm{H})$; ${ }^{13} \mathrm{C}$ NMR $\left(100 \mathrm{MHz}, \mathrm{CDCl}_{3}\right): \delta 160.5,146.8,140.6,133.5,133.0,129.6,123.8,121.7,114.8,114.1,100.9,99.7,84.1,65.8$, 55.4; IR (ATR) $\mathrm{cm}^{-1}:$ 3090, 2976, 2893, 2214, 1582, 1512, 1475, 1393, 1337, 1292, 1248, 1174, 1143, 1098, 1059, 1015; Anal. Calcd. for $\mathrm{C}_{18} \mathrm{H}_{15} \mathrm{NO}_{5}$ : C, 66.46; H, 4.65; N, 4.31. Found: C, 66.31; H, 4.65; N, 4.28.

\section{2-((Trimethylsilyl)ethynyl)benzaldehyde (S1l-1)}

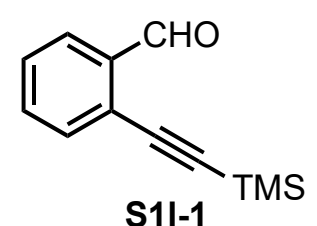

According to GP A, 2-bromobenzaldehyde $(1.1 \mathrm{~mL}, 15.0 \mathrm{mmol})$, trimethylsilylacetylene $(3.2 \mathrm{~mL}, 22.5 \mathrm{mmol})$, $\mathrm{Pd}\left(\mathrm{PPh}_{3}\right)_{2} \mathrm{Cl}_{2}(526.0 \mathrm{mg}, 750.0 \mu \mathrm{mol}), \mathrm{CuI}(142.8 \mathrm{mg}, 750.0 \mu \mathrm{mol}), \mathrm{Et}_{3} \mathrm{~N}(6 \mathrm{~mL})$ and THF $(50 \mathrm{~mL})$ were stirred for $24 \mathrm{~h}$ at room temperature. Purification by flash silica-gel column chromatography (hexane / ethyl acetate $=40 / 1$ ) to give 2-((trimethylsilyl)ethynyl)benzaldehyde (S1l-1, $2.1 \mathrm{~g}, 10.5 \mathrm{mmol})$ in 70\% yield as a pale yellow oil.

${ }^{1} \mathrm{H}$ NMR (400 MHz, CDCl $): \delta 10.56(\mathrm{~s}, 1 \mathrm{H}), 7.91(\mathrm{~d}, J=8.0 \mathrm{~Hz}, 1 \mathrm{H}), 7.59-7.52(\mathrm{~m}, 2 \mathrm{H}), 7.44(\mathrm{t}, J=8.0 \mathrm{~Hz}, 1 \mathrm{H}), 0.28(\mathrm{~s}$, 9H) Spectroscopic data of ${ }^{1} \mathrm{H}$ NMR was identical to that of the reference 8 .

2-Ethynylbenzaldehyde (S1I-2)<smiles>C#Cc1ccccc1C=O</smiles>

S1I-2

To a solution of 2-((trimethylsilyl)ethynyl)benzaldehyde (S1l-1, $384.2 \mathrm{mg}, 1.6 \mathrm{mmol})$ in $\mathrm{MeOH}(10 \mathrm{~mL})$ was added $\mathrm{K}_{2} \mathrm{CO}_{3}$ (258.7 mg, $1.9 \mathrm{mmol}$ ) at room temperature under argon and stirred for $1 \mathrm{~h}$. The reaction mixture was quenched with $\mathrm{H}_{2} \mathrm{O}$ and extracted with ethyl acetate. The combined organic layers were dried over $\mathrm{Na}_{2} \mathrm{SO}_{4}$ and concentrated in vacuo. The residue was purified by silica-gel column chromatography (hexane / ethyl acetate $=10 / 1$ ) to give 2-ethynylbenzaldehyde (S1l-2, $271.8 \mathrm{mg}, 1.6 \mathrm{mmol})$ in quantitative yield as a pale yellow oil.

${ }^{1} \mathrm{H}$ NMR (400 MHz, $\left.\mathrm{CDCl}_{3}\right): \delta 10.55(\mathrm{~s}, 1 \mathrm{H}), 7.94(\mathrm{~d}, J=7.6 \mathrm{~Hz}, 1 \mathrm{H}), 7.64-7.56$ (m, 2H), 7.49 (dd, $\left.J=7.6,7.6 \mathrm{~Hz}, 1 \mathrm{H}\right)$, 
3.47 (s, 1H). Spectroscopic data of ${ }^{1} \mathrm{H}$ NMR was identical to that of the reference 9.

2-((4-Acetylphenyl)ethynyl)benzaldehyde (S11-3)

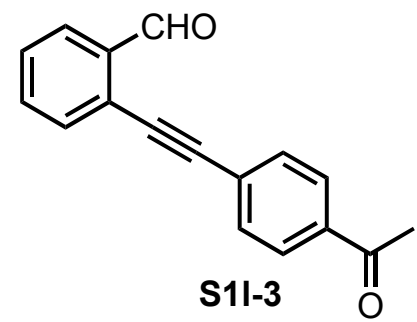

According to GP A, 4'-iodoacetophenone (738.2 mg, $3.0 \mathrm{mmol}$ ), 2-ethynylbenzaldehyde (S1l-2, $260.3 \mathrm{mg}, 2.0 \mathrm{mmol}$ ), $\mathrm{Pd}\left(\mathrm{PPh}_{3}\right)_{2} \mathrm{Cl}_{2}(28.1 \mathrm{mg}, 40.0 \mu \mathrm{mol}), \mathrm{CuI}(15.2 \mathrm{mg}, 80.0 \mu \mathrm{mol}), \mathrm{Et}_{3} \mathrm{~N}(5 \mathrm{~mL})$ and THF $(5 \mathrm{~mL})$ were stirred for $24 \mathrm{~h}$ at room temperature. Purification by flash silica-gel column chromatography (hexane / ethyl acetate $=5 / 1$ ) to give

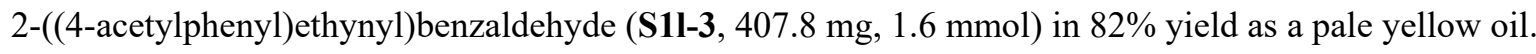

${ }^{1} \mathrm{H}$ NMR (400 MHz, $\mathrm{CDCl}_{3}$ ): $\delta 10.64$ (s, 1H), 7.99-7.97 (m, 3H), 7.69-7.61 (m, 4H), 7.51 (dd, J = 7.6, 7.6 Hz, 1H), 2.64 (s, $3 \mathrm{H})$. Spectroscopic data of ${ }^{1} \mathrm{H}$ NMR was identical to that of the reference 10.

2-(4-((2-(1,3-Dioxolan-2-yl)phenyl)ethynyl)phenyl)-2-methyl-1,3-dioxolane (11)

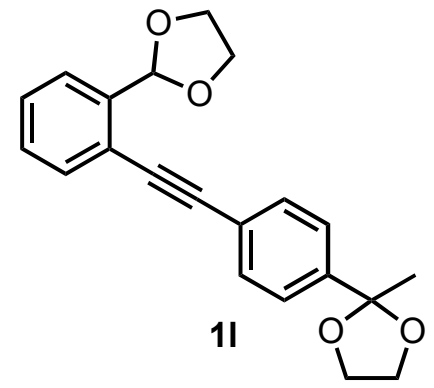

According to GP B, 2-((4-acetylphenyl)ethynyl)benzaldehyde (S11-3, $406.0 \mathrm{mg}, 1.2 \mathrm{mmol})$, ethylene glycol (530.0 $\mu \mathrm{L}, 9.6$ $\mathrm{mmol}), \mathrm{CH}(\mathrm{OMe})_{3}(570.0 \mu \mathrm{L}, 5.3 \mathrm{mmol})$, TBATB $(23.0 \mathrm{mg}, 48.0 \mu \mathrm{mol})$ and THF $(2 \mathrm{~mL})$ were stirred for $3 \mathrm{~h}$ at room temperature. Purification by flash silica-gel column chromatograhy (hexane / ethyl acetate $=5 / 1$ ) to give 2-(4-((2-(1,3-dioxolan-2-yl)phenyl)ethynyl)phenyl)-2-methyl-1,3-dioxolane (11, p131.4 mg, $400.0 \mu \mathrm{mol})$ in 33\% yield as a colorless solid.

MP: $118-119{ }^{\circ} \mathrm{C} ;{ }^{1} \mathrm{H}$ NMR (400 MHz, $\left.\mathrm{CDCl}_{3}\right): \delta$ 7.61-7.59 (m, 1H), 7.56-7.54 (m, 1H), 7.53-7.46 (m, 4H), 7.40-7.32 (m, $2 \mathrm{H}), 6.28(\mathrm{~s}, 1 \mathrm{H}), 4.24-4.16(\mathrm{~m}, 2 \mathrm{H}), 4.12-4.00(\mathrm{~m}, 4 \mathrm{H}), 3.82-3.73(\mathrm{~m}, 2 \mathrm{H}), 1.66(\mathrm{~s}, 3 \mathrm{H}) ;{ }^{13} \mathrm{C} \mathrm{NMR}\left(100 \mathrm{MHz}, \mathrm{CDCl}_{3}\right): \delta$ 143.6, 138.9, 132.5, 131.5, 129.0, 128.5, 126.2, 125.3, 122.7, 122.4, 108.6, 102.1, 93.7, 86.7, 65.8, 64.5, 27.4; IR (ATR) $\mathrm{cm}^{-1}: 2986,2885,1606,1572,1509,1475,1448,1396,1373,1247,1198,1140,1109,1094,1069,1033$; ESI-HRMS m/z: $337.1439\left([\mathrm{M}+\mathrm{H}]^{+}\right)$; Calcd. for $\mathrm{C}_{21} \mathrm{H}_{21} \mathrm{O}_{4}: 337.1434$.

\section{2-(Cyclohex-1-en-1-ylethynyl)benzaldehyde (S1 m)}

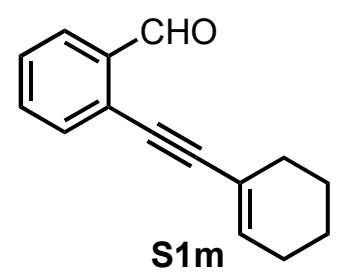

According to GP A, 2-bromobenzaldehyde (740.1 mg, $4.0 \mathrm{mmol})$, 1-ethynylcyclohexene (543.0 $\mu \mathrm{L}, 4.4 \mathrm{mmol})$, $\mathrm{Pd}\left(\mathrm{PPh}_{3}\right)_{2} \mathrm{Cl}_{2}(84.2 \mathrm{mg}, 120.0 \mu \mathrm{mol}), \mathrm{CuI}(22.9 \mathrm{mg}, 120.0 \mu \mathrm{mol}), \mathrm{Et}_{3} \mathrm{~N}(5 \mathrm{~mL})$ and THF $(8 \mathrm{~mL})$ were stirred for $2 \mathrm{~h}$ at $80{ }^{\circ} \mathrm{C}$ in oil bath. Purification by flash silica-gel column chromatography (hexane / ethyl acetate $=20 / 1$ ) to give 
2-(cyclohex-1-en-1-ylethynyl)benzaldehyde ( $\mathbf{S 1 m}, 845.6 \mathrm{mg}, 4.0 \mathrm{mmol})$ in 99\% yield as a pale yellow oil.

${ }^{1} \mathrm{H}$ NMR $\left(400 \mathrm{MHz}, \mathrm{CDCl}_{3}\right): \delta 10.55(\mathrm{~s}, 1 \mathrm{H}), 7.90(\mathrm{~d}, J=8.0 \mathrm{~Hz}, 1 \mathrm{H}), 7.54-7.51(\mathrm{~m}, 2 \mathrm{H}), 7.41-7.37(\mathrm{~m}, 1 \mathrm{H}), 6.32-6.29(\mathrm{~m}$, $1 \mathrm{H}), 2.27-2.23(\mathrm{~m}, 2 \mathrm{H}), 2.20-2.16(\mathrm{~m}, 2 \mathrm{H}), 1.74-1.61(\mathrm{~m}, 4 \mathrm{H})$. Spectroscopic data of ${ }^{1} \mathrm{H}$ NMR was identical to that of the reference 11 .

2-(2-(Cyclohex-1-enethynyl)phenyl-1,3-dioxolane (1m)<smiles>C(#Cc1ccccc1C1OCCO1)C1=CCCCC1</smiles>

According to GP B, 2-(cyclohex-1-en-1-ylethynyl)benzaldehyde (S1m, $630.8 \mathrm{mg}, 3.0 \mathrm{mmol}$ ), ethylene glycol (3.3 mL, 30.0 $\mathrm{mmol}), \mathrm{CH}(\mathrm{OMe})_{3}(492.0 \mu \mathrm{L}, 4.5 \mathrm{mmol})$, TBATB $(14.4 \mathrm{mg}, 30.0 \mu \mathrm{mol})$ and THF $(3 \mathrm{~mL})$ were stirred for $12 \mathrm{~h}$ at room temperature. Purification by flash silica-gel column chromatography (hexane / ethyl acetate $=15 / 1$ ) to give 2-(2-(cyclohex-1-enethynyl)phenyl-1,3-dioxolane (1 m, $406.7 \mathrm{mg}, 1.6 \mathrm{mmol})$ in 53\% yield as a pale yellow oil.

${ }^{1} \mathrm{H}$ NMR (400 MHz, $\mathrm{CDCl}_{3}$ ): $\delta$ 7.56-7.54 (m, 1H), 7.46-7.41 (m, 1H), 7.32-7.29 (m, 2H), 6.24-6.20 (m, 2H), 4.22-4.13 (m, $2 \mathrm{H}), 4.10-4.02(\mathrm{~m}, 2 \mathrm{H}), 2.26-2.21(\mathrm{~m}, 2 \mathrm{H}), 2.18-2.12(\mathrm{~m}, 2 \mathrm{H}), 1.71-1.58(\mathrm{~m}, 4 \mathrm{H}) ;{ }^{13} \mathrm{C} \mathrm{NMR}\left(100 \mathrm{MHz}, \mathrm{CDCl}_{3}\right): \delta 138.5$, 135.4, 132.3, 128.9, 127.9, 126.0, 123.1, 120.1, 102.0, 95.9, 83.9, 65.5, 29.1, 25.7, 22.3, 21.5; IR (ATR) $\mathrm{cm}^{-1}: 2928 \mathrm{~m} 2833$, 2201, 1599, 2571, 1482, 1448, 1392, 1347, 1273, 1199, 1123, 1098, 1067, 1027; ESI-HRMS m/z: 277.1203 ([M+Na] $\left.{ }^{+}\right)$; Calcd. for $\mathrm{C}_{17} \mathrm{H}_{18} \mathrm{O}_{2} \mathrm{Na}$ : 277.1199.

\section{2-(Oct-1-yn-1-yl)benzaldehyde (S1n)}<smiles>CCC#Cc1ccccc1C=O</smiles>

According to GP A, 2-bromobenzaldehyde (925.1 mg, $5.0 \mathrm{mmol}), 1$-octhyne (1.1 mL, $7.5 \mathrm{mmol}), \mathrm{Pd}_{(}\left(\mathrm{PPh}_{3}\right)_{2} \mathrm{Cl}_{2}(675.5 \mathrm{mg}$, $250.0 \mu \mathrm{mol}), \mathrm{CuI}(47.6 \mathrm{mg}, 250.0 \mu \mathrm{mol}), \mathrm{Et}_{3} \mathrm{~N}(5 \mathrm{~mL})$ and THF $(8 \mathrm{~mL})$ were stirred for $2 \mathrm{~h}$ at $80{ }^{\circ} \mathrm{C}$ in oil bath. Purification by flash silica-gel column chromatography (hexane / ethyl acetate $=40 / 1)$ to give 2-(oct-1-yn-1-yl)benzaldehyde (S1n, 1.1 $\mathrm{g}, 4.9 \mathrm{mmol})$ in $98 \%$ yield as a brown oil.

${ }^{1} \mathrm{H}$ NMR $\left(400 \mathrm{MHz}, \mathrm{CDCl}_{3}\right): \delta 10.54(\mathrm{~s}, 1 \mathrm{H}), 7.89(\mathrm{~d}, J=7.6 \mathrm{~Hz}, 1 \mathrm{H}), 7.54-7.5(\mathrm{~m}, 2 \mathrm{H}), 7.40-7.37(\mathrm{~m}, 1 \mathrm{H}), 2.48(\mathrm{t}, J=7.2$ $\mathrm{Hz}, 2 \mathrm{H}), 1.68-1.60(\mathrm{~m}, 2 \mathrm{H}), 1.50-1.45(\mathrm{~m}, 2 \mathrm{H}), 1.35-1.33(\mathrm{~m}, 2 \mathrm{H}), 0.91(\mathrm{t}, J=5.8 \mathrm{~Hz}, 3 \mathrm{H})$. Spectroscopic data of ${ }^{1} \mathrm{H}$ NMR was identical to that of the reference 12 .

\section{2-(2-Oct-1-yn-1lethynyl)phenyl)-1,3-dioxolane (1n)}

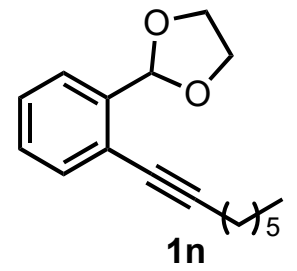

According to GP B, 2-(oct-1-yn-1-yl)benzaldehyde (S1n, $642.9 \mathrm{mg}, 3.0 \mathrm{mmol})$, ethylene glycol (3.3 mL, $30.0 \mathrm{mmol})$, $\mathrm{CH}(\mathrm{OMe})_{3}(495.0 \mu \mathrm{L}, 4.5 \mathrm{mmol})$, TBATB $(14.5 \mathrm{mg}, 30.0 \mu \mathrm{mol})$ and THF $(3 \mathrm{~mL})$ were stirred for $3 \mathrm{~h}$ at room temperature. 
Purification by flash silica-gel column chromatography (hexane / ethyl acetate = 20 / 1) to give 2-(2-oct-1-yn-1 lethynyl)phenyl)-1,3-dioxolane (1n, $471.7 \mathrm{mg}, 1.8 \mathrm{mmol})$ in $61 \%$ yield as a pale yellow oil.

${ }^{1} \mathrm{H}$ NMR (400 MHz, $\left.\mathrm{CDCl}_{3}\right): \delta$ 7.57-7.53 (m, 1H), 7.43-7.40 (m, 1H), 7.32-7.27 (m, 2H), 6.21 (s, 1H), 4.21-4.13 (m, 2H), $4.11-4.01(\mathrm{~m}, 2 \mathrm{H}), 2.45(\mathrm{t}, J=7.0 \mathrm{~Hz}, 2 \mathrm{H}), 1.65-1.58(\mathrm{~m}, 2 \mathrm{H}), 1.51-1.43(\mathrm{~m}, 2 \mathrm{H}), 1.36-1.27(\mathrm{~m}, 4 \mathrm{H}), 0.91(\mathrm{t}, J=7.2 \mathrm{~Hz}$, $3 \mathrm{H}) ;{ }^{13} \mathrm{C}$ NMR $\left(100 \mathrm{MHz}, \mathrm{CDCl}_{3}\right): \delta$ 138.6, 132.5, 128.9, 127.6, 125.8, 123.4, 102.0, 95.3, 77.7, 65.5, 31.4, 28.6, 28.6, 22.6, 19.6, 14.1; IR (ATR) $\mathrm{cm}^{-1}:$ 2928, 2858, 2226, 1602, 1573, 1451, 1393, 1249, 1198, 1115, 1072, 1029; ESI-HRMS m/z: $281.1503\left([\mathrm{M}+\mathrm{Na}]^{+}\right)$; Calcd. for $\mathrm{C}_{17} \mathrm{H}_{22} \mathrm{O}_{2} \mathrm{Na}: 281.1512$.

\section{2-(4-Hydroxy-1-butyn-1-yl)bazaldehyde (S10-1)}<smiles>O=Cc1ccccc1C#CC(O)O</smiles>

According to GP A, 2-bromobenzaldehyde (910.0 mg, $5.0 \mathrm{mmol})$, 3-butyn-1-ol $\left.(346.0 \mu \mathrm{L}, 6.0 \mathrm{mmol}), \mathrm{Pd}_{(\mathrm{PPh}}\right)_{2} \mathrm{Cl}_{2}(105.3$ $\mathrm{mg}, 150.0 \mu \mathrm{mol}), \mathrm{CuI}(28.6 \mathrm{mg}, 150.0 \mu \mathrm{mol}), \mathrm{Et}_{3} \mathrm{~N}(3 \mathrm{~mL})$ and $\mathrm{THF}(6 \mathrm{~mL})$ were stirred for $6 \mathrm{~h}$ at room temperature. Purification by flash silica-gel column chromatography (hexane / ethyl acetate = 2 / 1) to give 2-(4-hydroxy-1-butyn-1-yl)benzaldehyde (S1o-1, $535.1 \mathrm{mg}, 3.1 \mathrm{mmol})$ in 62\% yield as a pale yellow oil.

${ }^{1} \mathrm{H}$ NMR $\left(500 \mathrm{MHz}, \mathrm{CDCl}_{3}\right): \delta 10.45(\mathrm{~s}, 1 \mathrm{H}), 7.87(\mathrm{~d}, J=8.0 \mathrm{~Hz}, 1 \mathrm{H}), 7.56-7.51(\mathrm{~m}, 2 \mathrm{H}), 7.45-7.41(\mathrm{~m}, 1 \mathrm{H}), 3.88(\mathrm{dt}, J=$ 6.0, $6.5 \mathrm{~Hz}, 2 \mathrm{H}), 2.77(\mathrm{t}, J=6.5 \mathrm{~Hz}, 2 \mathrm{H}), 2.27-2.25(\mathrm{~m}, 1 \mathrm{H})$. Spectroscopic data of ${ }^{1} \mathrm{H}$ NMR was identical to that of the reference 13 .

\section{4-(2-(1,3-Dioxolan-2-yl)phenyl)but-3-yn-1-ol (S1o-2)}<smiles>OC(O)C#Cc1ccccc1C1OCCO1</smiles>

According to GP B, 2-(4-hydroxy-1-butyn-1-yl)benzaldehyde (S1o-1, 1.5 g, 8.7 mmol), ethylene glycol (10 mL, 173.2 mmol $), \mathrm{CH}(\mathrm{OMe})_{3}(1.4 \mathrm{~mL}, 12.9 \mathrm{mmol})$, TBATB $(41.8 \mathrm{mg}, 870.0 \mu \mathrm{mol})$ and $\mathrm{THF}(5 \mathrm{~mL})$ were stirred for $20 \mathrm{~h}$ at room temperature. Purification by flash silica-gel column chromatography (hexane / ethyl acetate = $3 / 1$ ) to give 4-(2-(1,3-dioxolan-2-yl)phenyl)but-3-yn-1-ol (S1o-2, $905.8 \mathrm{mg}, 5.2 \mathrm{mmol})$ in 60\% yield as a pale yellow oil.

${ }^{1} \mathrm{H}$ NMR (400 MHz, $\left.\mathrm{CDCl}_{3}\right): \delta 7.57(\mathrm{~d}, J=6.8 \mathrm{~Hz}, 1 \mathrm{H}), 7.44-7.42(\mathrm{~m}, 1 \mathrm{H}), 7.35-7.28(\mathrm{~m}, 2 \mathrm{H}), 6.23(\mathrm{~s}, 1 \mathrm{H}), 4.17-4.04(\mathrm{~m}$, $4 \mathrm{H}), 3.83(\mathrm{t}, J=6.0 \mathrm{~Hz}, 2 \mathrm{H}), 2.84(\mathrm{brs}, 1 \mathrm{H}), 2.71(\mathrm{t}, J=6.0 \mathrm{~Hz}, 2 \mathrm{H})$. Spectroscopic data of ${ }^{1} \mathrm{H}$ NMR was identical to that of the reference 14 .

2-(2-(4-Methoxybut-1-yn-1-yl)phenyl)-1,3-dioxolane (10)<smiles>COCC#Cc1ccccc1C1OCCO1</smiles>

10 
To a solution of 4-(2-(1,3-dioxolan-2-yl)phenyl)but-3-yn-1-ol (S1o-2, $436.5 \mathrm{~mL}, 2.0 \mathrm{mmol})$ in THF (5 mL) was added NaH (60\% oil dispersion, $240 \mathrm{mg}, 6.0 \mathrm{mmol}$ ) at $0{ }^{\circ} \mathrm{C}$ under argon and stirred for $30 \mathrm{~min}$. To the reaction mixture was added MeI $\left(374.0 \mu \mathrm{L}, 6.0 \mathrm{mmol}\right.$ ) and stirred at room temperature for $3 \mathrm{~h}$. The reaction mixture was quenched with $\mathrm{H}_{2} \mathrm{O}$ at $0{ }^{\circ} \mathrm{C}$ and extracted with ethyl acetate. The combined organic layers were dried over $\mathrm{Na}_{2} \mathrm{SO}_{4}$, and concentrated in vacuo. The residue was purified by silica-gel column chromatography (hexane / ethyl acetate $=10 / 1$ ) to give 2-(2-(4-methoxybut-1-yn-1-yl)phenyl)-1,3-dioxolane (10, $325.2 \mathrm{mg}, 1.4 \mathrm{mmol}) \mathrm{in} 69 \%$ yield as a yellow oil.

${ }^{1} \mathrm{H}$ NMR (400 MHz, $\left.\mathrm{CDCl}_{3}\right): \delta 7.54(\mathrm{~d}, J=7.2 \mathrm{~Hz}, 1 \mathrm{H}), 7.43(\mathrm{~d}, J=7.2 \mathrm{~Hz}, 1 \mathrm{H}), 7.34-7.28(\mathrm{~m}, 2 \mathrm{H}), 6.20(\mathrm{~s}, 1 \mathrm{H})$, $4.21-4.13(\mathrm{~m}, 2 \mathrm{H}), 4.10-4.02(\mathrm{~m}, 2 \mathrm{H}), 3.61(\mathrm{t}, J=6.9 \mathrm{~Hz}, 2 \mathrm{H}), 3.41(\mathrm{~s}, 3 \mathrm{H}), 2.73$ (t, $J=6.9 \mathrm{~Hz}, 2 \mathrm{H}) ;{ }^{13} \mathrm{C} \mathrm{NMR}(125 \mathrm{MHz}$, $\mathrm{CDCl}_{3}$ ): $\delta 138.8,132.6,128.9,127.9,125.9,122.9,101.9,91.5,78.6,70.8,65.5,58.7,20.8$; IR (ATR) $\mathrm{cm}^{-1}: 2886,1725$, 1601, 1572, 1483, 1450, 1387, 1334, 1288, 1249, 1227, 1196, 1113, 1070, 1042, 1024; ESI-HRMS m/z: 255.1009 $\left([\mathrm{M}+\mathrm{Na}]^{+}\right)$; Calcd. for $\mathrm{C}_{14} \mathrm{H}_{16} \mathrm{O}_{3} \mathrm{Na}: 255.0992$.

\section{1-Bromo-2-folmyl-1-cyclohexene (S1p-1)}<smiles>O=CC1=C(Br)CCCC1</smiles>

To a solution of cyclohexanone $(3.0 \mathrm{~mL}, 30.0 \mathrm{mmol})$ in $\mathrm{CHCl}_{3}(40 \mathrm{~mL})$ was added $\mathrm{PBr}_{3}(7.7 \mathrm{~mL}, 81.0 \mathrm{mmol})$ at $0{ }^{\circ} \mathrm{C}$ under argon and stirred for $1 \mathrm{~h}$. To the reaction mixture was added DMF $(7.0 \mathrm{~mL}, 90.0 \mathrm{mmol})$ at $0{ }^{\circ} \mathrm{C}$, warm up to room temperature and stirred for $8 \mathrm{~h}$. The reaction mixture was quenched with saturated $\mathrm{NaHCO}_{3}$ aqueous solution and extracted with ethyl acetate. The combined organic layers were dried over $\mathrm{Na}_{2} \mathrm{SO}_{4}$ and concentrated in vacuo. The residue was purified by silica-gel column chromatography (hexane / ethyl acetate $=10 / 1$ ) to give 1-bromo-2-folmyl-1-cyclohexene (S1p-1, 4.0g g, $21.0 \mathrm{mmol}$ ) in 70\% yield as a pale yellow oil.

${ }^{1} \mathrm{H}$ NMR (400 MHz, $\mathrm{CDCl}_{3}$ ): $\delta 10.03(\mathrm{~s}, 1 \mathrm{H}), 2.77-2.73(\mathrm{~m}, 2 \mathrm{H}), 2.30-2.26(\mathrm{~m}, 2 \mathrm{H}), 1.80-1.66(\mathrm{~m}, 4 \mathrm{H})$. Spectroscopic data of ${ }^{1} \mathrm{H}$ NMR was identical to that of the reference 15 .

\section{2-((4-Methoxyphenyl)ethynyl)cyclohex-1-enecarbaldehyde (S1p-2)}

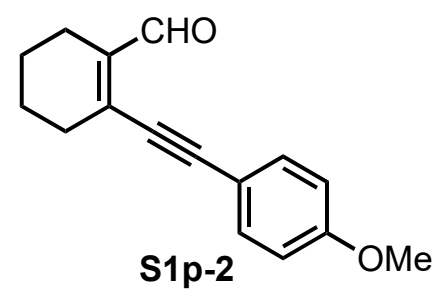

According to GP A, 1-bromo-2-folmyl-1-cyclohexene (S1p-1, $756.2 \mathrm{mg}, 4.0 \mathrm{mmol})$, 4-ethynylanisole (620.0 $\mu \mathrm{L}, 4.8 \mathrm{mmol})$, $\mathrm{Pd}\left(\mathrm{PPh}_{3}\right)_{2} \mathrm{Cl}_{2}(84.2 \mathrm{mg}, 120.0 \mu \mathrm{mol}), \mathrm{CuI}(22.9 \mathrm{mg}, 120.0 \mu \mathrm{mol}), \mathrm{Et}_{3} \mathrm{~N}(6 \mathrm{~mL})$ and THF $(9 \mathrm{~mL})$ were stirred for $24 \mathrm{~h}$ at room temperature. Purification by flash silica-gel column chromatography (hexane / ethyl acetate $=10 / 1$ ) to give 2-((4-methoxyphenyl)ethynyl)cyclohex-1-enecarbaldehyde (S1p-2, $517.3 \mathrm{mg}, 2.2 \mathrm{mmol})$ in 54\% yield as a pale yellow oil.

${ }^{1} \mathrm{H}$ NMR $\left(400 \mathrm{MHz}, \mathrm{CDCl}_{3}\right): \delta 10.31(\mathrm{~s}, 1 \mathrm{H}), 7.41(\mathrm{~d}, J=8.8 \mathrm{~Hz}, 2 \mathrm{H}), 6.88(\mathrm{~d}, J=8.8 \mathrm{~Hz}, 2 \mathrm{H}), 3.81(\mathrm{~s}, 3 \mathrm{H}), 2.52-2.48(\mathrm{~m}$, 2H), 2.32-2.28 (m, 2H), 1.74-1.64 (m, 4H). Spectroscopic data of ${ }^{1} \mathrm{H}$ NMR was identical to that of the reference 16.

\section{2-(2-((4-Methoxyphenyl)ethynyl)cyclohex-1-ene-1-yl)-1,3-dioxolane (1p)}




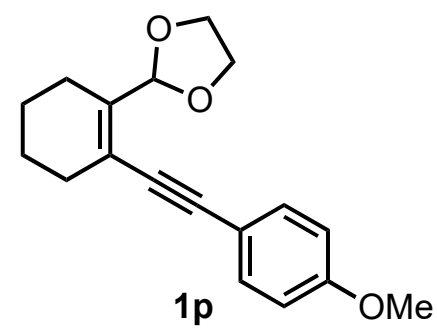

According to GP B, 2-((4-methoxyphenyl)ethynyl)cyclohex-1-enecarbaldehyde (S1p-2, $384.5 \mathrm{mg}, 1.6 \mathrm{mmol})$, ethylene glycol $(1.8 \mathrm{~mL}, 32.0 \mathrm{mmol}), \mathrm{CH}(\mathrm{OMe})_{3}(260.0 \mu \mathrm{L}, 2.4 \mathrm{mmol})$, TBATB $(23.1 \mathrm{mg}, 48.0 \mu \mathrm{mol})$ and THF (7.5 mL) were stirred for $24 \mathrm{~h}$ at room temperature. Purification by flash silica-gel column chromatography (hexane / ethyl acetate $=15 / 1)$ to give of 2-(2-((4-methoxyphenyl)ethynyl)cyclohex-1-ene-1-yl)-1,3-dioxolane (1p, $190.7 \mathrm{mg}, 671.0 \mu \mathrm{mol})$ in $42 \%$ yield as a pale yellow solid.

MP: $105-106{ }^{\circ} \mathrm{C} ;{ }^{1} \mathrm{H}$ NMR $\left(500 \mathrm{MHz}, \mathrm{CDCl}_{3}\right): \delta 7.36(\mathrm{~d}, J=8.5 \mathrm{~Hz}, 2 \mathrm{H}), 6.83(\mathrm{~d}, J=8.5 \mathrm{~Hz}, 2 \mathrm{H}), 5.97(\mathrm{~s}, 1 \mathrm{H}), 4.08-4.00$ $(\mathrm{m}, 2 \mathrm{H}), 3.98-3.92(\mathrm{~m}, 2 \mathrm{H}), 3.85(\mathrm{~S}, 3 \mathrm{H}), 2.30-2.28(\mathrm{~m}, 2 \mathrm{H}), 2.18-2.16(\mathrm{~m}, 2 \mathrm{H}), 1.67-1.64(\mathrm{~m}, 4 \mathrm{H}) ;{ }^{13} \mathrm{C} \mathrm{NMR}(100 \mathrm{MHz}$, $\left.\mathrm{CDCl}_{3}\right): \delta 159.4,139.7,132.8,122.3,115.7,113.8,103.2,93.1,86.4,65.6,55.3,30.7,22.2,21.9,21.7 ; \mathrm{IR}(\mathrm{ATR}) \mathrm{cm}^{-1}$ : 3001, 2959, 2929, 2880, 2835, 2199, 1601, 1506, 1463, 1442, 1296, 1245, 1218, 1185, 1174, 1125, 1109, 1088, 1065, 1053, 1029; ESI-HRMS m/z: $307.1305\left([\mathrm{M}+\mathrm{Na}]^{+}\right)$; Calcd. for $\mathrm{C}_{18} \mathrm{H}_{20} \mathrm{O}_{3} \mathrm{Na}: 307.1305$.

2-(2-((4-Methoxyphenyl)ethynyl)phenyl)-1,3,dioxane (1q)

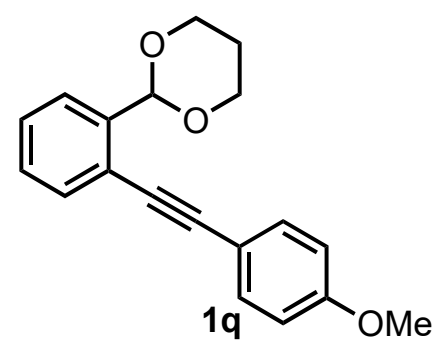

To a solution of 2-((4-methoxyphenyl)ethynyl)-benzaldehyde (S1a, $472.5 \mathrm{mg}, 2.0 \mathrm{mmol})$ and 1,3-propanediol (1.4 mL, 7.0 $\mathrm{mmol})$ in toluene $(10 \mathrm{~mL})$ was added $p-\mathrm{TsOH}(137.8 \mathrm{mg}, 0.8 \mathrm{mmol})$ at room temperature under argon. The reaction mixture was stirred at $130{ }^{\circ} \mathrm{C}$ in oil bath for $24 \mathrm{~h}$. To the reaction mixture was added $\mathrm{NaHCO}_{3}$ aq. at room temperature and extracted with ethyl acetate. The combined organic layers were dried over $\mathrm{Na}_{2} \mathrm{SO}_{4}$, and concentrated in vacuo. The residue was purified by silica-gel column chromatography (hexane / ethyl acetate = 30 / 1) to give 2-(2-((4-methoxyphenyl)ethynyl)phenyl)-1,3,dioxane (1q, $523.9 \mathrm{mg}, 1.8 \mathrm{mmol})$ in 89\% yield as a colorless solid.

MP: $44-45{ }^{\circ} \mathrm{C} ;{ }^{1} \mathrm{H}$ NMR (400 MHz, $\left.\mathrm{CDCl}_{3}\right): \delta 7.69(\mathrm{dd}, J=1.6,7.2 \mathrm{~Hz}, 1 \mathrm{H}), 7.52-7.47(\mathrm{~m}, 3 \mathrm{H}), 7.38-7.29(\mathrm{~m}, 2 \mathrm{H}), 6.90$ $(\mathrm{d}, J=8.8 \mathrm{~Hz}, 2 \mathrm{H}), 5.98(\mathrm{~s}, 1 \mathrm{H}), 4.32-4.28(\mathrm{~m}, 2 \mathrm{H}), 4.05(\mathrm{ddd}, J=2.4,12.4,12.4 \mathrm{~Hz}, 2 \mathrm{H}), 3.84(\mathrm{~s}, 3 \mathrm{H}), 2.24-2.22(\mathrm{~m}, 1 \mathrm{H})$, $1.48-1.44(\mathrm{~m}, 1 \mathrm{H}) ;{ }^{13} \mathrm{C} \mathrm{NMR}\left(100 \mathrm{MHz}, \mathrm{CDCl}_{3}\right): \delta 159.7,139.5,133.0,131.8,128.6,128.3,125.8,121.9,115.4,114.0$, 100.3, 93.6, 85.4, 67.7, 55.3, 25.8; IR (ATR) $\mathrm{cm}^{-1}:$ 2955, 2932, 2851, 2213, 1601, 1568, 1511, 1451, 1432, 1395, 1369, 1340, 1287, 1274, 1248, 1233, 1206, 1174, 1147, 1104, 1085, 1028, 1004; ESI-HRMS m/z: 317.1148 ([M+Na $\left.]^{+}\right)$; Calcd. for $\mathrm{C}_{19} \mathrm{H}_{18} \mathrm{O}_{3} \mathrm{Na}: 317.1148$.

2-(2-((4-Methoxyphenyl)ethynyl)phenyl)-5,5-dimethyl-1,3-dioxane (1r) 


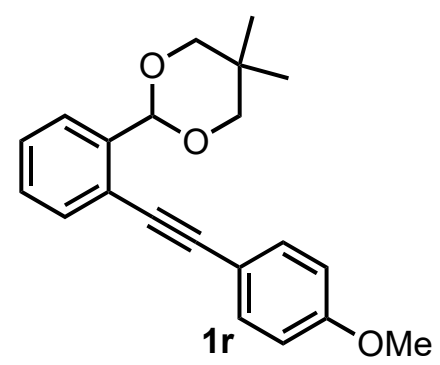

According to GP B, 2-((4-methoxyphenyl)ethynyl)-benzaldehyde (S1a, $354.4 \mathrm{mg}, 1.5 \mathrm{mmol})$, neopenthyl glycol (1.56 g, $15.0 \mathrm{mmol}), \mathrm{CH}(\mathrm{OMe})_{3}(262.0 \mu \mathrm{L}, 2.3 \mathrm{mmol})$, TBATB $(7.2 \mathrm{mg}, 15.0 \mu \mathrm{mol})$ and $\mathrm{THF}(5 \mathrm{~mL})$ were stirred for $24 \mathrm{~h}$ at room temperature. Purification by flash silica-gel column chromatography (hexane / ethyl acetate = 10 / 1) to give 2-(2-((4-methoxyphenyl)ethynyl)phenyl)-5,5-dimethyl-1,3-dioxane (1r, $145.1 \mathrm{mg}, 0.5 \mathrm{mmol})$ in $30 \%$ yield as a pale yellow oil.

${ }^{1} \mathrm{H}$ NMR (400 MHz, $\left.\mathrm{CDCl}_{3}\right): \delta 7.74(\mathrm{~d}, J=8.4,1 \mathrm{H}), 7.52-7.46(\mathrm{~m}, 3 \mathrm{H}), 7.39-7.29(\mathrm{~m}, 2 \mathrm{H}), 6.90(\mathrm{~d}, J=8.4 \mathrm{~Hz}, 2 \mathrm{H}), 5.87$ $(\mathrm{s}, 1 \mathrm{H}), 3.84(\mathrm{~s}, 3 \mathrm{H}), 3.82-3.67(\mathrm{~m}, 4 \mathrm{H}), 1.34(\mathrm{~s}, 3 \mathrm{H}), 0.82(\mathrm{~s}, 3 \mathrm{H}) ;{ }^{13} \mathrm{C} \mathrm{NMR}\left(100 \mathrm{MHz}, \mathrm{CDCl}_{3}\right): \delta 159.7,139.3,133.0$, $131.9,128.6,128.3,125.9,121.9,115.4,114.0,100.2,93.5,85.4,77.9,55.3,30.3,23.1,21.8 ;$ IR (ATR) $\mathrm{cm}^{-1}: 2954,2838$, $2215,1601,1570,1510,1465,1390,1286,1247,1214,1174,1107,1086,1029,1016,1086,1029,1016$; ESI-HRMS m/z: $345.1467\left([\mathrm{M}+\mathrm{Na}]^{+}\right)$; Calcd. for $\mathrm{C}_{21} \mathrm{H}_{22} \mathrm{O}_{3} \mathrm{Na}: 345.1461$.

\section{2-(2-Ethynylphenyl)-1,3-dioxolane (1s)}<smiles>C#Cc1ccccc1C1OCCO1</smiles>

$1 \mathrm{~s}$

According to GP B, 2-ethynylbenzaldehyde (S1j-2, $271.8 \mathrm{mg}, 1.6 \mathrm{mmol})$, ethylene glycol (892.0 $\mu \mathrm{L}, 16.0 \mathrm{mmol})$, $\mathrm{CH}(\mathrm{OMe})_{3}(263.0 \mu \mathrm{L}, 2.4 \mathrm{mmol})$, TBATB $(23.1 \mathrm{mg}, 18.0 \mu \mathrm{mol})$ and THF $(2 \mathrm{~mL})$ were stirred for $6 \mathrm{~h}$ at room temperature. Purification by flash silica-gel column chromatography (hexane / ethyl acetate = 20 / 1) to give 2-(2-ethynylphenyl)-1,3-dioxolane (1s, $167.2 \mathrm{mg}, 1.0 \mathrm{mmol})$ in $60 \%$ yield as a orangish oil.

${ }^{1} \mathrm{H}$ NMR (400 MHz, $\left.\mathrm{CDCl}_{3}\right): \delta 7.59(\mathrm{dd}, J=1.2,8.0 \mathrm{~Hz}, 1 \mathrm{H}), 7.53(\mathrm{dd}, J=1.2,7.6 \mathrm{~Hz}, 1 \mathrm{H}), 7.41-7.31(\mathrm{~m}, 2 \mathrm{H}), 6.22(\mathrm{~s}$, 1H), 4.21-4.13 (m, 2H), 4.12-4.04 (m, 2H), $3.33(\mathrm{~s}, 1 \mathrm{H})$. Spectroscopic data of ${ }^{1} \mathrm{H}$ NMR was identical to that of the reference 18 .

\section{((2-(1,3-Dioxolan-2-yl)phenyl)ethynyl)trimethylsilane (1t)}<smiles>CC(C)(C)C#Cc1ccccc1C1OCCO1</smiles>

$1 t$

According to GP B, 2-((trimethylsilyl)ethynyl)benzaldehyde (S1j-1, $323.7 \mathrm{mg}, 1.6 \mathrm{mmol})$ ethylene glycol (892.0 $\mu \mathrm{L}, 16.0$ $\mathrm{mmol}), \mathrm{CH}(\mathrm{OMe})_{3}(263.0 \mu \mathrm{L}, 2.4 \mathrm{mmol}), \mathrm{TBATB}(23.1 \mathrm{mg}, 18.0 \mu \mathrm{mol})$ and $\mathrm{THF}(2 \mathrm{~mL})$ were stirred for $6 \mathrm{~h}$ at room temperature. Purification by flash silica-gel column chromatography (hexane / ethyl acetate = 20 / 1) to give ((2-(1,3-dioxolan-2-yl)phenyl)ethynyl)trimethylsilane (1t, $264.1 \mathrm{mg}, 1.1 \mathrm{mmol})$ in 67\% yield as a pale yellow oil. 
${ }^{1} \mathrm{H}$ NMR (400 MHz, $\mathrm{CDCl}_{3}$ ): $\delta 7.55(\mathrm{~d}, J=7.6 \mathrm{~Hz}, 1 \mathrm{H}), 7.49$ (d, $\left.J=7.2 \mathrm{~Hz}, 1 \mathrm{H}\right), 7.37-7.28(\mathrm{~m}, 2 \mathrm{H}), 6.18(\mathrm{~s}, 1 \mathrm{H})$, 4.23-4.15 (m, 2H), 4.10-4.02 (m, 2H), $0.26(\mathrm{~s}, 9 \mathrm{H})$ Spectroscopic data of ${ }^{1} \mathrm{H}$ NMR was identical to that of the reference 18.

1-(Dimethoxymethyl)-2-((4-methoxyphenyl)ethynyl)benzene (4)

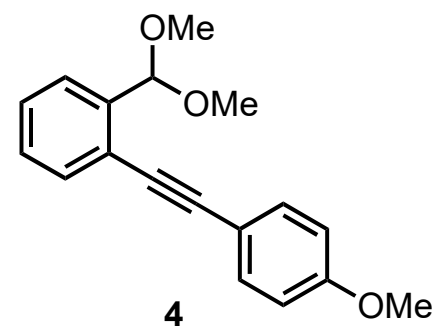

According to GP B, 2-((4-methoxyphenyl)ethynyl)-benzaldehyde (S1a, 378.0 mg, $1.6 \mathrm{mmol}), \mathrm{MeOH}(647.0 \mu \mathrm{L}, 16.0$ $\mathrm{mmol}), \mathrm{CH}(\mathrm{OMe})_{3}(263.0 \mu \mathrm{L}, 2.4 \mathrm{mmol})$, TBATB $(23.1 \mathrm{mg}, 18.0 \mu \mathrm{mol})$ and THF $(2 \mathrm{~mL})$ were stirred for $6 \mathrm{~h}$ at room temperature. Purification by flash silica-gel column chromatography (hexane / ethyl acetate $=20 / 1$ ) to give 1-(dimethoxymethyl)-2-((4-methoxyphenyl)ethynyl)benzene $(4,316.2 \mathrm{mg}, 1.1 \mathrm{mmol})$ in $70 \%$ yield as a pale yellow oil.

${ }^{1} \mathrm{H}$ NMR (400 MHz, $\mathrm{CDCl}_{3}$ ): $\delta 7.60$ (d, J = 7.2 Hz, 1H), 7.54-7.47 (m, 3H), 7.37-7.29 (m, 2H), 6.89 (d, J=8.8 Hz, 2H), $5.75(\mathrm{~s}, 1 \mathrm{H}), 3.84(\mathrm{~s}, 3 \mathrm{H}), 3.45(\mathrm{~s}, 6 \mathrm{H})$. Spectroscopic data of ${ }^{1} \mathrm{H}$ NMR was identical to that of the reference 19.

\section{1-(2-((4-Methoxyphenyl)ethynyl)phenyl)ethanone (S5)}

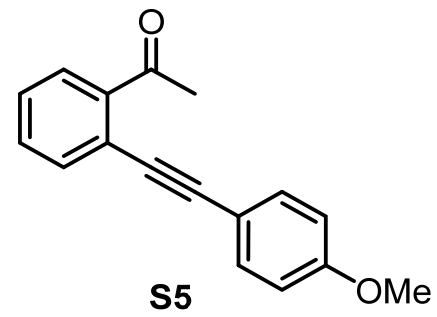

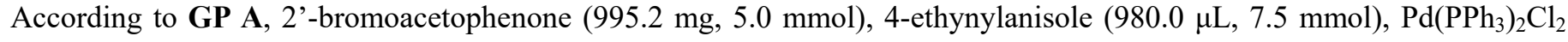
$(35.1 \mathrm{mg}, 50.0 \mu \mathrm{mol}), \mathrm{CuI}(19.0 \mathrm{mg}, 100.0 \mu \mathrm{mol}), \mathrm{Et}_{3} \mathrm{~N}(10 \mathrm{~mL})$ and THF $(5 \mathrm{~mL})$ were stirred for $24 \mathrm{~h}$ at $90{ }^{\circ} \mathrm{C}$ in oil bath. Purification by flash silica-gel column chromatography (hexane / ethyl acetate $=15 / 1$ ) to give 1-(2-((4-methoxyphenyl)ethynyl)phenyl)ethanone (S5, $1.0 \mathrm{~g}, 4.1 \mathrm{mmol})$ in $82 \%$ yield as a orangish oil.

${ }^{1} \mathrm{H}$ NMR $\left(400 \mathrm{MHz}, \mathrm{CDCl}_{3}\right): \delta 7.75(\mathrm{~d}, J=8.4 \mathrm{~Hz}, 1 \mathrm{H}), 7.61(\mathrm{~d}, J=7.6 \mathrm{~Hz}, 1 \mathrm{H}), 7.51-7.44(\mathrm{~m}, 3 \mathrm{H}), 7.37(\mathrm{t}, J=7.6 \mathrm{~Hz}$, $1 \mathrm{H}), 6.90(\mathrm{~d}, J=8.4 \mathrm{~Hz}, 2 \mathrm{H}), 3.84(\mathrm{~s}, 3 \mathrm{H}), 2.80(\mathrm{~s}, 3 \mathrm{H})$. Spectroscopic data of ${ }^{1} \mathrm{H}$ NMR was identical to that of the reference 20 .

2-(2-((4-methoxyphenyl)ethynyl)phenyl)-2-methyl-1,3-dioxolane (6)

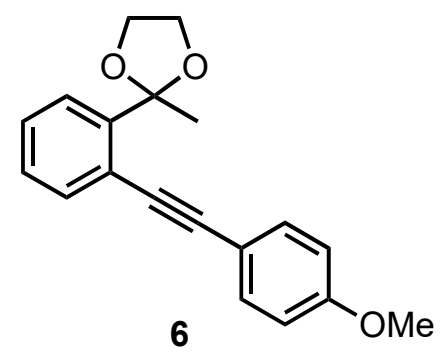

According to GP B, 1-(2-((4-methoxyphenyl)ethynyl)phenyl)ethanone (S5, $375.4 \mathrm{mg}, 1.5 \mathrm{mmol})$, ethylene glycol (4 $\mathrm{mL})$, $\mathrm{CH}(\mathrm{OMe})_{3}(328.0 \mu \mathrm{L}, 3.0 \mathrm{mmol})$, conc. $\mathrm{HCl}(1 \mathrm{drop})$ and THF $(4 \mathrm{~mL})$ were stirred for $24 \mathrm{~h}$ at room temperature. Purification by flash silica-gel column chromatography (hexane / ethyl acetate $=5 / 1$ ) to give 2-(2-((4-methoxyphenyl)ethynyl)phenyl)-2-methyl-1,3-dioxolane $(6,290.6 \mathrm{mg}, 1.0 \mathrm{mmol})$ in 66\% yield as a colorless solid. MP: $73-74{ }^{\circ} \mathrm{C} ;{ }^{1} \mathrm{H}$ NMR $\left(400 \mathrm{MHz}, \mathrm{CDCl}_{3}\right): \delta 7.63-7.60(\mathrm{~m}, 1 \mathrm{H}), 7.56-7.53(\mathrm{~m}, 1 \mathrm{H}), 7.51(\mathrm{~d}, J=8.8 \mathrm{~Hz}, 2 \mathrm{H}), 7.29-7.26$ 
$(\mathrm{m}, 2 \mathrm{H}), 6.89(\mathrm{~d}, J=8.8 \mathrm{~Hz}, 2 \mathrm{H}), 4.15-3.98(\mathrm{~m}, 2 \mathrm{H}), 3.87-3.77(\mathrm{~m}, 5 \mathrm{H}), 1.90(\mathrm{~s}, 3 \mathrm{H}) ;{ }^{13} \mathrm{C} \mathrm{NMR}(100 \mathrm{MHz}, \mathrm{CDCl}): \delta$ $159.5,144.0,133.9,132.9,127.7,127.5,125.8,121.1,115.9,113.9,108.9,93.8,87.7,64.4,55.3,25.6 ;$ IR $\left.^{(A T R}\right) \mathrm{cm}^{-1}$ : 2991, 2966, 2932, 2887, 2834, 2213, 1605, 1565, 1509, 1436, 1365, 1287, 1251, 1218, 1194, 1172, 1148, 1102, 1063, 1034, 1023; ESI-HRMS m/z: $317.1155\left([\mathrm{M}+\mathrm{Na}]^{+}\right)$; Calcd. for $\mathrm{C}_{19} \mathrm{H}_{18} \mathrm{O}_{3} \mathrm{Na}: 317.1148$.

\section{1-(1,1-Dimethoxyethyl)-2-((4-methoxyphenyl)ethynyl)benzene (7)}

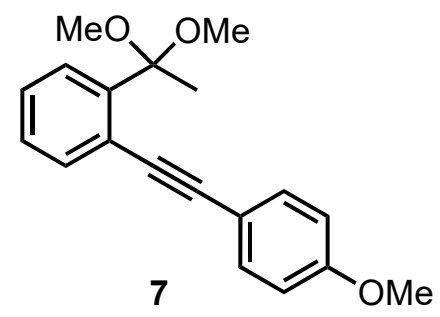

According to GP B, 1-(2-((4-methoxyphenyl)ethynyl)phenyl)ethanone (S5, $400.5 \mathrm{mg}, 1.6 \mathrm{mmol}), \mathrm{MeOH}(647.0 \mu \mathrm{L}, 16.0$ $\mathrm{mmol}), \mathrm{CH}(\mathrm{OMe})_{3}(263.0 \mu \mathrm{L}, 2.4 \mathrm{mmol})$, TBATB $(23.1 \mathrm{mg}, 18.0 \mu \mathrm{mol})$ and $\mathrm{THF}(2 \mathrm{~mL})$ were stirred for $6 \mathrm{~h}$ at room temperature. Purification by flash silica-gel column chromatography (hexane / ethyl acetate = 20/ 1) to give 1-(1,1-dimethoxyethyl)-2-((4-methoxyphenyl)ethynyl)benzene (7, $213.4 \mathrm{mg}, 720.0 \mu \mathrm{mol})$ in $45 \%$ yield as a pale yellow solid.

MP: $65-66{ }^{\circ} \mathrm{C} ;{ }^{1} \mathrm{H}$ NMR $\left(400 \mathrm{MHz}, \mathrm{CDCl}_{3}\right): \delta 7.71(\mathrm{dd}, J=1.9,7.3 \mathrm{~Hz}, 1 \mathrm{H}), 7.55(\mathrm{dd}, J=1.9,7.3 \mathrm{~Hz}, 1 \mathrm{H}), 7.48(\mathrm{~d}, J=8.6$ $\mathrm{Hz}, 2 \mathrm{H}), 7.32-7.24(\mathrm{~m}, 2 \mathrm{H}), 6.88(\mathrm{~d}, J=8.6 \mathrm{~Hz}, 2 \mathrm{H}), 3.83(\mathrm{~s}, 3 \mathrm{H}), 3.25(\mathrm{~s}, 6 \mathrm{H}), 1.74(\mathrm{~s}, 3 \mathrm{H}) ;{ }^{13} \mathrm{C} \mathrm{NMR}(100 \mathrm{MHz}, \mathrm{CDCl})$ : $\delta$ 159.4, 143.6, 133.8, 132.9, 132.8, 127.6, 127.4, 121.1, 116.0, 113.9, 101.5, 93.2, 87.7, 55.2, 48.8, 23.6; IR (ATR) $\mathrm{cm}^{-1}$ : 3065, 2995, 2958, 2936, 2827, 2214, 1191, 1685, 1605, 1564, 1507, 1441, 1371, 1287, 1245, 1184, 1175, 1142, 1107, 1074, 1048, 1025; ESI-HRMS m/z: $319.1310\left([\mathrm{M}+\mathrm{Na}]^{+}\right)$; Calcd. for $\mathrm{C}_{19} \mathrm{H}_{20} \mathrm{O}_{3} \mathrm{Na}: 319.1305$.

m-Methoxybenzylalcohol- $d_{2}\left(S 1 j-1-d_{2}\right)$<smiles></smiles>

To a solution of $m$-methoxybenzaldehyde $(1.1 \mathrm{~g}, 6.5 \mathrm{mmol})$ in 2-PrOH $(70 \mathrm{~mL})$ was added $\mathrm{NaBD}_{4}(408.1 \mathrm{mg}, 9.8 \mathrm{mmol})$ at room temperature under argon and stirred for $24 \mathrm{~h}$. The reaction mixture was quenched with $\mathrm{H}_{2} \mathrm{O}$ and extracted with ethyl acetate. The combined organic layers were dried over $\mathrm{Na}_{2} \mathrm{SO}_{4}$ and concentrated in vacuo. The residue was purified by silica-gel column chromatography (hexane / ethyl acetate $=7 / 1)$ to give $m$-methoxybenzylalcohol- $d_{2}\left(\mathbf{S 1} \mathbf{j}-\mathbf{1}-\boldsymbol{d}_{\mathbf{2}}, 1.0 \mathrm{~g}, 6.2\right.$ mmol) in $96 \%$ yield as a pale yellow oil.

${ }^{1} \mathrm{H}$ NMR (400 MHz, $\left.\mathrm{CDCl}_{3}\right): \delta 7.26(\mathrm{dd}, J=8.0,8.0 \mathrm{~Hz}, 1 \mathrm{H}), 6.92-6.90(\mathrm{~m}, 2 \mathrm{H}), 6.84-6.81(\mathrm{~m}, 1 \mathrm{H}), 4.63-4.61(\mathrm{~m}, 0.10 \mathrm{H})$, $3.79(\mathrm{~s}, 3 \mathrm{H}) ;{ }^{2} \mathrm{H} \mathrm{NMR}\left(77 \mathrm{MHz}, \mathrm{CHCl}_{3}\right): \delta 4.64$ (brs).

\section{m-Methoxybenzaldehyde- $d_{1}\left(\right.$ S1j-2- $\left.d_{1}\right)$}<smiles>COc1cccc(C(=O)O[Na])c1</smiles>

$$
\text { S1j-2-d }
$$

To a solution of $m$-methoxybenzylalcohol- $d_{2}\left(\mathbf{S} 1 \mathbf{j}-\mathbf{1}-\mathbf{d}_{\mathbf{2}}, 770.3 \mathrm{mg}, 5.5 \mathrm{mmol}\right)$ in $\mathrm{CH}_{2} \mathrm{Cl}_{2}(50 \mathrm{~mL})$ was added PDC (4.1 g, 
$11.0 \mathrm{mmol}$ ) at room temperature under argon and stirred for $24 \mathrm{~h}$. The reaction mixture was filtered through celite pad with $\mathrm{CH}_{2} \mathrm{Cl}_{2}$. The filtrate was concentrated in vacuo. The residue was purified by silica-gel column chromatography (hexane / ethyl acetate $=7 / 1)$ to give $m$-methoxybenzaldehyde- $d_{1}\left(\mathbf{S 1} \mathbf{j}-\mathbf{2}-\mathbf{d}_{\mathbf{1}}, 614.7 \mathrm{mg}, 4.7 \mathrm{mmol}\right)$ in $85 \%$ yield as a pale yellow oil. ${ }^{1} \mathrm{H}$ NMR (400 MHz, $\left.\mathrm{CDCl}_{3}\right): \delta 9.99$ (s, 0.01H), 7.49-7.40 (m, 3H), 7.22-7.17 (m, 1H), 3.88 (s, 3H); ${ }^{2} \mathrm{H} \mathrm{NMR}(61 \mathrm{MHz}$, $\left.\mathrm{CHCl}_{3}\right): \delta 10.01$ (brs).

\section{2-Bromo-5-methoxybenzaldehyde- $d_{1}\left(S 1 j-3-d_{1}\right)$}<smiles>COc1ccc(Br)c(C(=O)O[Na])c1</smiles>

S1j-3-d

To a solution of $m$-methoxybenzaldehyde- $d_{1}\left(\mathbf{S} 1 \mathbf{j}-\mathbf{2}-\boldsymbol{d}_{\mathbf{1}}, 548.6 \mathrm{mg}, 4.0 \mathrm{mmol}\right)$ in DMF $(5 \mathrm{~mL})$ was added NBS $(854.3 \mathrm{mg}$, $4.8 \mathrm{mmol}$ ) at room temperature under argon and stirred for $12 \mathrm{~h}$. To the reaction mixture was added $\mathrm{H}_{2} \mathrm{O}$ at $0{ }^{\circ} \mathrm{C}$. The precipitate was collected by filtration, washed with $\mathrm{H}_{2} \mathrm{O}(100 \mathrm{~mL})$, and dried under vacuum to give 2-bromo-5-methoxybenzaldehyde- $d_{1}\left(\mathbf{S} 1 \mathbf{j}-\mathbf{3}-\mathbf{d}_{\mathbf{1}}, 655.3 \mathrm{mg}, 3.0 \mathrm{mmol}\right)$ in $76 \%$ yield as a pale yellow solid.

${ }^{1} \mathrm{H}$ NMR (400 MHz, $\left.\mathrm{CDCl}_{3}\right): \delta 10.32(\mathrm{~s}, 0.01 \mathrm{H}), 7.53(\mathrm{~d}, J=8.8 \mathrm{~Hz}, 1 \mathrm{H}), 7.43(\mathrm{~d}, J=3.2 \mathrm{~Hz}, 1 \mathrm{H}), 7.04(\mathrm{dd}, J=3.2,8.8 \mathrm{~Hz}$, 1H), 3.85 (s, 3H); ${ }^{2} \mathrm{H}$ NMR (61 MHz, $\mathrm{CHCl}_{3}$ ): $\delta 10.34$ (brs).

\section{5-Methoxy-2-((4-methoxyphenyl)ethynyl)benzaldehyde- $d_{1}\left(S 1 j-d_{1}\right)$}

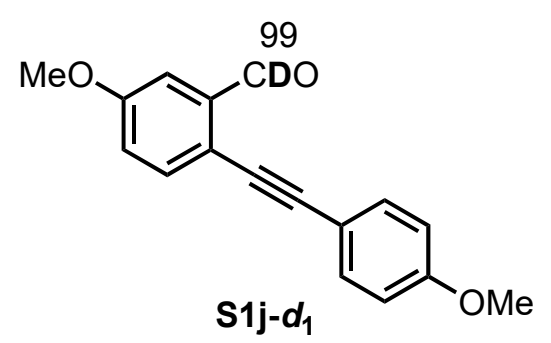

According to GP A, 2-bromo-5-methoxybenzaldehyde- $\boldsymbol{d}_{1}\left(\mathbf{S 1 j} \mathbf{j}-\mathbf{3 - d _ { \mathbf { 1 } }}, 602.1 \mathrm{mg}, 2.8 \mathrm{mmol}\right)$, 4-ethynylanisole (436.0 $\mu \mathrm{L}, 3.4$ $\mathrm{mmol}), \mathrm{Pd}\left(\mathrm{PPh}_{3}\right)_{2} \mathrm{Cl}_{2}(98.3 \mathrm{mg}, 140.0 \mu \mathrm{mol}), \mathrm{CuI}(26.7 \mathrm{mg}, 140.0 \mu \mathrm{mol}), \mathrm{Et}_{3} \mathrm{~N}(4 \mathrm{~mL})$ and THF $(6 \mathrm{~mL})$ were stirred for $2 \mathrm{~h}$ at $80{ }^{\circ} \mathrm{C}$ in oil bath. Purification by flash silica-gel column chromatography (hexane / ethyl acetate $=15 / 1$ ) to give 5-methoxy-2-((4-methoxyphenyl)ethynyl)benzaldehyde- $\boldsymbol{d}_{1}\left(\mathbf{S} 1 \mathbf{j}-\boldsymbol{d}_{\mathbf{1}}, 662.8 \mathrm{mg}, 2.5 \mathrm{mmol}\right)$ in $89 \%$ yield as a pale yellow solid.

${ }^{1} \mathrm{H}$ NMR $\left(400 \mathrm{MHz}, \mathrm{CDCl}_{3}\right): \delta 10.61(\mathrm{~s}, 0.01 \mathrm{H}), 7.54(\mathrm{~d}, J=8.2 \mathrm{~Hz}, 1 \mathrm{H}), 7.48(\mathrm{~d}, J=8.8 \mathrm{~Hz}, 2 \mathrm{H}), 7.43(\mathrm{~d}, J=2.8 \mathrm{~Hz}, 1 \mathrm{H})$, $7.14(\mathrm{dd}, J=2.8,8.2 \mathrm{~Hz}, 1 \mathrm{H}), 6.90(\mathrm{~d}, J=8.8 \mathrm{~Hz}, 2 \mathrm{H}), 3,88(\mathrm{~s}, 3 \mathrm{H}), 3.85(\mathrm{~s}, 3 \mathrm{H}) ;{ }^{2} \mathrm{H}$ NMR $\left(77 \mathrm{MHz}, \mathrm{CDCl}_{3}\right): \delta 10.63$ (brs).

\section{2-(5-Methoxy-2-((4-methoxyphenyl)ethynyl)phenyl)-1,3-dioxolane- $d_{1}\left(1 \mathrm{j}-d_{1}\right)$}

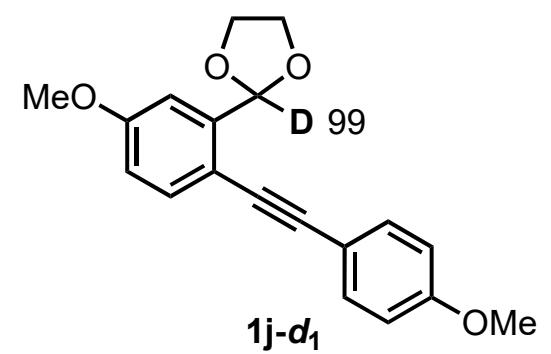

According to GP B, 5-methoxy-2-((4-methoxyphenyl)ethynyl)benzaldehyde- $\boldsymbol{d}_{1}$ (S1j- $\left.\boldsymbol{d}_{\mathbf{1}}, 532.6 \mathrm{mg}, 2.0 \mathrm{mmol}\right)$, ethylene glycol $(1.1 \mathrm{~mL}, 20.0 \mathrm{mmol}), \mathrm{CH}(\mathrm{OMe})_{3}(412.0 \mu \mathrm{L}, 4.0 \mathrm{mmol})$, TBATB $(28.9 \mathrm{mg}, 60.0 \mu \mathrm{mol})$ and THF $(2 \mathrm{~mL})$ were stirred 
for $12 \mathrm{~h}$ at room temperature. Purification by flash silica-gel column chromatography (hexane $/$ ethyl acetate $=15 / 1$ ) to give 2-(5-methoxy-2-((4-methoxyphenyl)ethynyl)phenyl)-1,3-dioxolane- $d_{1}\left(\mathbf{1} \mathbf{j}-\mathbf{d}_{\mathbf{1}}, 335.6 \mathrm{mg}, 1.1 \mathrm{mmol}\right)$ in $54 \%$ yield as a pale yellow solid.

${ }^{1} \mathrm{H}$ NMR (400 MHz, CDCl $): \delta 7.47-7.44(\mathrm{~m}, 3 \mathrm{H}), 7.13(\mathrm{~d}, J=2.4 \mathrm{~Hz}, 1 \mathrm{H}), 6.89-6.86(\mathrm{~m}, 3 \mathrm{H}), 6.24(\mathrm{~s}, 0.01 \mathrm{H}), 4.23-4.16$ (m, 2H), 4.11-4.05 (m, 2H), 3.84 (s, 3H), 3.83 (s, 3H); ${ }^{2} \mathrm{H}$ NMR (61 MHz, $\left.\mathrm{CHCl}_{3}\right): \delta 6.23$ (brs).

\section{1-(2-(4-Methoxyphenyl)ethynyl)-2-methylbenzene (8)}

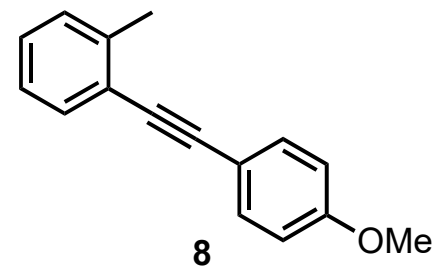

According to GP A, 2-iodoanisole (468.1 mg, $2.0 \mathrm{mmol}$ ), 2-ethynyltoluene (255.6 mg, $2.2 \mathrm{mmol}), \mathrm{Pd}\left(\mathrm{PPh}_{3}\right)_{2} \mathrm{Cl}_{2}(42.1 \mathrm{mg}$, $60.0 \mu \mathrm{mol}), \mathrm{CuI}(11.4 \mathrm{mg}, 60.0 \mu \mathrm{mol}), \mathrm{Et}_{3} \mathrm{~N}(3 \mathrm{~mL})$ and THF $(6 \mathrm{~mL})$ were stirred for $6 \mathrm{~h}$ at $80{ }^{\circ} \mathrm{C}$ in oil bath. Purification by flash silica-gel column chromatography (hexane / ethyl acetate $=40 / / 1$ ) to give 1-(2-(4-methoxyphenyl)ethynyl)-2-methylbenzene $(\mathbf{8}, 77.3 \mathrm{mg}, 0.4 \mathrm{mmol}) \mathrm{in} 62 \%$ yield as a brown solid.

${ }^{1} \mathrm{H}$ NMR (400 MHz, $\mathrm{CDCl}_{3}$ ): $\delta 7.52-7.46$ (m, 3H), 7.23-7.12 (m, 3H), 6.88 (d, J=8.4 Hz, 2H), 3.83 (s, 3H), 2.51 (s, 3H). Spectroscopic data of ${ }^{1} \mathrm{H}$ NMR was identical to that of the reference 21.

\section{2,5-Dibromo-1,4-benzendicarboxaldehyde (S10-1)}<smiles>O=Cc1cc(Br)c(C=O)cc1Br</smiles>

S10-1

To a solution of terepthalaldehyde $(4.0 \mathrm{~g}, 30.0 \mathrm{mmol})$ in conc. $\mathrm{H}_{2} \mathrm{SO}_{4}(40 \mathrm{~mL})$ was added NBS $(11.5 \mathrm{~g}, 65.0 \mathrm{mmol})$ at $60{ }^{\circ} \mathrm{C}$ under argon and stirred for $3 \mathrm{~h}$. To the reaction mixture was added $\mathrm{H}_{2} \mathrm{O}$ at $0{ }^{\circ} \mathrm{C}$. The precipitate was collected by filtration and dissolved in $\mathrm{CH}_{2} \mathrm{Cl}_{2}$. The organic layers were washed with saturated $\mathrm{NaHCO}_{3}$ aqueous solution, dried over $\mathrm{Na}_{2} \mathrm{SO}_{4}$ and concentrated in vacuo. The resudiue was purified crystallization with $\mathrm{CH}_{2} \mathrm{Cl}_{2}$ and hexane to give 2,5-dibromo-1,4-benzendicarboxaldehyde (S10-1, $4.4 \mathrm{~g}, 15.0 \mathrm{mmol})$ in 50\% yield as a pale yellow solid.

${ }^{1} \mathrm{H}$ NMR (400 MHz, $\left.\mathrm{CDCl}_{3}\right): \delta 10.35(\mathrm{~s}, 2 \mathrm{H}), 8.16(\mathrm{~s}, 2 \mathrm{H})$. Spectroscopic data of ${ }^{1} \mathrm{H}$ NMR was identical to that of the reference 22 .

\section{2,5-Bis((4-methoxyphenyl)ethynyl)terephtalaldehyde (S10-2)}

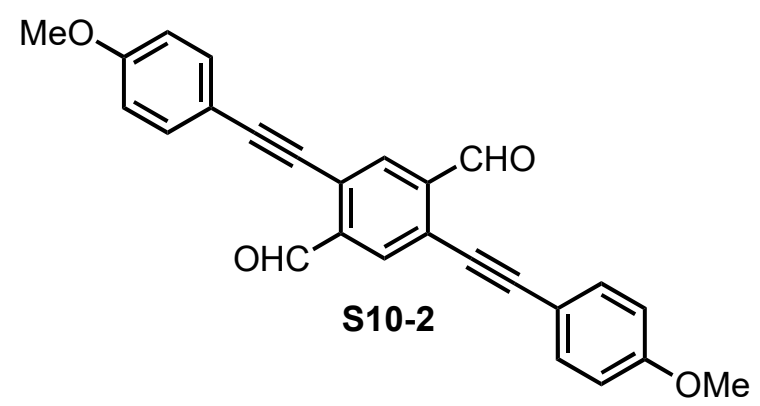

According to GP A, 2,5-dibromo-1,4-benzendicarboxaldehyde (S10-1, 583.9 mg, $2.0 \mathrm{mmol})$, 4-ethynylanisole (629.0 $\mu \mathrm{L}$, $4.8 \mathrm{mmol}), \mathrm{Pd}\left(\mathrm{PPh}_{3}\right)_{2} \mathrm{Cl}_{2}(42.1 \mathrm{mg}, 60.0 \mu \mathrm{mol}), \mathrm{CuI}(11.4 \mathrm{mg}, 60.0 \mu \mathrm{mol}), \mathrm{Et}_{3} \mathrm{~N}(4 \mathrm{~mL})$ and THF $(12 \mathrm{~mL})$ were stirred for 6 $\mathrm{h}$ at $80{ }^{\circ} \mathrm{C}$ in oil bath. Purification by crystallization with $\mathrm{CH}_{2} \mathrm{Cl}_{2}$ and hexane to give 2,5-bis((4-methoxyphenyl)ethynyl)terephtalaldehyde (S10-2, $394.4 \mathrm{mg}, 1.0 \mathrm{mmol})$ in 50\% yield as a yellow solid. 
MP: $222-223{ }^{\circ} \mathrm{C} ;{ }^{1} \mathrm{H}$ NMR $\left(400 \mathrm{MHz}, \mathrm{CDCl}_{3}\right): \delta 10.66(\mathrm{~s}, 2 \mathrm{H}), 8.15(\mathrm{~s}, 2 \mathrm{H}), 7.53(\mathrm{~d}, J=8.8 \mathrm{~Hz}, 4 \mathrm{H}), 6.93(\mathrm{~d}, J=8.8 \mathrm{~Hz}$, $4 \mathrm{H}), 3.86(\mathrm{~s}, 6 \mathrm{H}) ;{ }^{13} \mathrm{C} \mathrm{NMR}\left(100 \mathrm{MHz}, \mathrm{CDCl}_{3}\right): \delta 190.7,138.0,133.4,132.1,130.5,127.9,126.0,120.0,119.9,114.3$, 53.4; IR (ATR) $\mathrm{cm}^{-1}: 3366,2943,2844,2196,1686,1601,1566,1512,1467,1437,1410,1298,1276,1250,1192,1174$, 1145, 1129, 1110, 1026; ESI-HRMS m/z: $395.1206\left([\mathrm{M}+\mathrm{H}]^{+}\right)$; Calcd. for $\mathrm{C}_{26} \mathrm{H}_{19} \mathrm{O}_{4}: 395.1278$.

\section{2,2'-(2,5-Bis((4-methoxyphenyl)ethynyl)-1,4-phenylene)bis(1,3-dioxolane) (10)}

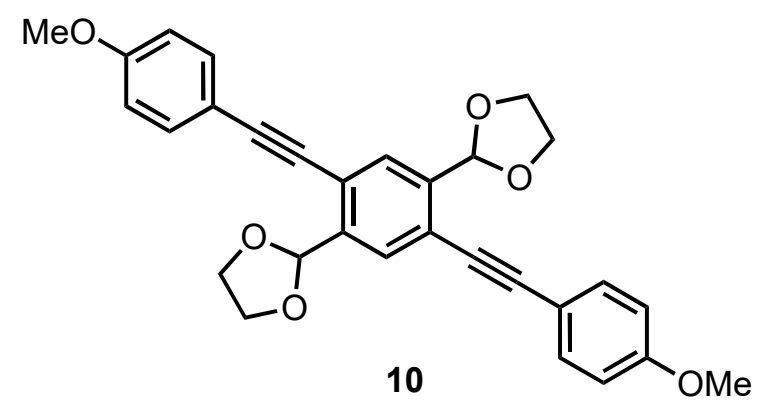

According to GP B, 5-methoxy-2-((4-methoxyphenyl)ethynyl)benzaldehyde- $d_{1}$ (S10-2, $\left.394.4 \mathrm{mg}, 1.0 \mathrm{mmol}\right)$, ethylene glycol $(1.7 \mathrm{~mL}, 30.0 \mathrm{mmol}), \mathrm{CH}(\mathrm{OMe})_{3}(328.0 \mu \mathrm{L}, 3.0 \mathrm{mmol})$, TBATB $(48.2 \mathrm{mg}, 100.0 \mu \mathrm{mol})$ and $\mathrm{CH}_{2} \mathrm{Cl}_{2}(10 \mathrm{~mL})$ were stirred for $12 \mathrm{~h}$ at room temperature. Purification by crystallization with $\mathrm{CH}_{2} \mathrm{Cl}_{2}$ and hexane to give 2,2'-(2,5-bis((4-methoxyphenyl)ethynyl)-1,4-phenylene)bis(1,3-dioxolane) $(\mathbf{1 0}, 106.3 \mathrm{mg}, 200.0 \mu \mathrm{mol})$ in $22 \%$ yield as a yellow solid.

MP: $223-224{ }^{\circ} \mathrm{C} ;{ }^{1} \mathrm{H}$ NMR $\left(400 \mathrm{MHz}, \mathrm{CDCl}_{3}\right): \delta 7.74$ (s, 2H), 7.48 (d, $\left.J=8.8 \mathrm{~Hz}, 4 \mathrm{H}\right), 6.89$ (d, $\left.J=8.8 \mathrm{~Hz}, 4 \mathrm{H}\right), 6.27$ (s, 2H), 4.24-4.16 (m, 4H), 4.13-4.05 (m, 4H), $\left.3.84(\mathrm{~s}, 6 \mathrm{H}) ;{ }^{13} \mathrm{C} \mathrm{NMR} \mathrm{(100} \mathrm{MHz,} \mathrm{CDCl}_{3}\right): \delta 159.8,139.5,133.1,130.2,122.4$, 115.1, 114.9, 114.0, 101.6, 95.6, 65.5, 55.3; IR (ATR) $\mathrm{cm}^{-1}: 2964,2890,2838,2212,1600,1509,1464,1437,1393,1286$, 1241, 1176, 1138, 1106, 1075, 1022; ESI-HRMS m/z: 505.1628 ([M+Na] $]^{+}$; Calcd. for $\mathrm{C}_{30} \mathrm{H}_{26} \mathrm{O}_{6} \mathrm{Na}$ : 505.1622 .

\section{Procedure for the synthesis of products}

\section{2'-(4-Methoxyphenyl)spiro[[1,3]dioxolane-2,1'-indene] (2a)}

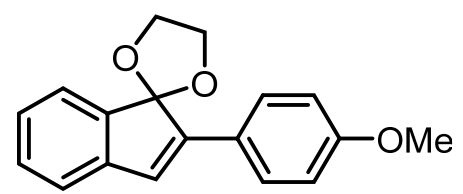

$2 \mathbf{a}$

According to GP C, 2-(2-((4-methoxyphenyl)ethynyl)phenyl)-1,3-dioxolane (1a, $28.0 \mathrm{mg}, 0.1 \mathrm{mmol}), \mathrm{IPrAuCl}$ (3.1 mg, 5.0 $\mu \mathrm{mol})$ and $\mathrm{AgSbF}_{6}(1.7 \mathrm{mg}, 5.0 \mu \mathrm{mol})$ were dissolved in dichloroethane $(1 \mathrm{~mL})$ and stirred for $6 \mathrm{~h}$ at $80{ }^{\circ} \mathrm{C}$ in oil bath. Purification by flash silica-gel column chromatography (hexane / ethyl acetate $=10 / 1$ ) to give 2'-(4-methoxyphenyl)spiro[[1,3]dioxolane-2,1'-indene] (2a, $28.0 \mathrm{mg}, 0.1 \mathrm{mmol})$ in quantitative yield as a pale yellow solid. MP: $148-149{ }^{\circ} \mathrm{C} ;{ }^{1} \mathrm{H}$ NMR $\left(400 \mathrm{MHz}, \mathrm{CDCl}_{3}\right): \delta 7.60(\mathrm{~d}, J=9.0 \mathrm{~Hz}, 2 \mathrm{H}), 7.28(\mathrm{~d}, J=7.4 \mathrm{~Hz}, 1 \mathrm{H}), 7.23(\mathrm{dd}, J=7.4,7.4$ $\mathrm{Hz}, 1 \mathrm{H}), 7.12(\mathrm{~d}, J=7.4 \mathrm{~Hz}, 2 \mathrm{H}), 6.90(\mathrm{~d}, J=9.0 \mathrm{~Hz}, 2 \mathrm{H}), 6.78(\mathrm{~s}, 1 \mathrm{H}), 4.47-4.39(\mathrm{~m}, 2 \mathrm{H}), 4.38-4.30(\mathrm{~m}, 2 \mathrm{H}), 3.83(\mathrm{~s}$, $3 \mathrm{H}) ;{ }^{13} \mathrm{C}$ NMR $\left(100 \mathrm{MHz}, \mathrm{CDCl}_{3}\right): \delta 159.5,145.2,144.8,140.6,129.6,128.8,128.1,126.6,126.4,121.6,121.4,115.3$, 113.8, 65.3, 55.2; IR (ATR) $\mathrm{cm}^{-1}:$ 3059, 2962, 2896, 2838, 1599, 1506, 1487, 1456, 1439, 1329, 1300, 1272, 1250, 1218 , 1182, 1140, 1111, 1084, 1022; ESI-HRMS m/z: $281.1163\left([\mathrm{M}+\mathrm{H}]^{+}\right)$; Calcd. for $\mathrm{C}_{18} \mathrm{H}_{17} \mathrm{O}_{3}: 281.1172$. 


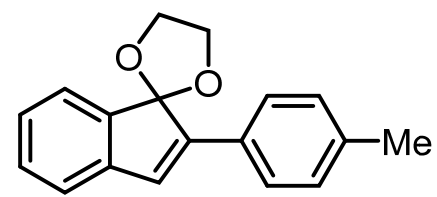

$\mathbf{2 b}$

According to GP C, 2-(2-(p-tolylethynyl)phenyl)-1,3-dioxolane (1b, $26.4 \mathrm{mg}, 0.1 \mathrm{mmol}), \operatorname{IPrAuCl}(3.1 \mathrm{mg}, 5.0 \mu \mathrm{mol})$ and $\mathrm{AgSbF}_{6}(1.7 \mathrm{mg}, 5.0 \mu \mathrm{mol})$ were dissolved in dichloroethane $(1 \mathrm{~mL})$ and stirred for $6 \mathrm{~h}$ at $80{ }^{\circ} \mathrm{C}$ in oil bath. Purification by flash silica-gel column chromatography (hexane $/$ ethyl acetate $=20 /$ ) to give 2'-(p-tolyl)spiro[[1,3]dioxolane-2,1'-indene] (2b, $24.6 \mathrm{mg}, 930.0 \mu \mathrm{mol})$ in $93 \%$ yield as a pale yellow solid.

MP: $76-77^{\circ} \mathrm{C} ;{ }^{1} \mathrm{H}$ NMR $\left(400 \mathrm{MHz}, \mathrm{CDCl}_{3}\right): \delta 7.55(\mathrm{~d}, J=8.4 \mathrm{~Hz}, 2 \mathrm{H}), 7.29(\mathrm{~d}, J=7.6 \mathrm{~Hz}, 1 \mathrm{H}), 7.22(\mathrm{~d}, J=6.8 \mathrm{~Hz}, 1 \mathrm{H})$, 7.19-7.11 (m, 4H), $6.82(\mathrm{~s}, 1 \mathrm{H}), 4.46-4.39(\mathrm{~m}, 2 \mathrm{H}), 4.37-4.31(\mathrm{~m}, 2 \mathrm{H}), 2.37(\mathrm{~s}, 3 \mathrm{H}) ;{ }^{13} \mathrm{C} \mathrm{NMR}\left(100 \mathrm{MHz}, \mathrm{CDCl}_{3}\right): \delta 145.4$, 140.5, 137.9, 131.2, 129.7, 129.6, 129.1, 128.7, 126.8, 126.6, 121.6, 121.6, 115.3, 65.4, 21.3; IR (ATR) $\mathrm{cm}^{-1}: 2959,2892$, 1734, 1601, 1508, 1454, 1352, 1327, 1292, 1224, 1206, 1188, 1177, 1140, 1082, 1031; ESI-HRMS m/z: 287.1017 $\left([\mathrm{M}+\mathrm{Na}]^{+}\right)$; Calcd. for $\mathrm{C}_{18} \mathrm{H}_{16} \mathrm{O}_{2} \mathrm{Na}: 287.1043$.

\section{2'-Phenylspiro[[1,3]dioxolane-2,1'-indene] (2c)}

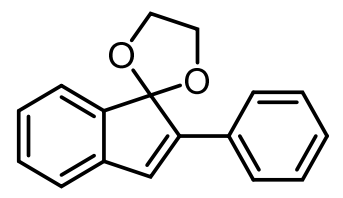

2c

According to GP C, 2-(2-(phenylethynyl)phenyl)-1,3-dioxolane (1c, $25.0 \mathrm{mg}, 0.1 \mathrm{mmol}), \mathrm{IPrAuCl}(3.1 \mathrm{mg}, 5.0 \mu \mathrm{mol})$ and $\mathrm{AgSbF}_{6}(1.7 \mathrm{mg}, 5.0 \mu \mathrm{mol})$ were dissolved in dichloroethane $(1 \mathrm{~mL})$ and stirred for $6 \mathrm{~h}$ at $80{ }^{\circ} \mathrm{C}$ in oil bath. Purification by flash silica-gel column chromatography (hexane / ethyl acetate $=20$ / 1) to give 2'-phenylspiro[[1,3]dioxolane-2,1'-indene] (2c, $18.5 \mathrm{mg}, 740.0 \mu \mathrm{mol})$ in $74 \%$ yield as a colorless solid.

MP: $105-106{ }^{\circ} \mathrm{C} ;{ }^{1} \mathrm{H}$ NMR $\left(400 \mathrm{MHz}, \mathrm{CDCl}_{3}\right): \delta 7.65(\mathrm{~d}, J=7.6 \mathrm{~Hz}, 2 \mathrm{H}), 7.38-7.35(\mathrm{~m}, 2 \mathrm{H}), 7.33-7.30(\mathrm{~m}, 2 \mathrm{H})$, $7.27-7.23(\mathrm{~m}, 2 \mathrm{H}), 7.16(\mathrm{~d}, J=7.6 \mathrm{~Hz}, 2 \mathrm{H}), 6.87(\mathrm{~s}, 1 \mathrm{H}), 4.47-4.39(\mathrm{~m}, 2 \mathrm{H}), 4.37-4.29(\mathrm{~m}, 2 \mathrm{H}) ;{ }^{13} \mathrm{C} \mathrm{NMR}(100 \mathrm{MHz}$, $\mathrm{CDCl}_{3}$ ): $\delta 145.4,145.4,140.4,134.1,130.5,129.6,128.3,128.0,126.9,126.8,121.7,121.7,115.4,65.4$; IR (ATR) $\mathrm{cm}^{-1}$ : 3044, 2975, 2903, 1601, 1492, 1455, 1341, 1299, 1220, 1204, 1182, 1141, 1083, 1036, 1026; ESI-HRMS m/z: 251.1061 $\left([\mathrm{M}+\mathrm{H}]^{+}\right)$; Calcd. for $\mathrm{C}_{17} \mathrm{H}_{15} \mathrm{O}: 251.1067$.

\section{2'-(4-Fluorophenyl)spiro[[1,3]dioxolane-2,1'-indene] (2d)}

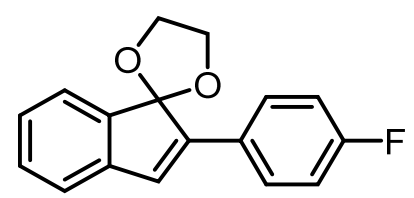

2d

According to GP C, 2-(2-((4-fluorophenyl)ethynyl)phenyl)-1,3-dioxolane (1d, $26.8 \mathrm{mg}, 0.1 \mathrm{mmol}), \operatorname{IPrAuCl}(3.1 \mathrm{mg}, 5.0$ $\mu \mathrm{mol})$ and $\mathrm{AgSbF}_{6}(1.7 \mathrm{mg}, 5.0 \mu \mathrm{mol})$ were dissolved in dichloroethane $(1 \mathrm{~mL})$ and stirred for $6 \mathrm{~h}$ at $80{ }^{\circ} \mathrm{C}$ in oil bath. Purification by flash silica-gel column chromatography (hexane / ethyl acetate $=20 / 1$ ) to give 2'-(4-fluorophenyl)spiro[[1,3]dioxolane-2,1'-indene] (2d, $17.4 \mathrm{mg}, 650.0 \mu \mathrm{mol})$ in 65\% yield as a yellow solid.

MP: $58-59^{\circ} \mathrm{C} ;{ }^{1} \mathrm{H}$ NMR $\left(500 \mathrm{MHz}, \mathrm{CDCl}_{3}\right): \delta 7.65-7.60(\mathrm{~m}, 2 \mathrm{H}), 7.30(\mathrm{~d}, J=8.0 \mathrm{~Hz}, 1 \mathrm{H}), 7.26-7.23(\mathrm{~m}, 1 \mathrm{H}), 7.16-7.13$ $(\mathrm{m}, 2 \mathrm{H}), 7.08-7.03(\mathrm{~m}, 2 \mathrm{H}), 6.82(\mathrm{~s}, 1 \mathrm{H}), 4.47-4.40(\mathrm{~m}, 2 \mathrm{H}), 4.35-4.28(\mathrm{~m}, 2 \mathrm{H}) ;{ }^{13} \mathrm{C} \mathrm{NMR}\left(100 \mathrm{MHz}, \mathrm{CDCl}_{3}\right): \delta 162.6(\mathrm{~d}$, 
$J=247.2 \mathrm{~Hz}), 145.2,144.1,140.2,130.3,130.1,130.1,129.7,128.6(\mathrm{~d}, J=7.7 \mathrm{~Hz}), 126.9,126.8,121.7(\mathrm{~d}, J=5.8 \mathrm{~Hz})$, $115.3(\mathrm{~d}, J=21.1 \mathrm{~Hz}), 65.4$; IR (ATR) $\mathrm{cm}^{-1}: 2925,1713,1600,1508,1461,1260,1215,1158,1093,1015$; ESI-HRMS m/z: $269.0971\left([\mathrm{M}+\mathrm{H}]^{+}\right)$; Calcd. for $\mathrm{C}_{17} \mathrm{H}_{14} \mathrm{O}_{2} \mathrm{~F}: 269.0972$.

Methyl 4-(spiro[[1,3]dioxolane-2,1'-inden]-2'-yl)benzoate (2e)

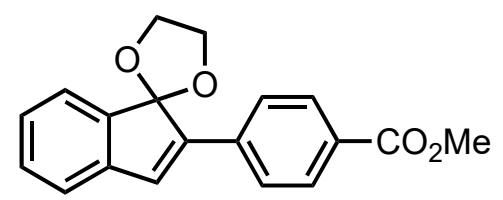

$2 e$

According to GP C, methyl 4-((2-(1,3-dioxolan-2-yl)phenyl)ethynyl)benzoate (1e, $30.8 \mathrm{mg}, 0.1 \mathrm{mmol})$, and $\operatorname{IPrAuSbF}_{6}(4.1$ $\mathrm{mg}, 5.0 \mu \mathrm{mol})$ were dissolved in dichloroethane $(1 \mathrm{~mL})$ and stirred for $3 \mathrm{~h}$ at $80{ }^{\circ} \mathrm{C}$ in oil bath. Purification by flash silica-gel column chromatography (hexane / ethyl acetate $=10 /$ 1) to give methyl 4-(spiro[[1,3]dioxolane-2,1'-inden]-2'-yl)benzoate (2e, $22.8 \mathrm{mg}, 740.0 \mu \mathrm{mol})$ in $74 \%$ yield as a coloeless solid.

MP: $137-138^{\circ} \mathrm{C} ;{ }^{1} \mathrm{H}$ NMR (400 MHz, $\left.\mathrm{CDCl}_{3}\right): \delta 8.03(\mathrm{~d}, J=8.4 \mathrm{~Hz}, 2 \mathrm{H}), 7.72(\mathrm{~d}, J=8.4 \mathrm{~Hz}, 2 \mathrm{H}), 7.32(\mathrm{~d}, J=7.2 \mathrm{~Hz}, 1 \mathrm{H})$, 7.27-7.25 (m, 1H), 7.20-7.18 (m, 2H), 4.49-4.41 (m, 2H), 4.39-4.31 (m, 2H), $3.92(\mathrm{~s}, 3 \mathrm{H}) ;{ }^{13} \mathrm{C} \mathrm{NMR}\left(100 \mathrm{MHz}, \mathrm{CDCl}_{3}\right): \delta$ 167.0, 145.6, 144.2, 139.9, 138.6, 132.5, 129.8, 129.7, 129.3, 127.4, 126.8, 122.3, 121.9, 115.4, 65.5, 52.2; IR (ATR) $\mathrm{cm}^{-1}$ : $3065,2950,2919,2896,2848,1714,1605,1556,1455,1434,1411,1311,1280,1237,1221,1191,1143,1108,1083,1032$, 1016; ESI-HRMS m/z: $309.1133\left([\mathrm{M}+\mathrm{H}]^{+}\right)$; Calcd. for $\mathrm{C}_{19} \mathrm{H}_{17} \mathrm{O}_{4}: 309.1121$.

2'-(o-Tolyl)spiro[[1,3]dioxolane-2,1'-indene] (2f)

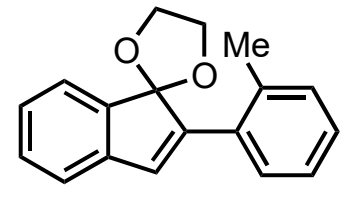

$2 f$

According to GP C, 2-(2-(o-tolylethynyl)phenyl)-1,3-dioxolane (1f, $26.4 \mathrm{mg}, 0.1 \mathrm{mmol})$ and $\operatorname{IPrAuSbF}_{6}(4.1 \mathrm{mg}, 5.0 \mu \mathrm{mol})$ were dissolved in dichloroethane $(1 \mathrm{~mL})$ and stirred for $6 \mathrm{~h}$ at $80^{\circ} \mathrm{C}$ in oil bath. Purification by flash silica-gel column chromatography (hexane / ethyl acetate $=20 / 1)$ to give 2'-(o-tolyl)spiro[[1,3]dioxolane-2,1'-indene] $(\mathbf{2} \mathbf{f}, 26.4 \mathrm{mg}, 0.1$ mmol) in quantitative yield as a yellow oil.

${ }^{1} \mathrm{H}$ NMR $\left(400 \mathrm{MHz}, \mathrm{CDCl}_{3}\right): \delta 7.46(\mathrm{~d}, J=7.6 \mathrm{~Hz}, 1 \mathrm{H}), 7.32-7.12(\mathrm{~m}, 8 \mathrm{H}), 6.45(\mathrm{~s}, 1 \mathrm{H}), 4.20-4.12(\mathrm{~m}, 2 \mathrm{H}), 3.83-3.74(\mathrm{~m}$, 2H), 2.39 (s, 3H); ${ }^{13} \mathrm{C}$ NMR (100 MHz, $\left.\mathrm{CDCl}_{3}\right): \delta 147.0,143.5,141.2,137.1,134.7,131.2,130.0,129.8,129.3,127.7$, 126.6, 125.2, 122.1, 121.4, 115.6, 65.8, 20.4; IR (ATR) $\mathrm{cm}^{-1}: 3054,3017,2954,2887,1607,1486,1456,1379,1353,1291$, 1247, 1218, 1176, 1127, 1114, 1077, 1017, 1030, 1014; ESI-HRMS m/z: $265.1235\left([\mathrm{M}+\mathrm{H}]^{+}\right)$; Calcd. for $\mathrm{C}_{18} \mathrm{H}_{17} \mathrm{O}_{2}$ : 265.1223 .

2'-(5-Methoxynaphthalen-2-yl)spiro[[1,3]dioxolane2,1'-indene] (2g)

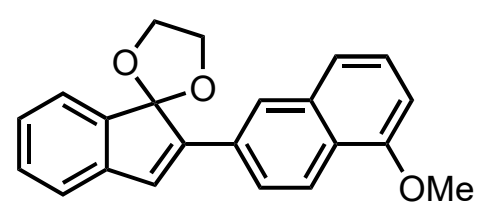

$2 \mathrm{~g}$

According to GP C, 2-(2-((6-methoxynaphthalen-2-yl)ethynyl)phenyl)-1,3-dioxolane (1g, $32.0 \mathrm{mg}, 0.1 \mathrm{mmol})$ and $\operatorname{IPrAuSbF}_{6}(4.1 \mathrm{mg}, 5.0 \mu \mathrm{mol})$ were dissolved in dichloroethane $(1 \mathrm{~mL})$ and stirred for $24 \mathrm{~h}$ at $80{ }^{\circ} \mathrm{C}$ in oil bath. Purification 
by flash silica-gel column chromatography (hexane / ethyl acetate $=10$ / 1 ) to give 2'-(5-methoxynaphthalen-2-yl)spiro[[1,3]dioxolane2,1'-indene] (2g, $19.5 \mathrm{mg}, 610.0 \mu \mathrm{mol})$ in $61 \%$ yield as a pale yellow solid.

MP: ${ }^{199-205}{ }^{\circ} \mathrm{C} ;{ }^{1} \mathrm{H}$ NMR $\left(400 \mathrm{MHz}, \mathrm{CDCl}_{3}\right): \delta 8.03(\mathrm{~s}, 1 \mathrm{H}), 7.76-7.70(\mathrm{~m}, 3 \mathrm{H}), 7.33(\mathrm{~d}, J=7.2 \mathrm{~Hz}, 1 \mathrm{H}), 7.28-7.24(\mathrm{~m}$, 1H), 7.19-7.12 (m, 4H), $6.96(\mathrm{~s}, 1 \mathrm{H}), 4.51-4.37$ (m, 4H), 3.93 (s, 3H); ${ }^{13} \mathrm{C} \mathrm{NMR}\left(100 \mathrm{MHz}, \mathrm{CDCl}_{3}\right): \delta 157.9,145.5,145.3$, $140.5,134.2,130.1,129.9,129.6,129.2,128.7,126.7,126.6,125.7,125.5,121.7,118.9,115.4,105.7,65.4,55.3,29.7 ;$ IR (ATR) $\mathrm{cm}^{-1}:$ 2956, 2931, 2895, 2349, 1737, 1721, 1709, 1658, 1620, 1603, 1563, 1536, 1511, 1501, 1478, 1467, 1452, 1391, 1339, 1314, 1288, 1264, 1218, 1203, 1181, 1164, 1119, 1082, 1025, 1000; ESI-HRMS m/z: $353.1171\left([\mathrm{M}+\mathrm{Na}]^{+}\right)$; Calcd. for $\mathrm{C}_{22} \mathrm{H}_{18} \mathrm{O}_{3} \mathrm{Na}: 353.1148$.

\section{2'-(Thiophen-3-yl)spiro[[1,3]dioxolane-2,1'-indene] (2h)}

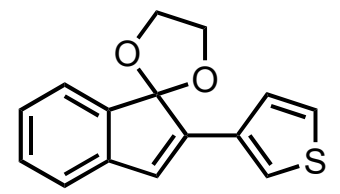

2h

According to GP C, 2-(2-(thiophen-3-ylethynyl)phenyl)-1,3-dioxolane (1h, $25.6 \mathrm{mg}, 0.1 \mathrm{mmol})$ and IPrAuSbF 6 (4.1 mg, $5.0 \mu \mathrm{mol})$ were dissolved in dichloroethane $(1 \mathrm{~mL})$ and stirred for $3 \mathrm{~h}$ at $80{ }^{\circ} \mathrm{C}$ in oil bath. Purification by flash silica-gel column chromatography (hexane / ethyl acetate $=15 / 1)$ to give 2'-(thiophen-3-yl)spiro[[1,3]dioxolane-2,1'-indene] $(\mathbf{2 h}$, $24.1 \mathrm{mg}, 940.0 \mu \mathrm{mol})$ in $94 \%$ yield as a brown solid.

MP: $100-101{ }^{\circ} \mathrm{C} ;{ }^{1} \mathrm{H}$ NMR $\left(400 \mathrm{MHz} \mathrm{CDCl}_{3}\right): \delta 7.47-7.46(\mathrm{~m}, 1 \mathrm{H}), 7.36-7.29(\mathrm{~m}, 3 \mathrm{H}), 7.23(\mathrm{~d}, J=7.2 \mathrm{~Hz}, 1 \mathrm{H})$, 7.16-7.12 (m, 2H), $6.82(\mathrm{~s}, 1 \mathrm{H}), 4.52-4.37(\mathrm{~m}, 4 \mathrm{H}) ;{ }^{13} \mathrm{C} \mathrm{NMR}\left(100 \mathrm{MHz}, \mathrm{CDCl}_{3}\right): \delta 145.0,140.8,140.5,134.2,129.6$, 129.1, 126.6, 126.6, 125.3, 122.2, 121.8, 121.7, 114.8, 65.3; IR (ATR) $\mathrm{cm}^{-1}:$ 2959, 2918, 2889, 2849, 1728, 1604, 1457, 1376, 1343, 1288, 1214, 1193, 1168, 1137, 1081, 1036; ESI-HRMS m/z: $257.0627\left([\mathrm{M}+\mathrm{H}]^{+}\right)$; Calcd. for $\mathrm{C}_{15} \mathrm{H}_{13} \mathrm{O}_{2} \mathrm{~S}$ : 257.0631.

2'-(4-Methoxyphenyl)-6'-methylspiro[[1,3]dioxolane-2,1'-indene] (2i)

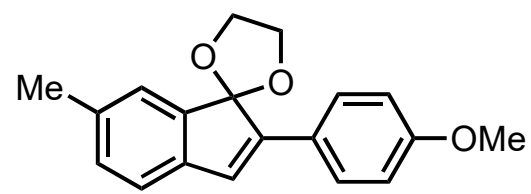

$2 \mathbf{i}$

According to GP C, 2-(2-((4-methoxyphenyl)ethynyl)-5-methylphenyl)-1,3-dioxolane (1i, $29.4 \mathrm{mg}, 0.1 \mathrm{mmol}), \mathrm{IPrAuCl}$ (3.1 mg, $5.0 \mu \mathrm{mol})$ and $\operatorname{AgSbF}_{6}(1.7 \mathrm{mg}, 5.0 \mu \mathrm{mol})$ were dissolved in dichloroethane $(1 \mathrm{~mL})$ and stirred for $6 \mathrm{~h}$ at $80{ }^{\circ} \mathrm{C}$ in oil bath. Purification by flash silica-gel column chromatography (hexane / ethyl acetate = 15/ 1) to give 2'-(4-methoxyphenyl)-6'-methylspiro[[1,3]dioxolane-2,1'-indene] (2i, $29.4 \mathrm{mg}, 0.1 \mathrm{mmol})$ in quantitative yield as a colorless solid.

MP: $174-175{ }^{\circ} \mathrm{C} ;{ }^{1} \mathrm{H}$ NMR $\left(400 \mathrm{MHz}, \mathrm{CDCl}_{3}\right): \delta 7.58(\mathrm{~d}, J=8.9 \mathrm{~Hz}, 2 \mathrm{H}), 7.11(\mathrm{~s}, 1 \mathrm{H}), 7.04-7.00(\mathrm{~m}, 2 \mathrm{H}), 6.89(\mathrm{~d}, J=8.9$ $\mathrm{Hz}, 2 \mathrm{H}), 6.75(\mathrm{~s}, 1 \mathrm{H}), 4.47-4.39(\mathrm{~m}, 2 \mathrm{H}), 4.36-4.28(\mathrm{~m}, 2 \mathrm{H}), 3.81(\mathrm{~s}, 3 \mathrm{H}), 2.33(\mathrm{~s}, 3 \mathrm{H}) ;{ }^{13} \mathrm{C} \mathrm{NMR}(100 \mathrm{MHz}, \mathrm{CDCl}): \delta$ 159.3, 145.5, 144.0, 137.8, 136.2, 129.8, 128.8, 128.0. 126.8, 122.8, 121.1, 115.3, 113.8, 65.3, 55.2, 21.5; IR (ATR) $\mathrm{cm}^{-1}$ : 2893, 2839, 2349, 1598, 1507, 1487, 1455, 1441, 1241, 1198, 1180, 1163, 1112, 1094, 1026, 1007; ESI-HRMS m/z: $317.1147\left([\mathrm{M}+\mathrm{Na}]^{+}\right)$; Calcd. for $\mathrm{C}_{19} \mathrm{H}_{18} \mathrm{O}_{3} \mathrm{Na}: 317.1148$. 


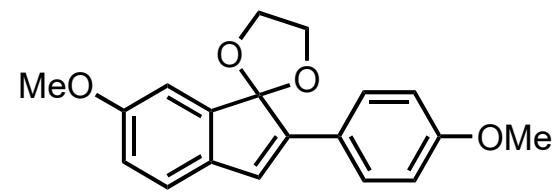

2j

According to GP C, 2-(5-methoxy-2-((4-methoxyphenyl)ethynyl)phenyl)-1,3-dioxolane (1j, $31.0 \mathrm{mg}, 0.1 \mathrm{mmol})$ and $\operatorname{IPrAuSbF}_{6}(4.1 \mathrm{mg}, 5.0 \mu \mathrm{mol})$ were dissolved in dichloroethane $(1 \mathrm{~mL})$ and stirred for $6 \mathrm{~h}$ at $80{ }^{\circ} \mathrm{C}$ in oil bath. Purification by flash silica-gel column chromatography (hexane / ethyl acetate = $5 /$ 1) to give 2'-(4-methoxyphenyl)-6'-methoxyspiro[[1,3]dioxolane-2,1'-indene] $(\mathbf{2} \mathbf{j}, 31.0 \mathrm{mg}, 0.1 \mathrm{mmol})$ in quantitative yield as a colorless solid.

MP: $157-158{ }^{\circ} \mathrm{C} ;{ }^{1} \mathrm{H}$ NMR (400 MHz, $\left.\mathrm{CDCl}_{3}\right): \delta 7.56(\mathrm{~d}, J=8.8 \mathrm{~Hz}, 2 \mathrm{H}), 7.04(\mathrm{~d}, J=8.0 \mathrm{~Hz}, 1 \mathrm{H}), 6.90-6.88(\mathrm{~m}, 3 \mathrm{H})$, 6.74-6.71 (m, 2H), 4.46-4.39 (m, 2H), 4.37-4.29 (m, 2H), $3.82(\mathrm{~s}, 3 \mathrm{H}), 3.81(\mathrm{~s}, 3 \mathrm{H}) ;{ }^{13} \mathrm{C} \mathrm{NMR}\left(100 \mathrm{MHz}, \mathrm{CDCl}_{3}\right): \delta 159.2$, $158.9,147.2,134.1,133.2,128.7,127.9,126.8,121.8,115.1,113.8,112.5,110.3,65.3,55.6,55.2$; IR (ATR) $\mathrm{cm}^{-1}: 2960$, 2920, 2898, 2850, 2037, 1737, 1605, 1580, 1504, 1478, 1449, 1418, 1346, 1281, 1249, 1200, 1179, 1167, 1112, 1024, 1001; ESI-HRMS m/z: $311.1274\left([\mathrm{M}+\mathrm{H}]^{+}\right)$; Calcd. for $\mathrm{C}_{19} \mathrm{H}_{19} \mathrm{O}_{4}: 311.1278$.

2'-(4-Methoxyphenyl)-6'-nitrospiro[[1,3]dioxolane-2,1'-indene] (2k)

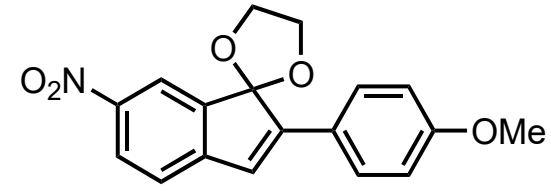

2k

According to GP C, 2-(2-((4-methoxyphenyl)ethynyl)-5-nitrophenyl)-1,3-dioxolane (1k, $32.5 \mathrm{mg}, 0.1 \mathrm{mmol}), \operatorname{IPrAuCl}(3.1$ $\mathrm{mg}, 5.0 \mu \mathrm{mol})$ and $\mathrm{AgSbF}_{6}(1.7 \mathrm{mg}, 5.0 \mu \mathrm{mol})$ were dissolved in dichloroethane $(1 \mathrm{~mL})$ and stirred for $6 \mathrm{~h}$ at $80{ }^{\circ} \mathrm{C}$ in oil bath. Purification by flash silica-gel column chromatography (hexane / ethyl acetate $=5 / 1$ ) to give 2'-(4-methoxyphenyl)-6'-nitrospiro[[1,3]dioxolane-2,1'-indene] (2k, $30.6 \mathrm{mg}, 940.0 \mu \mathrm{mol})$ in 94\% yield as a yellow solid. MP: $165-167{ }^{\circ} \mathrm{C} ;{ }^{1} \mathrm{H}$ NMR $\left(400 \mathrm{MHz}, \mathrm{CDCl}_{3}\right): \delta 8.19(\mathrm{dd}, J=1.9,8.1 \mathrm{~Hz}, 1 \mathrm{H}), 8.08(\mathrm{~d}, J=1.9 \mathrm{~Hz}, 1 \mathrm{H}), 7.61(\mathrm{~d}, J=7.8$ $\mathrm{Hz}, 2 \mathrm{H}), 7.23(\mathrm{~d}, J=8.1 \mathrm{~Hz}, 1 \mathrm{H}), 6.93(\mathrm{~d}, J=7.8 \mathrm{~Hz}, 2 \mathrm{H}), 6.84(\mathrm{~s}, 1 \mathrm{H}), 4.55-4.47(\mathrm{~m}, 2 \mathrm{H}), 4.45-4.37(\mathrm{~m}, 2 \mathrm{H}), 3.85(\mathrm{~s}$, $3 \mathrm{H}) ;{ }^{13} \mathrm{C}$ NMR (100 MHz, $\left.\mathrm{CDCl}_{3}\right): \delta 160.4,150.7,147.6,146.4,146.3,128.5,126.7,126.3,125.4,121.1,116.8,114.1$, 114.0, 65.7, 55.3; IR (ATR) $\mathrm{cm}^{-1}:$ 3073, 2986, 2910, 2841, 1593, 1563, 1501, 1459, 1440, 1419, 1331, 1291, 1269, 1250 , 1215, 1188, 1173, 1137, 1111, 1068, 1035; Anal. Calcd. for $\mathrm{C}_{18} \mathrm{H}_{15} \mathrm{NO}_{5}$ : C, 66.46; H, 4.65; N, 4.31. Found: C, 66.61; H, $4.63 ; \mathrm{N}, 4.52$.

2'-(4-(2-Methyl-1,3-dioxolan-2-yl)phenyl)spiro[[1,3]dioxolane2,1'-indene] (2l)

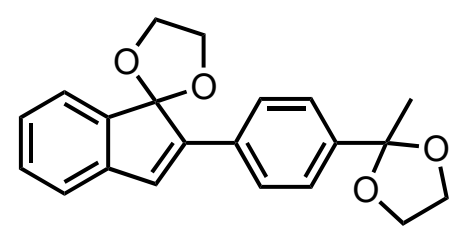

2)

According to GP C, 2-(4-((2-(1,3-dioxolan-2-yl)phenyl)ethynyl)phenyl)-2-methyl-1,3-dioxolane (11, $33.6 \mathrm{mg}, 0.1 \mathrm{mmol})$, $\mathrm{MgSO}_{4}(16.8 \mathrm{mg}, 0.1 \mathrm{mmol})$ and $\mathrm{IPrAuSbF}_{6}(4.1 \mathrm{mg}, 5.0 \mu \mathrm{mol})$ were dissolved in dichloroethane $(1 \mathrm{~mL})$ and stirred for $3 \mathrm{~h}$ at $80{ }^{\circ} \mathrm{C}$ in oil bath. Purification by flash silica-gel column chromatography (hexane / ethyl acetate $=5 / 1$ ) to give 
2'-(4-(2-methyl-1,3-dioxolan-2-yl)phenyl)spiro[[1,3]dioxolane2,1'-indene] (2l, $28.1 \mathrm{mg}, 840.0 \mu \mathrm{mol})$ in $84 \%$ yield as a colorless solid.

MP: $174-175^{\circ} \mathrm{C} ;{ }^{1} \mathrm{H}$ NMR $\left(400 \mathrm{MHz}, \mathrm{CDCl}_{3}\right): \delta 7.63(\mathrm{~d}, J=8.6 \mathrm{~Hz}, 2 \mathrm{H}), 7.47(\mathrm{~d}, J=8.6 \mathrm{~Hz}, 2 \mathrm{H}), 7.30(\mathrm{~d}, J=7.2 \mathrm{~Hz}, 1 \mathrm{H})$, 7.26-7.23 (m, 1H), 7.17-7.13 (m, 2H), $6.90(\mathrm{~s}, 1 \mathrm{H}), 4.49-4.32(\mathrm{~m}, 4 \mathrm{H}), 4.09-4.00(\mathrm{~m}, 2 \mathrm{H}), 3.84-3.75(\mathrm{~m}, 2 \mathrm{H}), 1.67(\mathrm{~s}$, $3 \mathrm{H}) ;{ }^{13} \mathrm{C}$ NMR (100 MHz, $\left.\mathrm{CDCl}_{3}\right): \delta 145.5,144.7,143.0,140.2,133.4,130.5,129.6,126.8,126.6,125.3,121.8,121.6$, 115.3, 108.7, 65.3, 64.4, 27.5; IR (ATR) $\mathrm{cm}^{-1}: 2987,2962,2899,2349,1599,1505,1469,1455,1407,1370,1352,1322$, 1298, 1254, 1218, 1205, 1192, 1144, 1098, 1084, 1030, 1014; ESI-HRMS m/z: $337.1440\left([\mathrm{M}+\mathrm{H}]^{+}\right)$; Calcd. for $\mathrm{C}_{21} \mathrm{H}_{21} \mathrm{O}_{4}$ : 337.1434 .

\section{2'-(Cyclohex-1-en-1-yl)spiro[[1,3]dioxolane-2,1'-indene] (2m)}

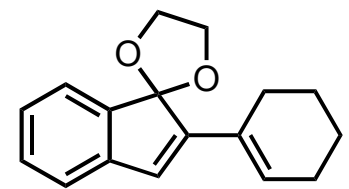

$2 \mathrm{~m}$

According to GP C, 2-(2-(cyclohex-1-enethynyl)phenyl-1,3-dioxolane (1m, $25.4 \mathbf{m g}, 0.1 \mathrm{mmol})$ and $\operatorname{IPrAuSbF}_{6}(4.1 \mathrm{mg}$, $5.0 \mu \mathrm{mol})$ were dissolved in dichloroethane $(1 \mathrm{~mL})$ and stirred for $12 \mathrm{~h}$ at $80{ }^{\circ} \mathrm{C}$ in oil bath. Purification by flash silica-gel column chromatography (hexane / ethyl acetate $=20 / 1$ ) to give 2'-(cyclohex-1-en-1-yl)spiro[[1,3]dioxolane-2,1'-indene] ( $\mathbf{2 m}, 22.9 \mathrm{mg}, 900.0 \mu \mathrm{mol})$ in $90 \%$ yield as a yellow oil.

${ }^{1} \mathrm{H}$ NMR (400 MHz, $\left.\mathrm{CDCl}_{3}\right): \delta 7.25-7.18(\mathrm{~m}, 2 \mathrm{H}), 7.10-7.06(\mathrm{~m}, 2 \mathrm{H}), 6.53(\mathrm{~s}, 1 \mathrm{H}), 6.16(\mathrm{dd}, J=8.0,8.0 \mathrm{~Hz}, 1 \mathrm{H})$, $4.51-4.35(\mathrm{~m}, 4 \mathrm{H}), 2.32-2.23(\mathrm{~m}, 4 \mathrm{H}), 1.77-1.72(\mathrm{~m}, 2 \mathrm{H}), 1.68-1.61(\mathrm{~m}, 2 \mathrm{H}) ;{ }^{13} \mathrm{C} \mathrm{NMR}\left(100 \mathrm{MHz}, \mathrm{CDCl}_{3}\right): \delta 146.0,145.1$, 140.3, 129.6, 129.3, 128.0, 127.6, 126.1, 121.5, 121.3, 114.9, 64.8, 26.3, 26.1, 22.6, 21.9; IR (ATR) $\mathrm{cm}^{-1}: 2924,2857,1710$, 1604, 1454, 1383, 1349, 1318, 1283, 1233, 1207, 1175, 1136, 1082, 1955, 1016; ESI-HRMS m/z: 277.1193 ([M+Na] $)$; Calcd. for $\mathrm{C}_{17} \mathrm{H}_{18} \mathrm{O}_{2} \mathrm{Na}$ : 277.1199.

2'-Hexylspiro[[1,3]dioxolane-2,1'-indene] (2n)

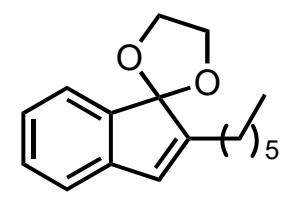

2n

According to GP C, 2-(2-oct-1-yn-1lethynyl)phenyl)-1,3-dioxolane (1n, $25.8 \mathrm{mg}, 0.1 \mathrm{mmol})$ and $\mathrm{IPrAuSbF}_{6}(4.1 \mathrm{mg}, 5.0$ $\mu \mathrm{mol})$ were dissolved in dichloroethane $(1 \mathrm{~mL})$ and stirred for $6 \mathrm{~h}$ at $80{ }^{\circ} \mathrm{C}$ in oil bath. Purification by flash silica-gel column chromatography (hexane / ethyl acetate $=20 / 1)$ to give 2'-hexylspiro[[1,3]dioxolane-2,1'-indene] $(\mathbf{2 n}, 25.8 \mathrm{mg}$, $0.1 \mathrm{mmol})$ in quantitative yiled as a yellow oil.

${ }^{1} \mathrm{H}$ NMR (400 MHz, $\left.\mathrm{CDCl}_{3}\right): \delta$ 7.25-7.15 (m, 2H), 7.07-7.00 (m, 2H), $6.26(\mathrm{~s}, 1 \mathrm{H}), 4.38-4.29(\mathrm{~m}, 2 \mathrm{H}), 4.27-4.19(\mathrm{~m}, 2 \mathrm{H})$, $2.19(\mathrm{t}, J=6.6 \mathrm{~Hz}, 2 \mathrm{H}), 1.65-1.57(\mathrm{~m}, 2 \mathrm{H}), 1.41-1.30(\mathrm{~m}, 6 \mathrm{H}), 0.90(\mathrm{t}, J=6.6 \mathrm{~Hz}, 3 \mathrm{H}) ;{ }^{13} \mathrm{C} \mathrm{NMR}\left(100 \mathrm{MHz}, \mathrm{CDCl}_{3}\right): \delta$ 150.0, 144.6, 141.7, 129.5, 127.1, 125.6, 121.6, 120.7, 114.4, 65.9, 31.7, 29.3, 27.0, 25.7, 22.6, 14.1; IR (ATR) $\mathrm{cm}^{-1}: 2955$, 2925, 2857, 1608, 1458, 1291, 1240, 1218, 1158, 1113, 1078, 1009; ESI-HRMS m/z: $259.1699\left([\mathrm{M}+\mathrm{H}]^{+}\right)$; Calcd. for $\mathrm{C}_{17} \mathrm{H}_{23} \mathrm{O}_{2}: 259.1693$. 


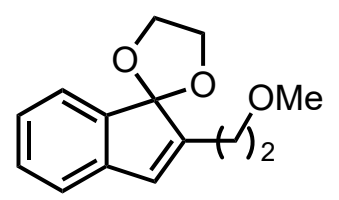

20

According to GP C, 2-(2-(4-methoxybut-1-yn-1-yl)phenyl)-1,3-dioxolane (1o, $23.2 \mathrm{mg}, 0.1 \mathrm{mmol})$ and $\operatorname{IPrAuSbF}_{6}(4.1 \mathrm{mg}$, $5.0 \mu \mathrm{mol})$ were dissolved in dichloroethane $(1 \mathrm{~mL})$ and stirred for $6 \mathrm{~h}$ at $80^{\circ} \mathrm{C}$ in oil bath. Purification by flash silica-gel column chromatography (hexane / ethyl acetate $=20 / 1)$ to give 2'-(2-methoxyethyl)spiro[[1,3]dioxolane-2,1'-indene] $(\mathbf{2 o}$, $22.3 \mathrm{mg}, 960.0 \mu \mathrm{mol})$ in quantitative yield as a colorless oil.

${ }^{1} \mathrm{H}$ NMR $\left(400 \mathrm{MHz}, \mathrm{CDCl}_{3}\right): \delta$ 7.23-7.16 (m ,2H), 7.12-7.01 (m, 2H), $6.36(\mathrm{~s}, 1 \mathrm{H}), 4.38-4.30(\mathrm{~m}, 2 \mathrm{H}), 4.28-4.22(\mathrm{~m}, 2 \mathrm{H})$, $3.65(\mathrm{t}, J=7.0 \mathrm{~Hz}, 2 \mathrm{H}), 3.39$ (s, 3H), $2.52(\mathrm{t}, J=7.0,2 \mathrm{H}) ;{ }^{13} \mathrm{C}$ NMR $\left(100 \mathrm{MHz}, \mathrm{CDCl}_{3}\right): \delta 145.8,144.4,141.3,129.5$, 128.7, 125.9, 121.6, 120.9, 114.4, 70.8, 65.9, 58.6, 26.1; IR (ATR) $\mathrm{cm}^{-1}: 2889,1608,1457,1384,1239,1192,1158,1111$, 1078, 1008; ESI-HRMS m/z: $255.0984\left([\mathrm{M}+\mathrm{Na}]^{+}\right)$) Calcd. for $\mathrm{C}_{14} \mathrm{H}_{16} \mathrm{O}_{3} \mathrm{Na}: 255.0992$.

2'-(4-Methoxyphenyl)4',5',6',7'-tetrahydrospiro[[1,3]dioxolan-2,1'-indene] (2p)

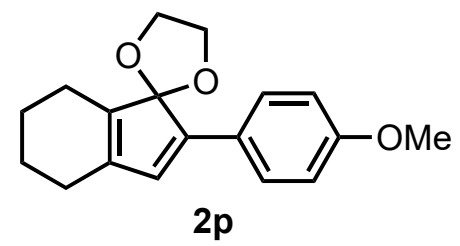

According to GP C, 2-(4-methoxybut-1-yn-1-yl)benzaldehyde (1o, $23.2 \mathrm{mg}, 0.1 \mathrm{mmol})$ and $\operatorname{IPrAuSbF}_{6}(4.1 \mathrm{mg}, 5.0 \mu \mathrm{mol})$ were dissolved in dichloroethane $(1 \mathrm{~mL})$ and stirred for $6 \mathrm{~h}$ at $80{ }^{\circ} \mathrm{C}$ in oil bath. Purification by flash silica-gel column chromatography (hexane $/$ ethyl acetate $=20$ / 1 ) to give 2'-(4-methoxyphenyl)4',5',6',7'-tetrahydrospiro[[1,3]dioxolan-2,1'-indene] (2p, $22.3 \mathrm{mg}, 960.0 \mu \mathrm{mol})$ in $74 \%$ yield as a yellow oil.

${ }^{1} \mathrm{H}$ NMR (500 MHz, CDCl $): \delta 7.44(\mathrm{~d}, J=8.5 \mathrm{~Hz}, 2 \mathrm{H}), 6.85(\mathrm{~d}, J=8.5 \mathrm{~Hz}, 2 \mathrm{H}), 6.16(\mathrm{~s}, 1 \mathrm{H}), 4.24-4.18(\mathrm{~m}, 4 \mathrm{H}), 3.80(\mathrm{~s}$, 3H), 2.18-2.14 (m, 4H), 1.71-1.66 (m, 4H); ${ }^{13} \mathrm{C} \mathrm{NMR}\left(100 \mathrm{MHz}, \mathrm{CDCl}_{3}\right): \delta 159.4,139.7,132.8,122.3,115.6,113.8,103.2$, 93.1, 86.4, 65.6, 55.2, 30.7, 22.2, 21.9, 21.7; IR (ATR) $\mathrm{cm}^{-1}: 2930,2882,2837,2198,1669,1603,1568,1507,1461,1439$, 1387, 1284, 1245, 1221, 1172, 1127, 1105, 1091, 1054, 1027; ESI-HRMS m/z: $285.1486\left([\mathrm{M}+\mathrm{H}]^{\dagger}\right)$; Calcd. for $\mathrm{C}_{18} \mathrm{H}_{21} \mathrm{O}_{3}$ : 285.1485 .

2'-(4-Methoxyphenyl)spiro[[1,3]dioxane-2,1'indene] (2q)

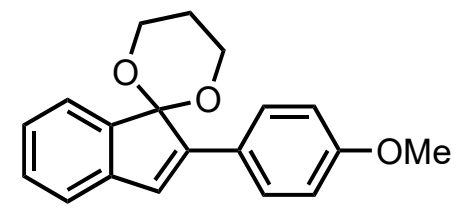

$2 q$

According to GP C, 2-(2-((4-methoxyphenyl)ethynyl)phenyl)-1,3-dioxane (1q, $29.4 \mathrm{mg}, 0.1 \mathrm{mmol})$ and $\mathrm{IPAuSbF}_{6}(4.1 \mathrm{mg}$, $5.0 \mu \mathrm{mol})$ were dissolved in dichloroethane $(1 \mathrm{~mL})$ and stirred for $6 \mathrm{~h}$ at $80{ }^{\circ} \mathrm{C}$ in oil bath. Purification by flash silica-gel column chromatography (hexane / ethyl acetate $=10 / 1)$ to give 2'-(4-methoxyphenyl)spiro[[1,3]dioxane-2,1'indene] (2q, $27.1 \mathrm{mg}, 920.0 \mu \mathrm{mol})$ in $92 \%$ yield as a orangish solid.

MP: $113-115{ }^{\circ} \mathrm{C} ;{ }^{1} \mathrm{H}$ NMR (400 MHz, $\left.\mathrm{CDCl}_{3}\right): \delta 7.93$ (d, $\left.J=8.8 \mathrm{~Hz}, 2 \mathrm{H}\right), 7.83(\mathrm{~d}, J=7.6 \mathrm{~Hz}, 1 \mathrm{H}), 7.30-7.20(\mathrm{~m}, 2 \mathrm{H}), 7.12$ (dd, $J=7.6,7.6 \mathrm{~Hz}, 1 \mathrm{H}), 6.92(\mathrm{~d}, J=8.8 \mathrm{~Hz}, 2 \mathrm{H}), 6.84(\mathrm{~s}, 1 \mathrm{H}), 4.71(\mathrm{dd}, J=12.8,12.8 \mathrm{~Hz}, 2 \mathrm{H}), 4.15(\mathrm{dd}, J=12.8,5.2 \mathrm{~Hz}$, 
2H), $3.82(\mathrm{~s}, 3 \mathrm{H}), 2.64-2.52(\mathrm{~m}, 1 \mathrm{H}), 1.74-1.71(\mathrm{~m}, 1 \mathrm{H}) ;{ }^{13} \mathrm{C} \mathrm{NMR}\left(100 \mathrm{MHz}, \mathrm{CDCl}_{3}\right): \delta 159.4,146.0,142.7,141.8,129.2$, $128.5,125.7,125.5,125.0,124.4,122.1,113.7,106.9,61.3,55.3,25.3$; IR (ATR) $\mathrm{cm}^{-1}: 2959,2887,2837,1722,1599,1509$, 1481, 1459, 1363, 1301, 1277, 1249, 1203, 1177, 1134, 1113, 1087, 1065, 1030, 1004; ESI-HRMS m/z: 317.1162 $\left([\mathrm{M}+\mathrm{Na}]^{+}\right)$; Calcd. for $\mathrm{C}_{19} \mathrm{H}_{18} \mathrm{O}_{3} \mathrm{Na}: 317.1148$.

\section{2'-(4-Methoxyphenyl)-5,5-dimethylspiro[[1,3]dioxane-2,1'-indene] (2r)}

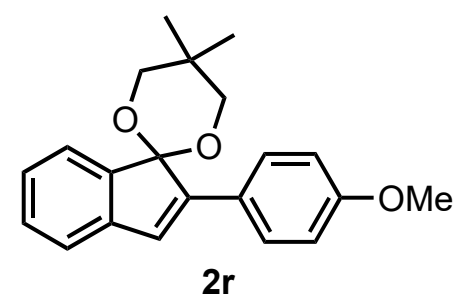

According to GP C, 2-(2-((4-methoxyphenyl)ethynyl)phenyl)-5,5-dimethyl-1,3-dioxane (1r, $16.1 \mathrm{mg}, 50.0 \mu \mathrm{mol})$ and $\operatorname{IPrAuSbF}_{6}(2.1 \mathrm{mg}, 2.5 \mu \mathrm{mol})$ were dissolved in dichloroethane $(0.5 \mathrm{~mL})$ and stirred for $6 \mathrm{~h}$ at $80{ }^{\circ} \mathrm{C}$ in oil bath. Purification by flash silica-gel column chromatography (hexane / ethyl acetate $=10 /$ ) to give 2'-(4-methoxyphenyl)-5,5-dimethylspiro[[1,3]dioxane-2,1'-indene] (2r, $16.1 \mathrm{mg}, 50.0 \mu \mathrm{mol})$ in quantitative yield as a colorless solid.

MP: $140-141^{\circ} \mathrm{C} ;{ }^{1} \mathrm{H}$ NMR $\left(400 \mathrm{MHz}, \mathrm{CDCl}_{3}\right): \delta 7.89(\mathrm{~d}, J=8.6 \mathrm{~Hz}, 2 \mathrm{H}), 7.69(\mathrm{~d}, J=7.2 \mathrm{~Hz}, 1 \mathrm{H}), 7.28(\mathrm{dd}, J=7.2,7.2$ $\mathrm{Hz}, 1 \mathrm{H}), 7.21(\mathrm{~d}, J=7.2 \mathrm{~Hz}, 1 \mathrm{H}), 7.11(\mathrm{ddd}, J=0.8,7.2,7.2 \mathrm{~Hz}, 1 \mathrm{H}), 6.92(\mathrm{~d}, J=8.6 \mathrm{~Hz}, 2 \mathrm{H}), 6.75(\mathrm{~s}, 1 \mathrm{H}), 4.43(\mathrm{~d}, J=$ $12.2 \mathrm{~Hz}, 2 \mathrm{H}), 3.83(\mathrm{~s}, 3 \mathrm{H}), 3.69(\mathrm{~d}, J=12.2 \mathrm{~Hz}, 2 \mathrm{H}), 1.46(\mathrm{~s}, 3 \mathrm{H}), 0.97(\mathrm{~s}, 3 \mathrm{H}) ;{ }^{13} \mathrm{C} \mathrm{NMR}\left(100 \mathrm{MHz}, \mathrm{CDCl}_{3}\right): \delta 159.5$, 146.9, 142.0, 141.8, 129.3, 128.7, 127.5, 126.7, 125.1, 124.8, 122.1, 113.6, 107.2, 71.8, 55.2, 30.1, 25.0, 23.0; IR (ATR) $\mathrm{cm}^{-1}: 2980,2948,2925,2873,2836,1598,1509,1456,1393,1334,1296,1276,1253,1201,1170,1138,1111,1087,1071$, 1020,; ESI-HRMS m/z: $323.1644\left([\mathrm{M}+\mathrm{H}]^{+}\right)$; Calcd. for $\mathrm{C}_{21} \mathrm{H}_{23} \mathrm{O}_{3}: 323.1642$.

\section{1,3-Dimethoxy-2-(4-methoxyphenyl)-1H-indene (5)}

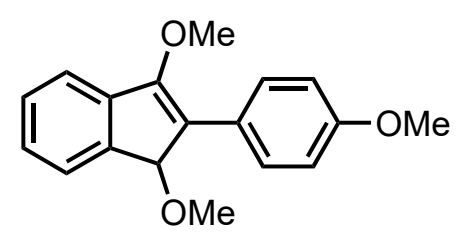

5

According to GP C, 1-(dimethoxymethyl)-2-((4-methoxyphenyl)ethynyl)benzene (1t, $28.2 \mathrm{mg}, 0.1 \mathrm{mmol}), \operatorname{IPrAuCl}(3.1 \mathrm{mg}$, $5.0 \mu \mathrm{mol})$ and $\mathrm{AgSbF}_{6}(1.7 \mathrm{mg}, 5.0 \mu \mathrm{mol})$ were dissolved in dichloroethane $(1 \mathrm{~mL})$ and stirred for $6 \mathrm{~h}$ at $80{ }^{\circ} \mathrm{C}$ in oil bath. Purification by flash silica-gel column chromatography (hexane / ethyl acetate $=15 / 1$ ) to give 1,3-dimethoxy-2-(4-methoxyphenyl)-1 $H$-indene $(22.8 \mathrm{mg}, 810.0 \mu \mathrm{mol})$ in $81 \%$ yield as a orangish oil.

${ }^{1} \mathrm{H}$ NMR (400 MHz, $\left.\mathrm{CDCl}_{3}\right): \delta 7.49(\mathrm{~d}, J=8.8 \mathrm{~Hz}, 2 \mathrm{H}), 7.38(\mathrm{~d}, J=7.0 \mathrm{~Hz}, 1 \mathrm{H}), 7.22(\mathrm{~d}, J=7.0 \mathrm{~Hz}, 1 \mathrm{H}), 7.16-7.09$ (m, $2 \mathrm{H}), 6.97(\mathrm{~d}, J=8.8 \mathrm{~Hz}, 2 \mathrm{H}), 5.28(\mathrm{~s}, 1 \mathrm{H}), 3.94(\mathrm{~s}, 3 \mathrm{H}), 3.85(\mathrm{~s}, 3 \mathrm{H}), 3.20(\mathrm{~s}, 3 \mathrm{H}) ;{ }^{13} \mathrm{C} \mathrm{NMR}\left(125 \mathrm{MHz}, \mathrm{CDCl}_{3}\right): \delta 158.6$, $157.3,144.1,135.7,130.0,128.7,125.3,123.9,123.6,118.5,118.3,113.7,79.0,58.4,55.3,51.9$; IR (ATR) $\mathrm{cm}^{-1}: 2936$, 2835, 1736, 1627, 1604, 1510, 1459, 1339, 1283, 1242, 1175, 1157, 1104, 1075, 1045, 1032; ESI-HRMS m/z: 305.1127 $\left([\mathrm{M}+\mathrm{Na}]^{+}\right)$; Calcd. for $\mathrm{C}_{18} \mathrm{H}_{18} \mathrm{O}_{3} \mathrm{Na}: 305.1148$.

2'-(4-Methoxyphenyl)-6'-methoxyspiro[[1,3]dioxolane-2,1'-indene]- $d_{1}\left(2 j-d_{1}\right)$ 


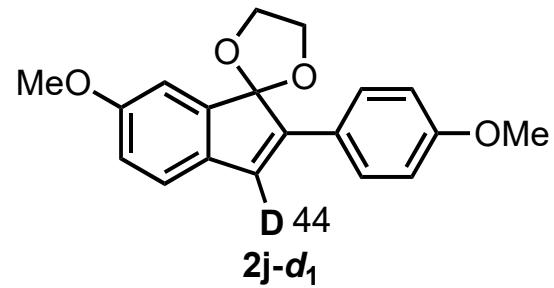

According to GP C, 2-(5-methoxy-2-((4-methoxyphenyl)ethynyl)phenyl)-1,3-dioxolane- $d_{1}\left(\mathbf{1} \mathbf{j}-\mathbf{d}_{\mathbf{1}}, 31.1 \mathrm{mg}, 0.1 \mathrm{mmol}\right)$ and $\mathrm{IPrAuSbF}_{6}(4.1 \mathrm{mg}, 5.0 \mu \mathrm{mol})$ were dissolved in dichloroethane $(1 \mathrm{~mL})$ and stirred for $6 \mathrm{~h}$ at $80{ }^{\circ} \mathrm{C}$ in oil bath. Purification by flash silica-gel column chromatography (hexane / ethyl acetate = 5 / 1) to give 2'-(4-methoxyphenyl)-6'-methoxyspiro[[1,3]dioxolane-2,1'-indene]- $d_{1}\left(\mathbf{2} \mathbf{j}-\mathbf{d}_{\mathbf{1}}, 24.9 \mathrm{mg}, 800.0 \mu \mathrm{mol}\right)$ in $80 \%$ yield as a colorless solid.

${ }^{1} \mathrm{H}$ NMR $\left(400 \mathrm{MHz}, \mathrm{CDCl}_{3}\right): \delta 7.56(\mathrm{~d}, J=8.8 \mathrm{~Hz}, 2 \mathrm{H}), 7.03(\mathrm{~d}, J=8.4 \mathrm{~Hz}, 1 \mathrm{H}), 6.90-6.87(\mathrm{~m}, 3 \mathrm{H}), 6.74-6.71(\mathrm{~m}, 1.54 \mathrm{H})$, 4.46-4.38 (m, 2H), 4.36-4.28 (m, 2H), $3.81(\mathrm{~s}, 3 \mathrm{H}), 3.80(\mathrm{~s}, 3 \mathrm{H}) ;{ }^{2} \mathrm{H}$ NMR $\left(77 \mathrm{MHz}, \mathrm{CHCl}_{3}\right): \delta 6.80$ (brs)

\section{2'-(4-Methoxyphenyl)spiro[[1,3]dioxolane-2,1'-indene $]-d_{1}\left(2 a-d_{1}\right)$}

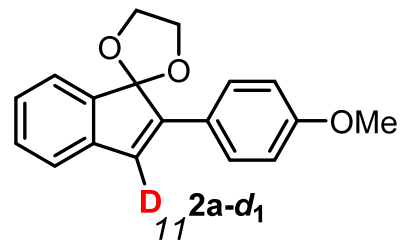

According to GP C, 2-(2-((4-methoxyphenyl)ethynyl)phenyl)-1,3-dioxolane (1a, $56.0 \mathrm{mg}, 0.2 \mathrm{mmol}), \mathrm{D}_{2} \mathrm{O}(1.0 \mu \mathrm{L}, 60.0$ $\mu \mathrm{mol})$ and $\operatorname{IPrAuSbF}_{6}(8.2 \mathrm{mg}, 10.0 \mu \mathrm{mol})$ were dissolved in dichloroethane $(2 \mathrm{~mL})$ and stirred for $6 \mathrm{~h}$ at $80{ }^{\circ} \mathrm{C}$ in oil bath. Purification by flash silica-gel column chromatography (hexane / ethyl acetate = 20 / 1) to give 2'-(4-methoxyphenyl)spiro[[1,3]dioxolane-2,1'-indene]- $d_{1}\left(\mathbf{2 a - d _ { 1 }}, 39.6 \mathrm{mg}, 140.0 \mu \mathrm{mol}\right)$ in $70 \%$ yield as a pale yellow solid.

${ }^{1} \mathrm{H}$ NMR $\left(400 \mathrm{MHz}, \mathrm{CDCl}_{3}\right): \delta 7.59(\mathrm{~d}, J=8.8 \mathrm{~Hz}, 2 \mathrm{H}), 7.28(\mathrm{~d}, J=7.6 \mathrm{~Hz}, 1 \mathrm{H}), 7.22(\mathrm{dd}, J=6.6,6.6 \mathrm{~Hz}, 1 \mathrm{H}), 7.13-7.09$ $(\mathrm{m}, 2 \mathrm{H}), 6.90(\mathrm{~d}, J=8.8 \mathrm{~Hz}, 2 \mathrm{H}), 6.78(\mathrm{~s}, 0.89 \mathrm{H}), 4.46-4.38(\mathrm{~m}, 2 \mathrm{H}), 4.36-4.28(\mathrm{~m}, 2 \mathrm{H}), 3.81(\mathrm{~s}, 3 \mathrm{H}) ;{ }^{2} \mathrm{H} \mathrm{NMR}(77 \mathrm{MHz}$, $\left.\mathrm{CHCl}_{3}\right): \delta 6.85$ (brs)

2',6'-Bis(4-methoxyphenyl)spiro[bis[1,3]dioxolane-2,1',6,1',-s-indacene] (11)

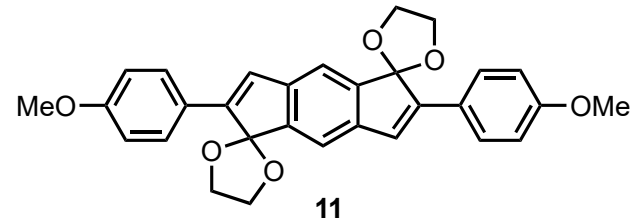

According to GP C, 2,2'-(2,5-bis((4-methoxyphenyl)ethynyl)-1,4-phenylene)bis(1,3-dioxolane) (10, $24.1 \mathrm{mg}, 50.0 \mu \mathrm{mol})$ and $\operatorname{IPrAuSbF}_{6}(2.1 \mathrm{mg}, 2.5 \mu \mathrm{mol})$ were dissolved in dichloroethane $(2 \mathrm{~mL})$ and stirred for $6 \mathrm{~h}$ at $80{ }^{\circ} \mathrm{C}$ in oil bath. Purification by crystallization with $\mathrm{CH}_{2} \mathrm{Cl}_{2}$ and hexane to 2',6'-bis(4-methoxyphenyl)spiro[bis[1,3]dioxolane-2,1',6,1'"-s-indacene] (11, $21.1 \mathrm{mg}, 42.0 \mu \mathrm{mol})$ in $87 \%$ yield as a yellow solid.

MP: $138-139{ }^{\circ} \mathrm{C} ;{ }^{1} \mathrm{H}$ NMR $\left(400 \mathrm{MHz}, \mathrm{CDCl}_{3}\right): \delta 7.58(\mathrm{~d}, J=8.4 \mathrm{~Hz}, 4 \mathrm{H}), 7.09(\mathrm{~s}, 2 \mathrm{H}), 6.90(\mathrm{~d}, J=8.4 \mathrm{~Hz}, 4 \mathrm{H}), 6.76(\mathrm{~s}$, 2H), 4.48-4.34 (m, 8H), 3.83 (s, 6H); IR (ATR) $\mathrm{cm}^{-1}: 2921,2836,1687,1597,1573,1509,1459,1412,1245,1168,1108$, 1078, 1024; ESI-HRMS m/z: $483.1809\left([\mathrm{M}+\mathrm{H}]^{+}\right)$; Calcd. for $\mathrm{C}_{30} \mathrm{H}_{27} \mathrm{O}_{6}: 483.1802$. 


\section{HMBC and NOESY NMR spectra}

The chemical structure of products (2) were elucidated based on various instrumental data, and were determined by HMBC and NOESY NMR experiments using $\mathbf{2 k}$ as a representative model.

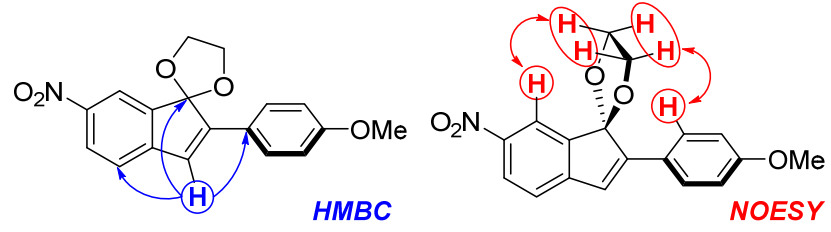

HMBC NMR (400 MHz, $\mathrm{CDCl}_{3}$ ) of 2'-(4-methoxyphenyl)-6'-nitrospiro[[1,3]dioxolane-2,1'-indene] (2k)

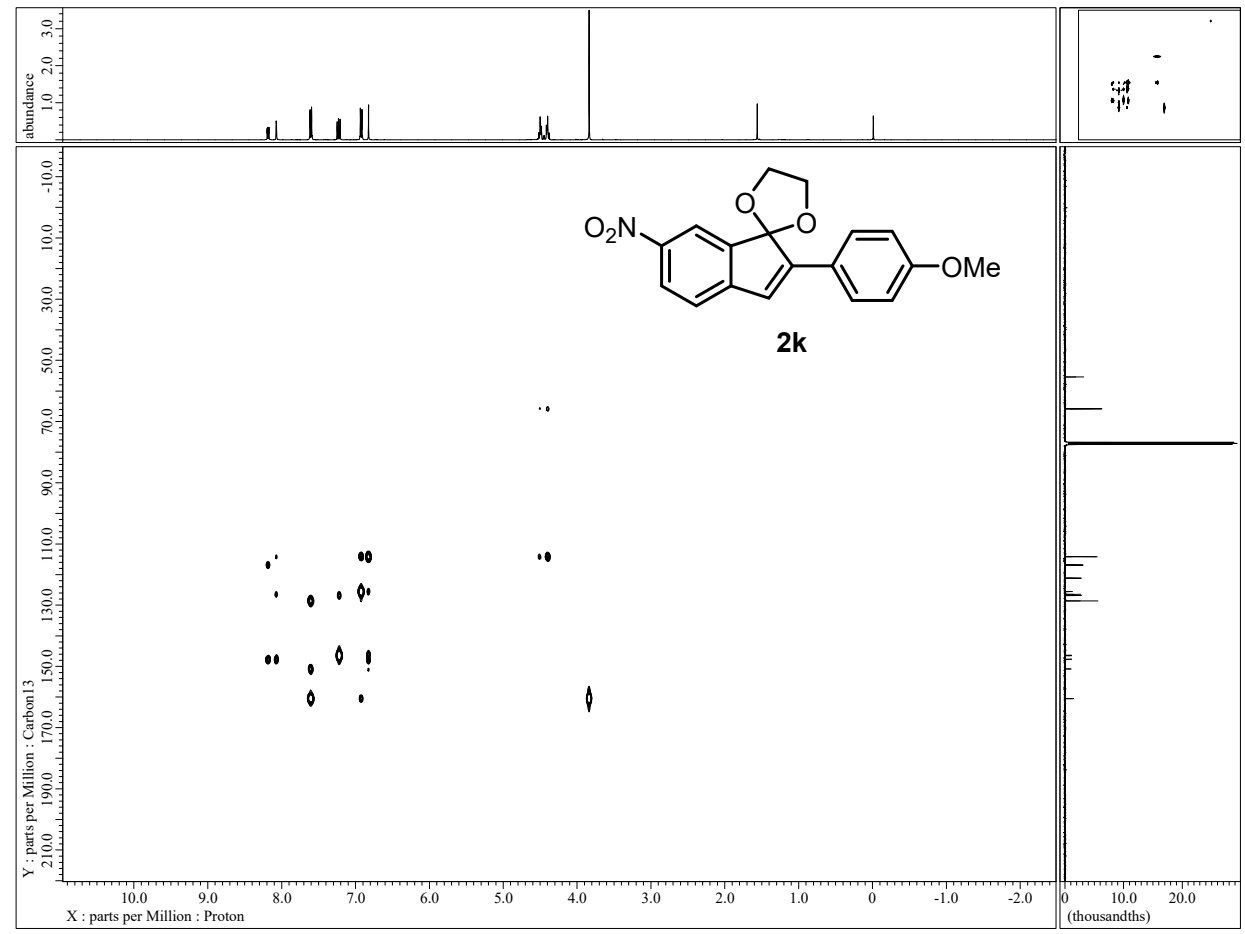

NOESY NMR (400 MHz, $\mathrm{CDCl}_{3}$ ) of 2'-(4-methoxyphenyl)-6'-nitrospiro[[1,3]dioxolane-2,1'-indene] (2k)

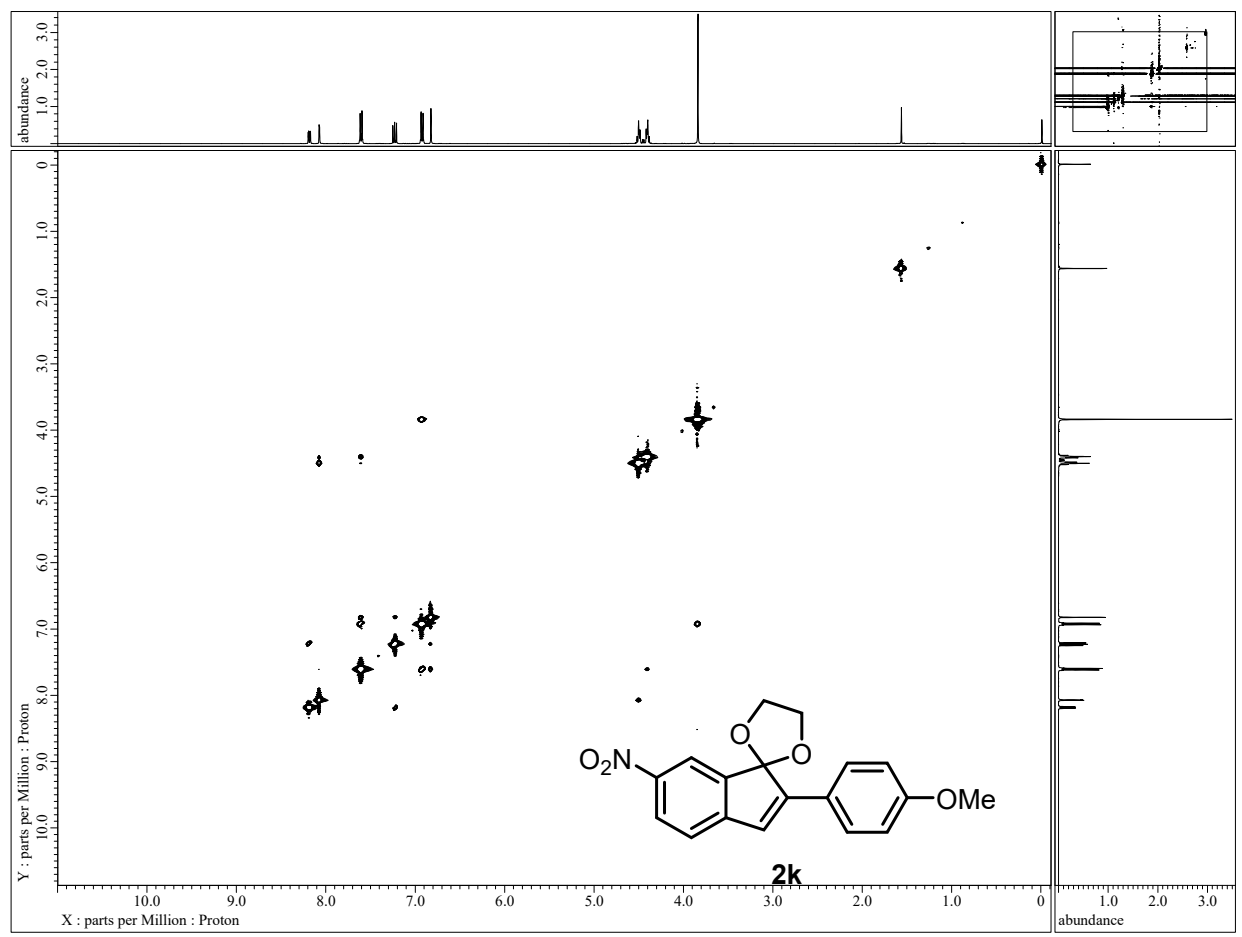


9. ${ }^{1} \mathrm{H},{ }^{2} \mathrm{H}$ and ${ }^{13} \mathrm{C}$ NMR spectra of stating materials and products

${ }^{1} \mathrm{H}$ NMR (400 MHz, $\mathrm{CDCl}_{3}$ ) of $\mathbf{S 1 a}$

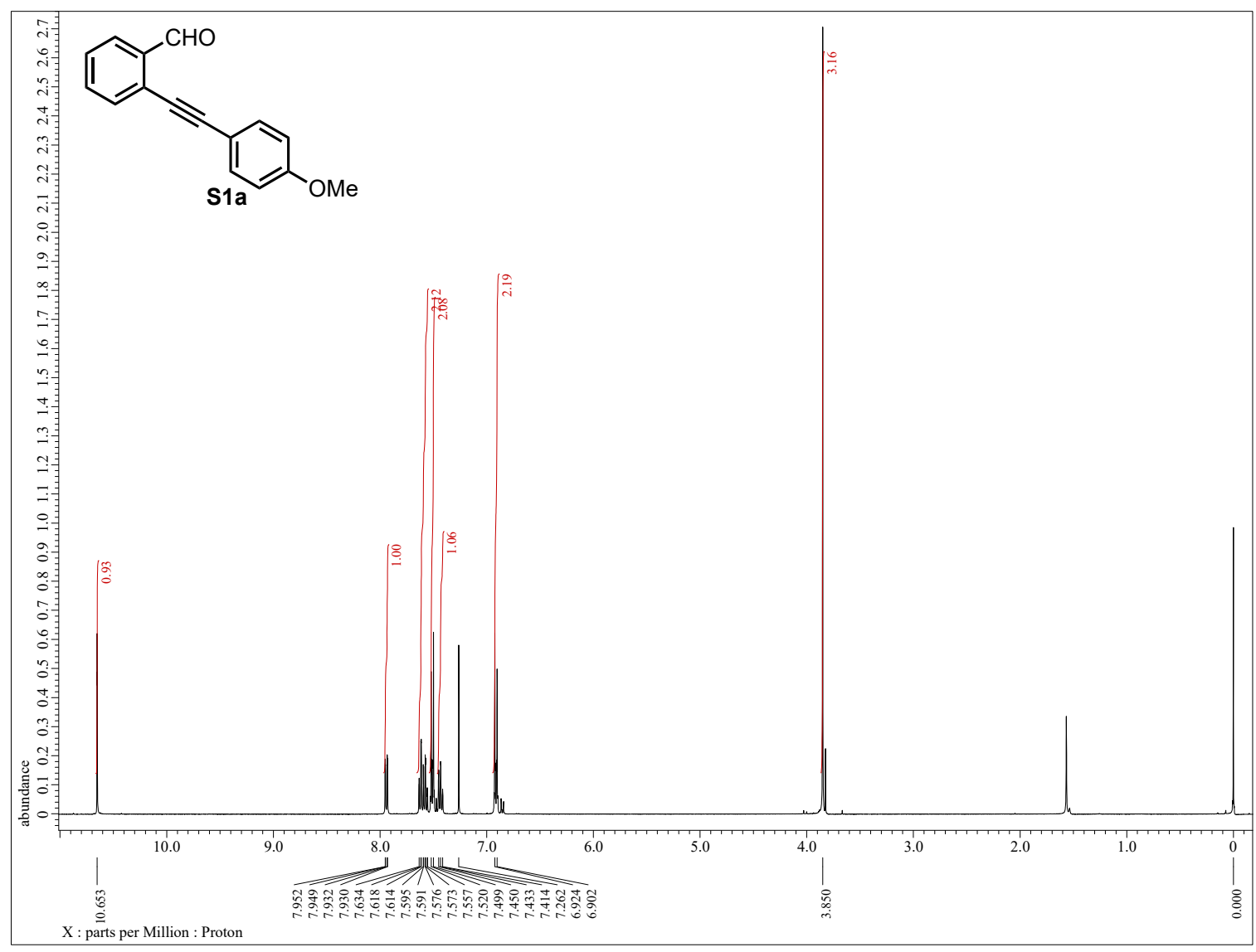

${ }^{1} \mathrm{H}$ NMR (500 MHz, $\mathrm{CDCl}_{3}$ ) of 1a

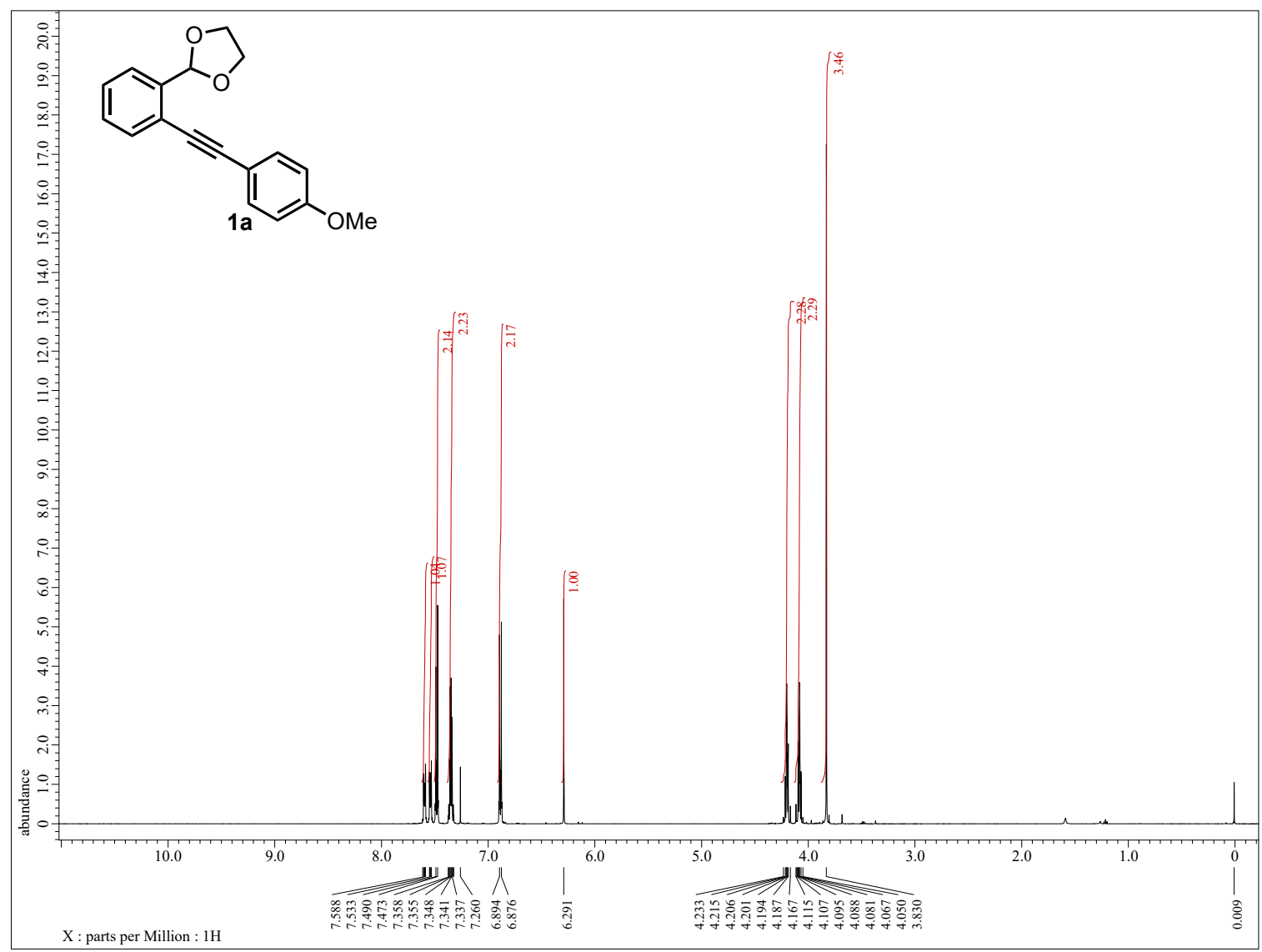


${ }^{13} \mathrm{C}$ NMR $\left(125 \mathrm{MHz}, \mathrm{CDCl}_{3}\right)$ of $\mathbf{1 a}$

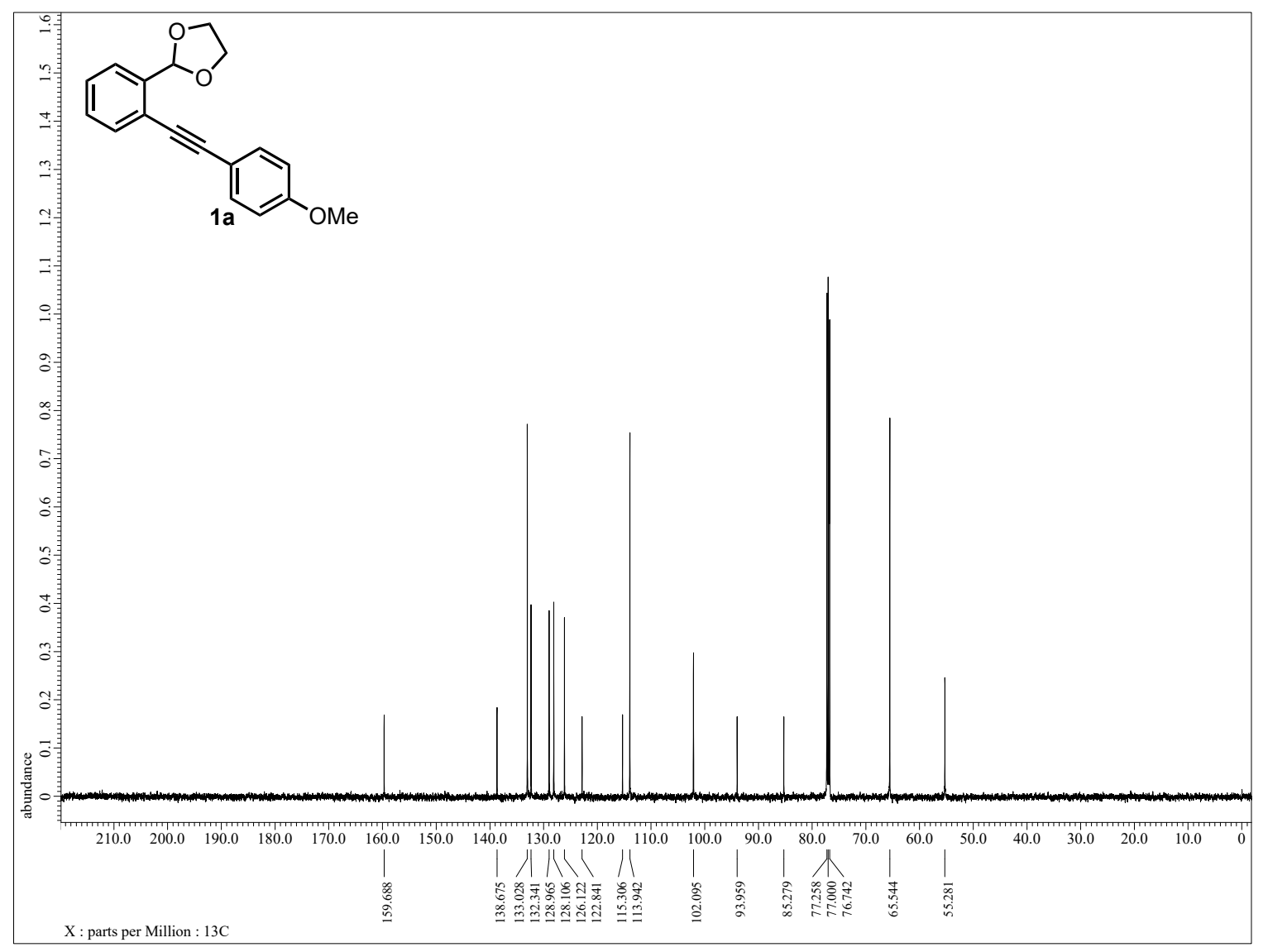

${ }^{1} \mathrm{H}$ NMR (400 MHz, $\mathrm{CDCl}_{3}$ ) of $\mathbf{S 1 b}$

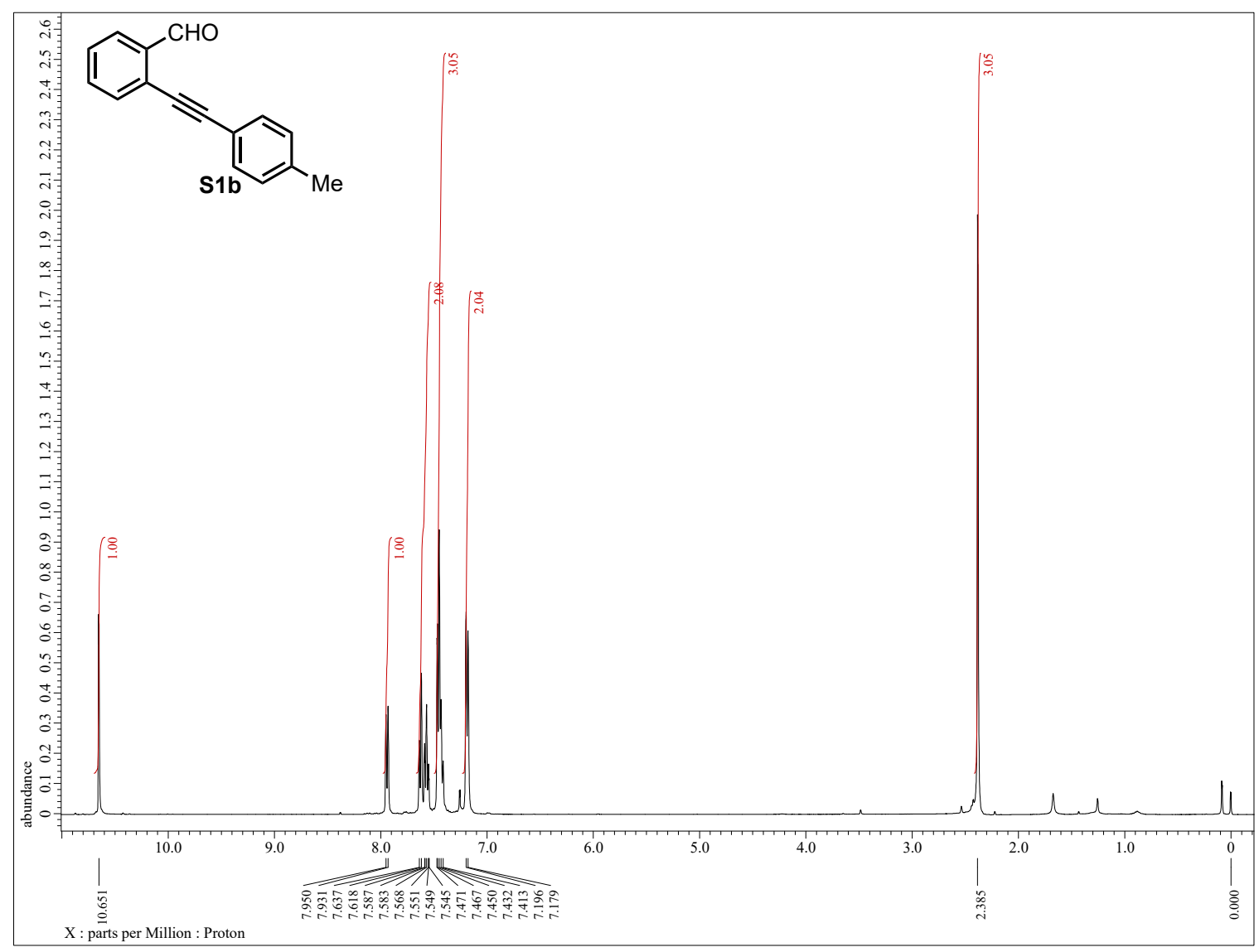

S36 
${ }^{1} \mathrm{H}$ NMR (400 MHz, $\mathrm{CDCl}_{3}$ )of $\mathbf{1 b}$

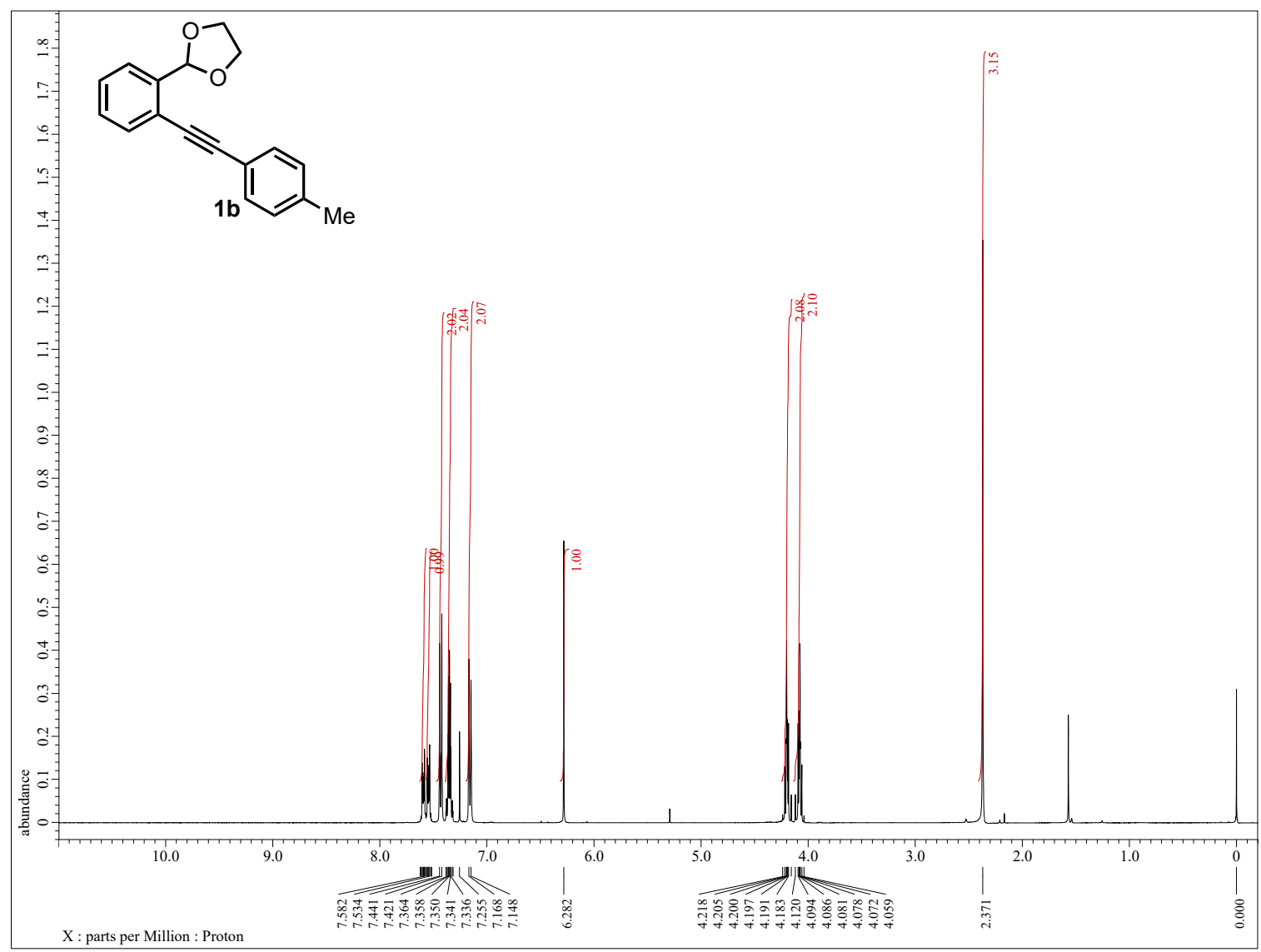

${ }^{13} \mathrm{C}$ NMR $\left(100 \mathrm{MHz}, \mathrm{CDCl}_{3}\right)$ of $\mathbf{1 b}$

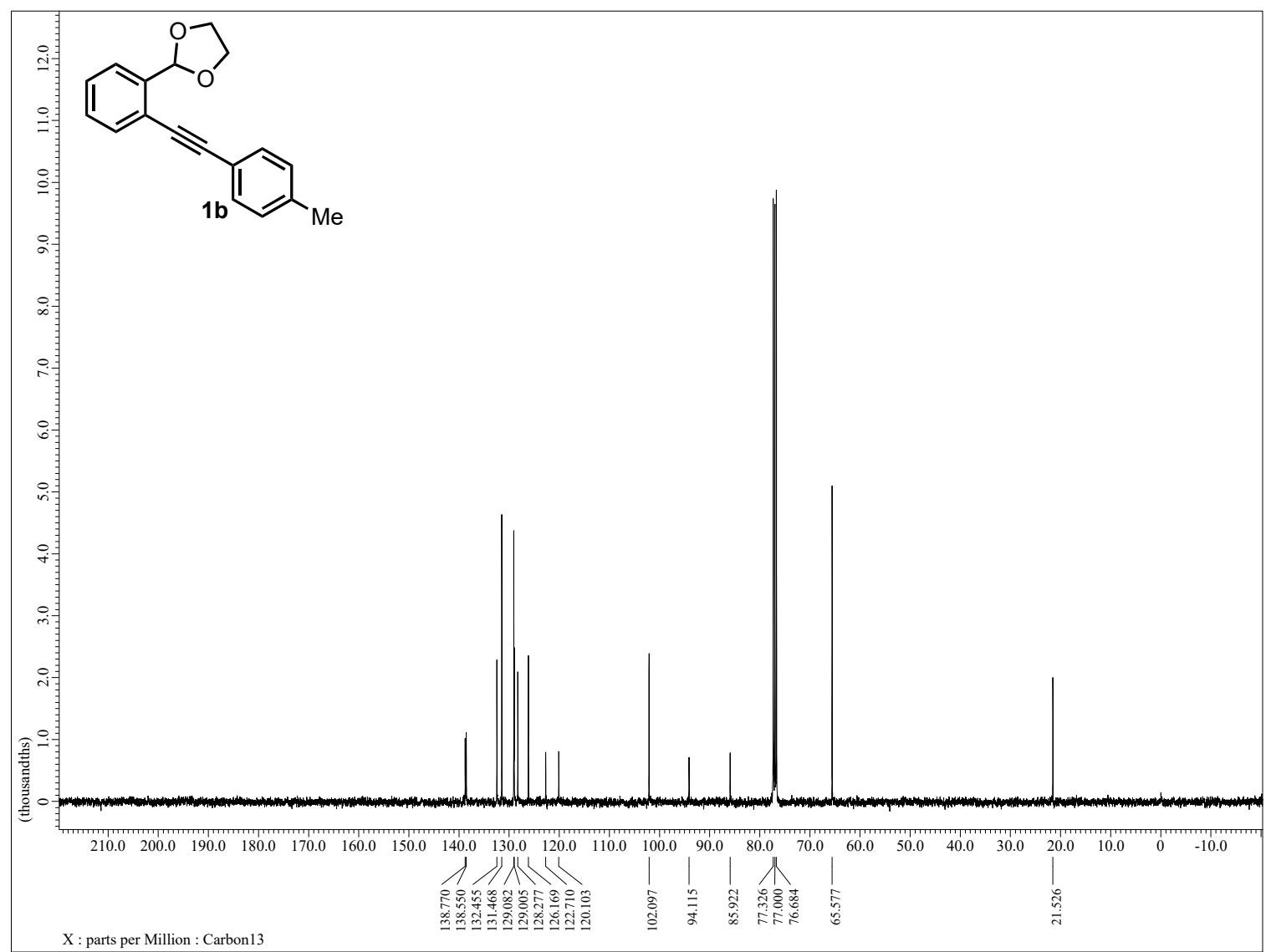


${ }^{1} \mathrm{H}$ NMR (400 MHz, $\mathrm{CDCl}_{3}$ ) of S1c

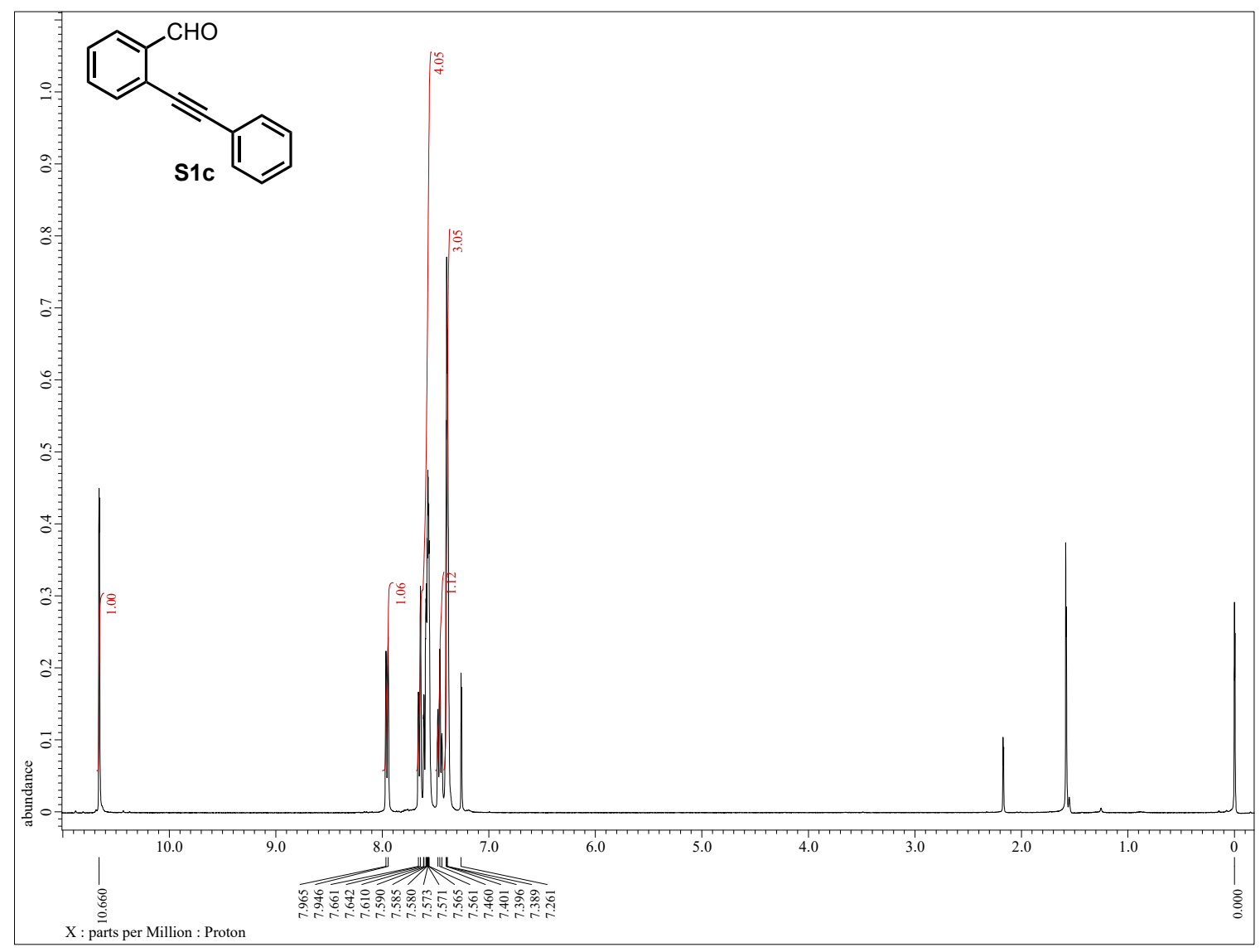

${ }^{1} \mathrm{H}$ NMR (400 MHz, $\mathrm{CDCl}_{3}$ ) of 1c

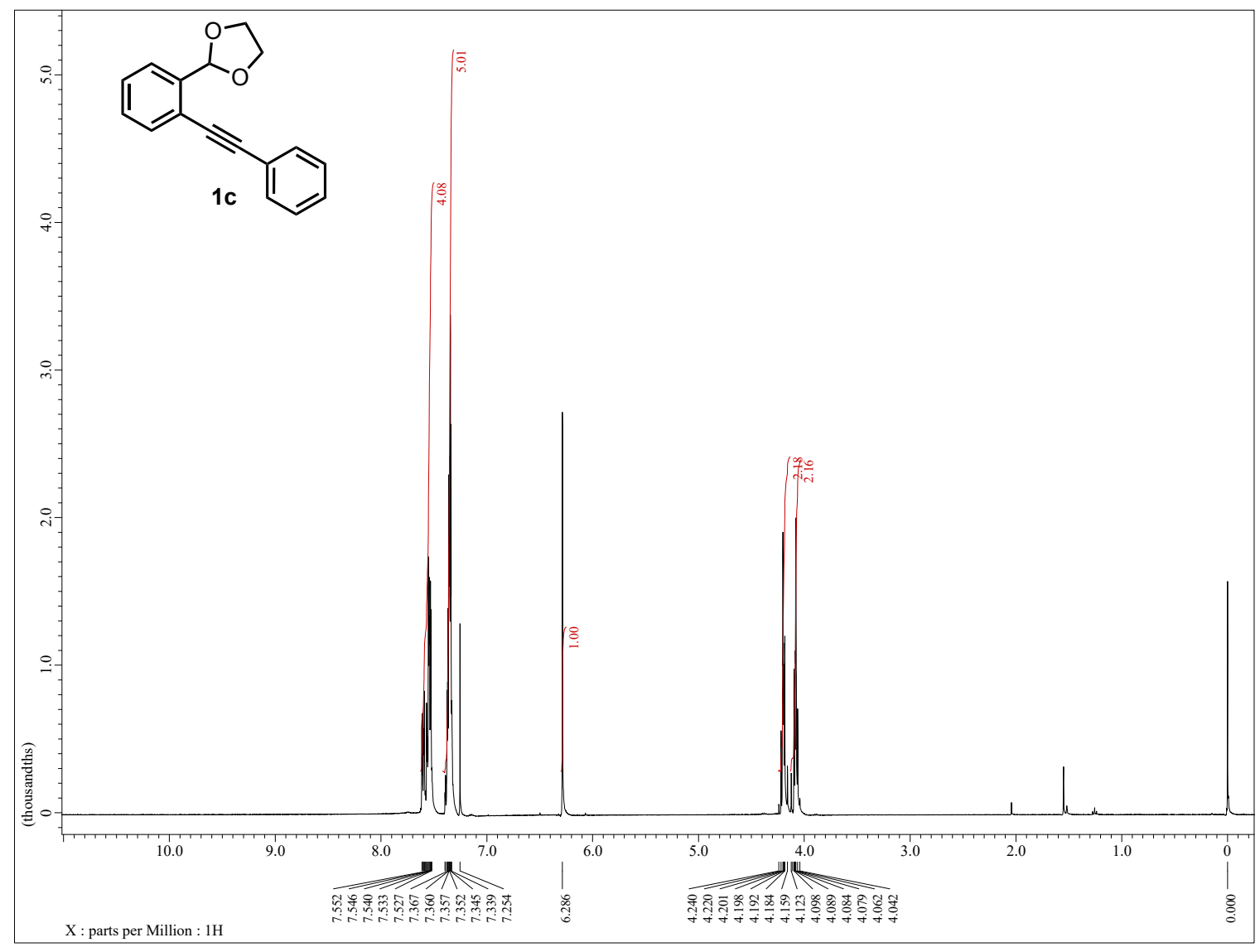

S38 
${ }^{1} \mathrm{H}$ NMR (400 MHz, $\mathrm{CDCl}_{3}$ ) of S1d

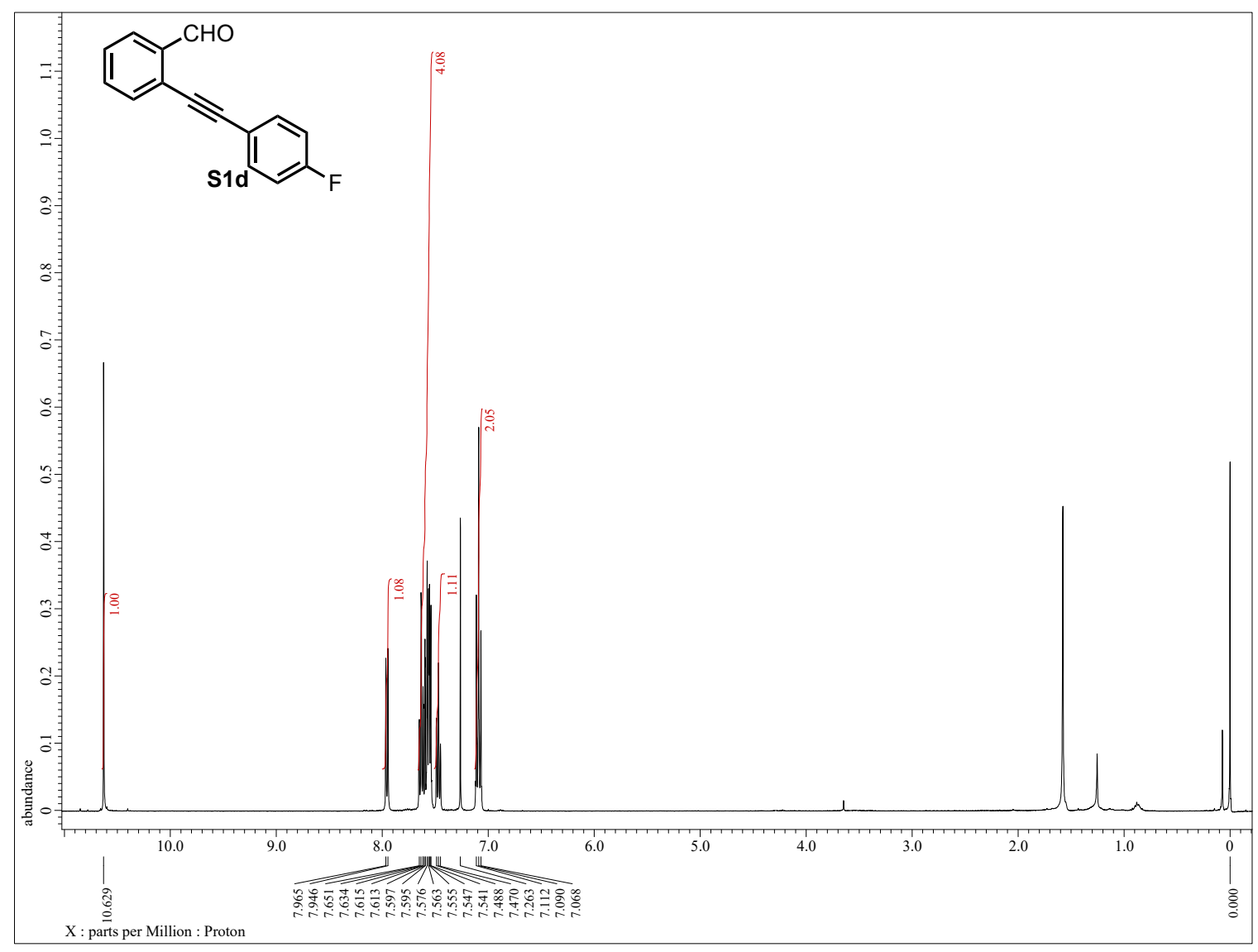

${ }^{1} \mathrm{H}$ NMR (400 MHz, $\mathrm{CDCl}_{3}$ ) of $\mathbf{1 d}$

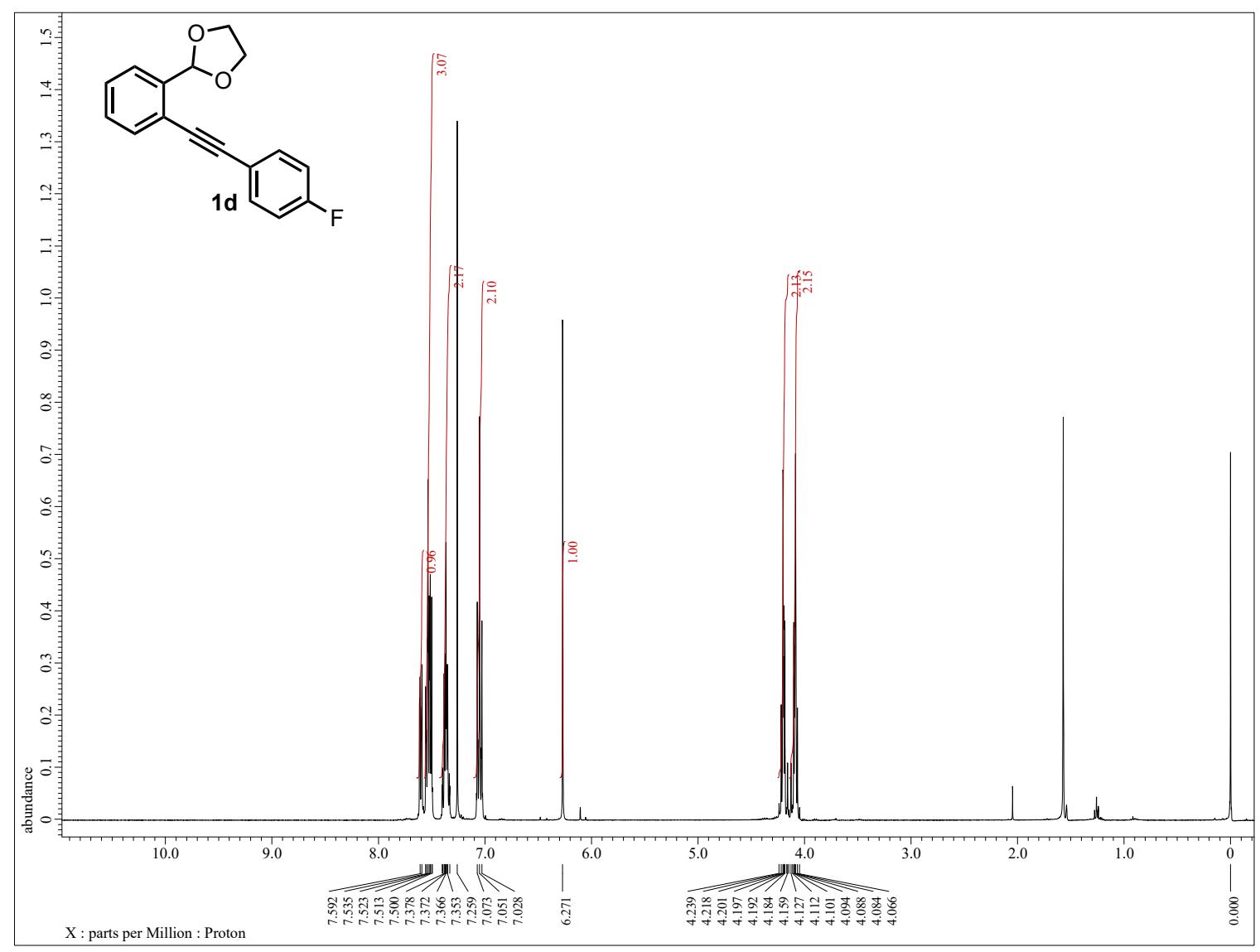

S39 
${ }^{13} \mathrm{C}$ NMR $\left(100 \mathrm{MHz}, \mathrm{CDCl}_{3}\right)$ of $\mathbf{1 d}$

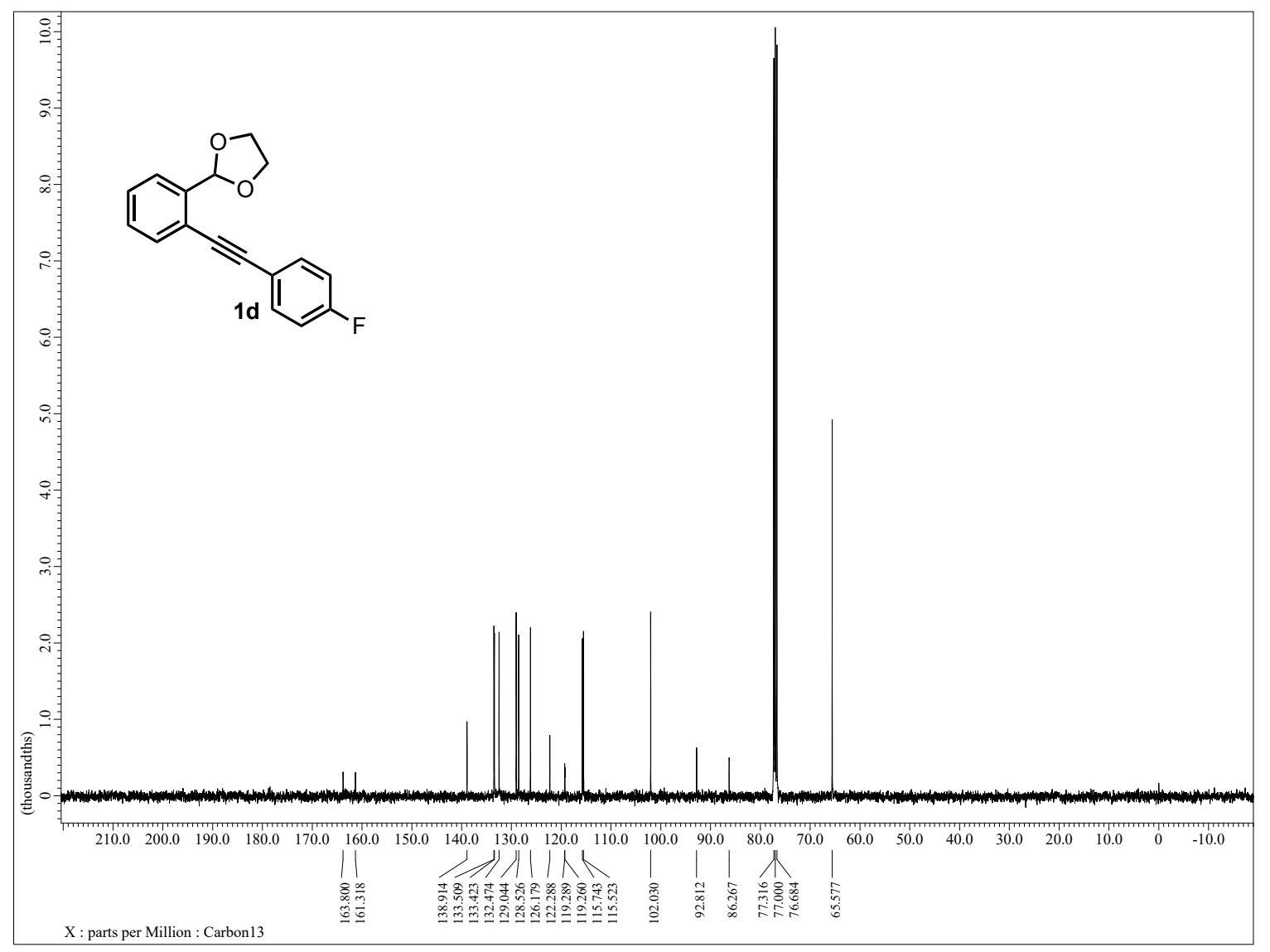

${ }^{1} \mathrm{H}$ NMR (400 MHz, $\mathrm{CDCl}_{3}$ ) of STe

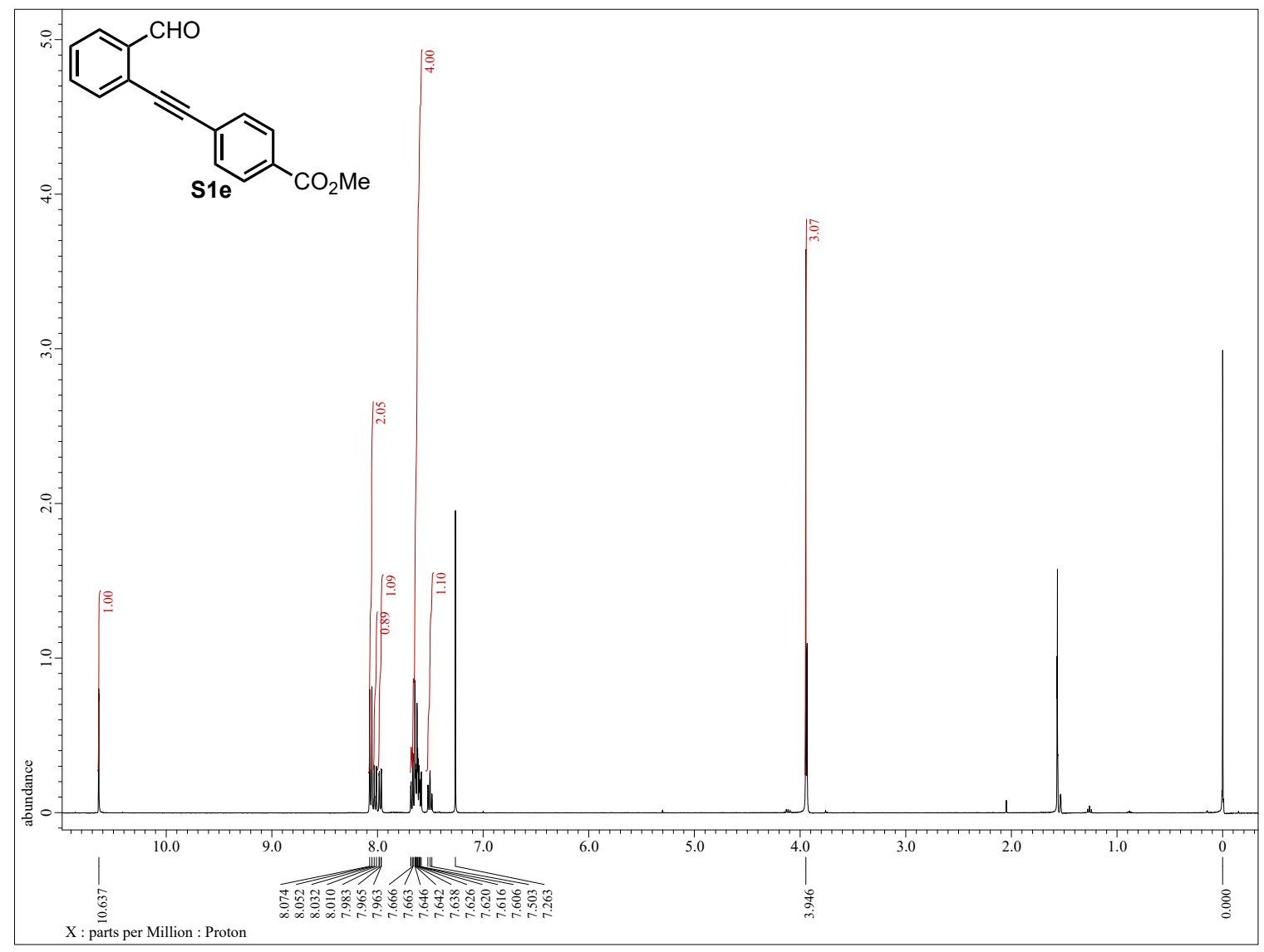

$\mathrm{S} 40$ 
${ }^{1} \mathrm{H}$ NMR (400 MHz, $\mathrm{CDCl}_{3}$ ) of ie

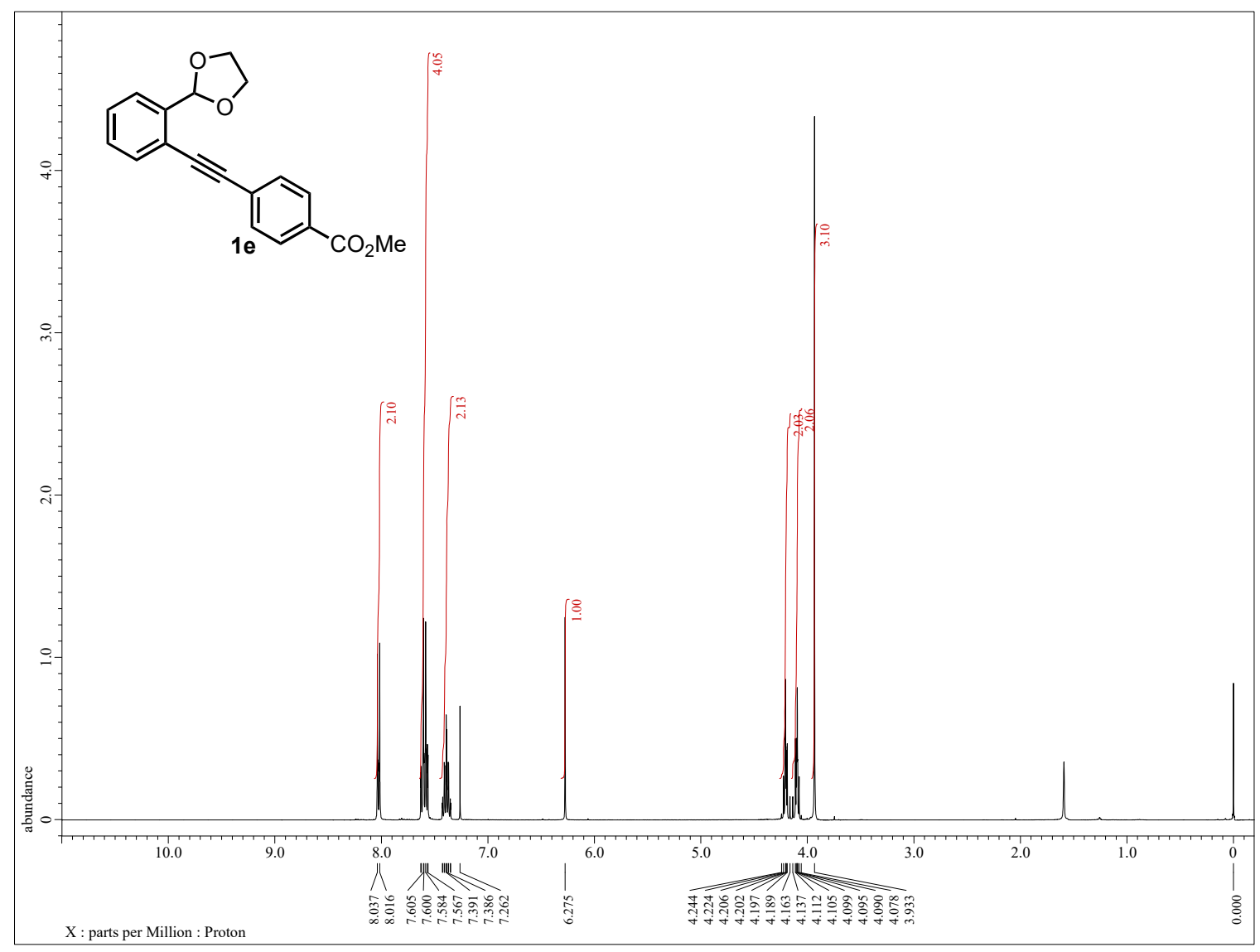

${ }^{13} \mathrm{C}$ NMR $\left(100 \mathrm{MHz}, \mathrm{CDCl}_{3}\right)$ of $\mathbf{1 e}$

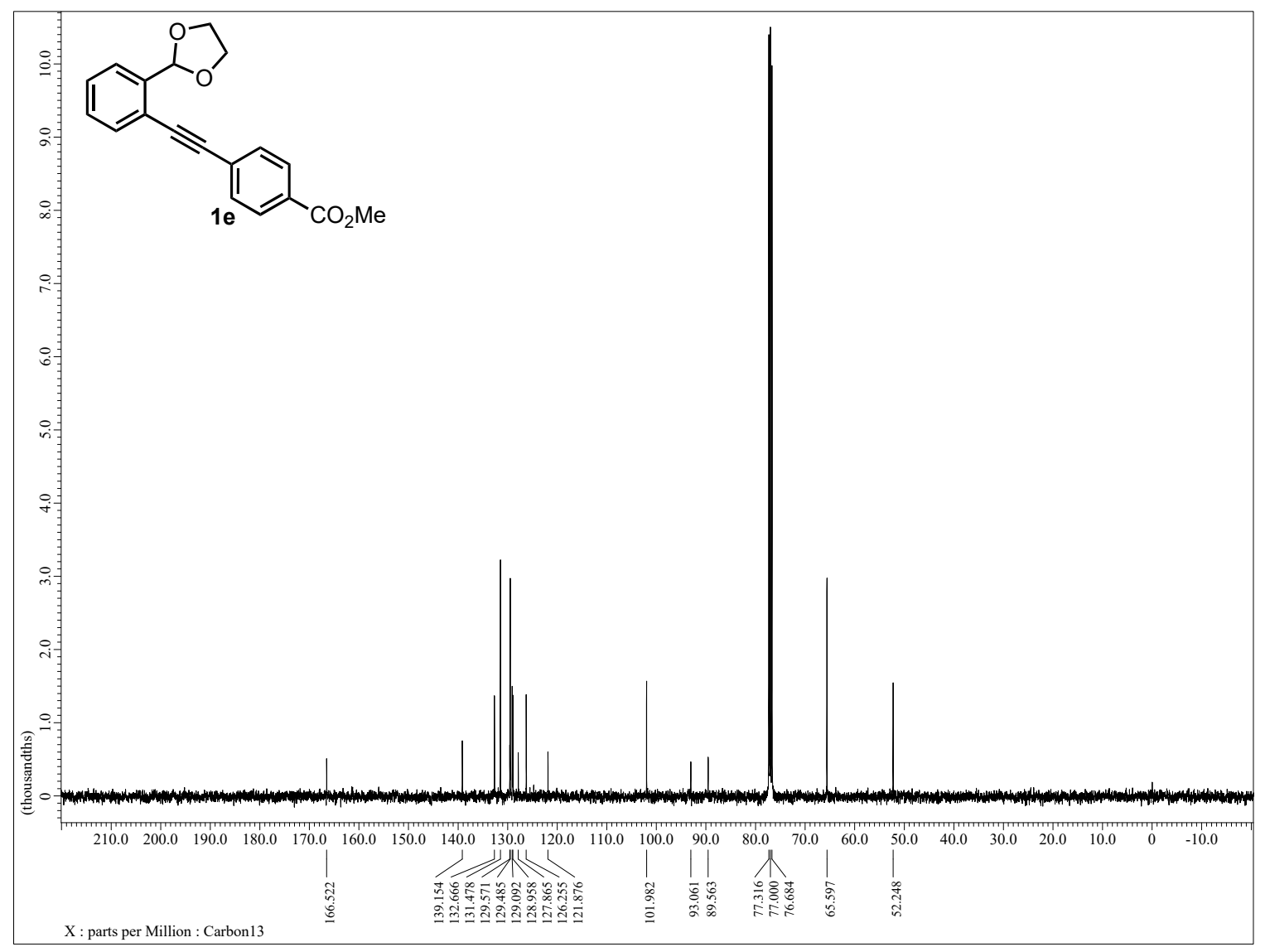

S41 
${ }^{1} \mathrm{H}$ NMR (400 MHz, $\mathrm{CDCl}_{3}$ ) of S1f

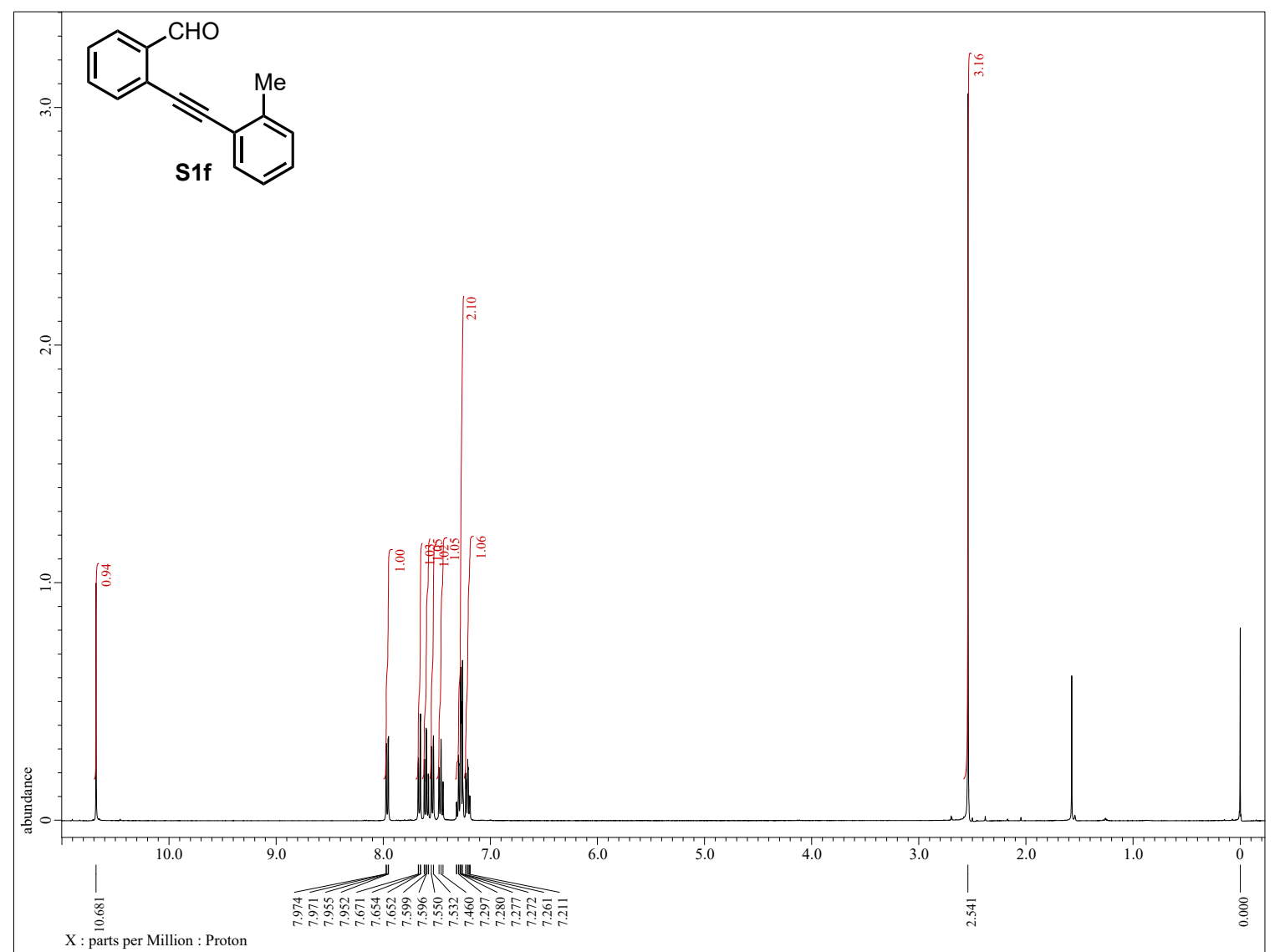

${ }^{1} \mathrm{H}$ NMR (400 MHz, $\mathrm{CDCl}_{3}$ ) of $\mathbf{1 f}$

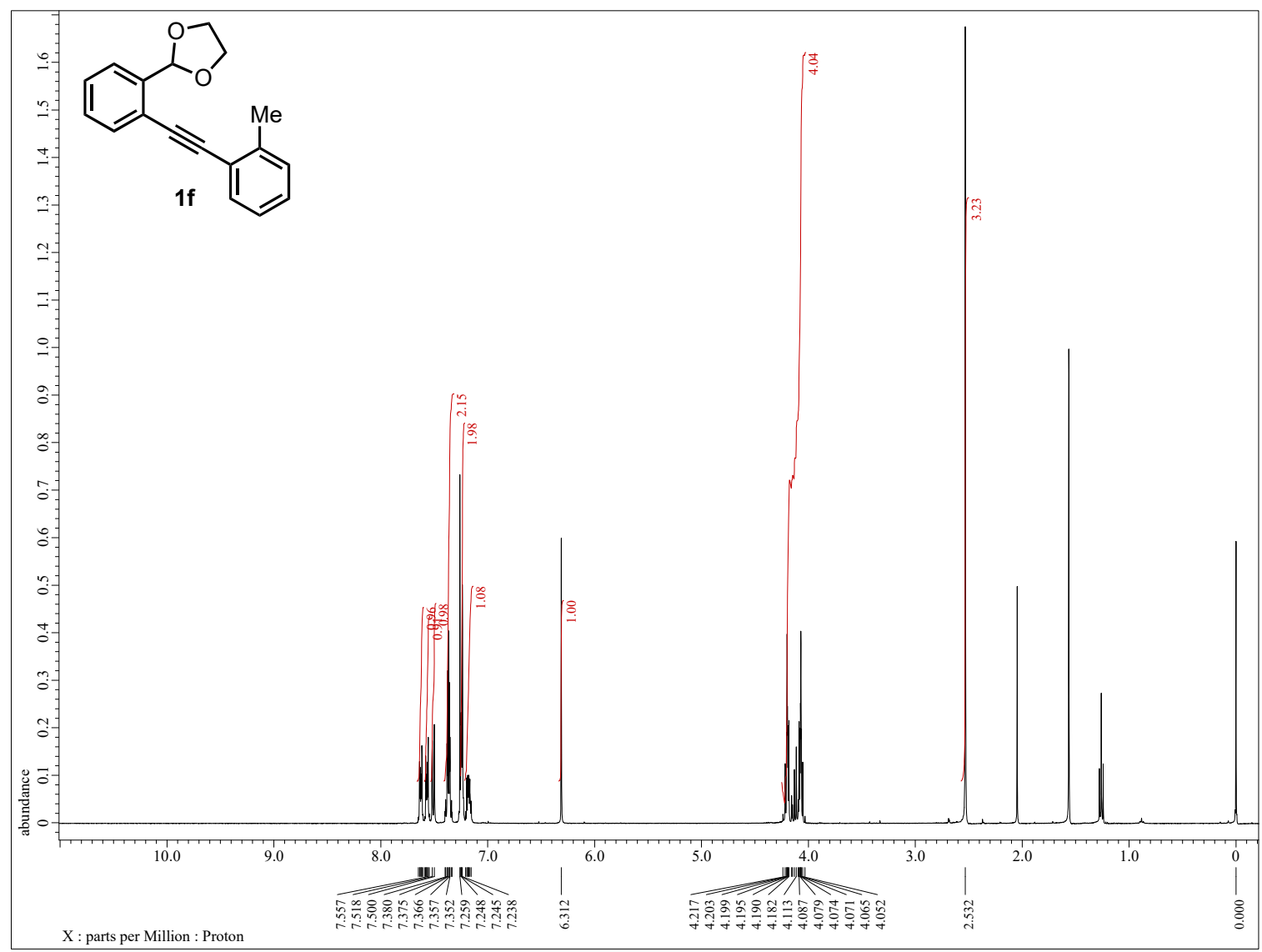


${ }^{13} \mathrm{C}$ NMR $\left(100 \mathrm{MHz}, \mathrm{CDCl}_{3}\right)$ of $\mathbf{1 f}$

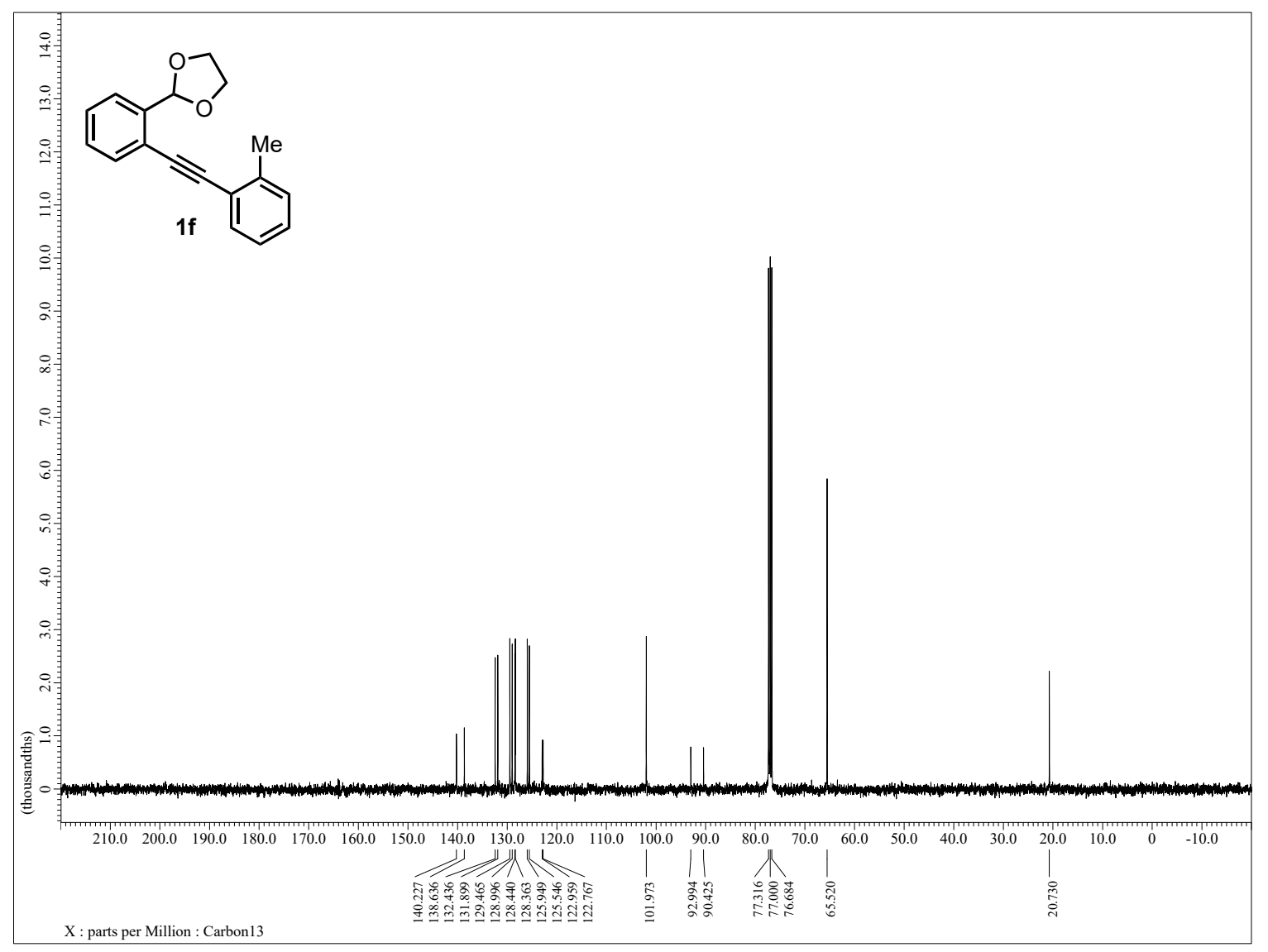

${ }^{1} \mathrm{H}$ NMR (400 MHz, $\mathrm{CDCl}_{3}$ ) of $\mathbf{S 1 g}$

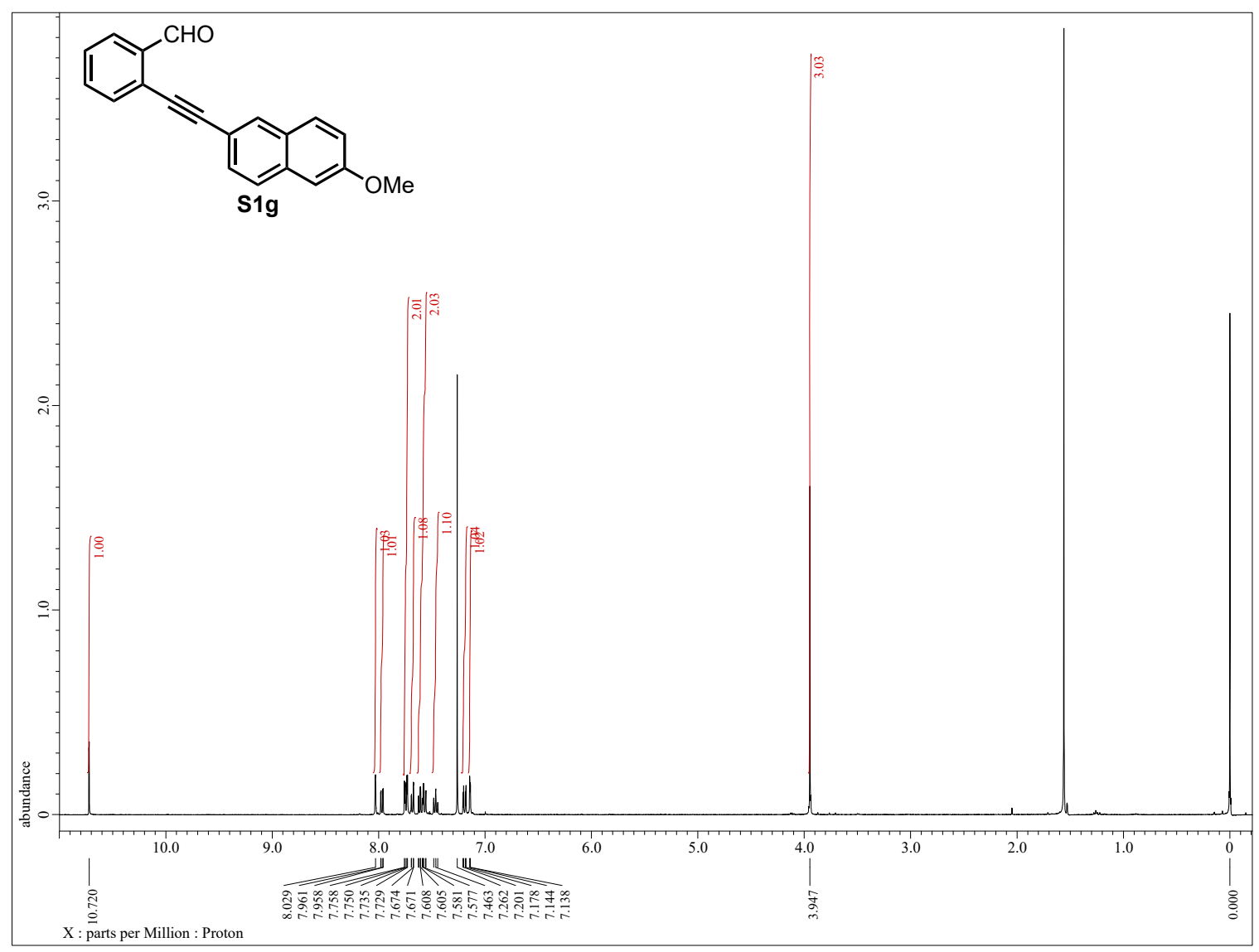

S43 
${ }^{1} \mathrm{H}$ NMR (400 MHz, $\mathrm{CDCl}_{3}$ ) of $\mathbf{1 g}$

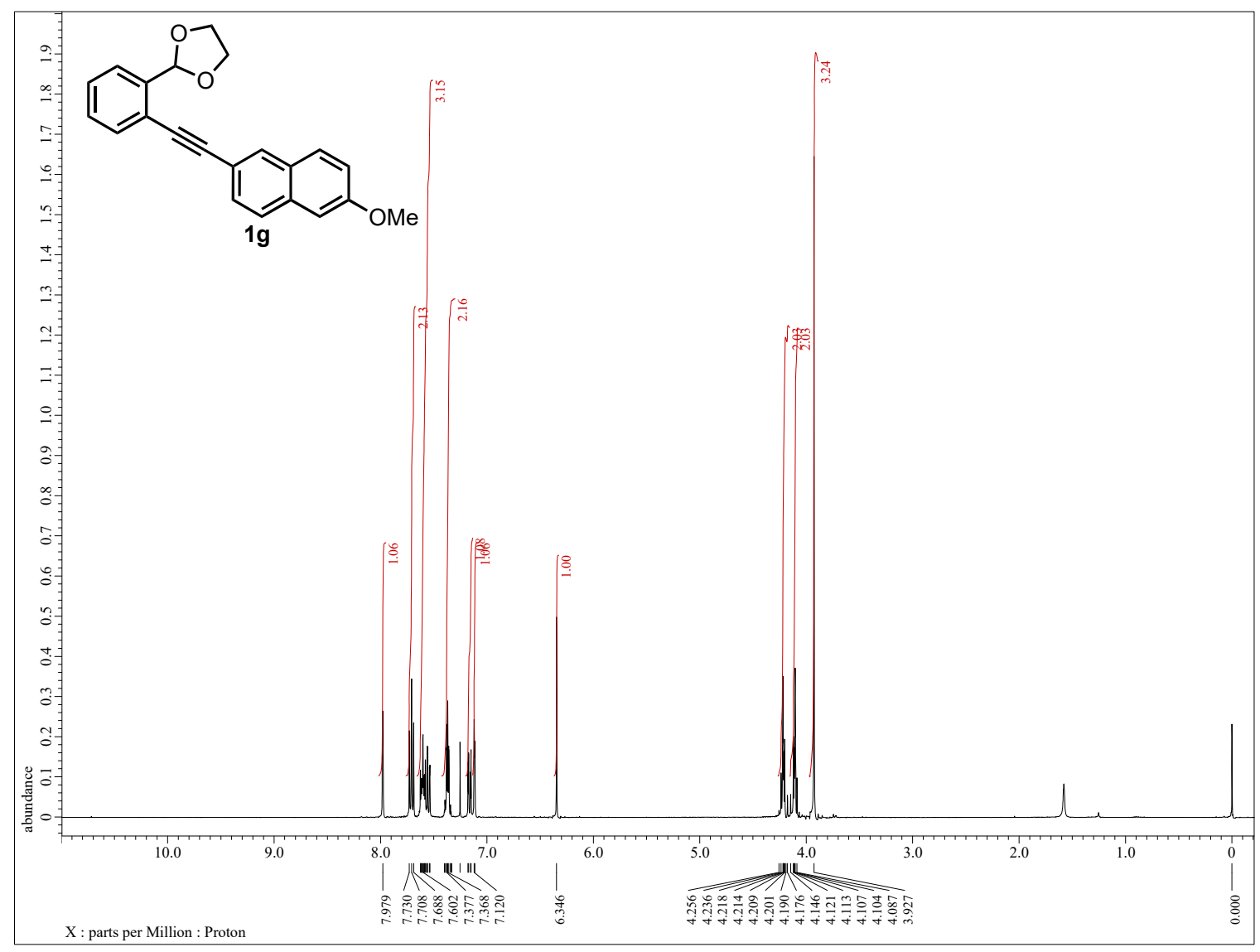

${ }^{13} \mathrm{C}$ NMR (100 MHz, $\mathrm{CDCl}_{3}$ ) of $\mathbf{1 g}$

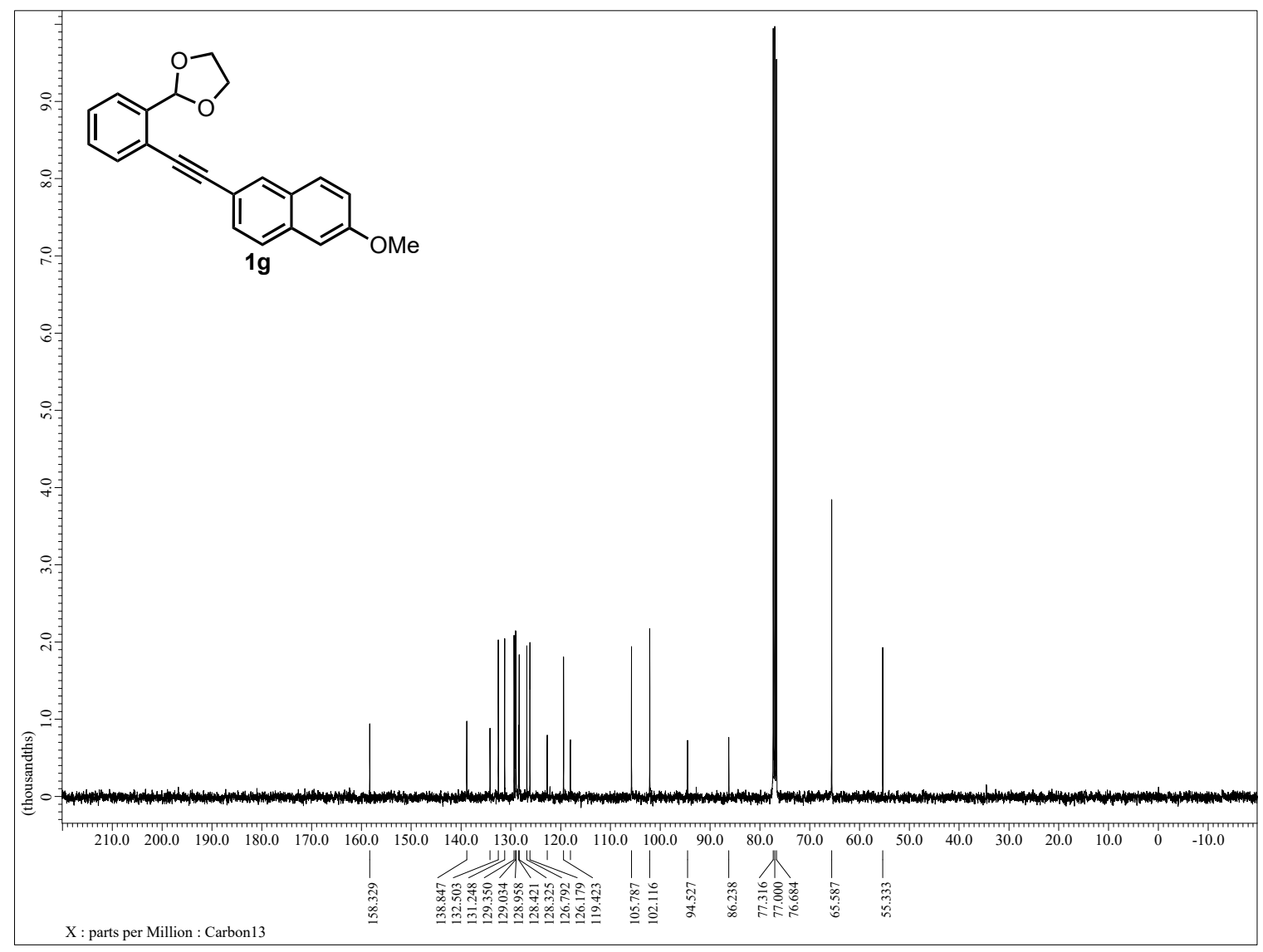

S44 
${ }^{1} \mathrm{H}$ NMR (400 MHz, $\mathrm{CDCl}_{3}$ ) of $\mathbf{S 1 h}$

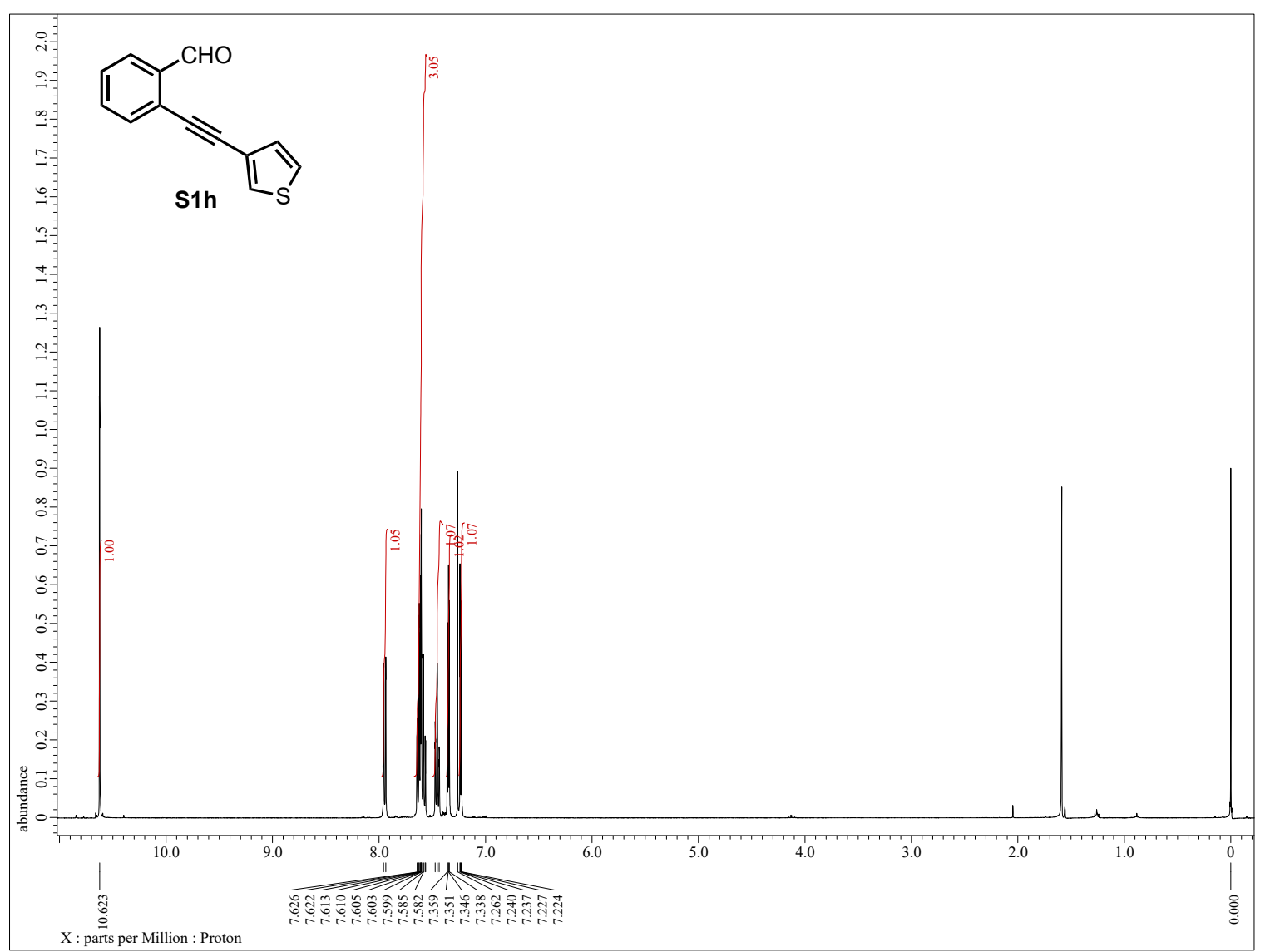

${ }^{1} \mathrm{H}$ NMR (400 MHz, $\mathrm{CDCl}_{3}$ ) of $\mathbf{1 h}$

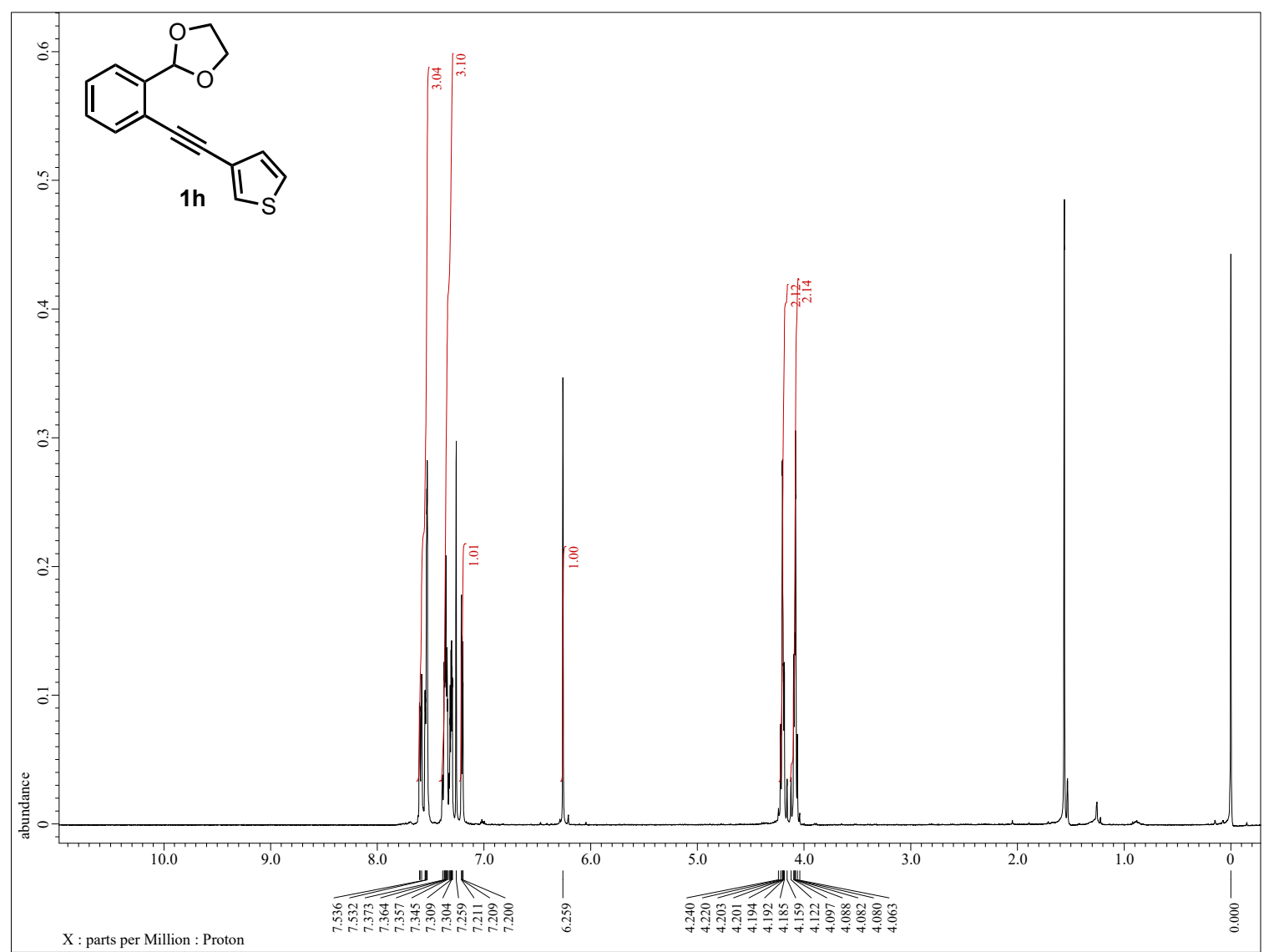


${ }^{13} \mathrm{C}$ NMR (400 MHz, $\mathrm{CDCl}_{3}$ ) of $\mathbf{1 h}$

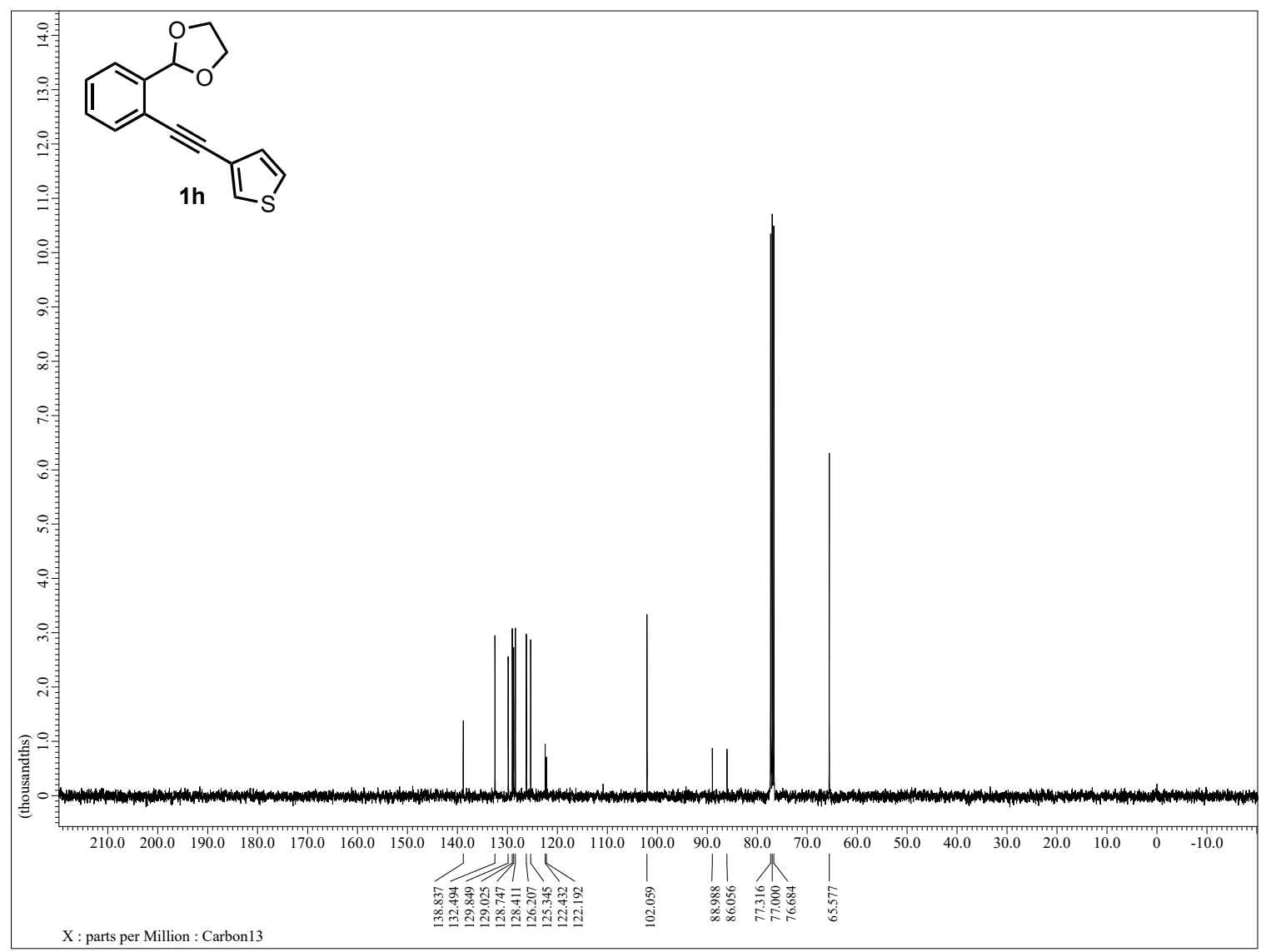

${ }^{1} \mathrm{H}$ NMR (400 MHz, $\mathrm{CDCl}_{3}$ ) of $\mathbf{S 1 i}$

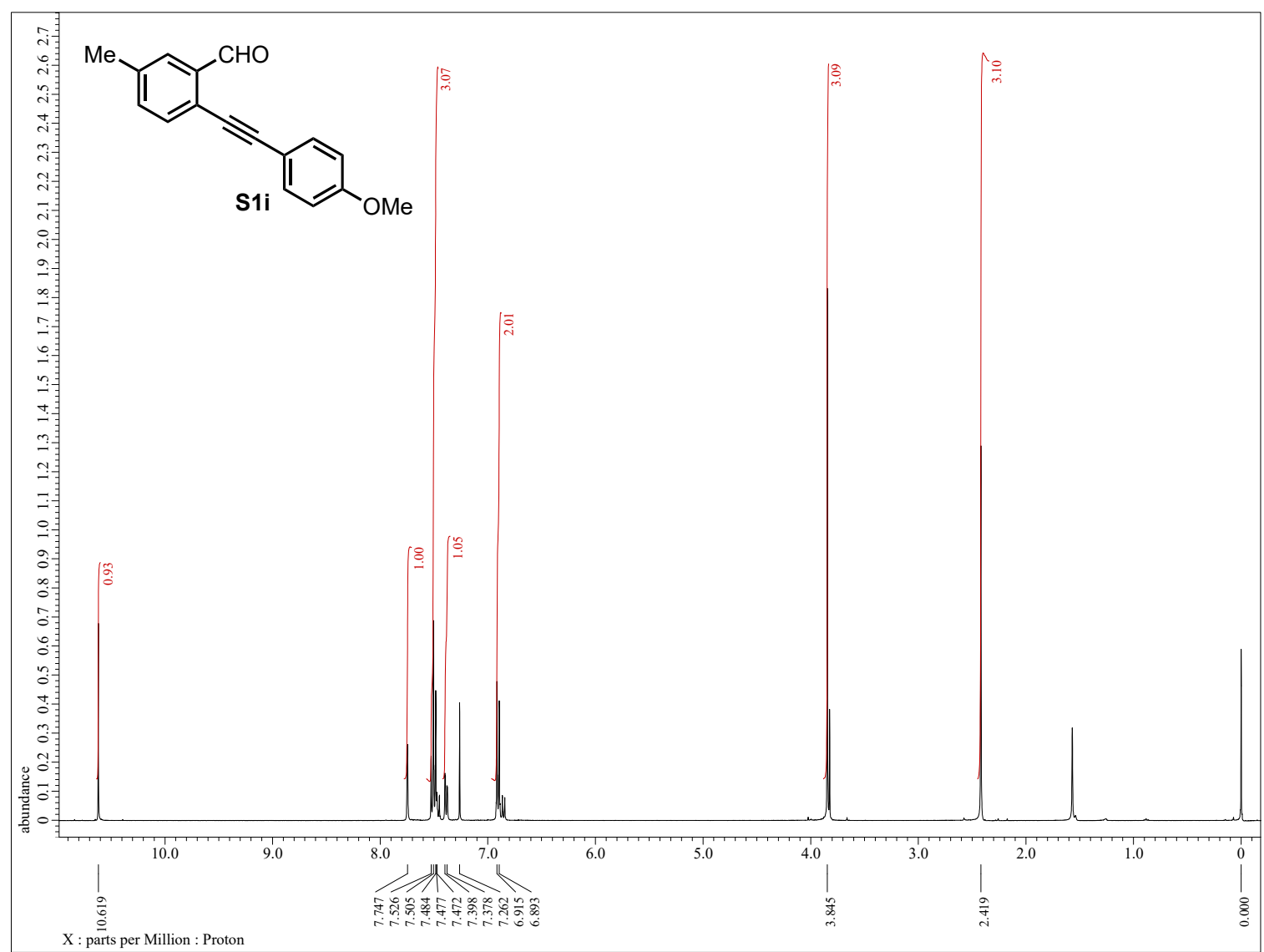


${ }^{1} \mathrm{H}$ NMR $\left(500 \mathrm{MHz}, \mathrm{CDCl}_{3}\right)$ of $\mathbf{1 i}$

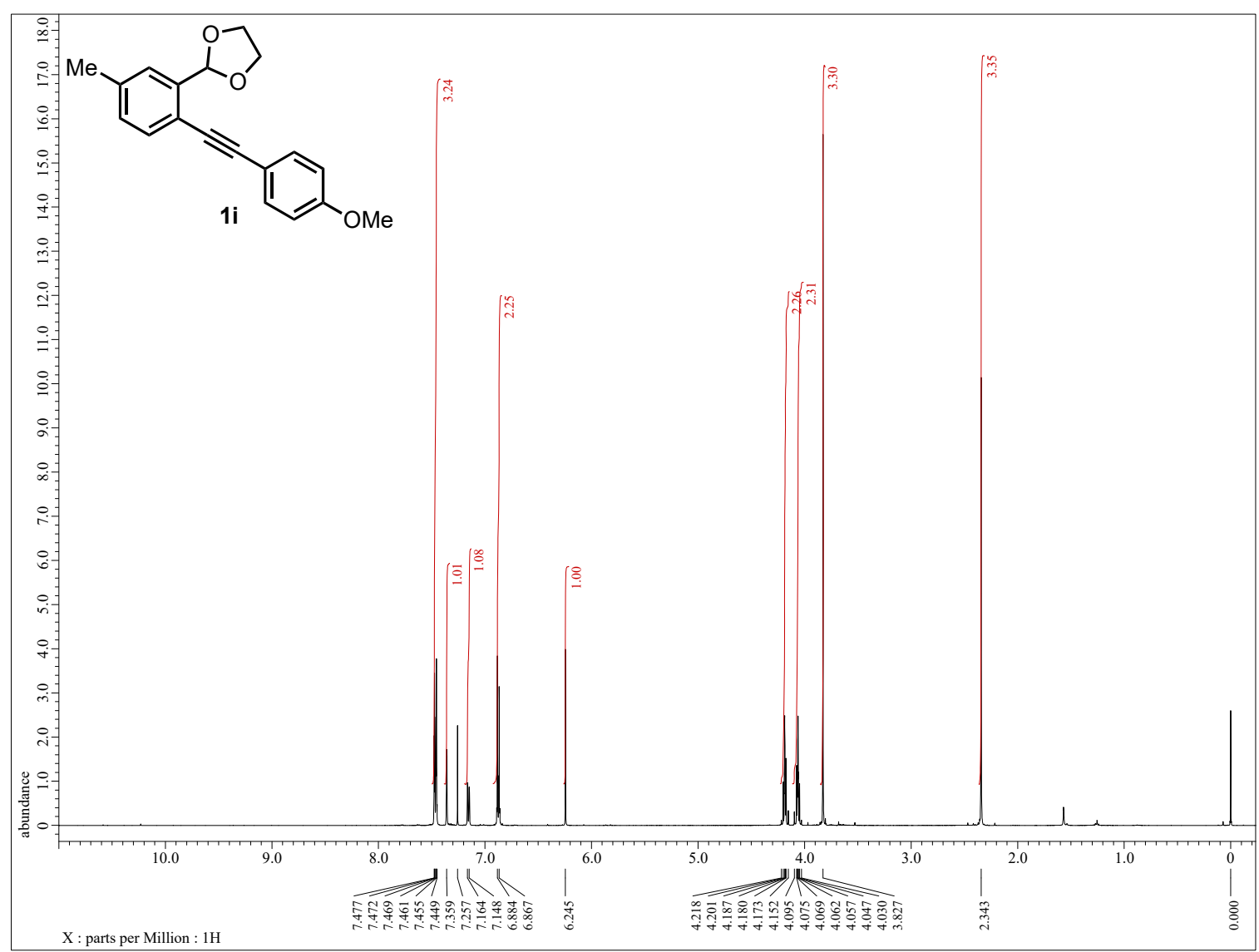

${ }^{13} \mathrm{C} \mathrm{NMR}\left(125 \mathrm{MHz}, \mathrm{CDCl}_{3}\right)$ of $\mathbf{1 i}$

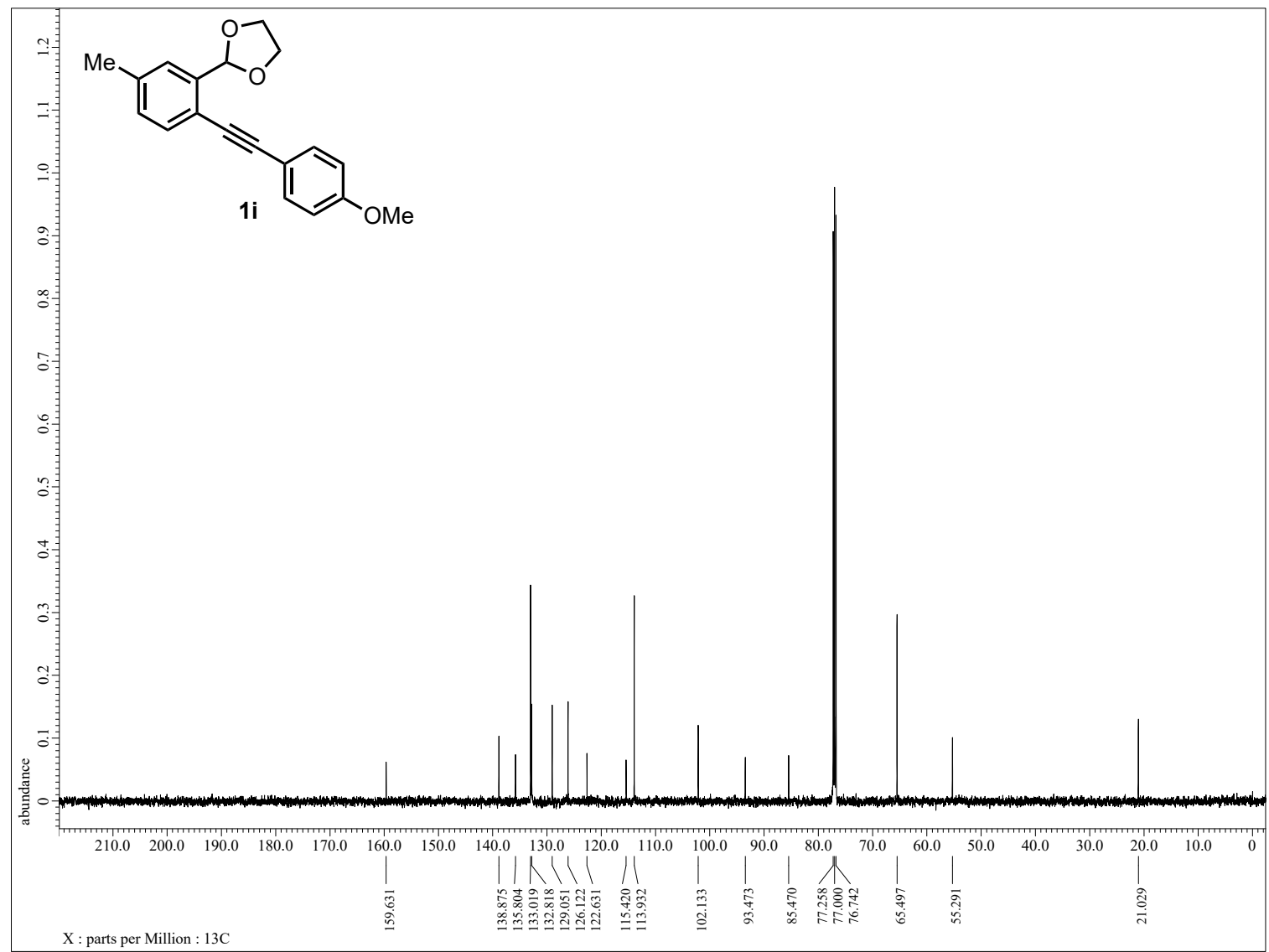


${ }^{1} \mathrm{H}$ NMR $\left(500 \mathrm{MHz}, \mathrm{CDCl}_{3}\right)$ of $\mathbf{S 1 j}$

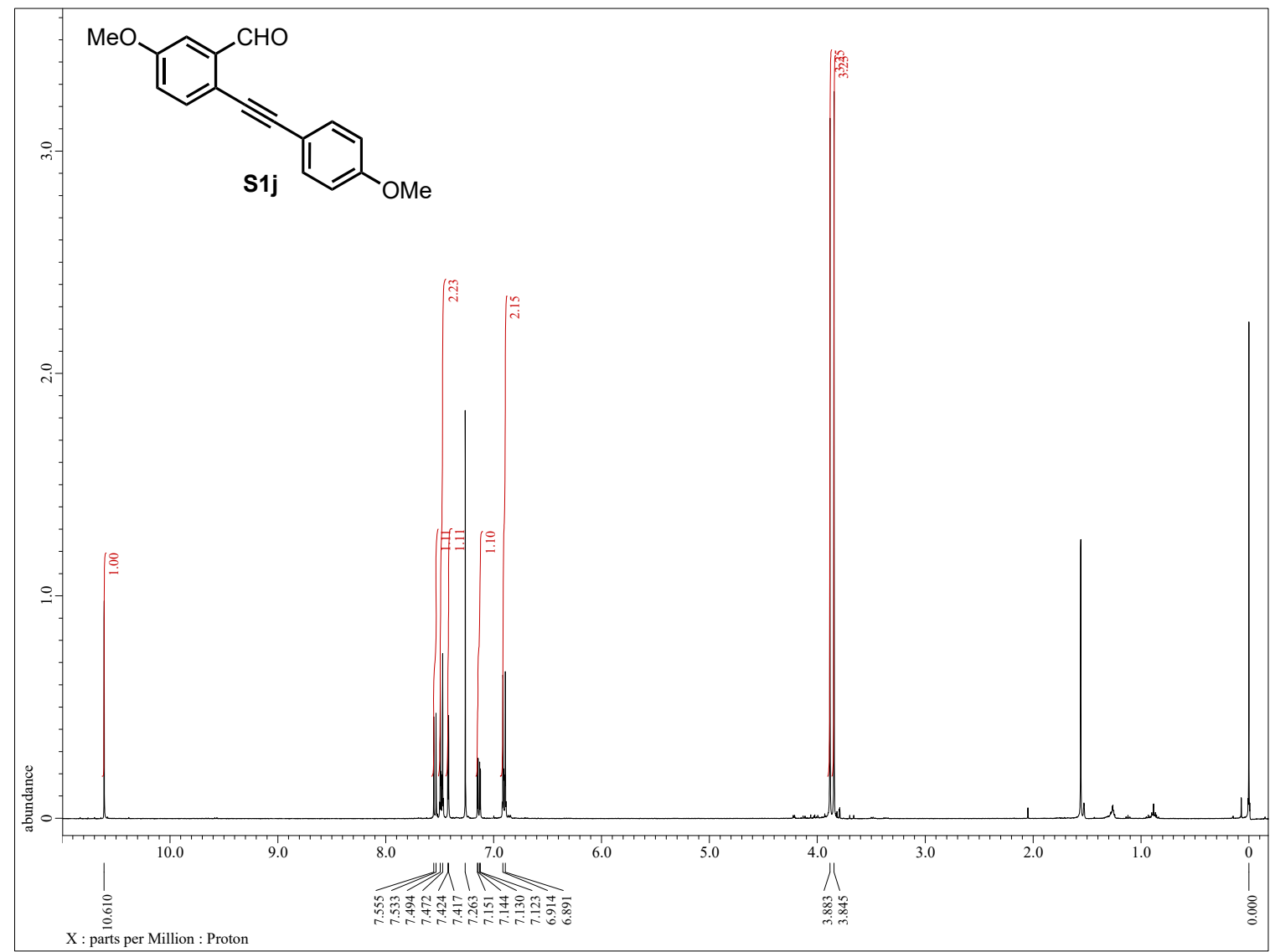

${ }^{1} \mathrm{H}$ NMR $\left(500 \mathrm{MHz}, \mathrm{CDCl}_{3}\right)$ of $\mathbf{1} \mathbf{j}$

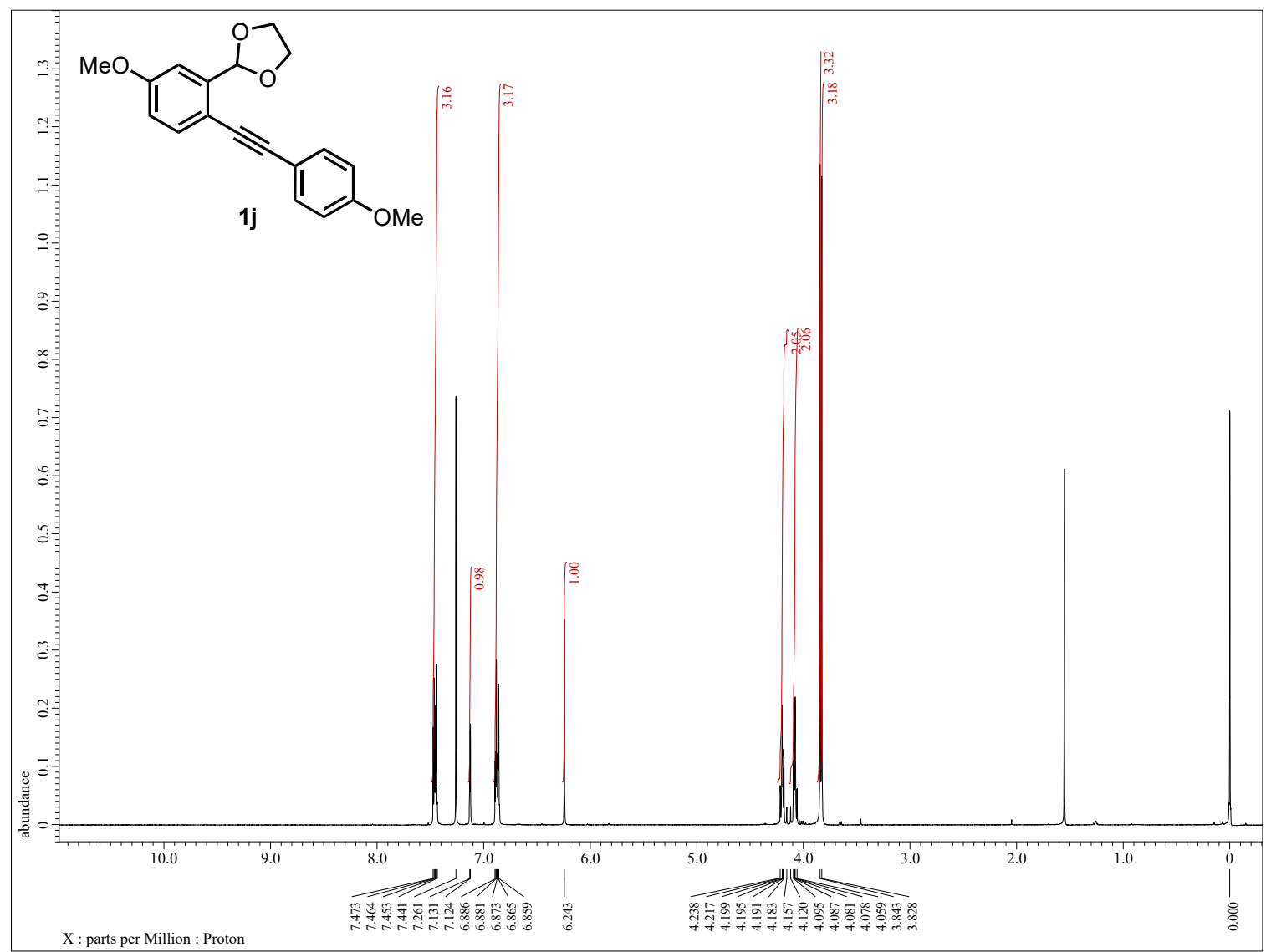


${ }^{13} \mathrm{C}$ NMR $\left(125 \mathrm{MHz}, \mathrm{CDCl}_{3}\right)$ of $\mathbf{1 j}$

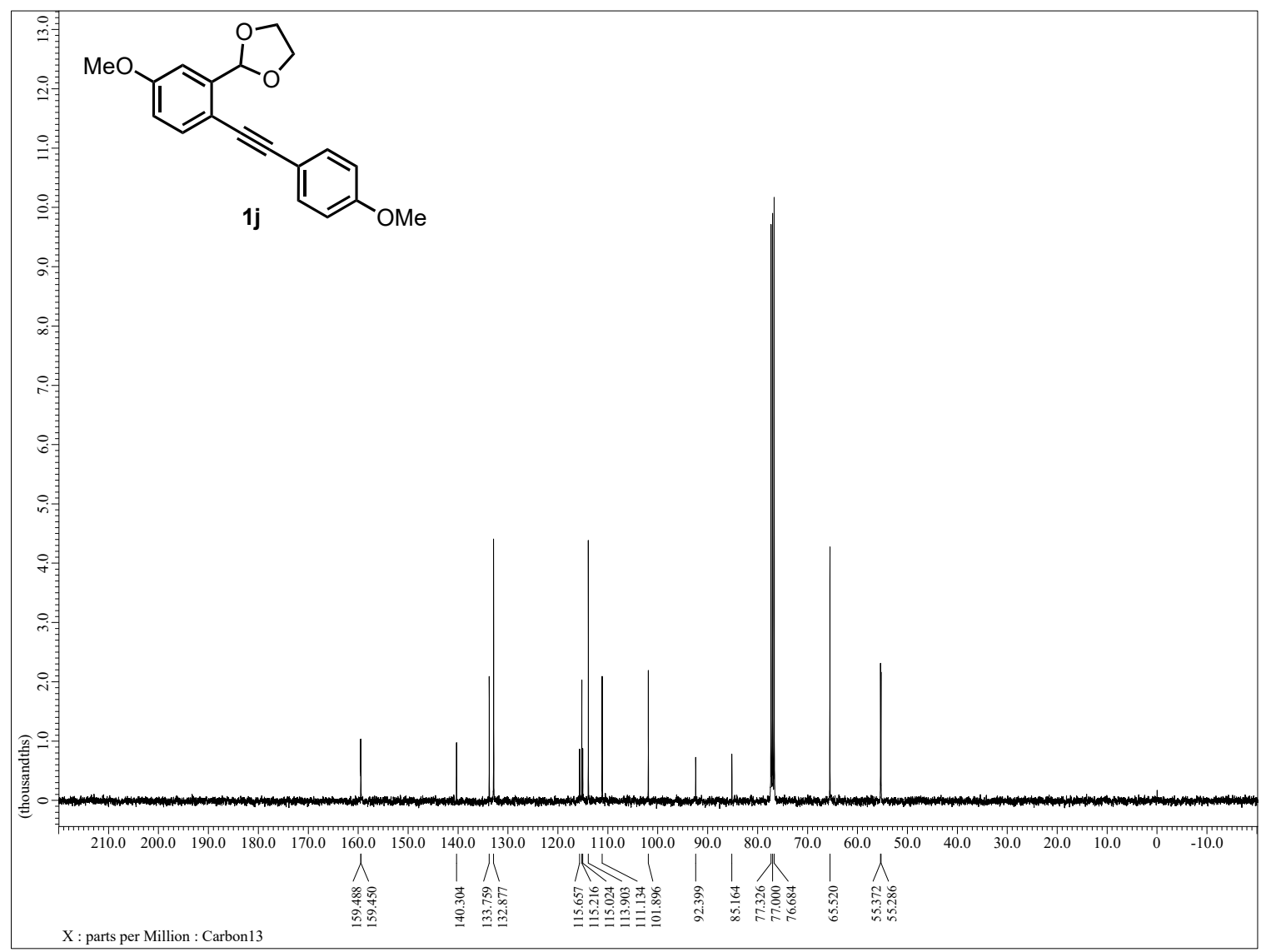

${ }^{1} \mathrm{H}$ NMR (500 MHz, $\mathrm{CDCl}_{3}$ ) of S1k-1

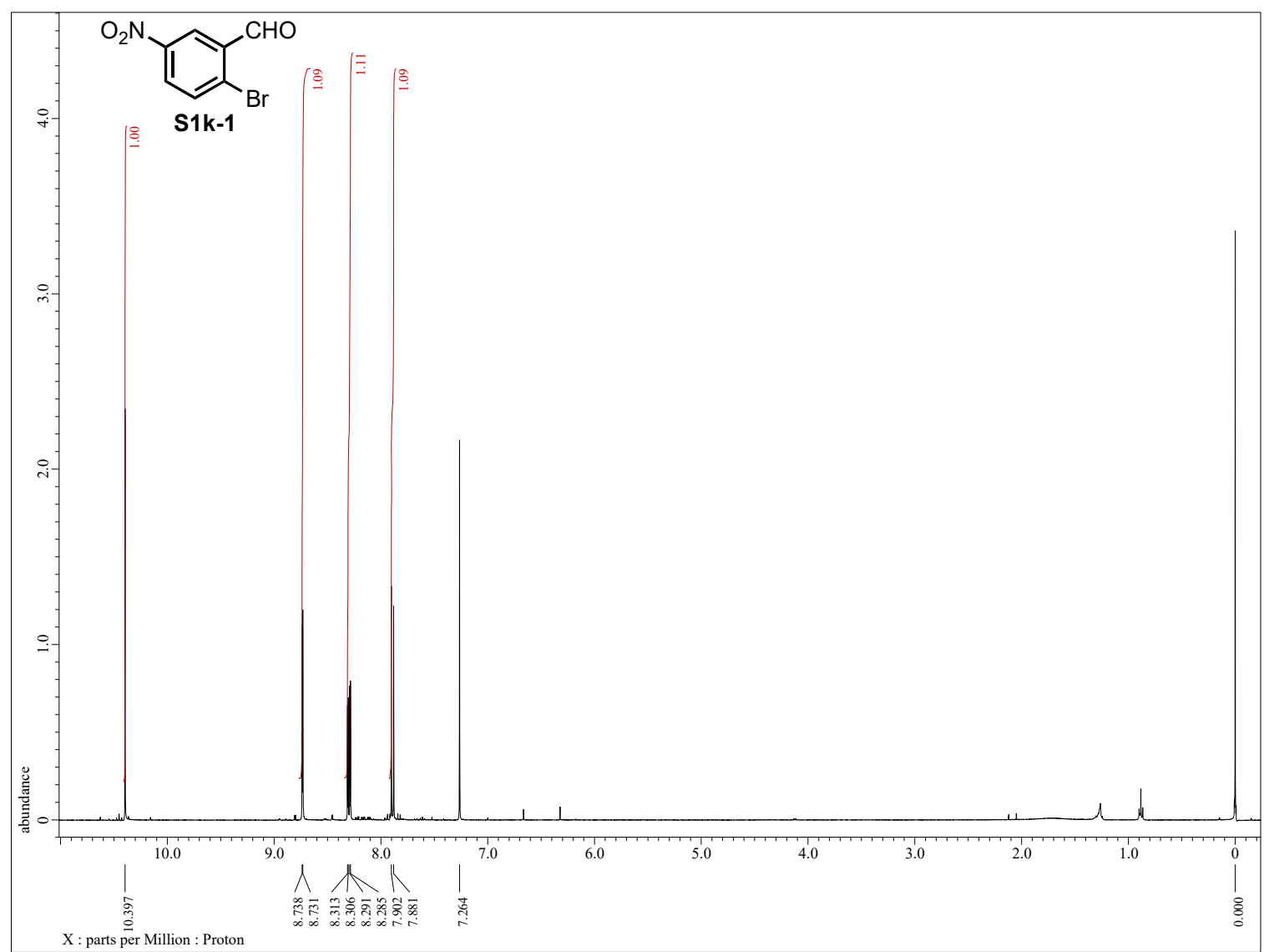


${ }^{1} \mathrm{H}$ NMR (400 MHz, $\mathrm{CDCl}_{3}$ ) of $\mathbf{S 1 k - 2}$

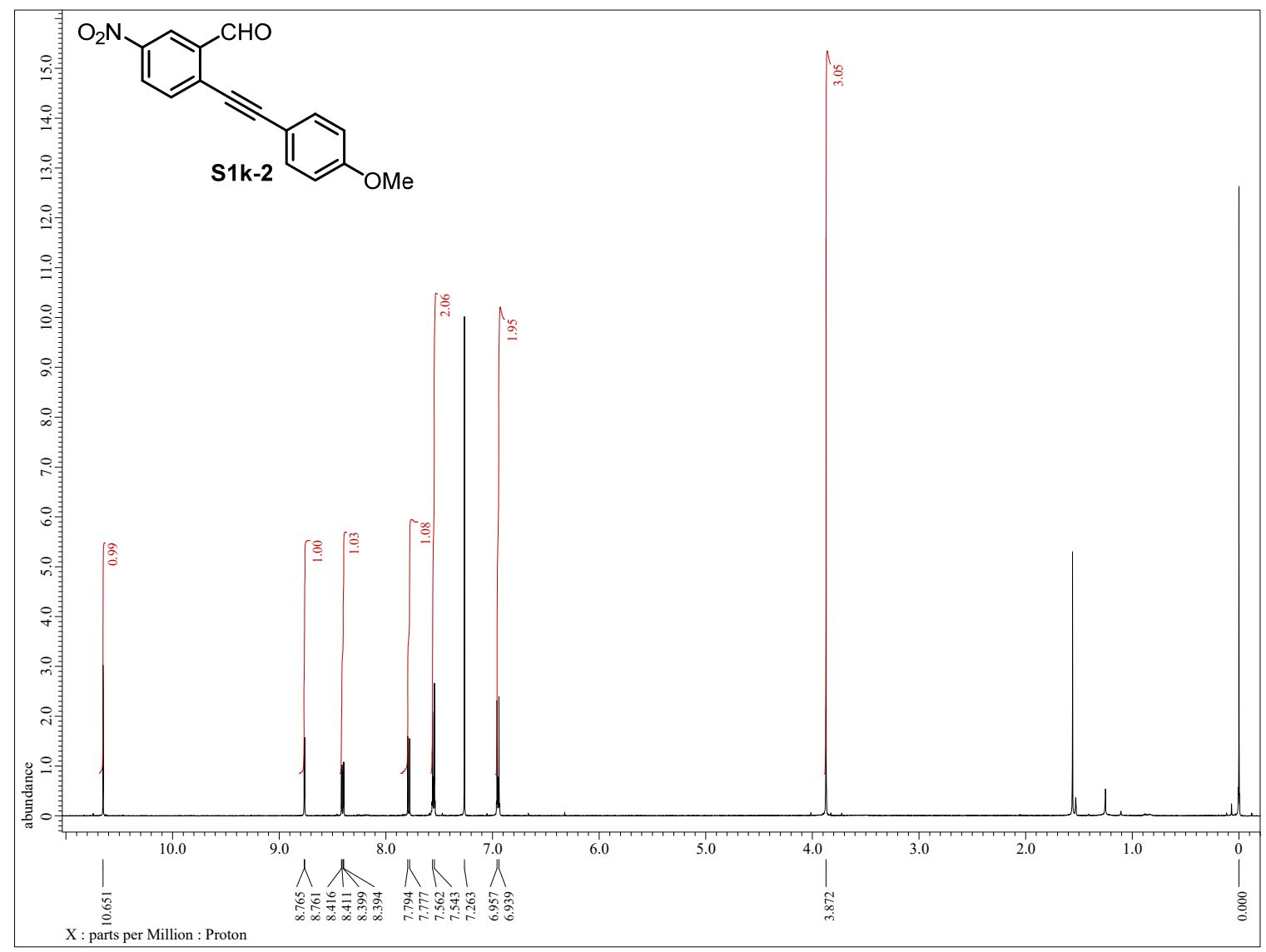

${ }^{1} \mathrm{H}$ NMR (400 MHz, $\mathrm{CDCl}_{3}$ ) of $\mathbf{1} \mathbf{k}$

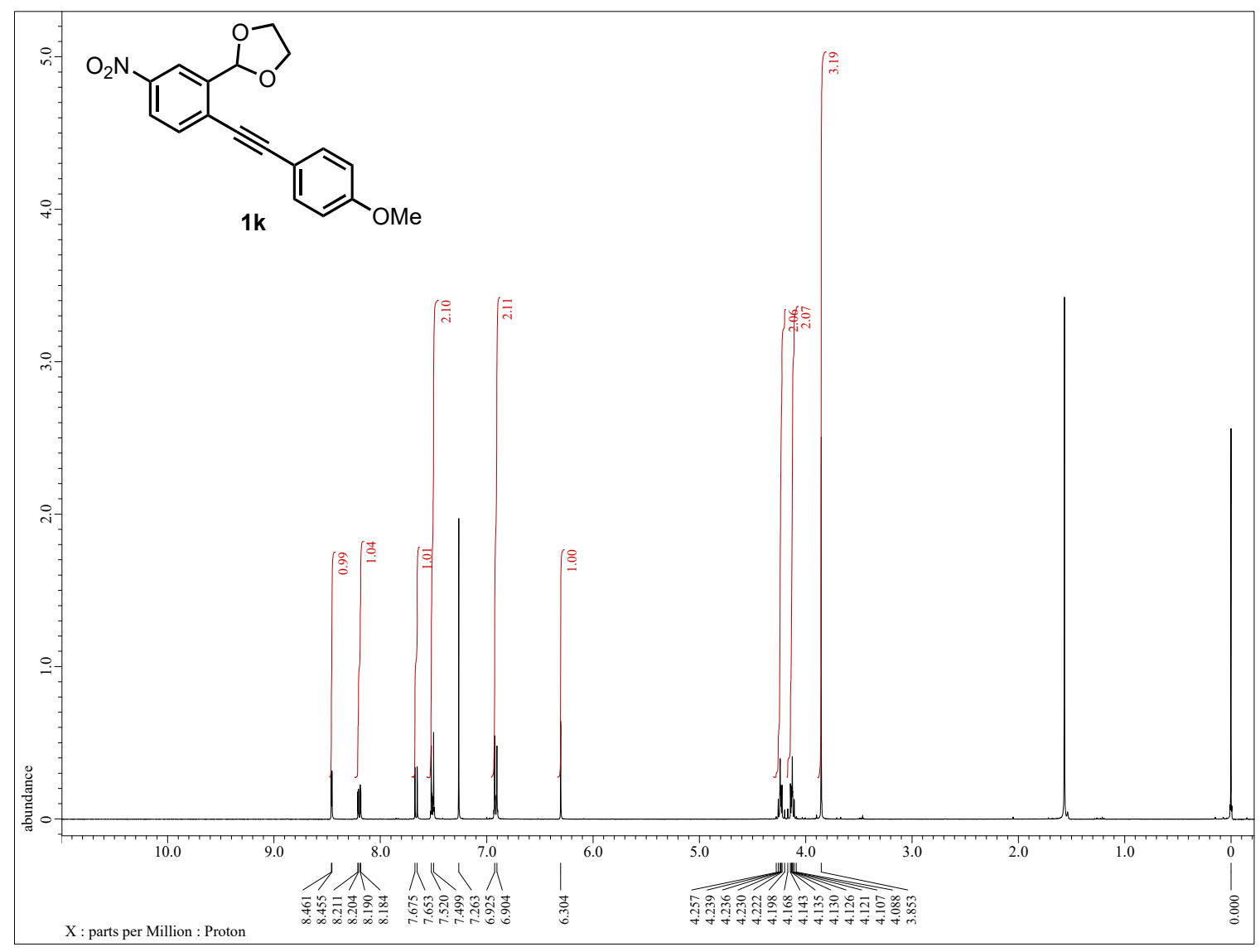

S50 
${ }^{13} \mathrm{C}$ NMR $\left(100 \mathrm{MHz}, \mathrm{CDCl}_{3}\right)$ of $\mathbf{1 k}$

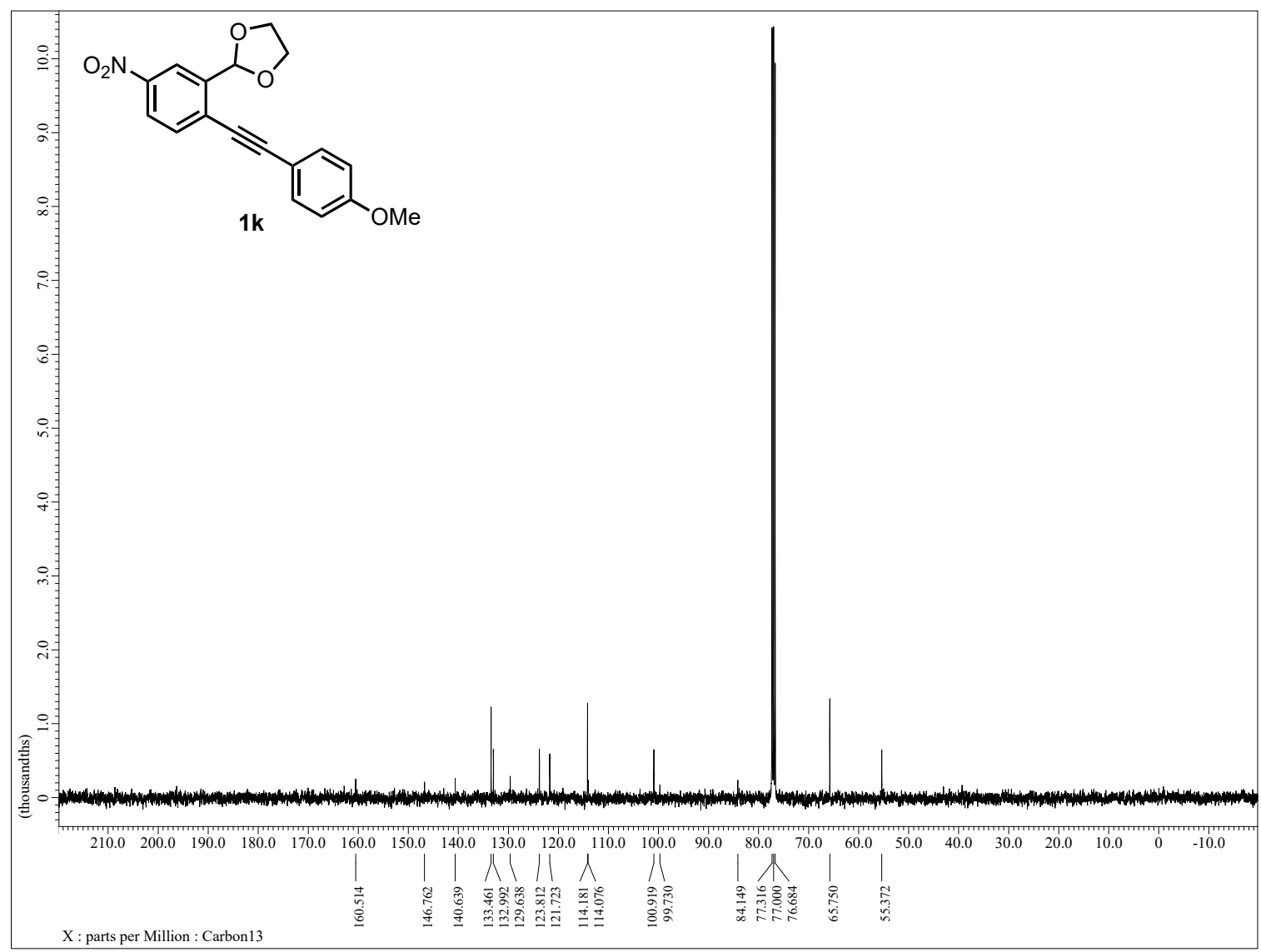

${ }^{1} \mathrm{H}$ NMR (400 MHz, $\mathrm{CDCl}_{3}$ ) of S1l-1

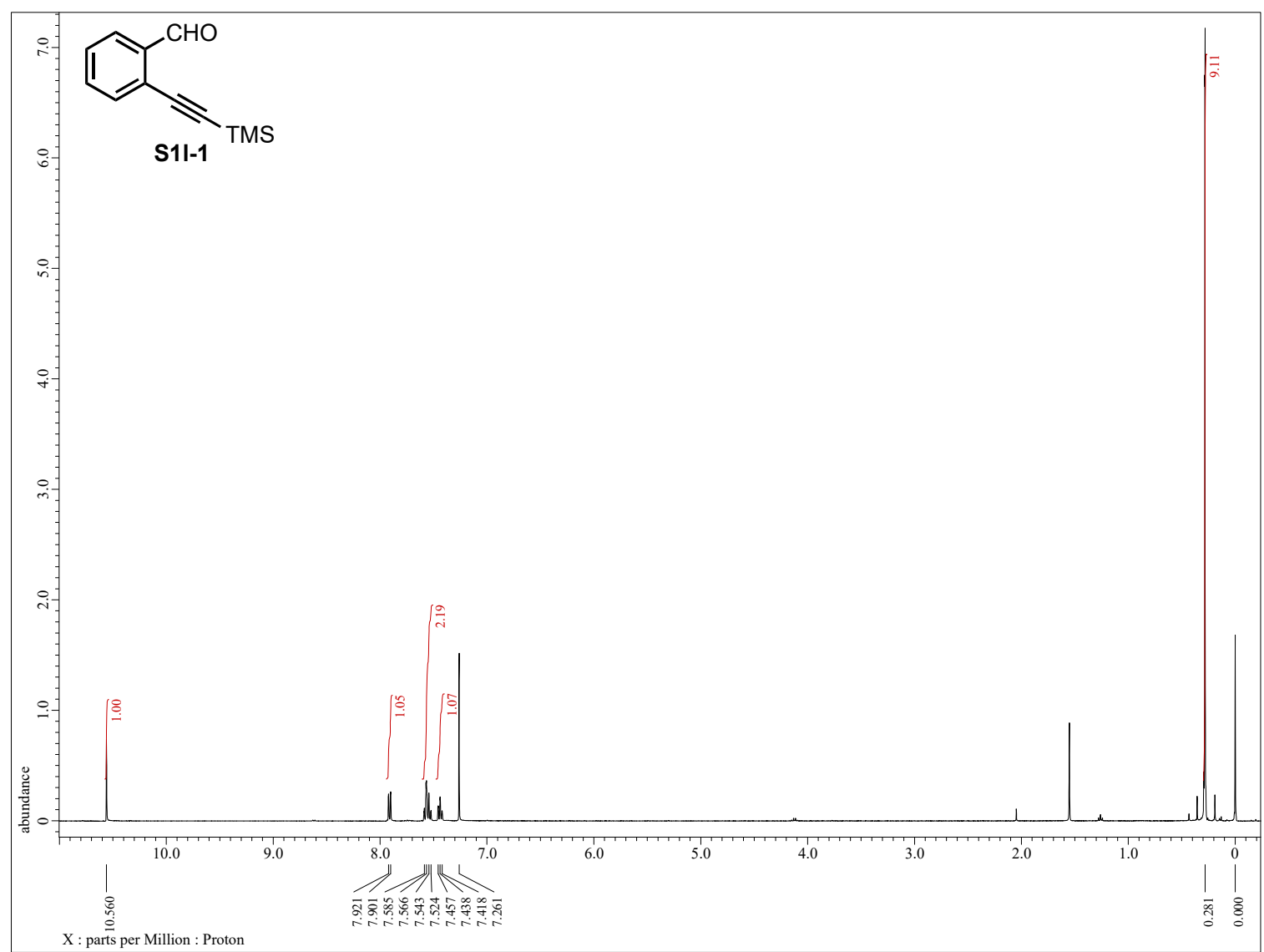


${ }^{1} \mathrm{H}$ NMR (400 MHz, $\mathrm{CDCl}_{3}$ ) of $\mathbf{S 1 1 - 2}$

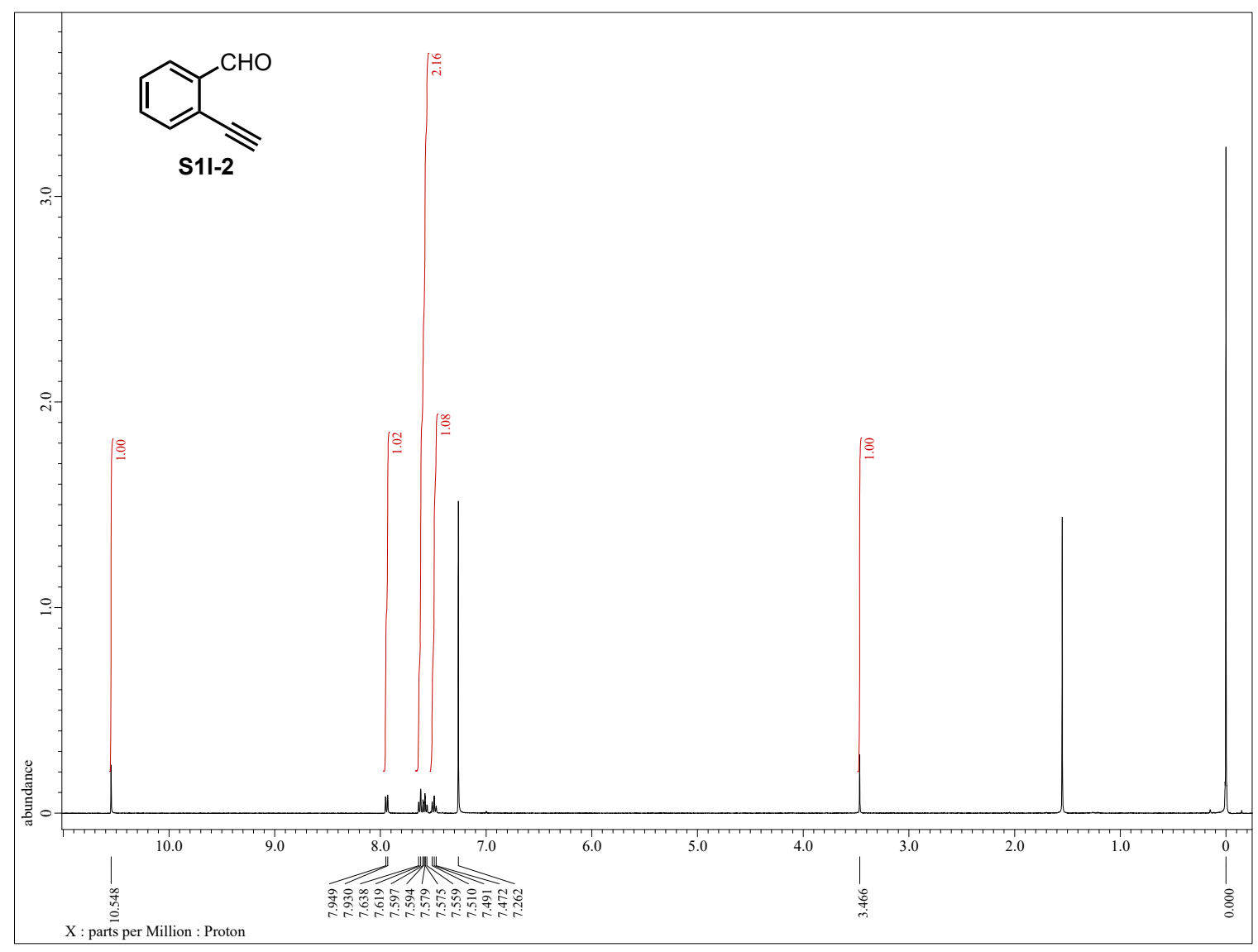

${ }^{1} \mathrm{H}$ NMR (400 MHz, $\mathrm{CDCl}_{3}$ ) of $\mathbf{S 1 1 - 3}$

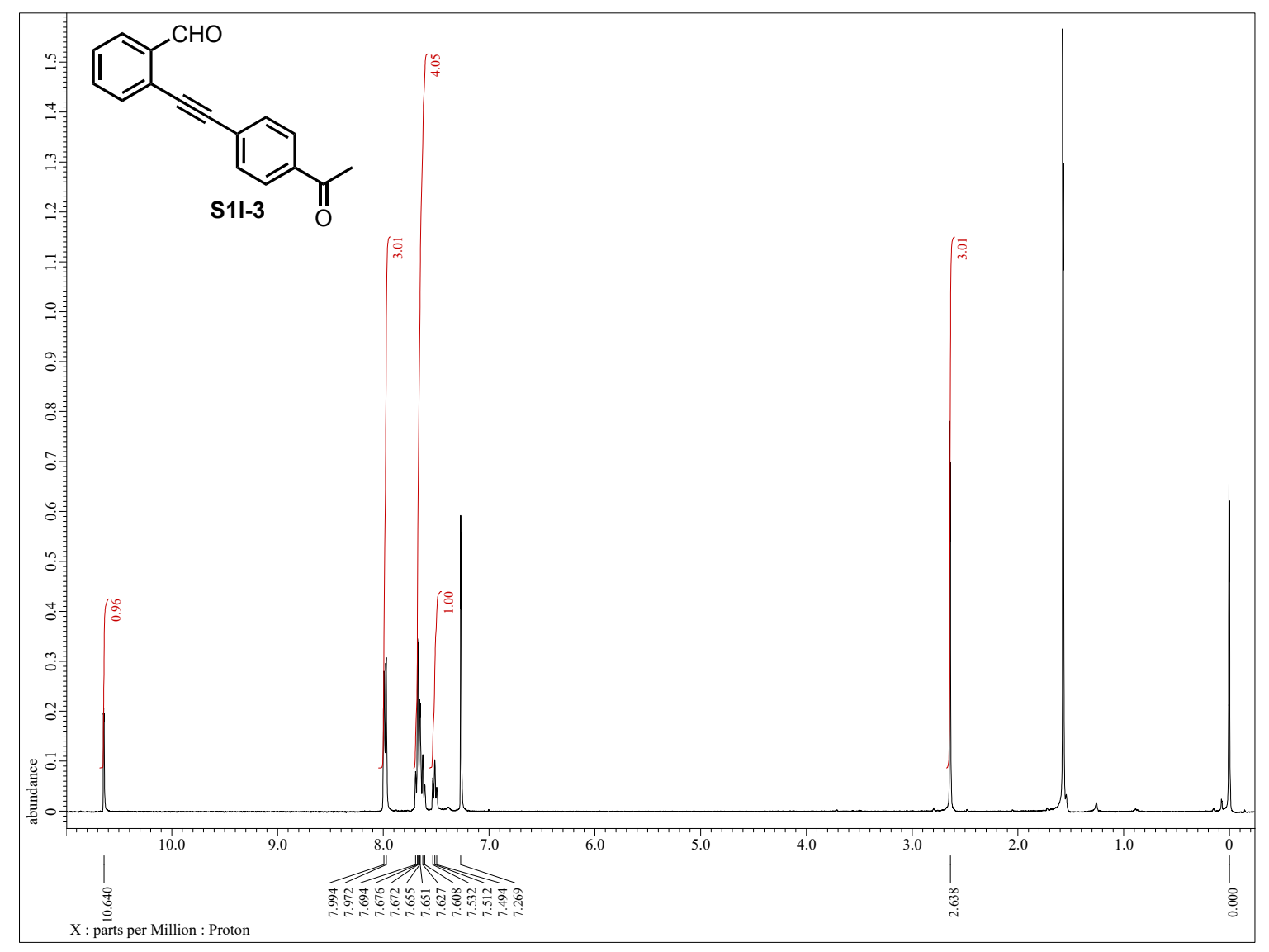

S52 
${ }^{1} \mathrm{H}$ NMR (400 MHz, $\mathrm{CDCl}_{3}$ ) of $\mathbf{1 I}$

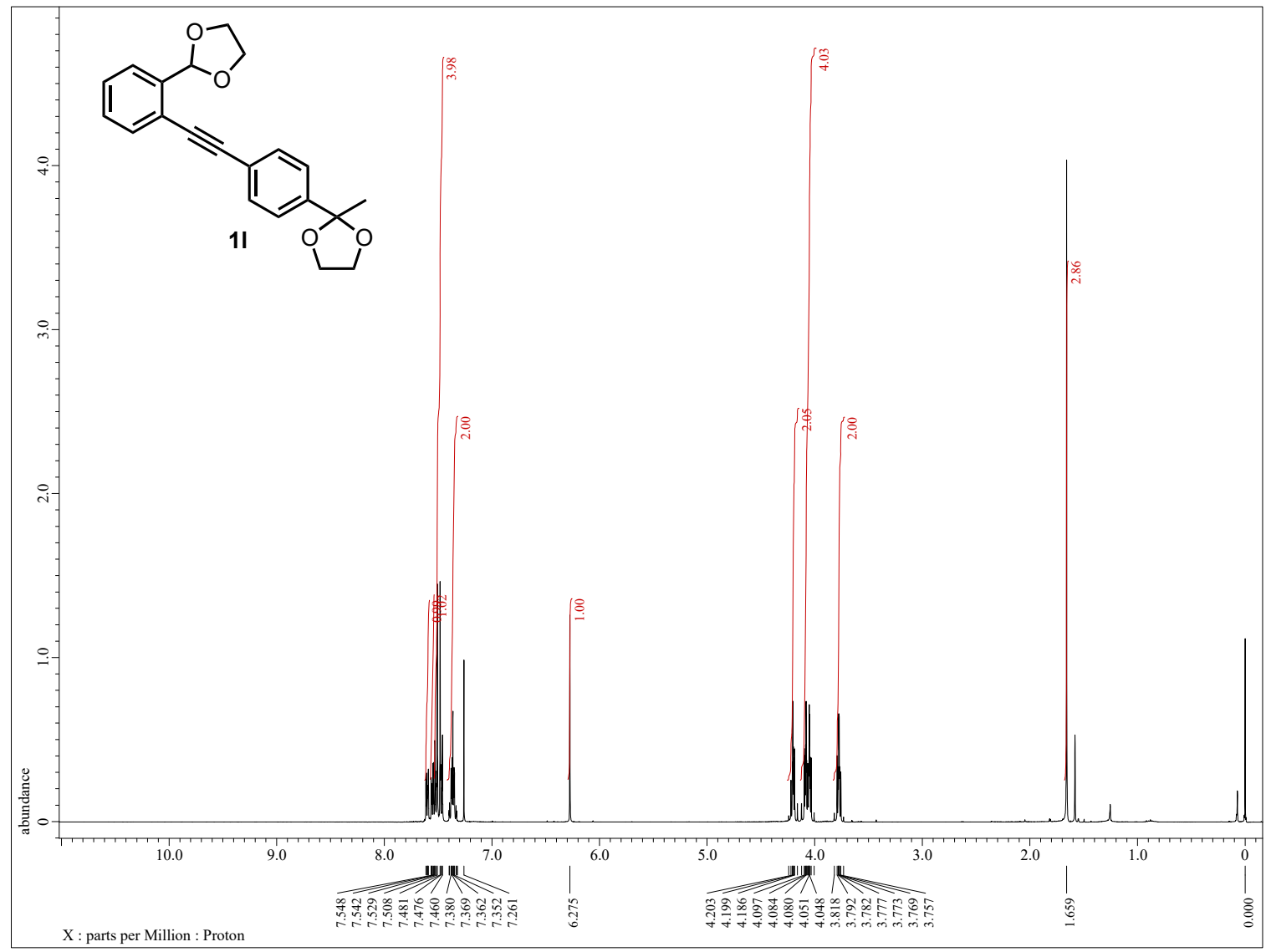

${ }^{13} \mathrm{C}$ NMR $\left(100 \mathrm{MHz}, \mathrm{CDCl}_{3}\right)$ of $\mathbf{1 l}$

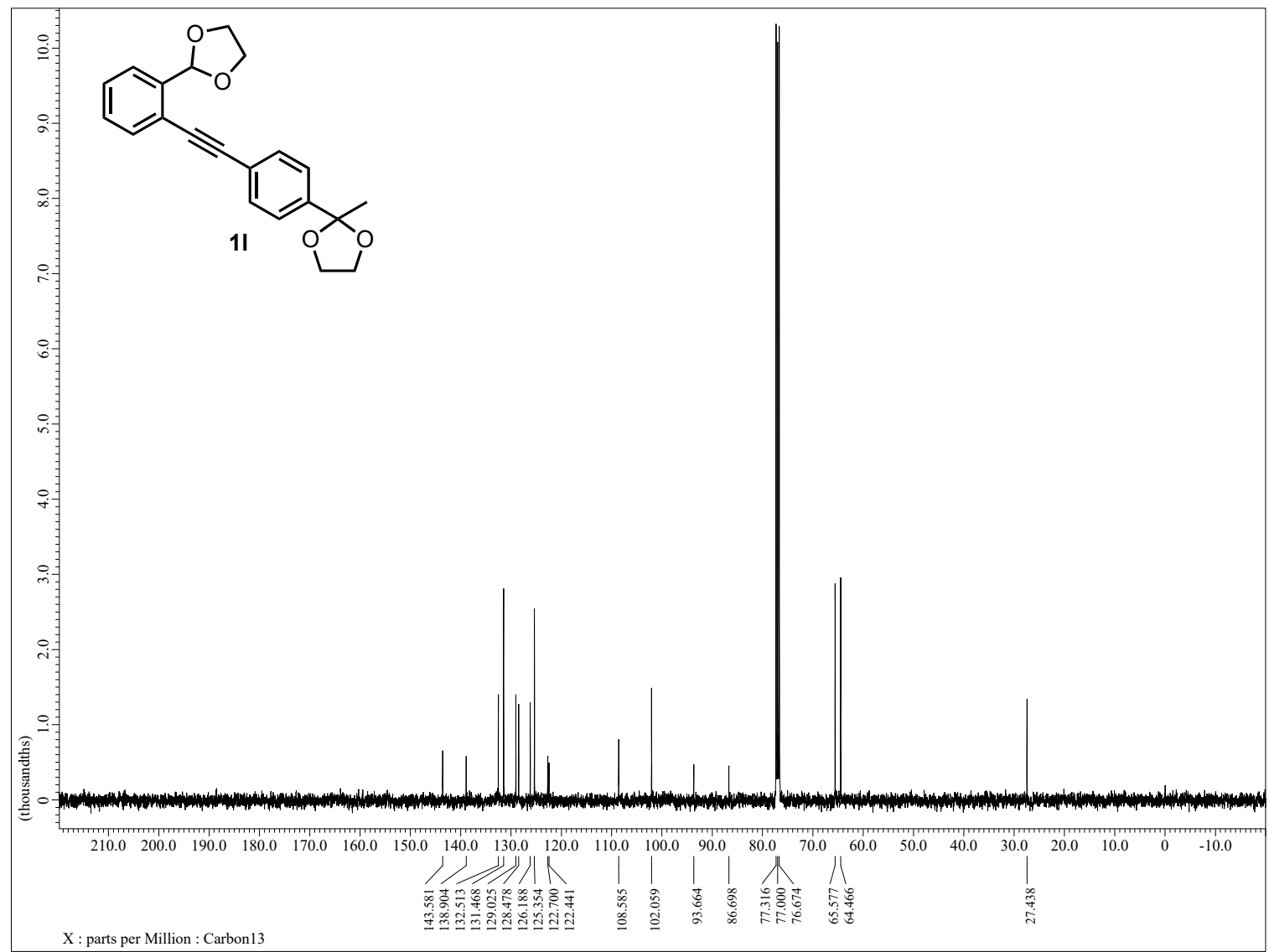


${ }^{1} \mathrm{H}$ NMR $\left(400 \mathrm{MHz}, \mathrm{CDCl}_{3}\right)$ of $\mathbf{S 1 m}$

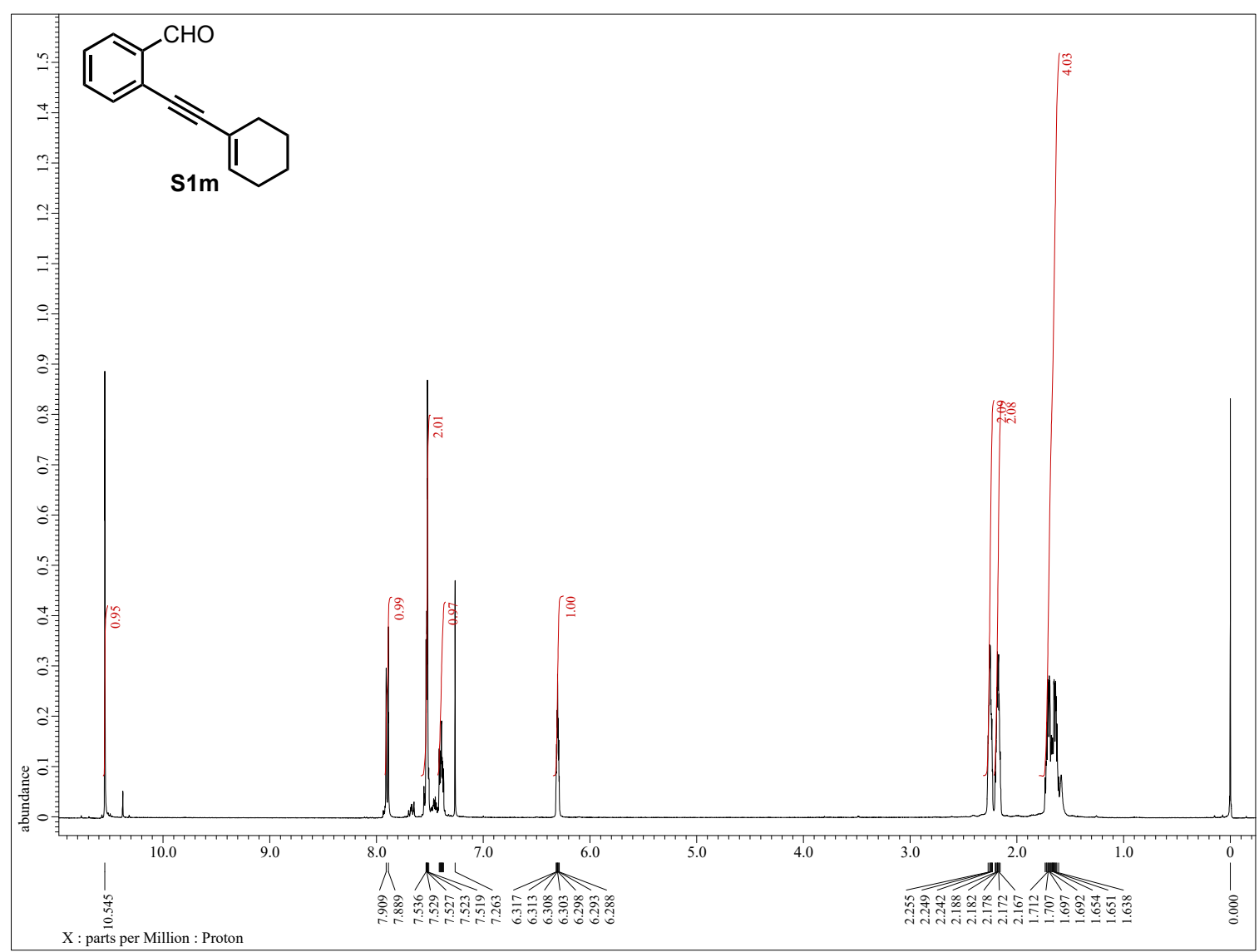

${ }^{1} \mathrm{H}$ NMR (400 MHz, $\mathrm{CDCl}_{3}$ ) of $\mathbf{1 m}$

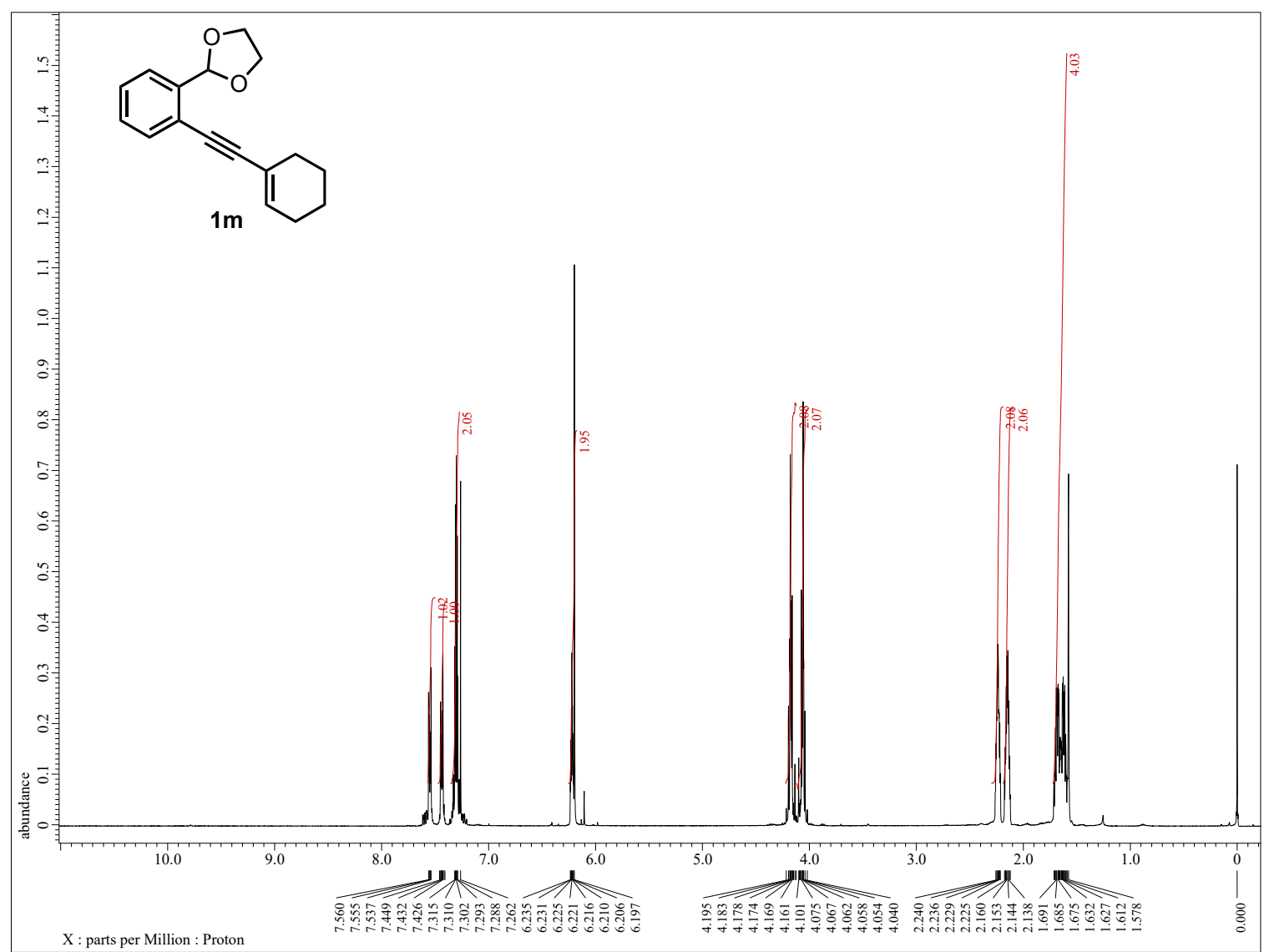


${ }^{13} \mathrm{C}$ NMR $\left(100 \mathrm{MHz}, \mathrm{CDCl}_{3}\right)$ of $\mathbf{1 m}$

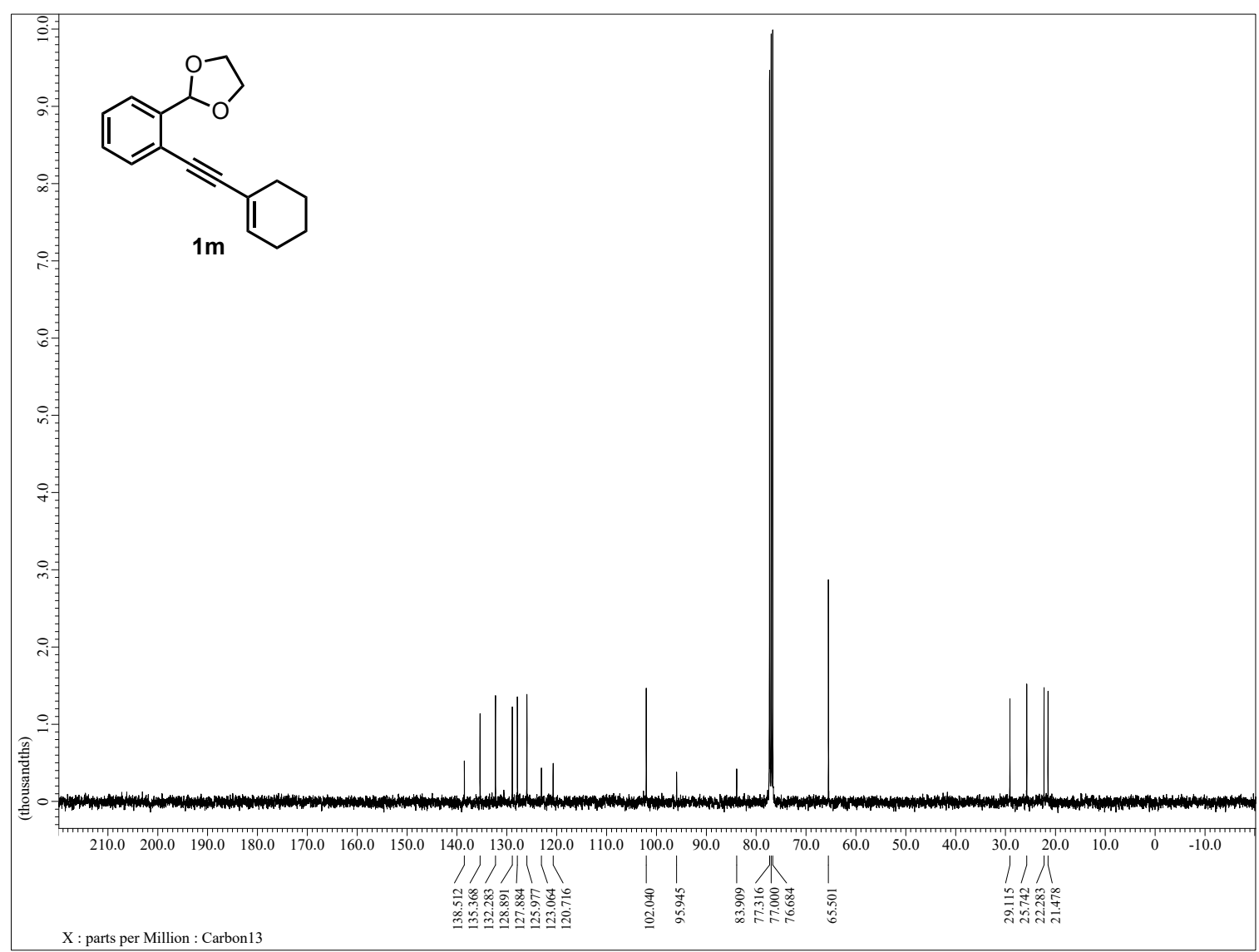

${ }^{1} \mathrm{H}$ NMR (400 MHz, $\mathrm{CDCl}_{3}$ ) of $\mathbf{S 1 n}$

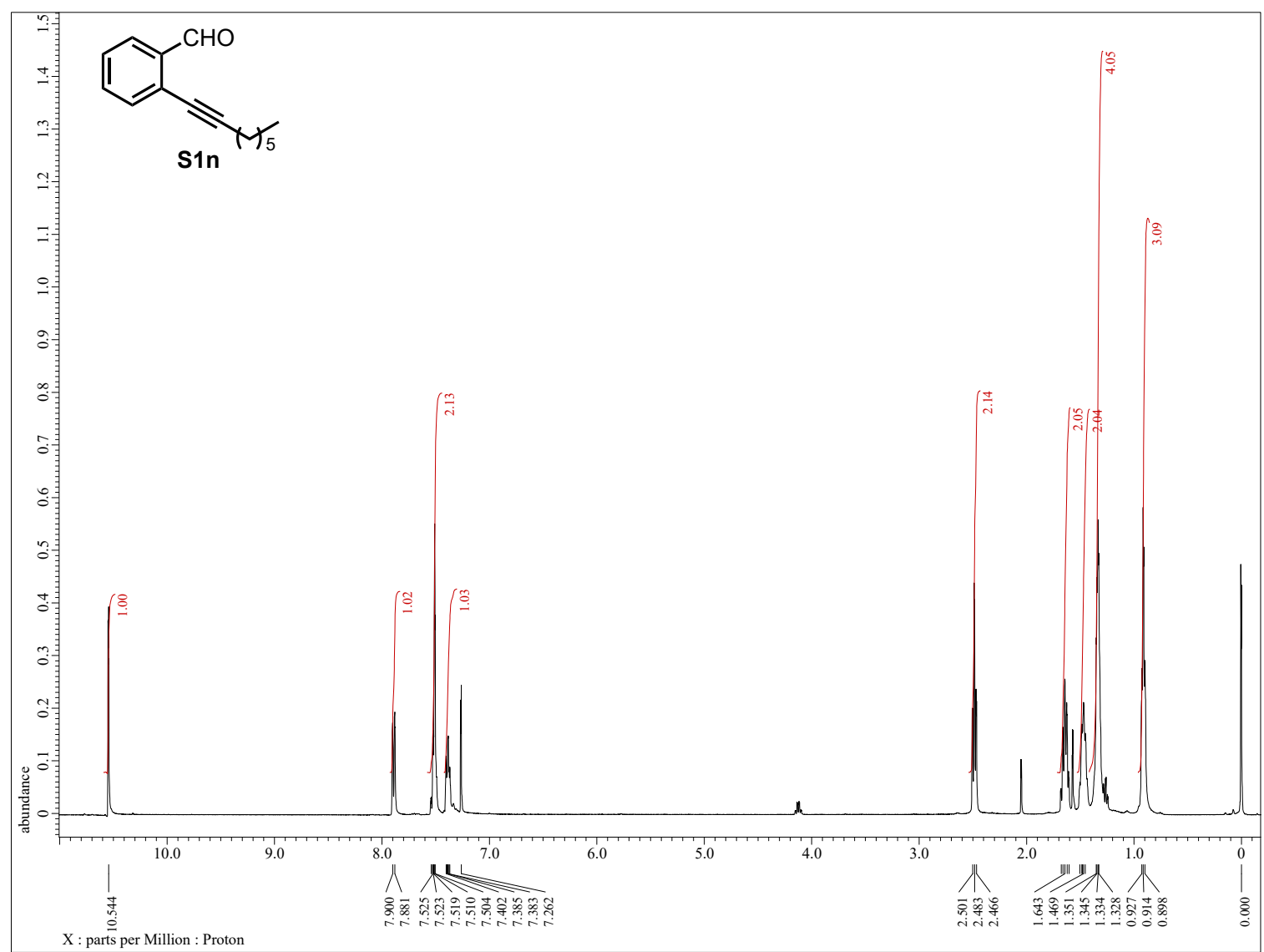


${ }^{1} \mathrm{H}$ NMR (400 MHz, $\mathrm{CDCl}_{3}$ ) of $\mathbf{1 n}$

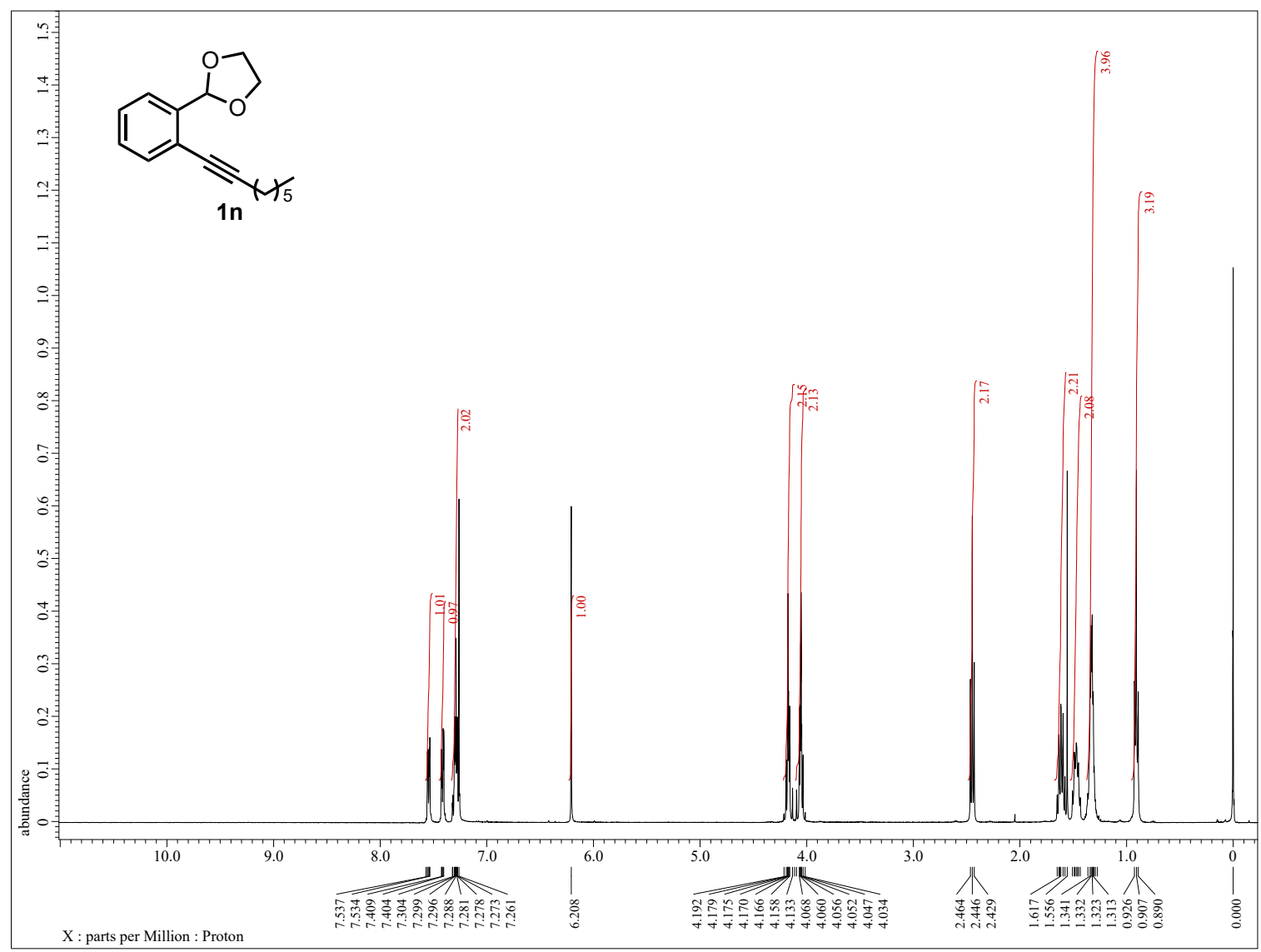

${ }^{13} \mathrm{C}$ NMR $\left(100 \mathrm{MHz}, \mathrm{CDCl}_{3}\right)$ of $\mathbf{1 n}$

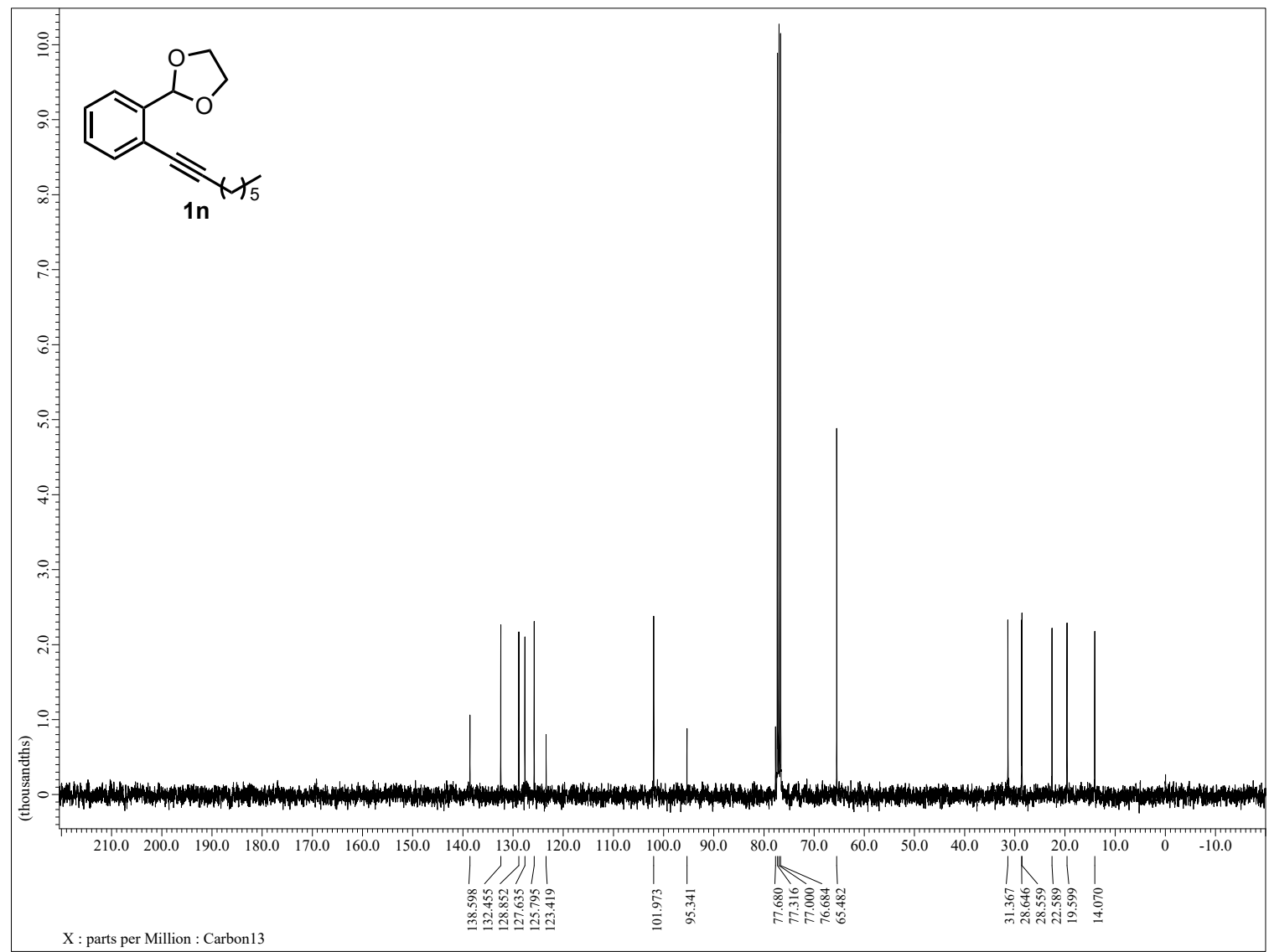


${ }^{1} \mathrm{H}$ NMR (500 MHz, $\mathrm{CDCl}_{3}$ ) of S1o-1

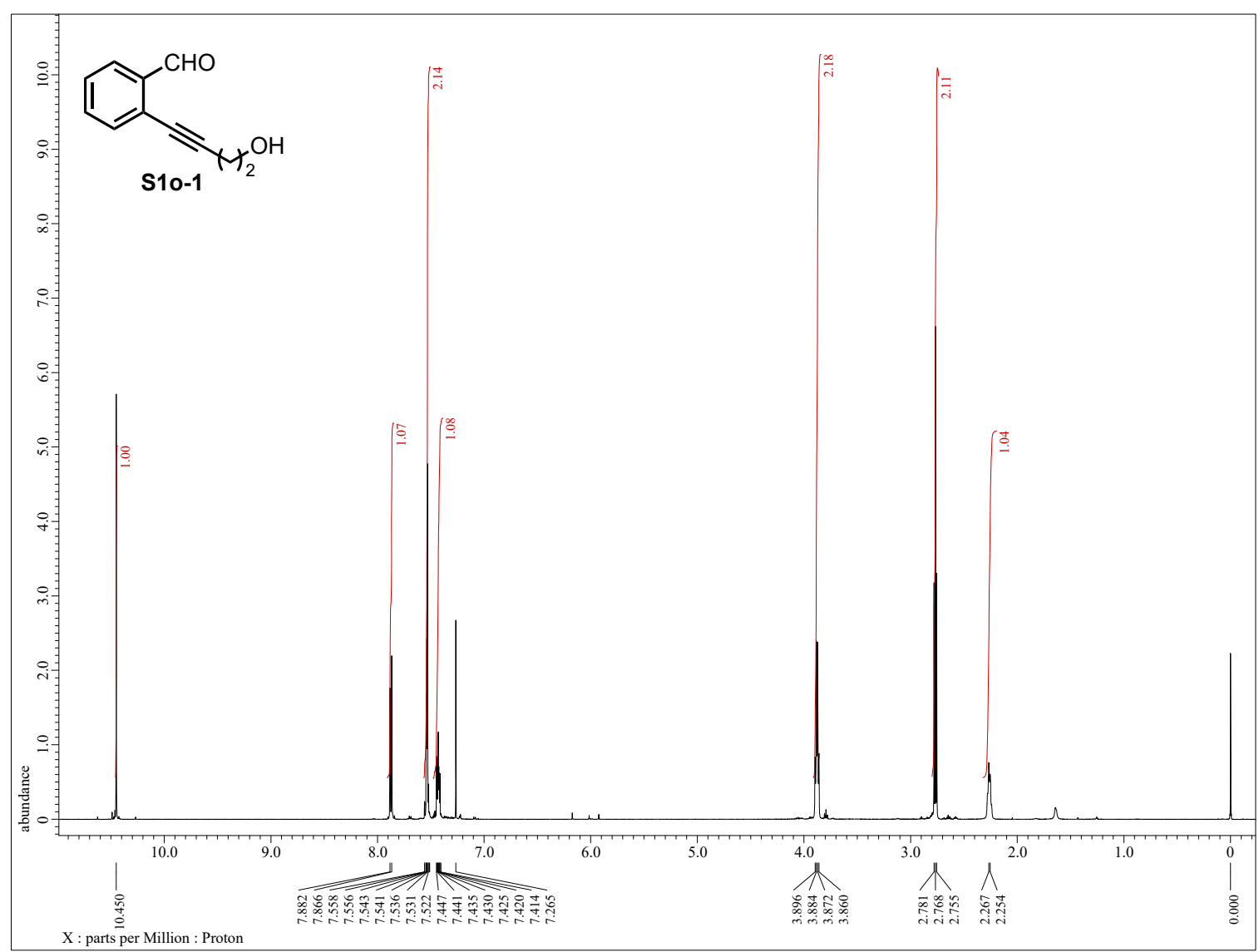

${ }^{1} \mathrm{H}$ NMR (400 MHz, $\mathrm{CDCl}_{3}$ ) of S1o-2

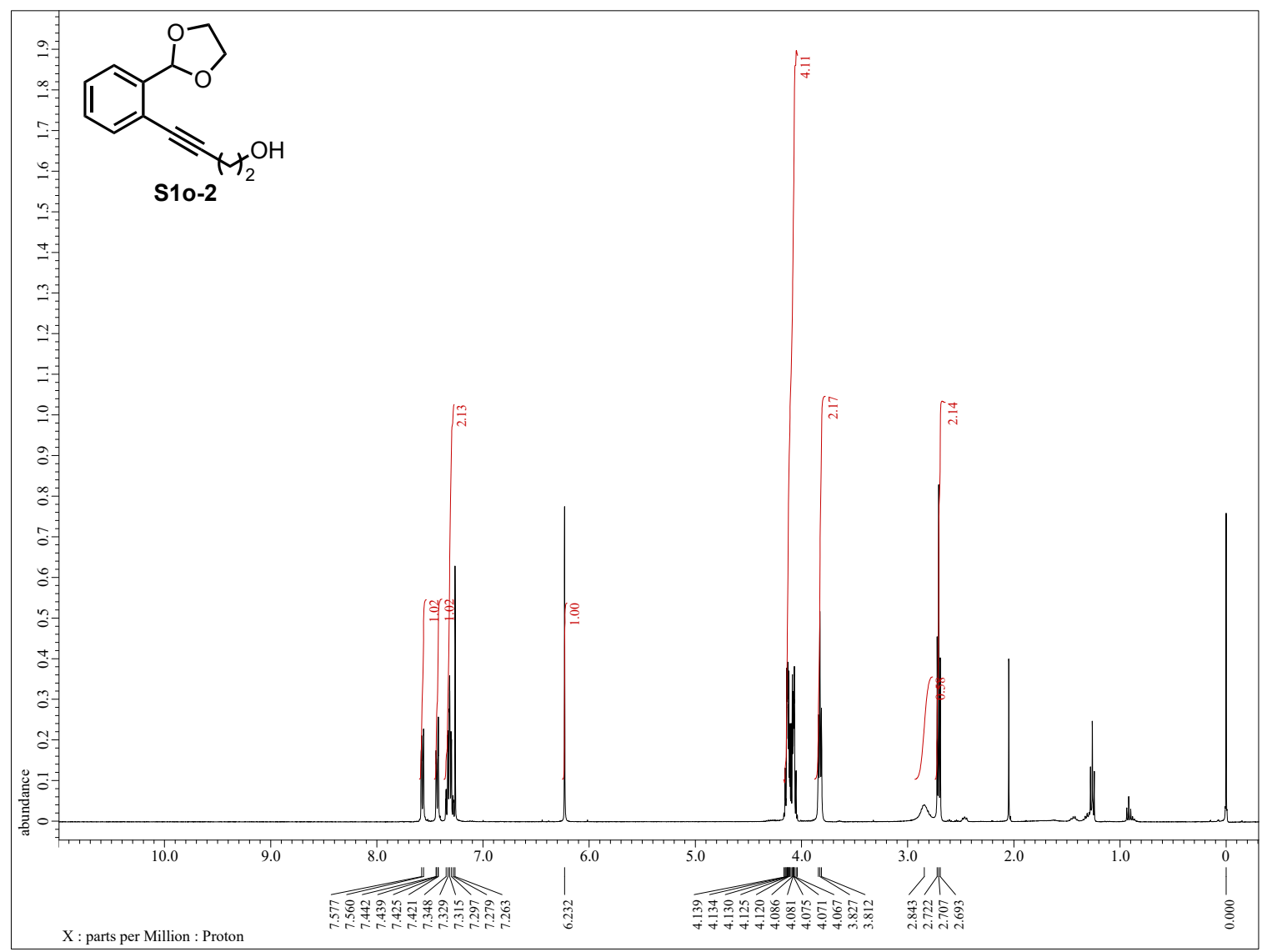


${ }^{1} \mathrm{H}$ NMR $\left(100 \mathrm{MHz}, \mathrm{CDCl}_{3}\right)$ of $\mathbf{1 o}$

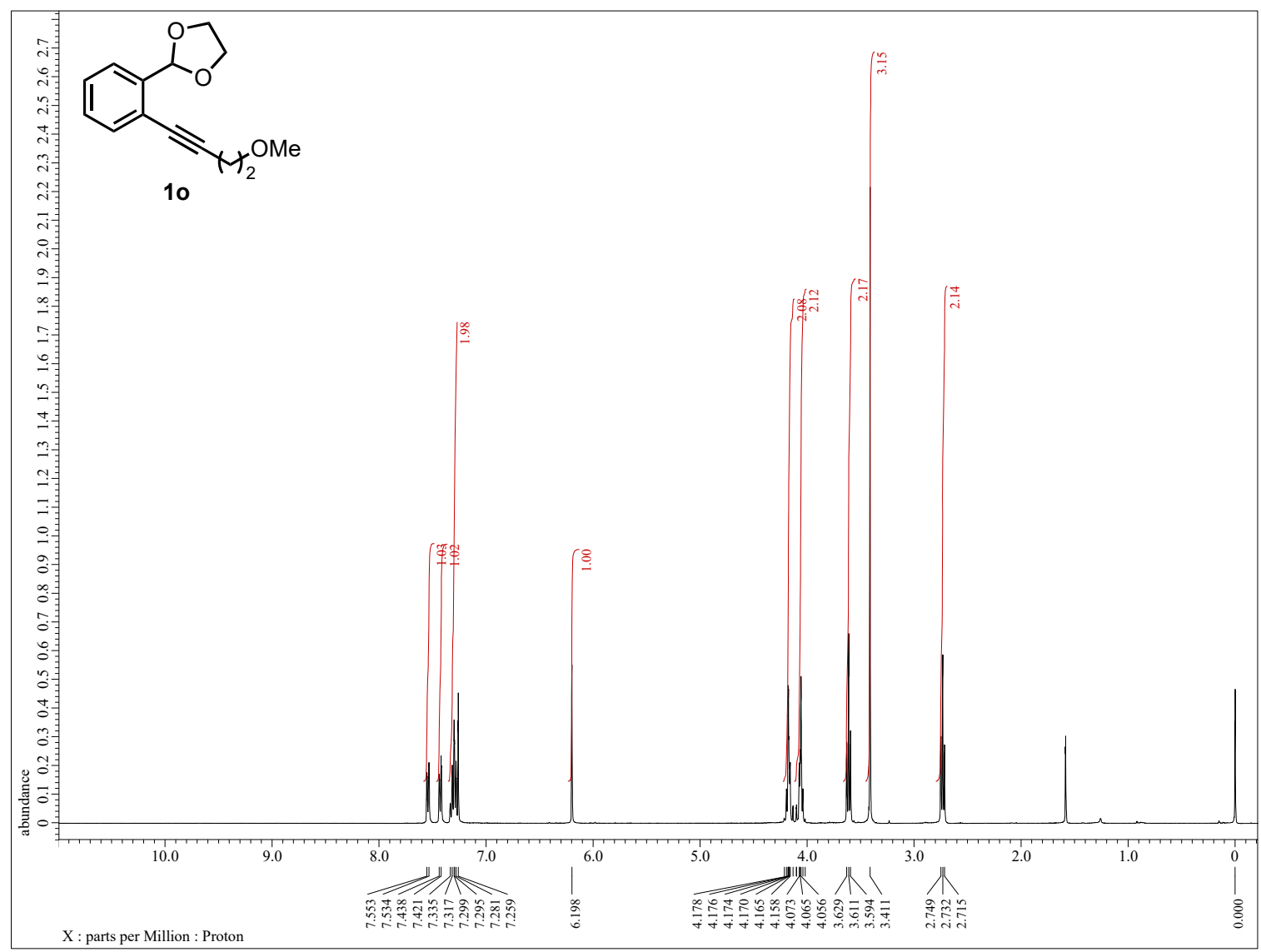

${ }^{13} \mathrm{C}$ NMR $\left(100 \mathrm{MHz}, \mathrm{CDCl}_{3}\right)$ of $\mathbf{1 o}$

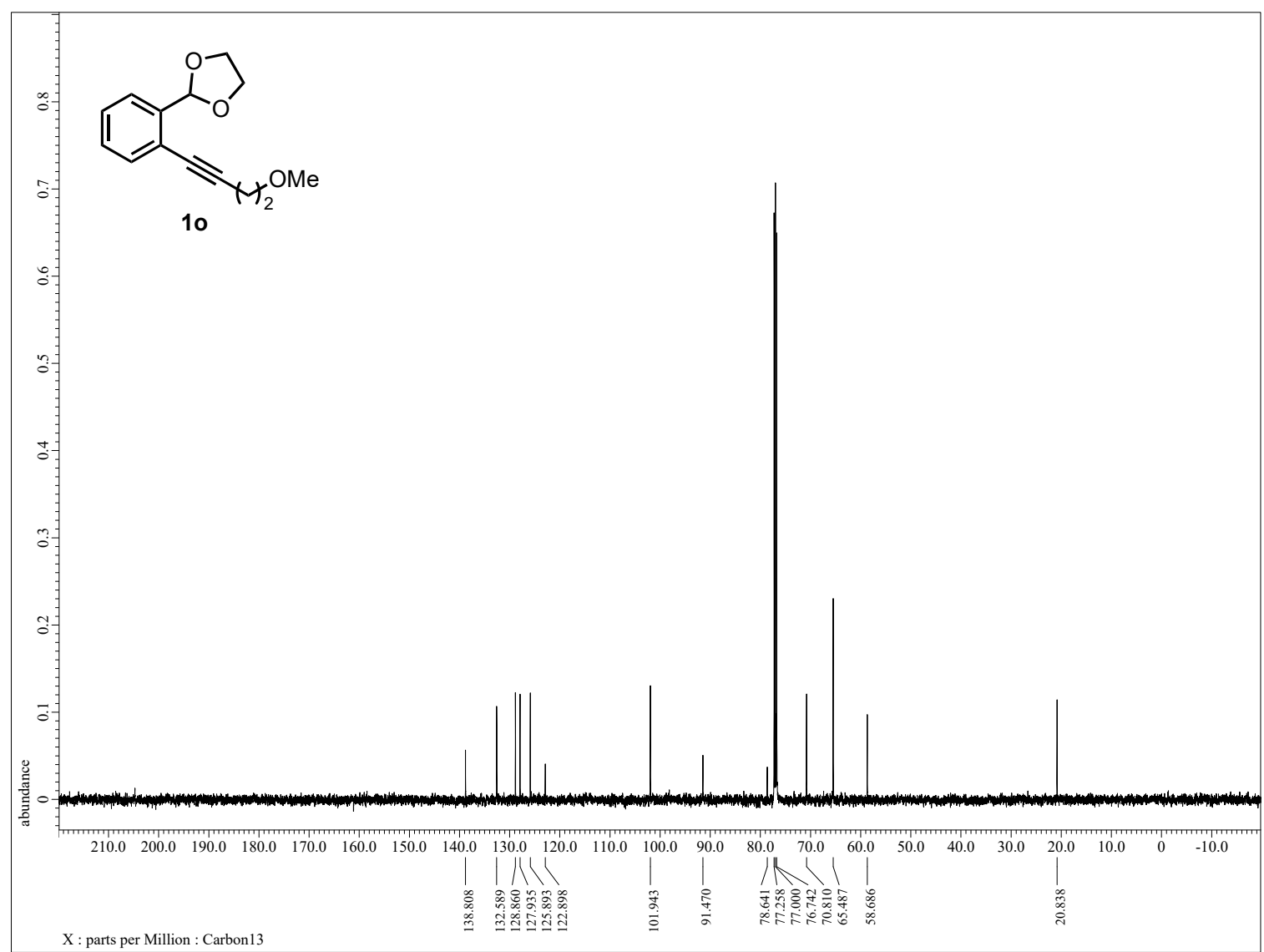


${ }^{1} \mathrm{H}$ NMR (400 MHz, $\mathrm{CDCl}_{3}$ ) of $\mathbf{S 1 p - 1}$

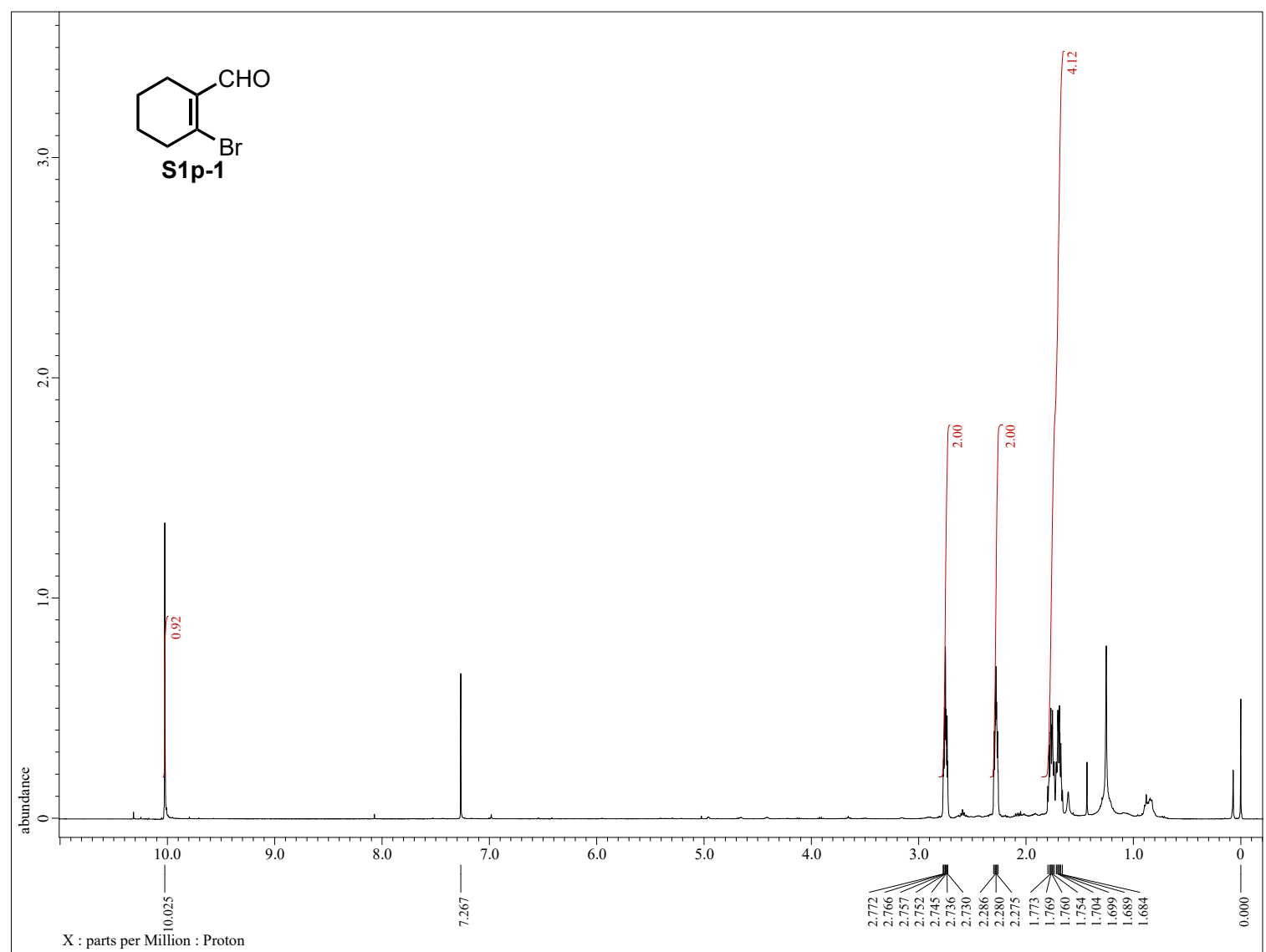

${ }^{1} \mathrm{H}$ NMR (400 MHz, $\mathrm{CDCl}_{3}$ ) of $\mathbf{S 1 p - 2}$

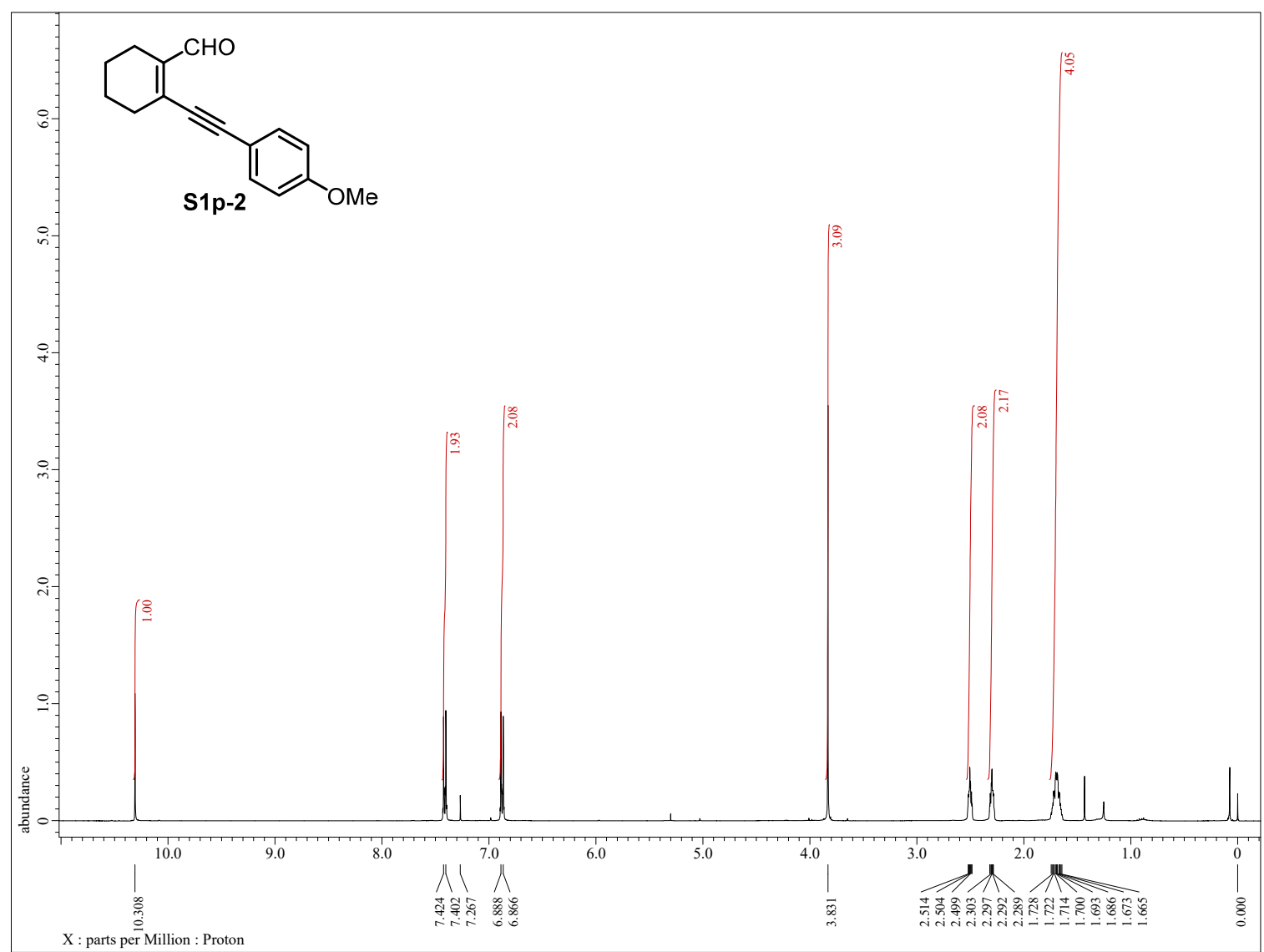


${ }^{1} \mathrm{H}$ NMR (500 MHz, $\mathrm{CDCl}_{3}$ ) of $\mathbf{1 p}$

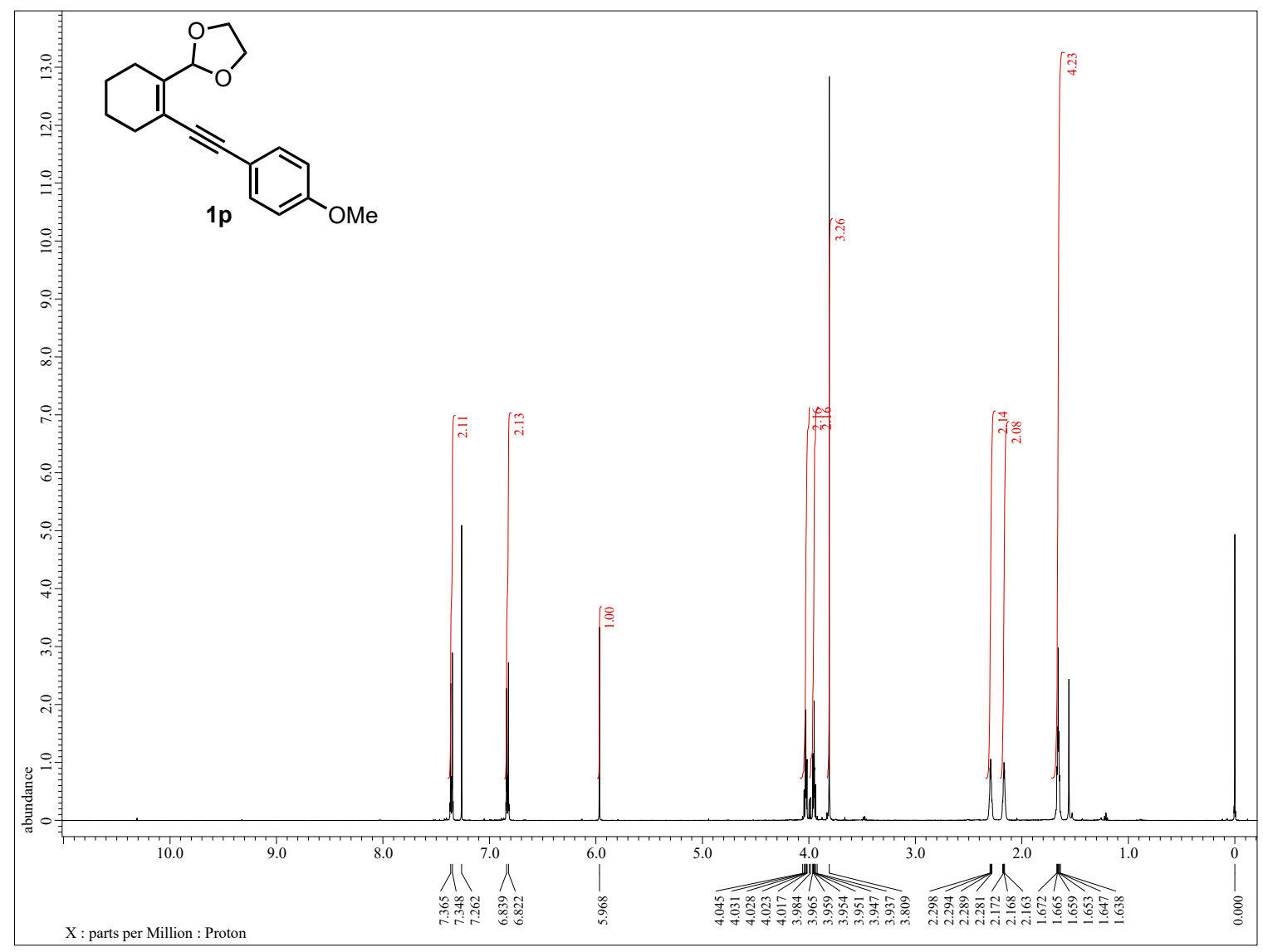

${ }^{13} \mathrm{C}$ NMR $\left(100 \mathrm{MHz}, \mathrm{CDCl}_{3}\right)$ of $\mathbf{1 p}$

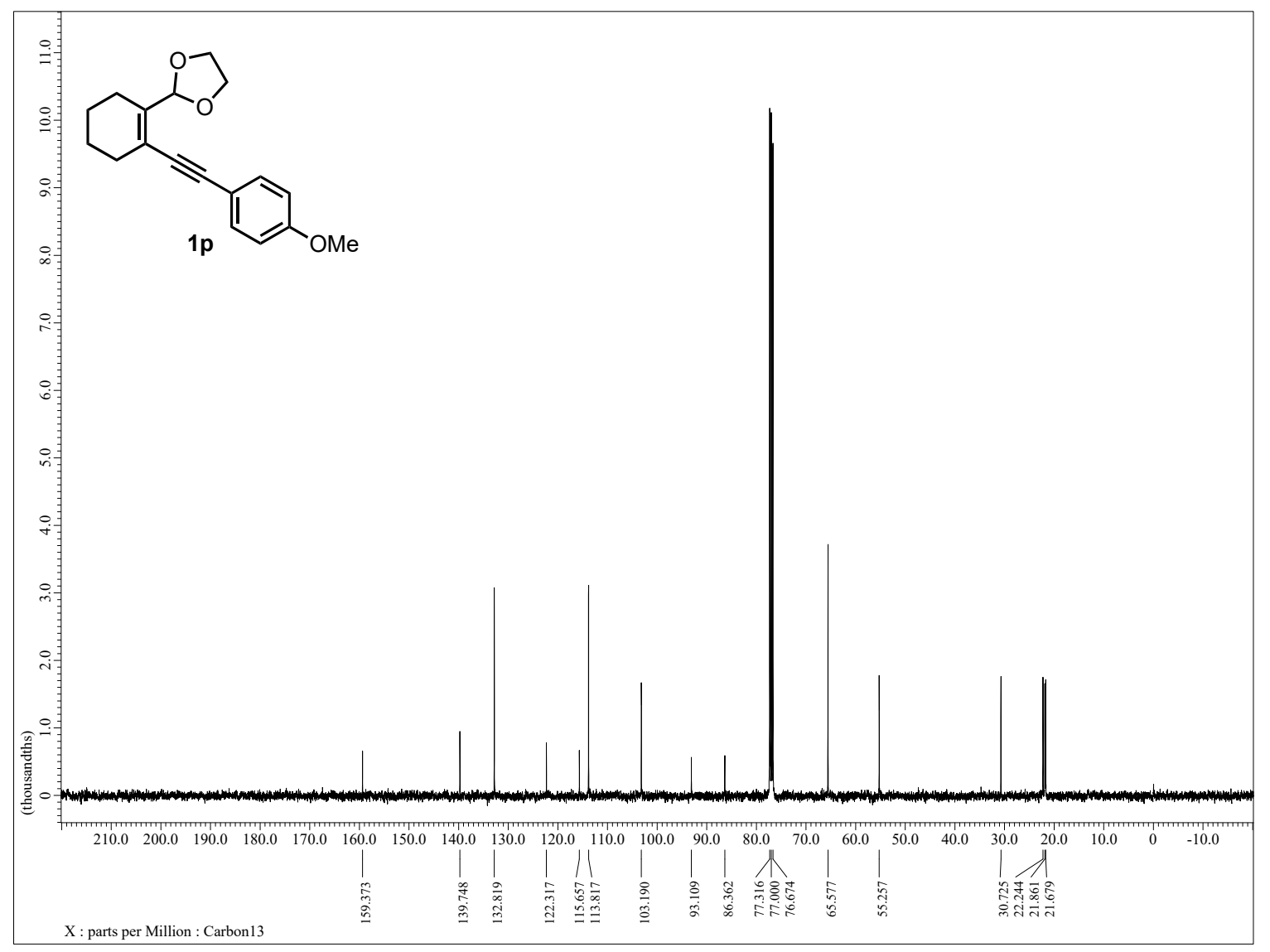

S60 
${ }^{1} \mathrm{H}$ NMR (400 MHz, $\mathrm{CDCl}_{3}$ ) of $\mathbf{1 q}$

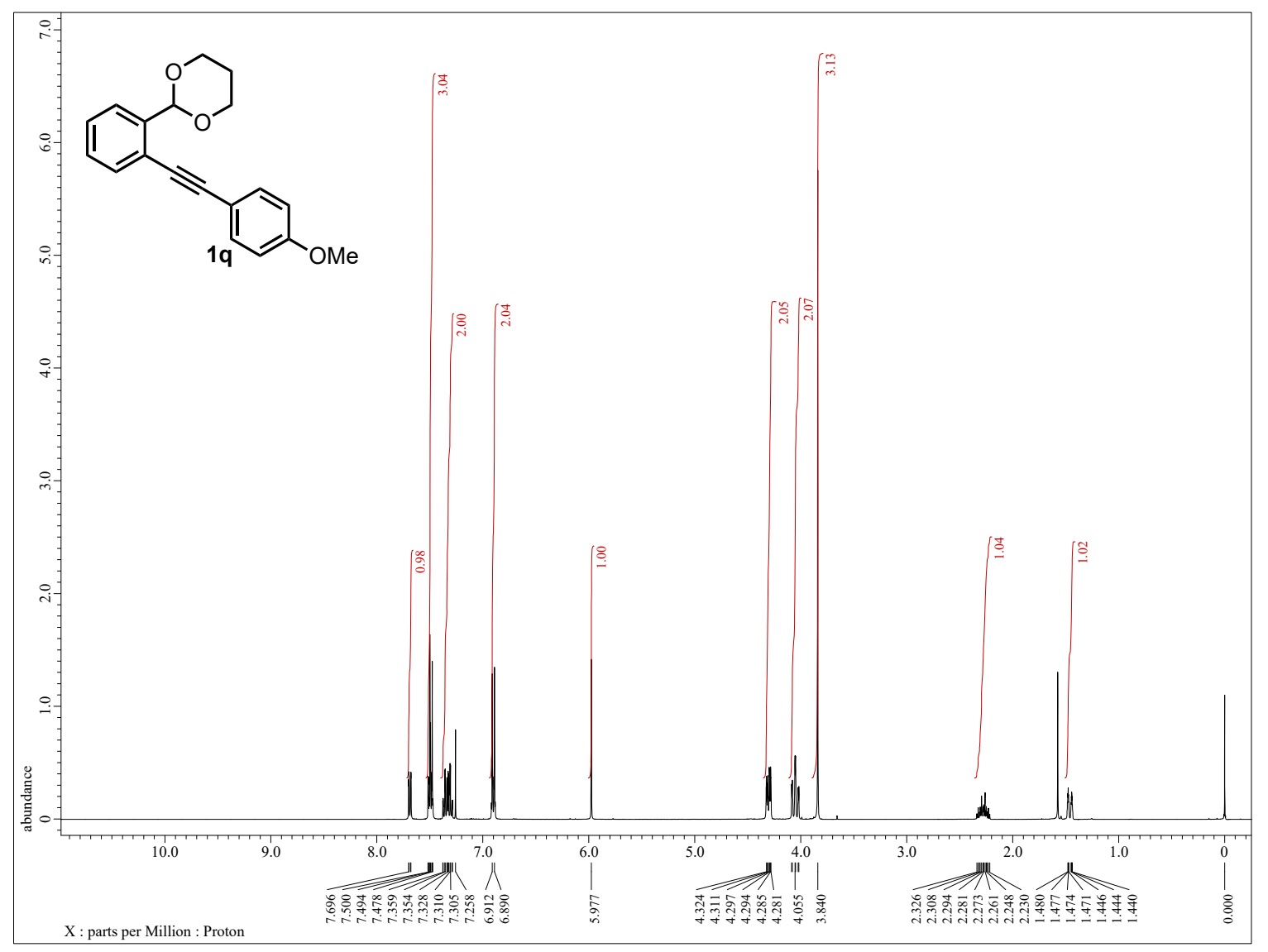

${ }^{13} \mathrm{C}$ NMR $\left(100 \mathrm{MHz}, \mathrm{CDCl}_{3}\right)$ of $\mathbf{1 p}$

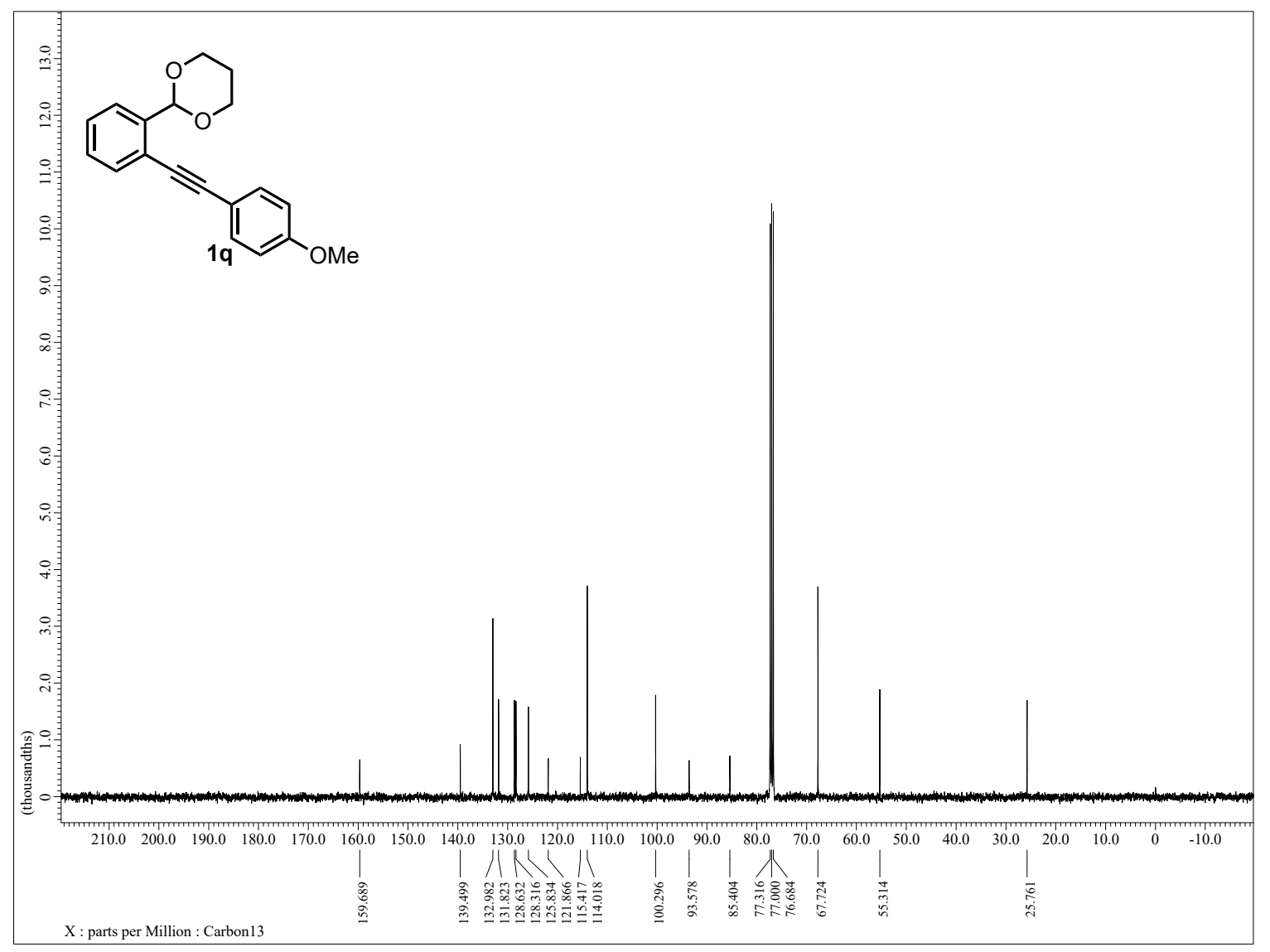

S61 
${ }^{1} \mathrm{H}$ NMR (400 MHz, $\mathrm{CDCl}_{3}$ ) of $\mathbf{1 r}$

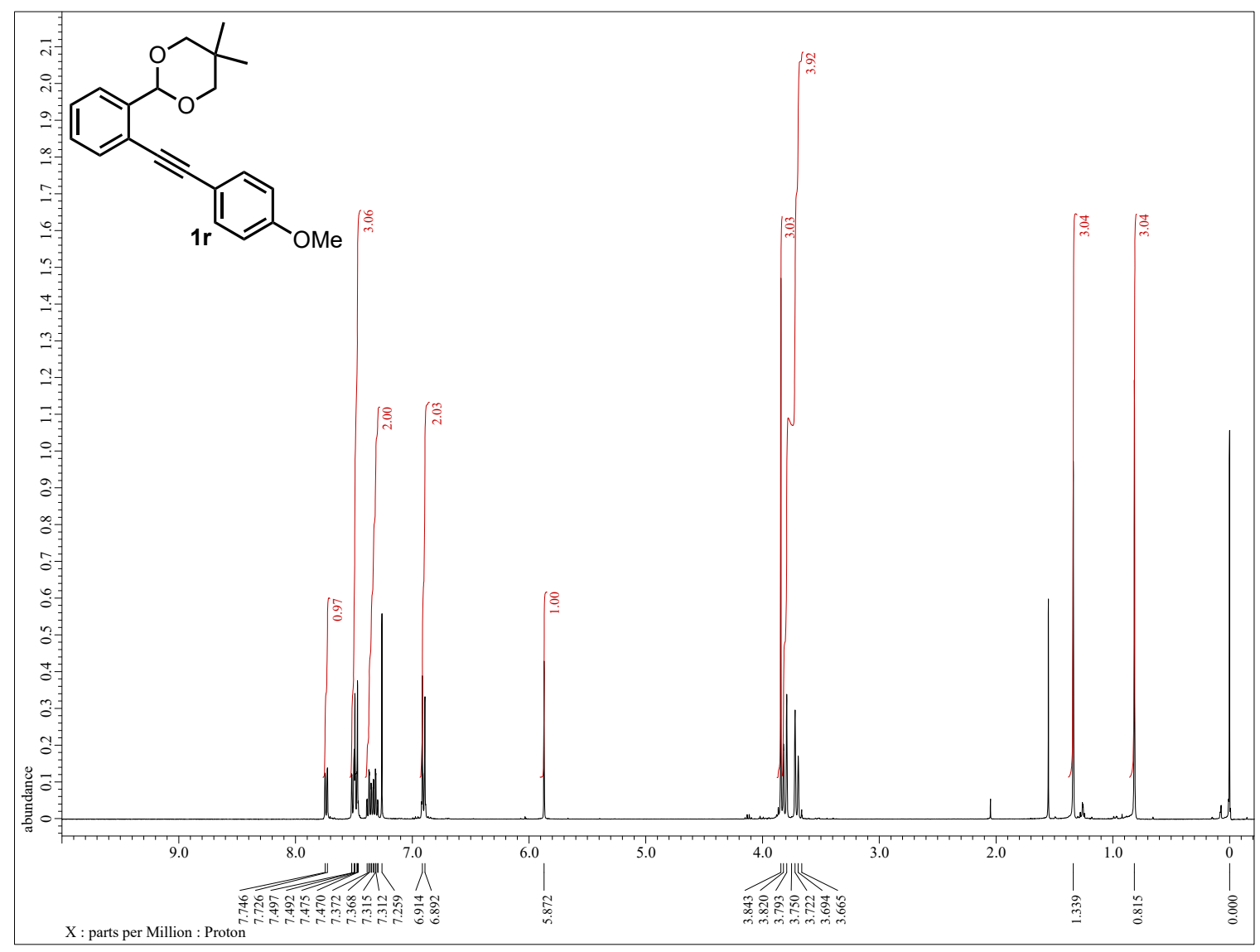

${ }^{13} \mathrm{C}$ NMR $\left(100 \mathrm{MHz}, \mathrm{CDCl}_{3}\right)$ of $\mathbf{1 r}$

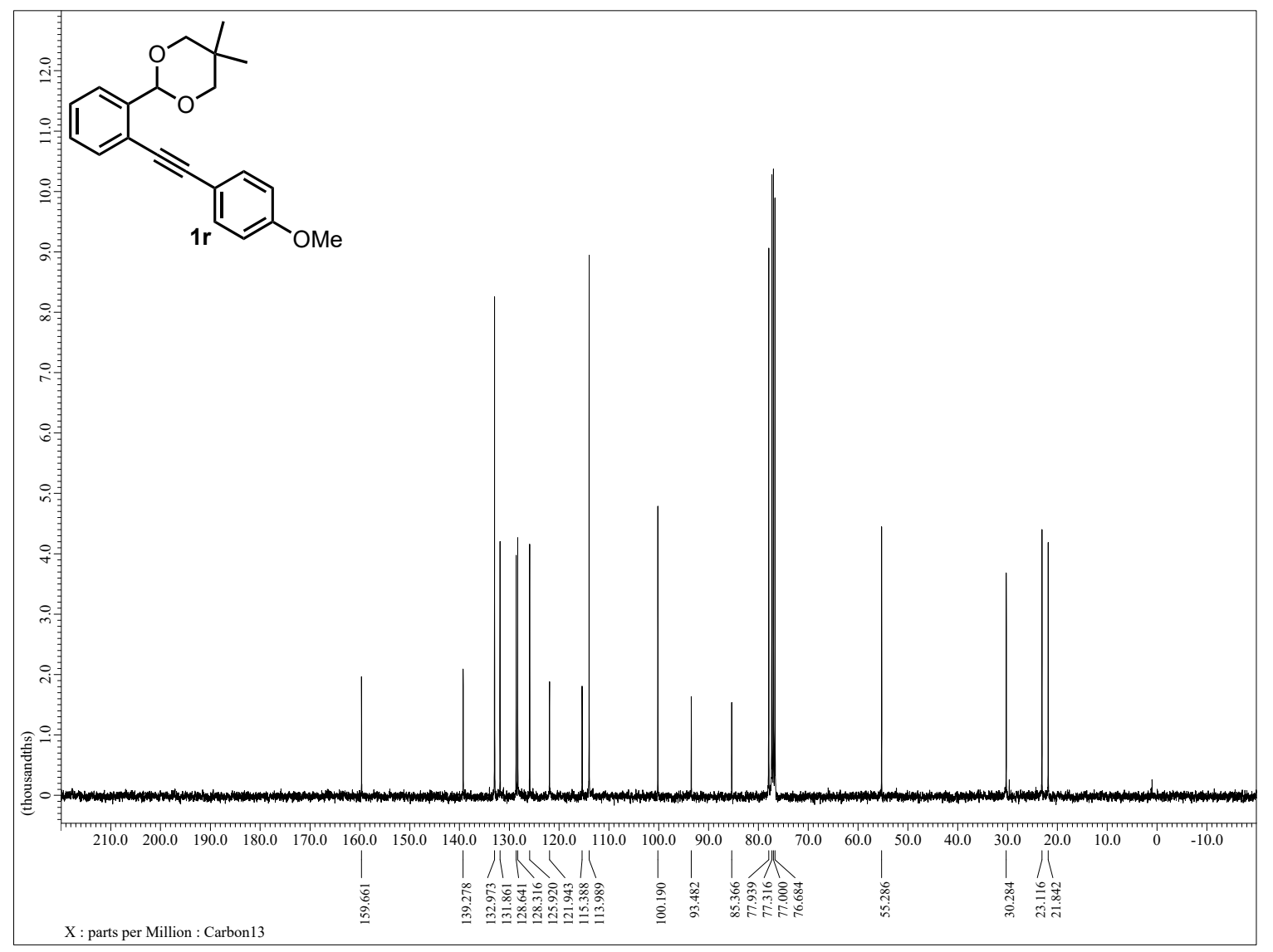

S62 
${ }^{1} \mathrm{H}$ NMR (400 MHz, $\mathrm{CDCl}_{3}$ ) of $\mathbf{1 s}$

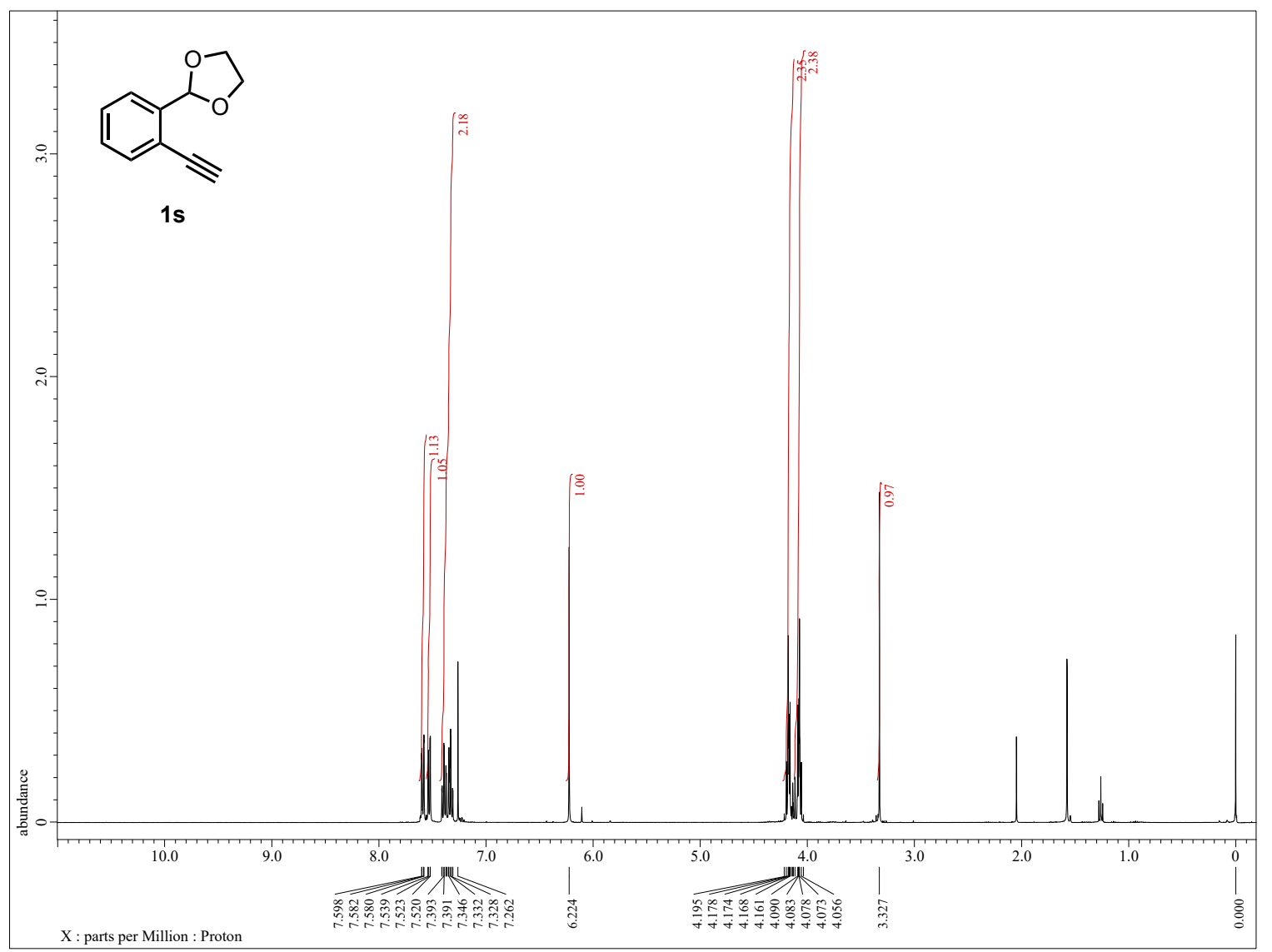

${ }^{1} \mathrm{H}$ NMR (400 MHz, $\mathrm{CDCl}_{3}$ ) of $\mathbf{1 t}$

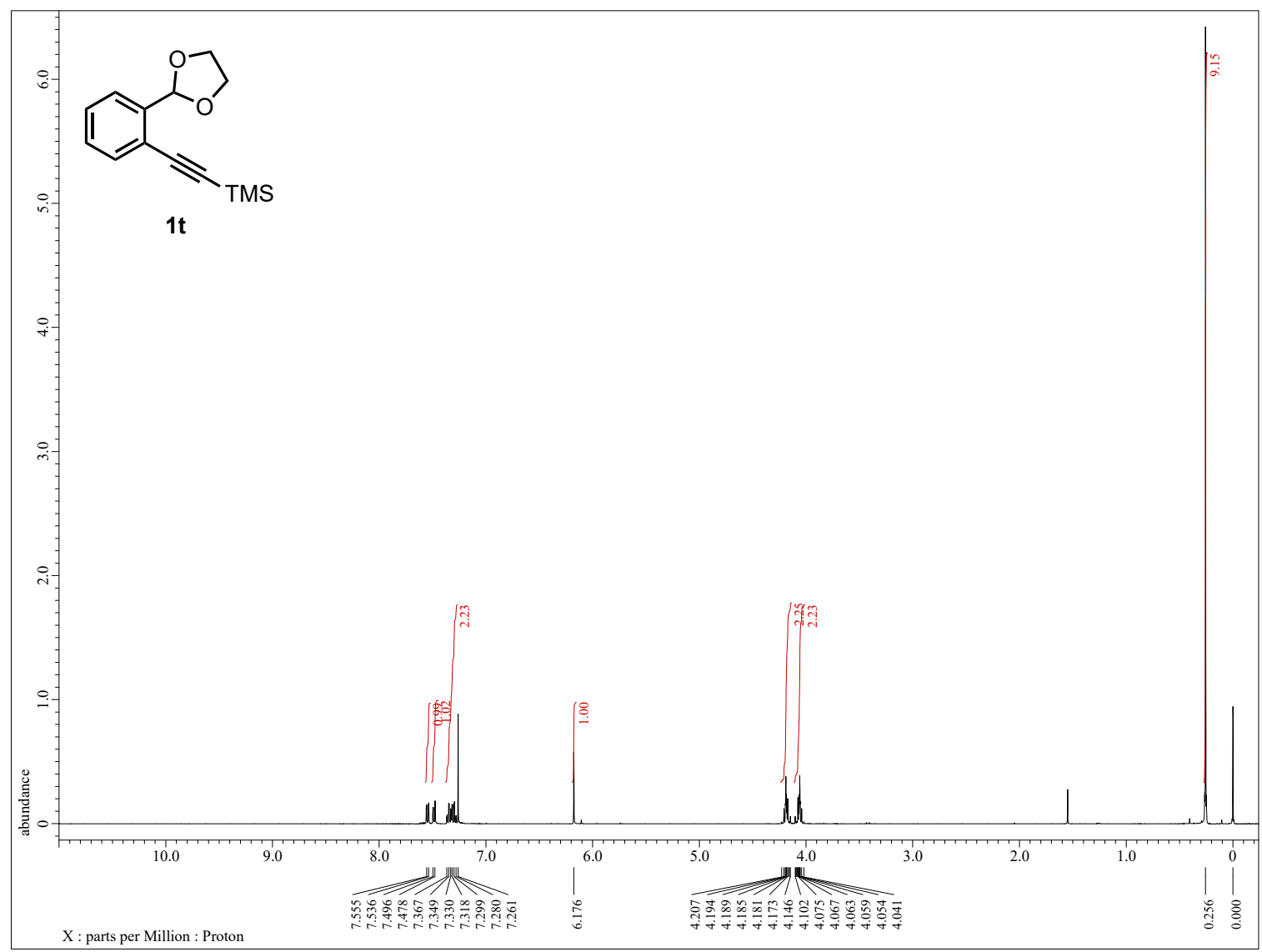


${ }^{1} \mathrm{H}$ NMR (400 MHz, $\mathrm{CDCl}_{3}$ ) of $\mathbf{1 u}$

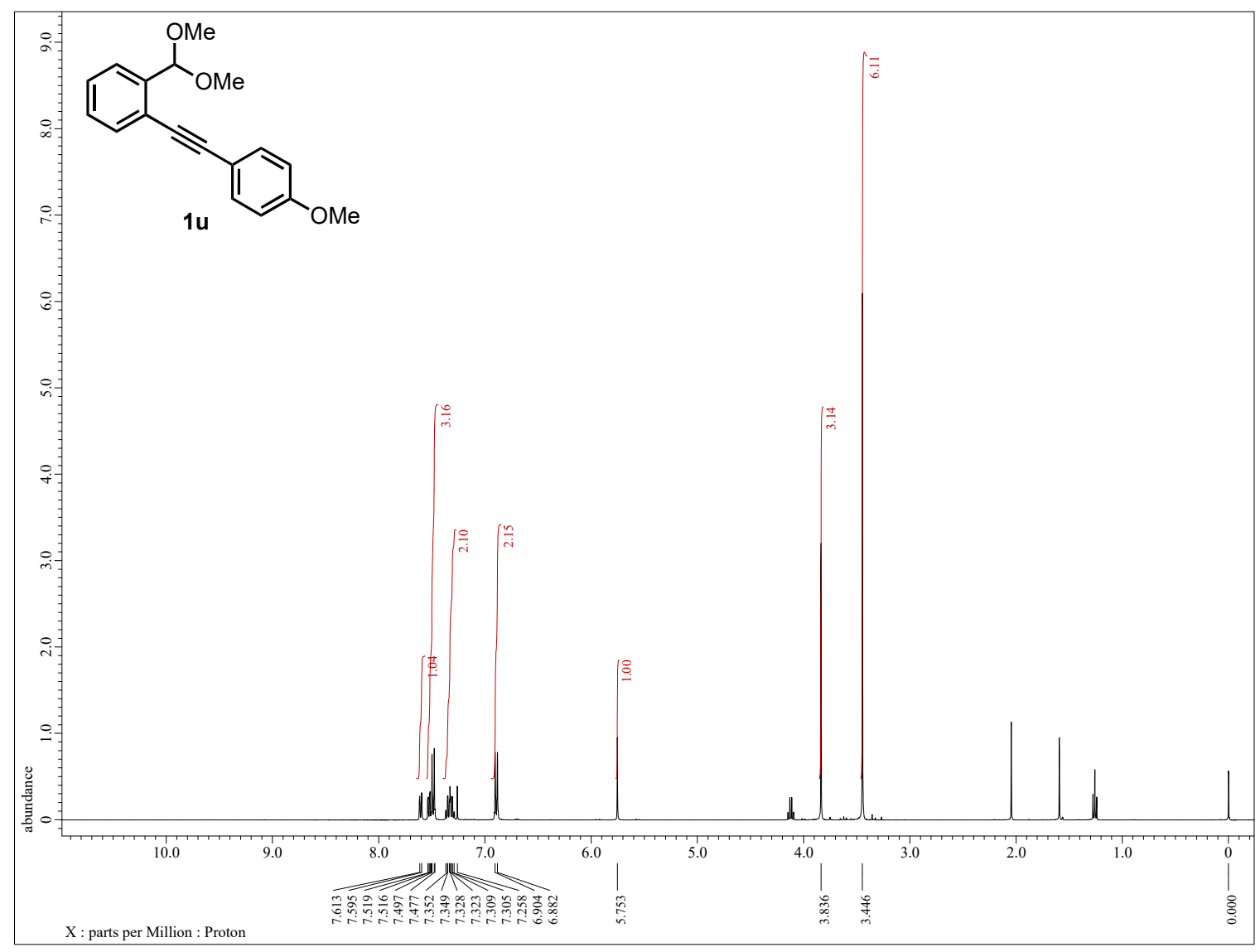

${ }^{1} \mathrm{H}$ NMR (400 MHz, $\mathrm{CDCl}_{3}$ ) of $\mathbf{S 5}$

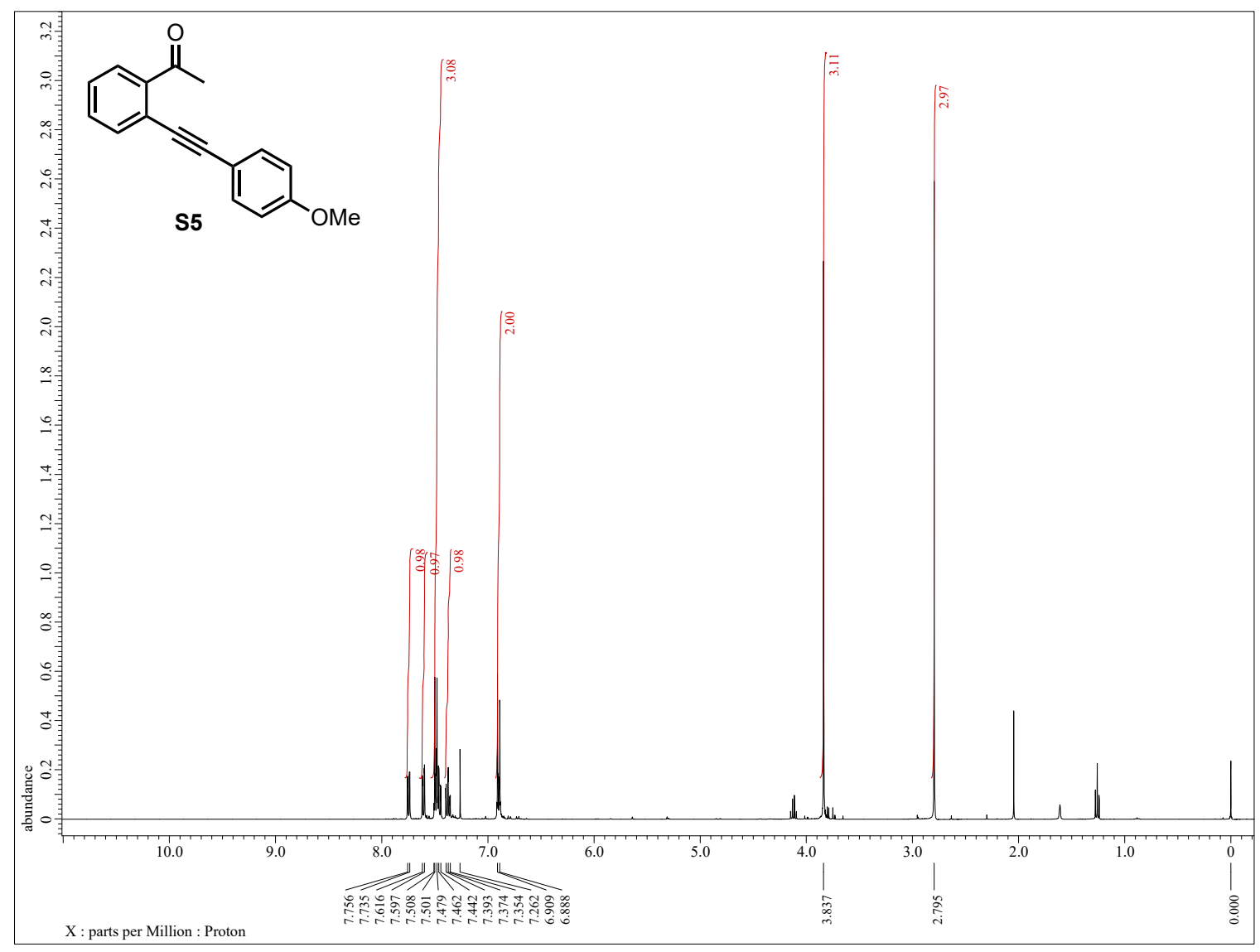

S64 
${ }^{1} \mathrm{H}$ NMR $\left(400 \mathrm{MHz}, \mathrm{CDCl}_{3}\right)$ of 6

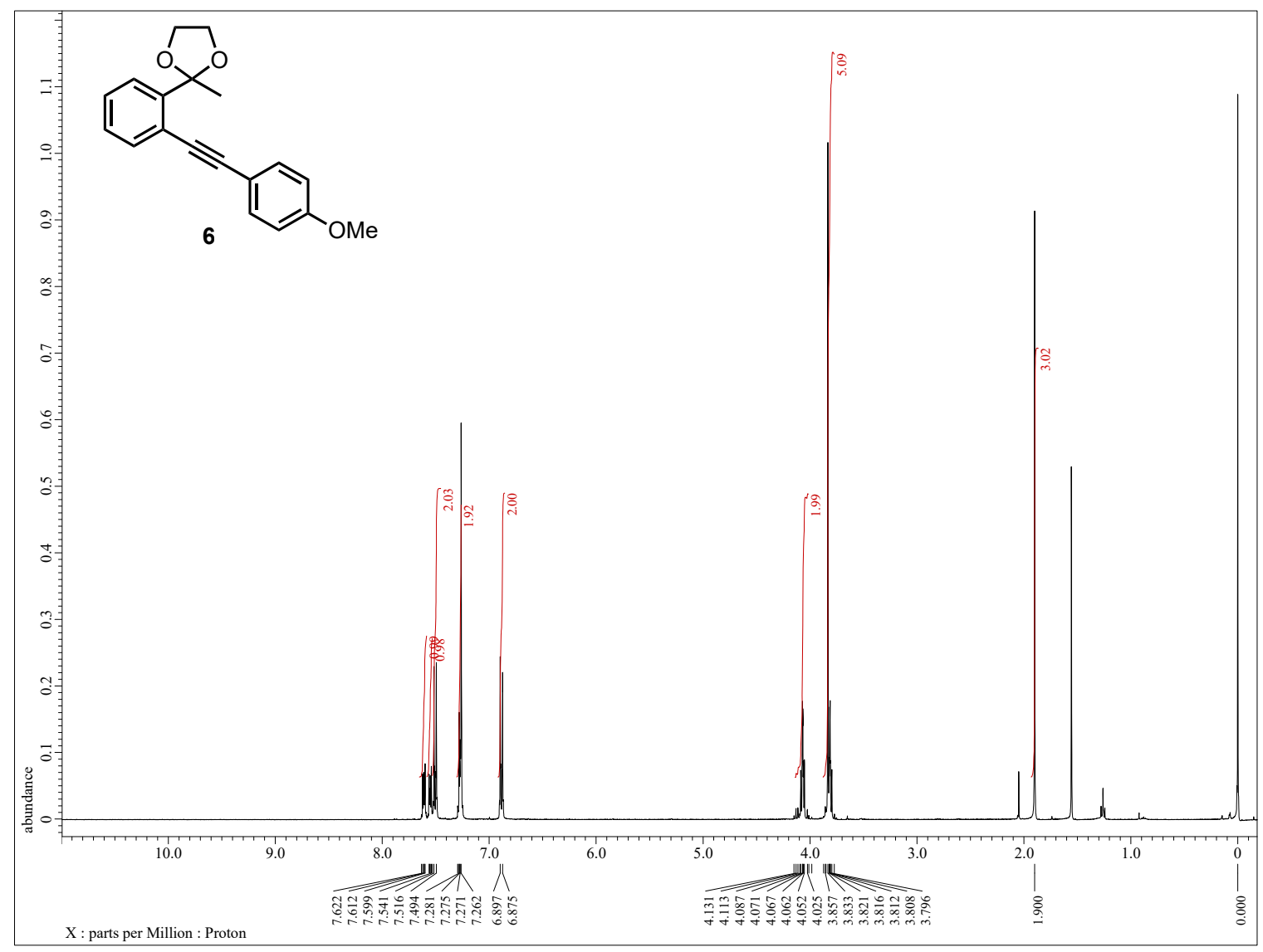

${ }^{13} \mathrm{C}$ NMR $\left(100 \mathrm{MHz}, \mathrm{CDCl}_{3}\right)$ of $\mathbf{6}$

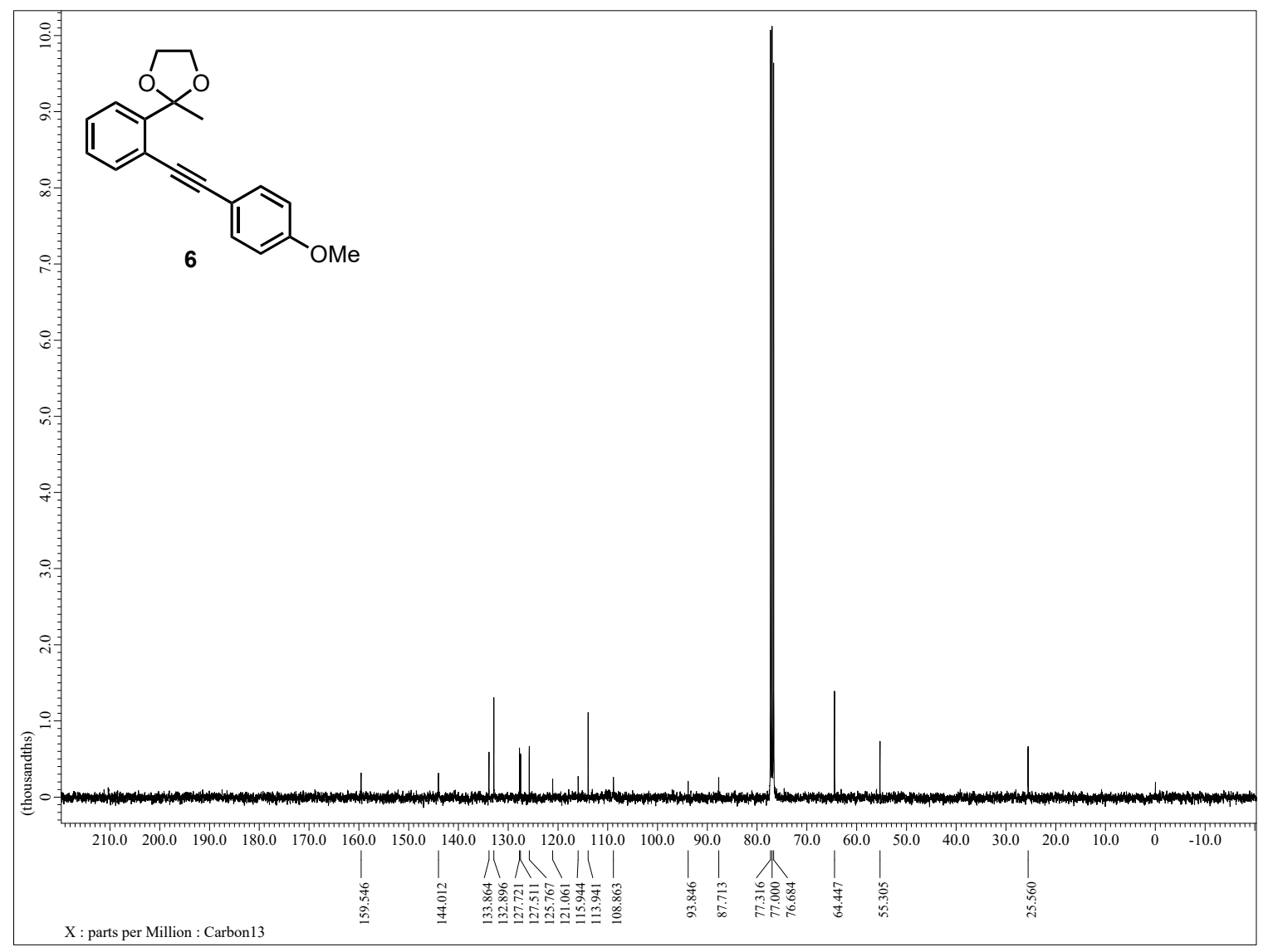

S65 
${ }^{1} \mathrm{H}$ NMR $\quad\left(400 \mathrm{MHz}, \mathrm{CDCl}_{3}\right)$ of 7

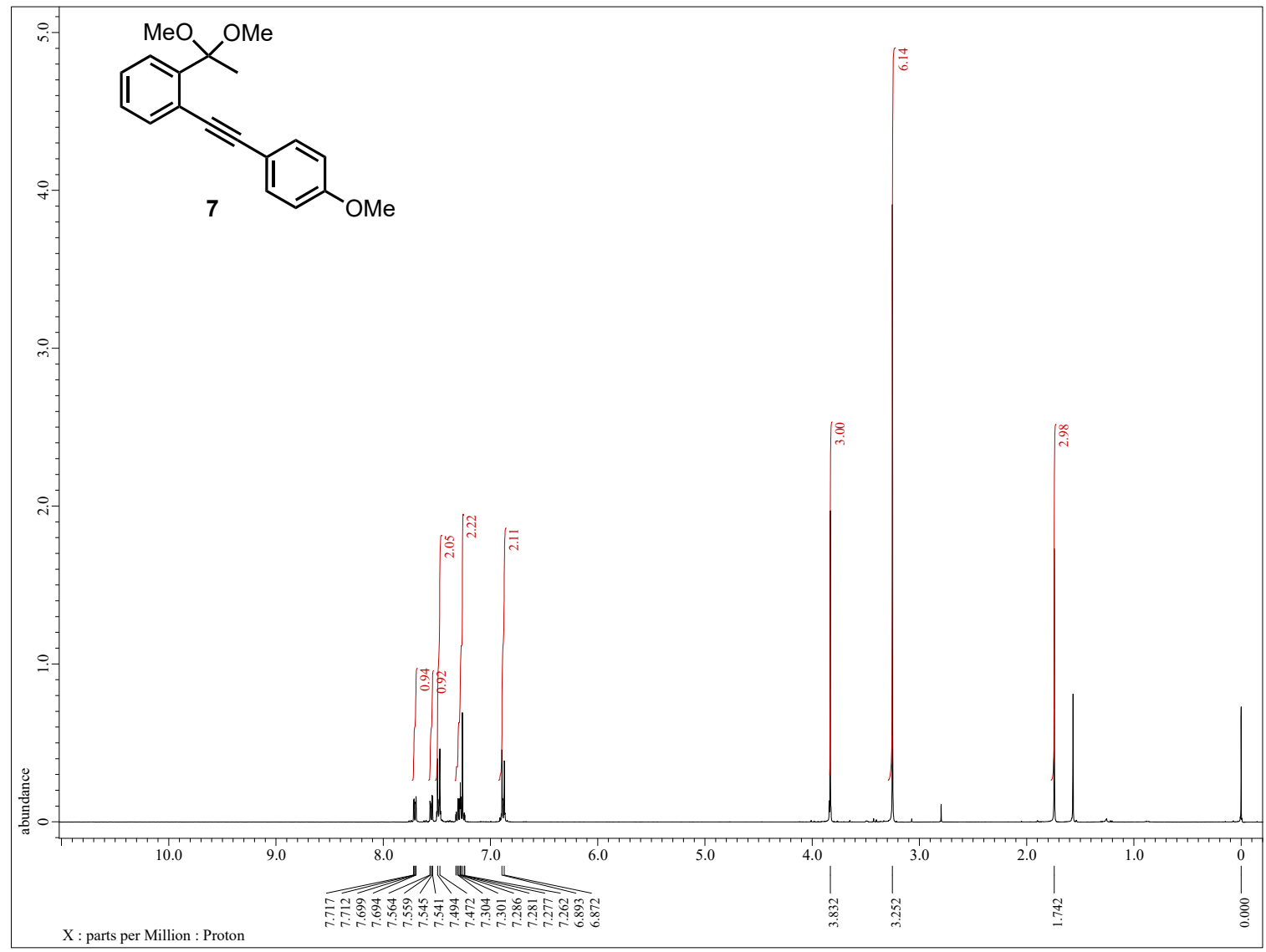

${ }^{13} \mathrm{C}$ NMR $\left(100 \mathrm{MHz}, \mathrm{CDCl}_{3}\right)$ of 7

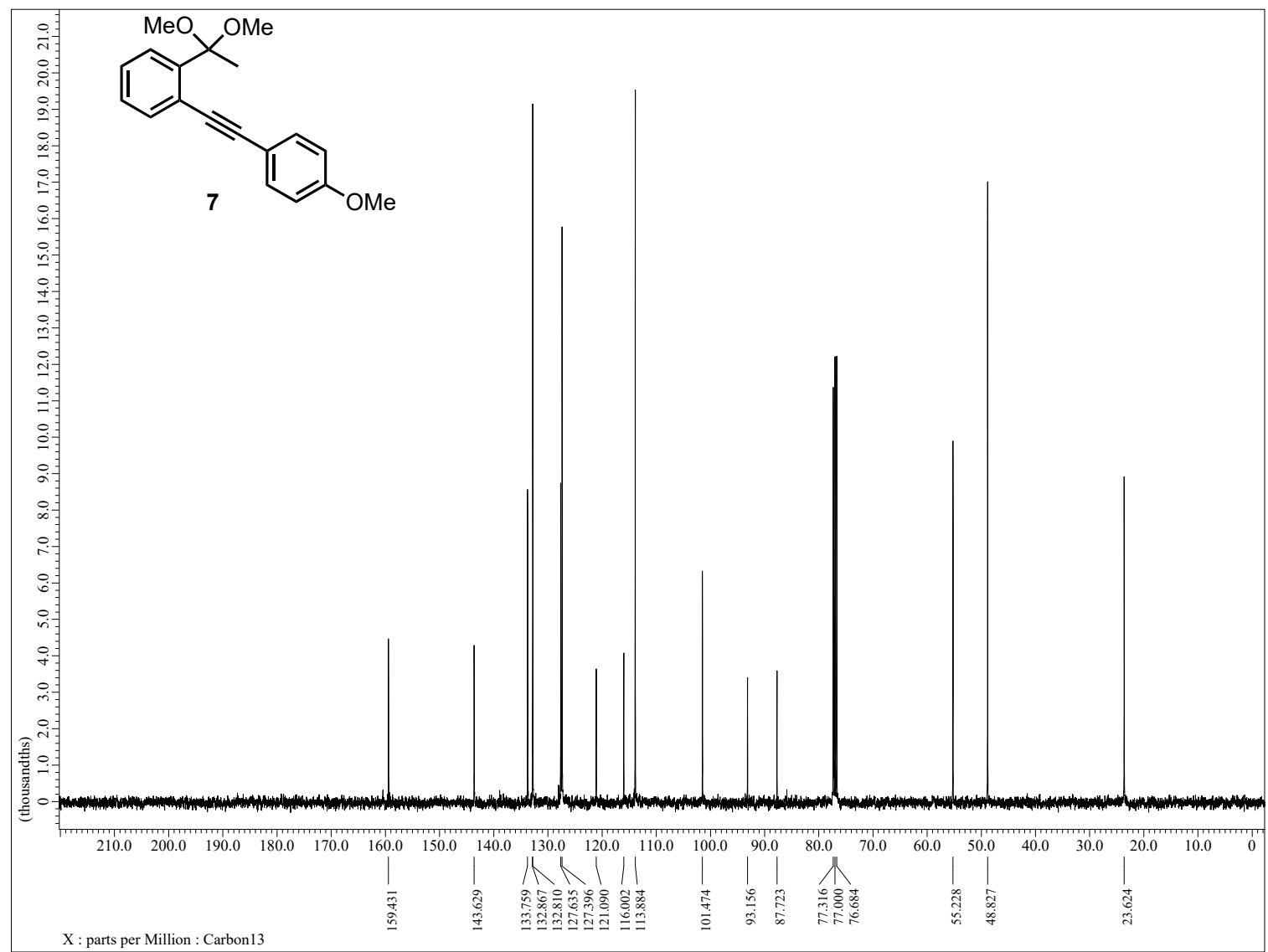


${ }^{1} \mathrm{H}$ NMR (400 MHz, $\mathrm{CDCl}_{3}$ ) of $\mathbf{S 1 j - 1 - \mathbf { d } _ { 2 }}$

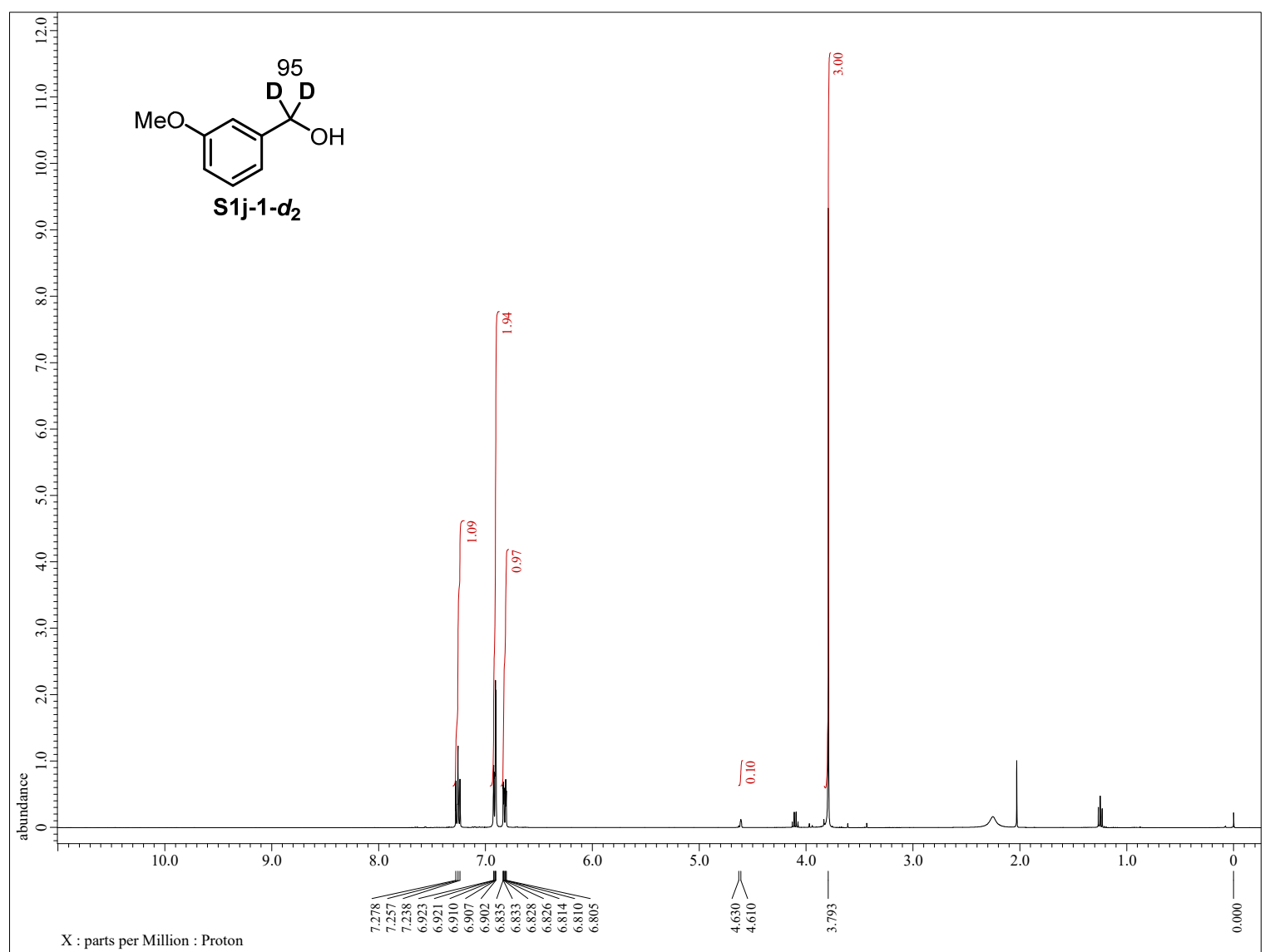

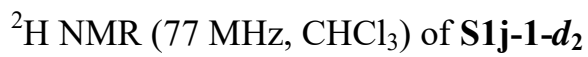

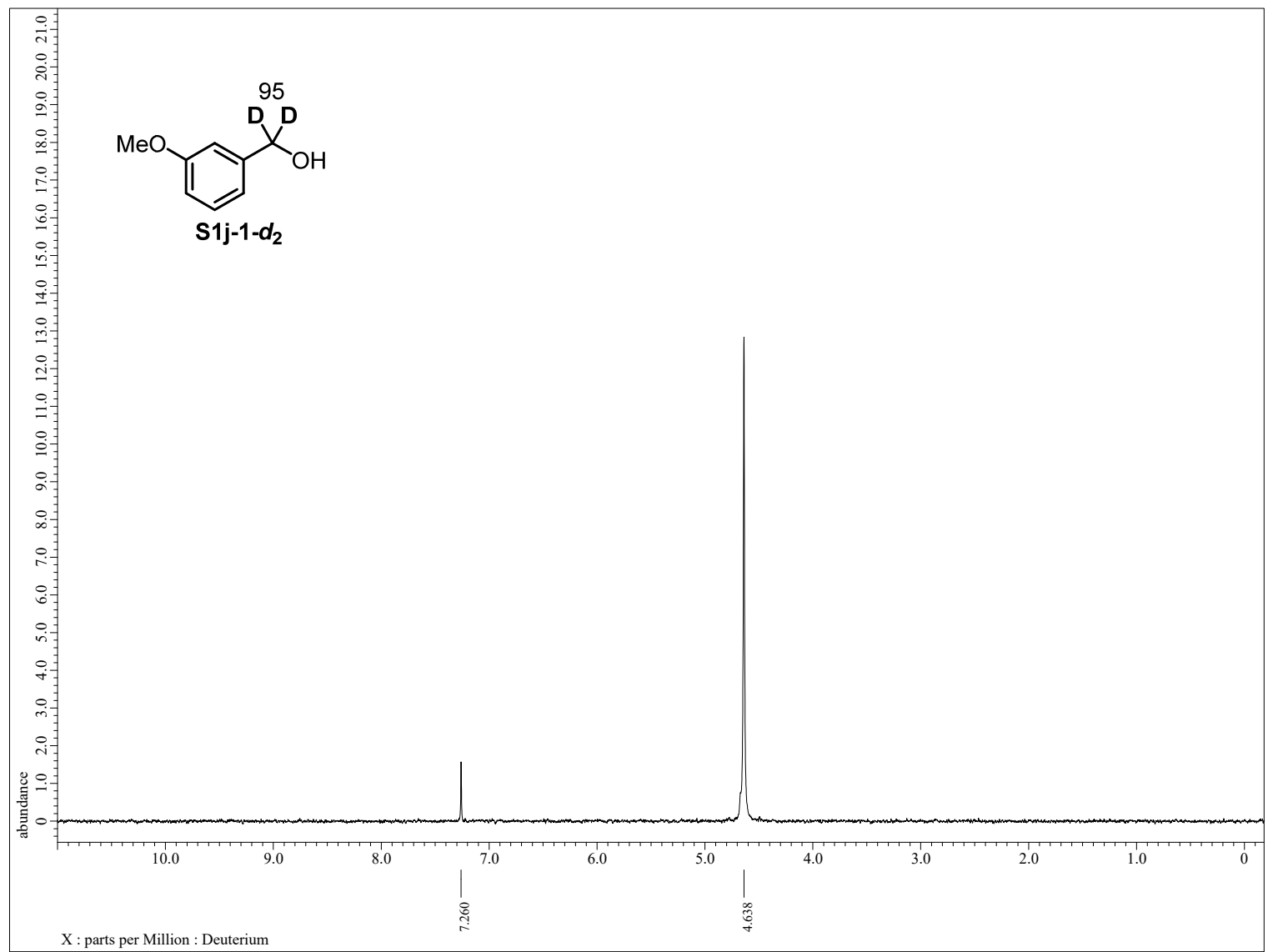


${ }^{1} \mathrm{H}$ NMR $\quad\left(400 \mathrm{MHz}, \mathrm{CDCl}_{3}\right)$ of $\mathbf{S 1 j - 2 - \boldsymbol { d } _ { \mathbf { 1 } }}$

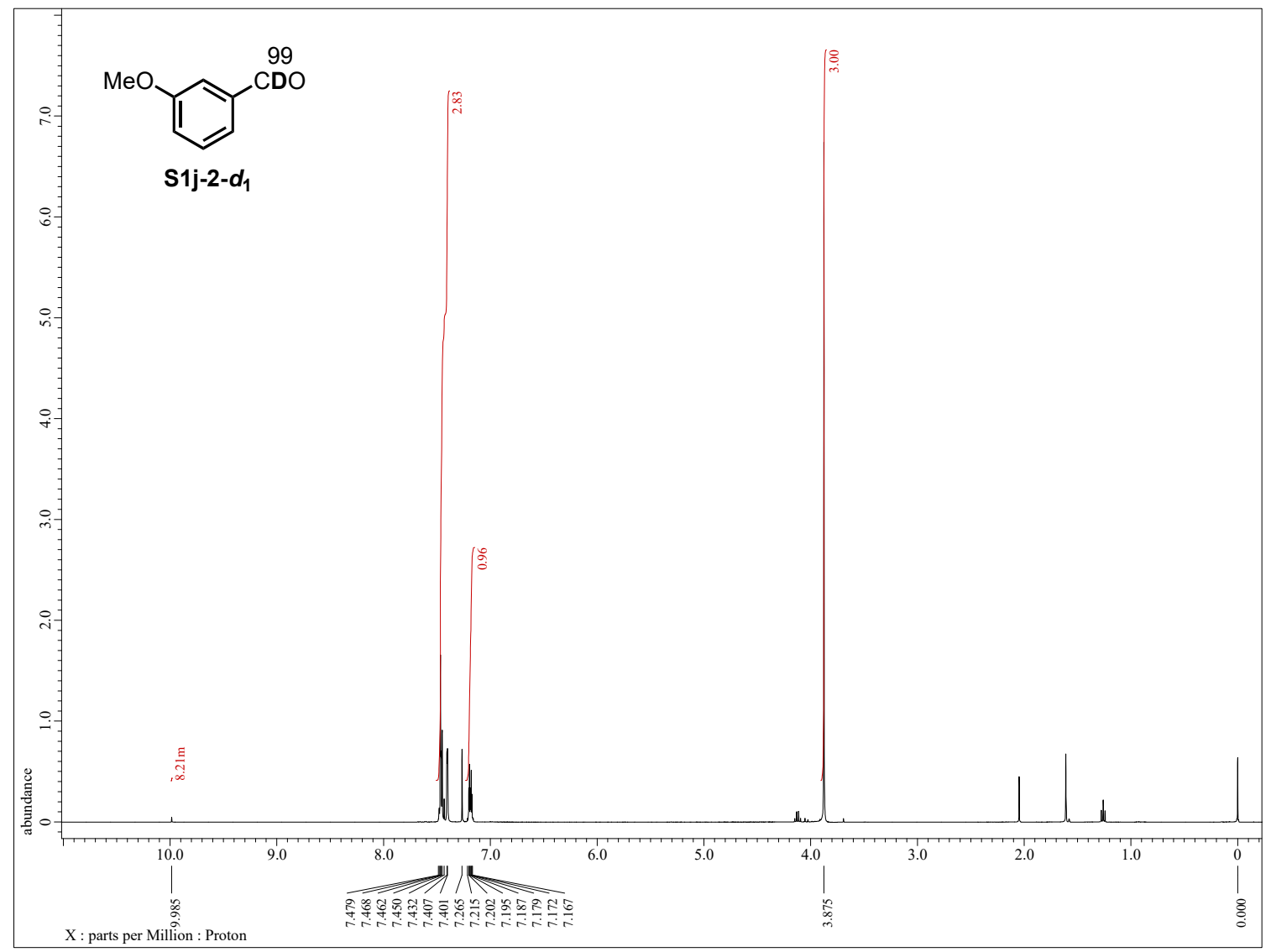

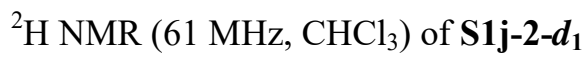

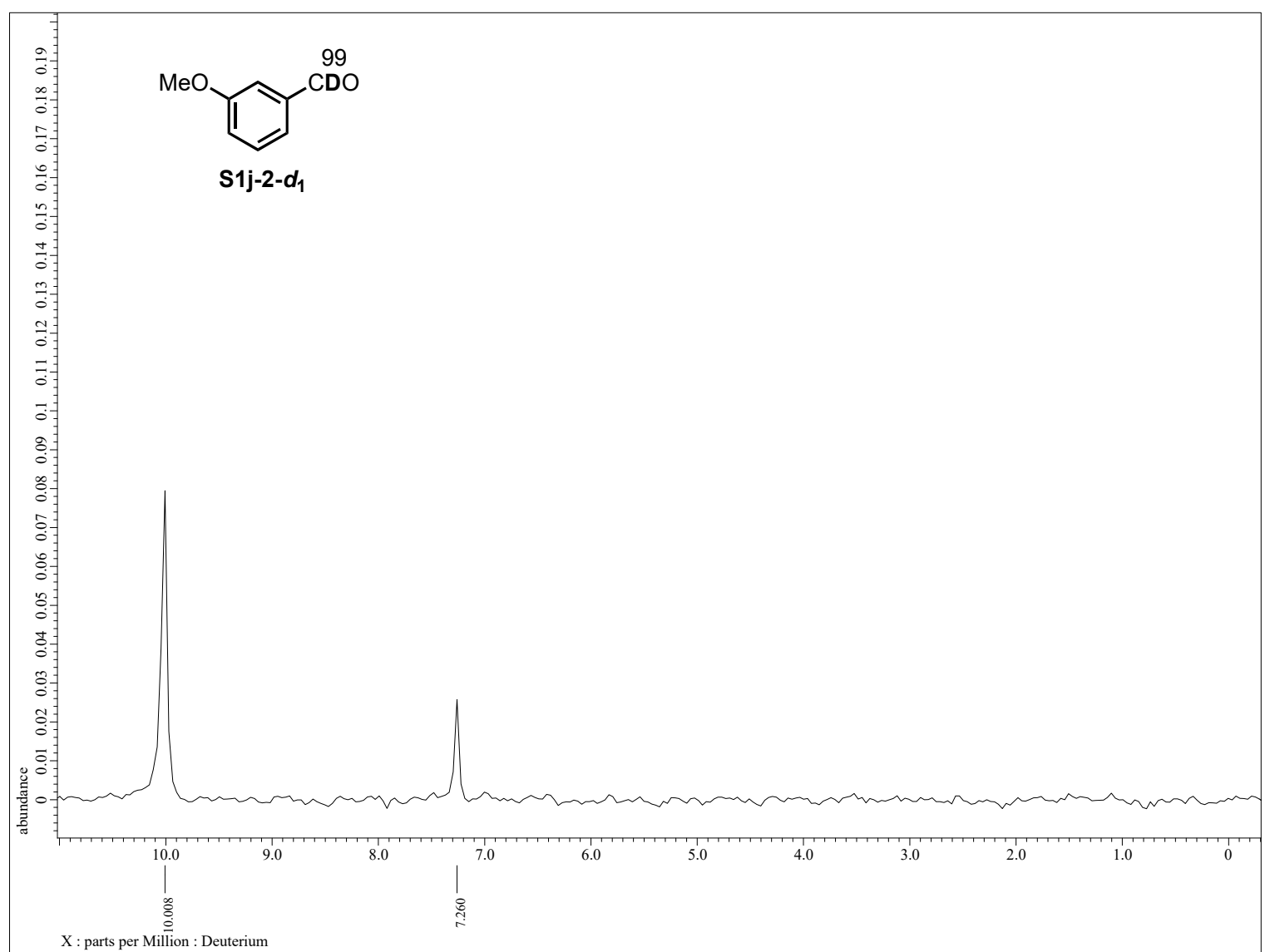


${ }^{1} \mathrm{H}$ NMR $\quad\left(400 \mathrm{MHz}, \mathrm{CDCl}_{3}\right)$ of $\mathbf{S 1 j - 3 - d _ { \mathbf { 1 } }}$

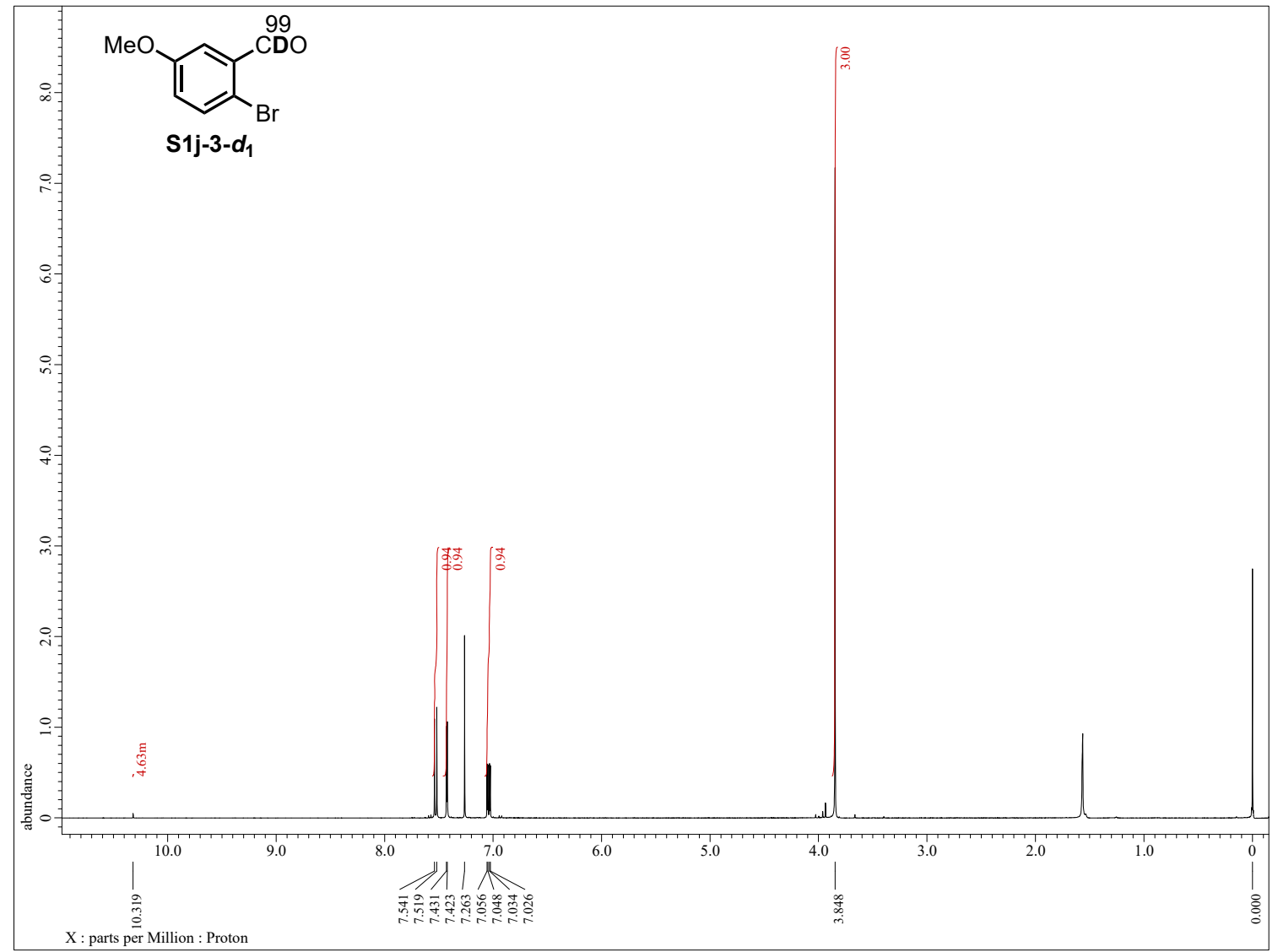

${ }^{2} \mathrm{H}$ NMR (61 MHz, $\mathrm{CHCl}_{3}$ ) of $\mathbf{S} \mathbf{1 j - 3}-\mathbf{d}_{\mathbf{1}}$

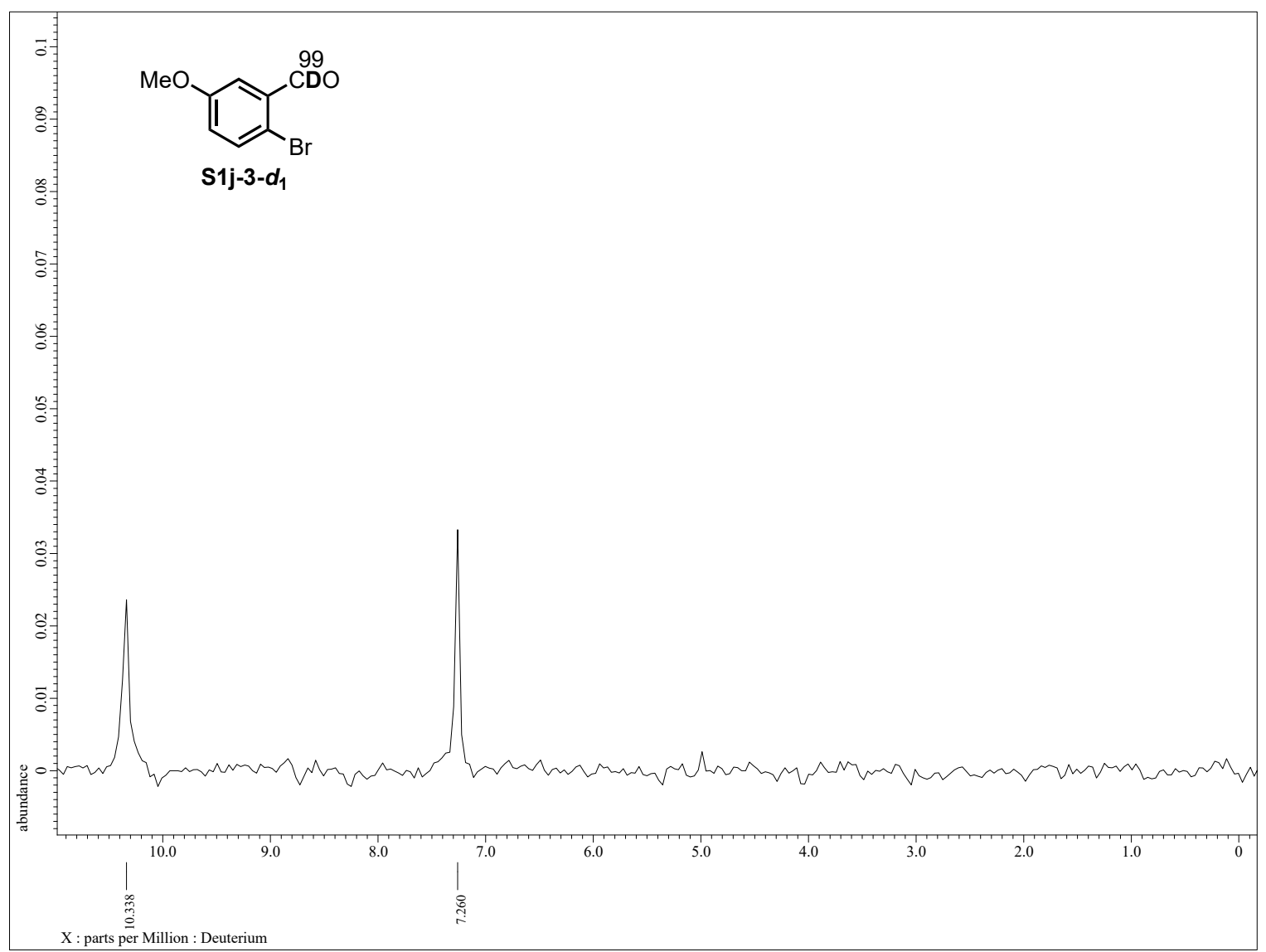


${ }^{1} \mathrm{H}$ NMR $\quad\left(400 \mathrm{MHz}, \mathrm{CDCl}_{3}\right)$ of $\mathbf{S} \mathbf{1} \mathbf{j}-\boldsymbol{d}_{\mathbf{1}}$

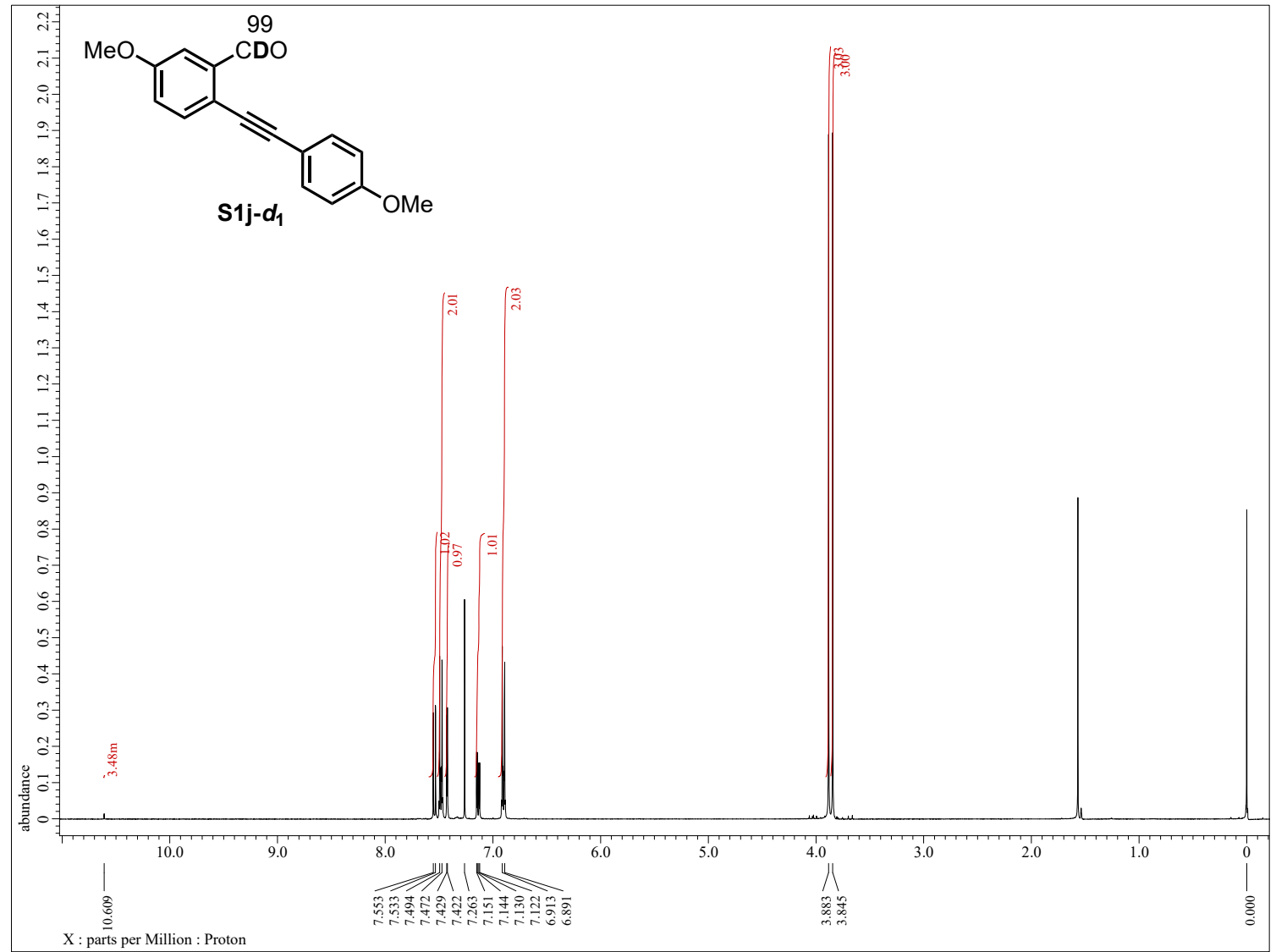

${ }^{2} \mathrm{H}$ NMR (77 MHz, $\mathrm{CHCl}_{3}$ ) of $\mathbf{S} \mathbf{1} \mathbf{j}-\boldsymbol{d}_{\mathbf{1}}$

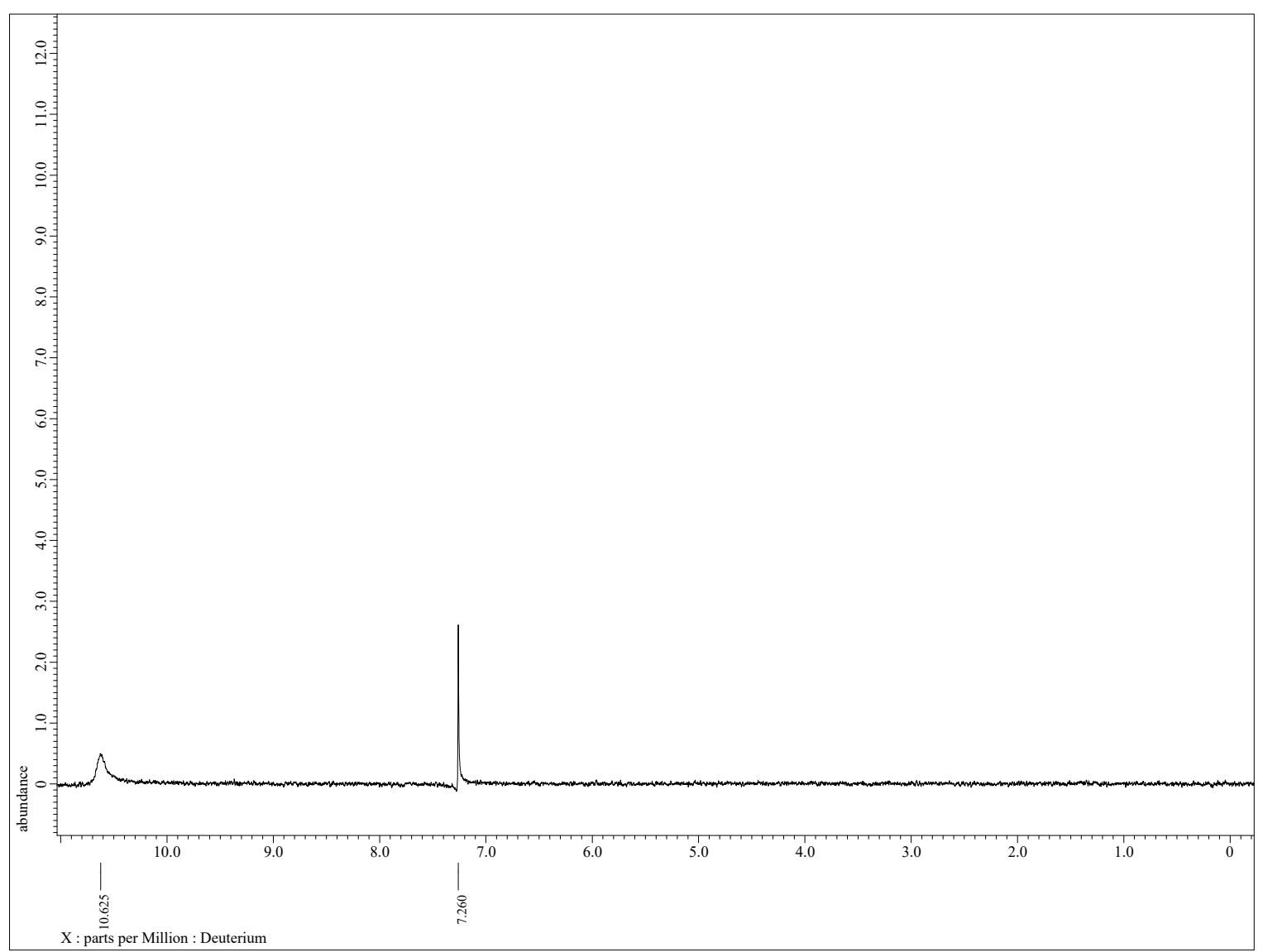


${ }^{1} \mathrm{H}$ NMR $\quad\left(400 \mathrm{MHz}, \mathrm{CDCl}_{3}\right)$ of $\mathbf{1} \mathbf{j}-\mathbf{d}_{\mathbf{1}}$

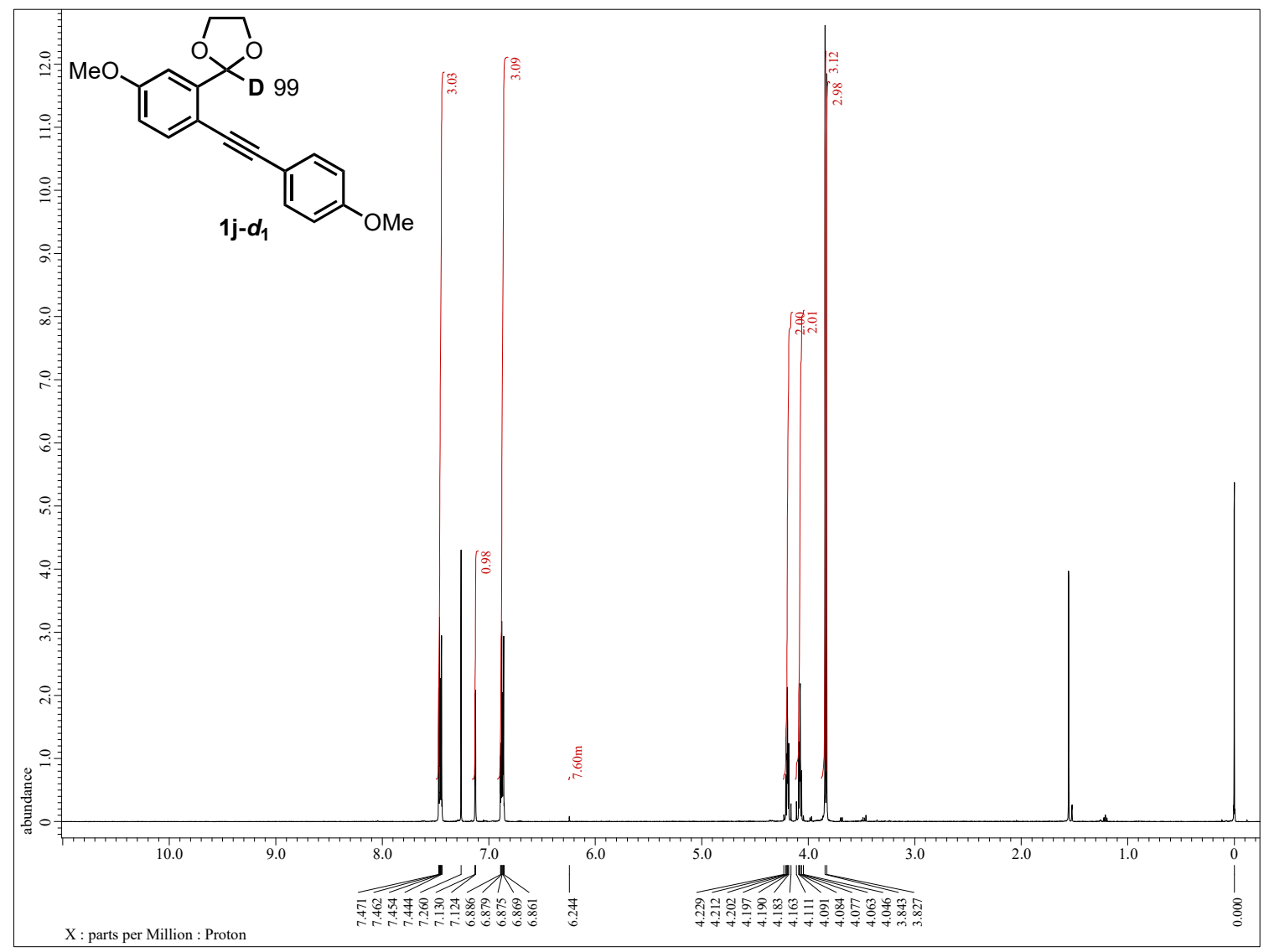

${ }^{2} \mathrm{H}$ NMR (61 MHz, $\left.\mathrm{CHCl}_{3}\right)$ of $\mathbf{1} \mathbf{j}-\boldsymbol{d}_{\mathbf{1}}$

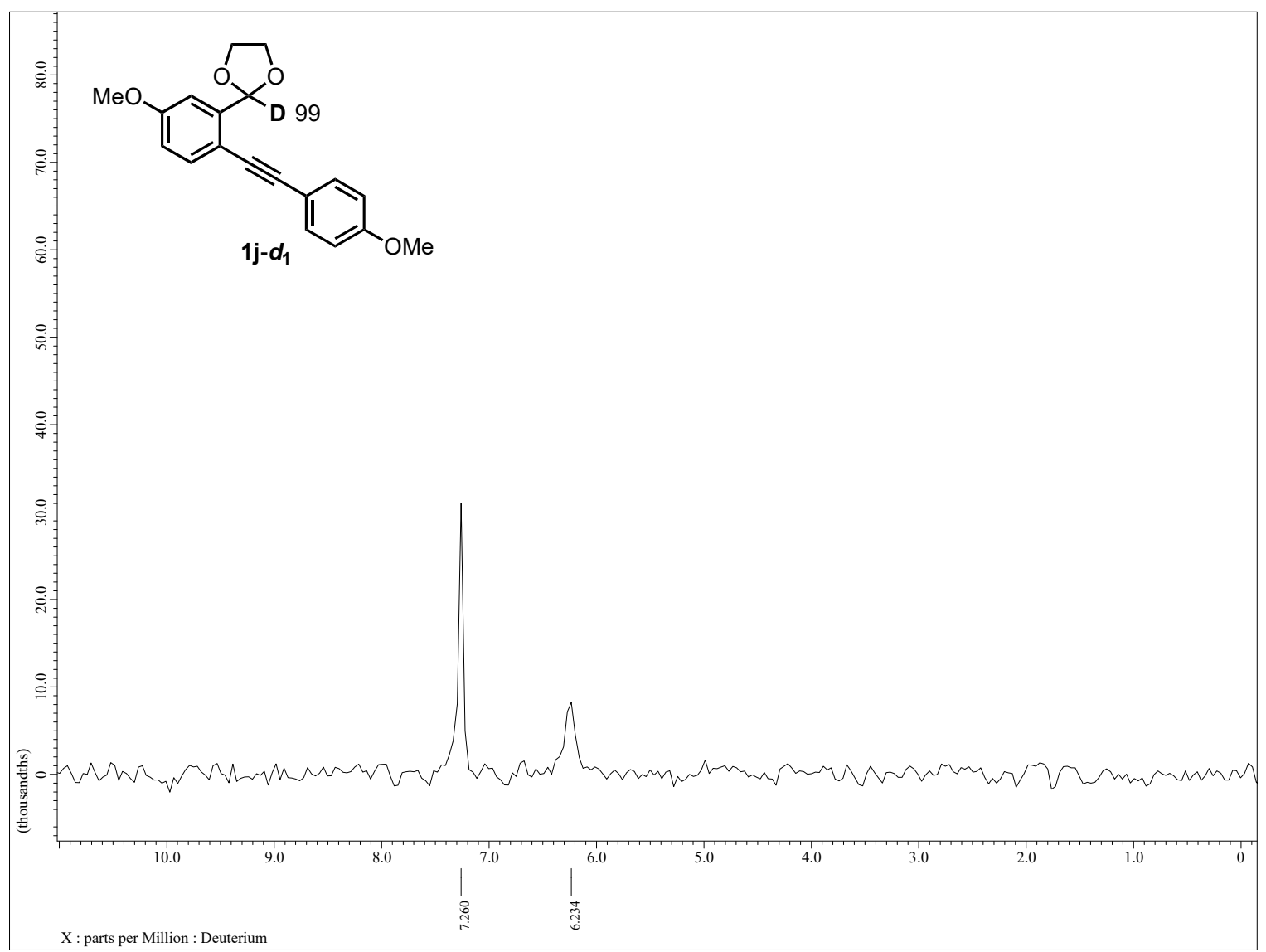


${ }^{1} \mathrm{H}$ NMR $\quad\left(400 \mathrm{MHz}, \mathrm{CDCl}_{3}\right)$ of 8

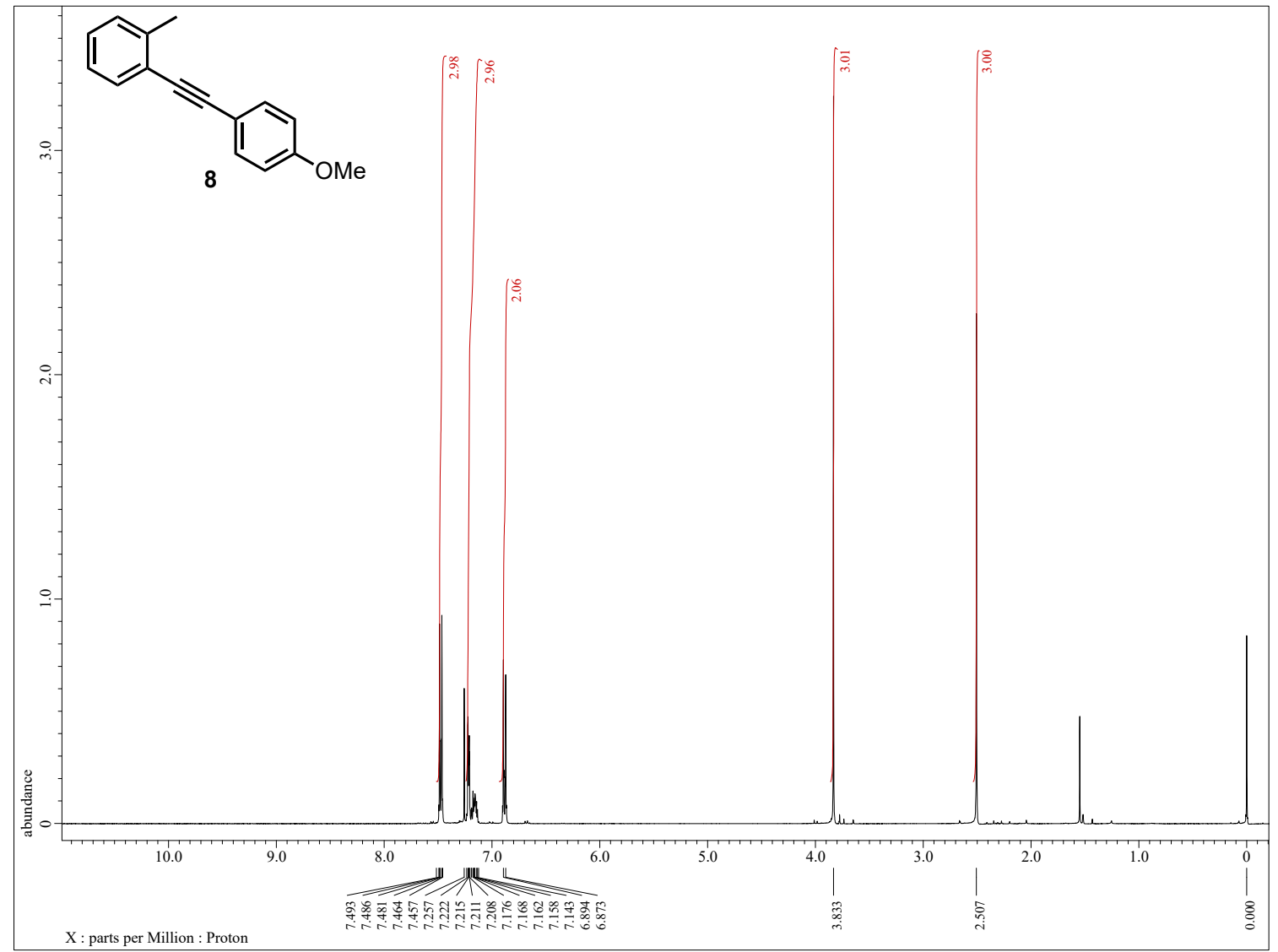

${ }^{1} \mathrm{H}$ NMR $\quad\left(400 \mathrm{MHz}, \mathrm{CDCl}_{3}\right)$ of $\mathbf{S 1 0 - 1}$

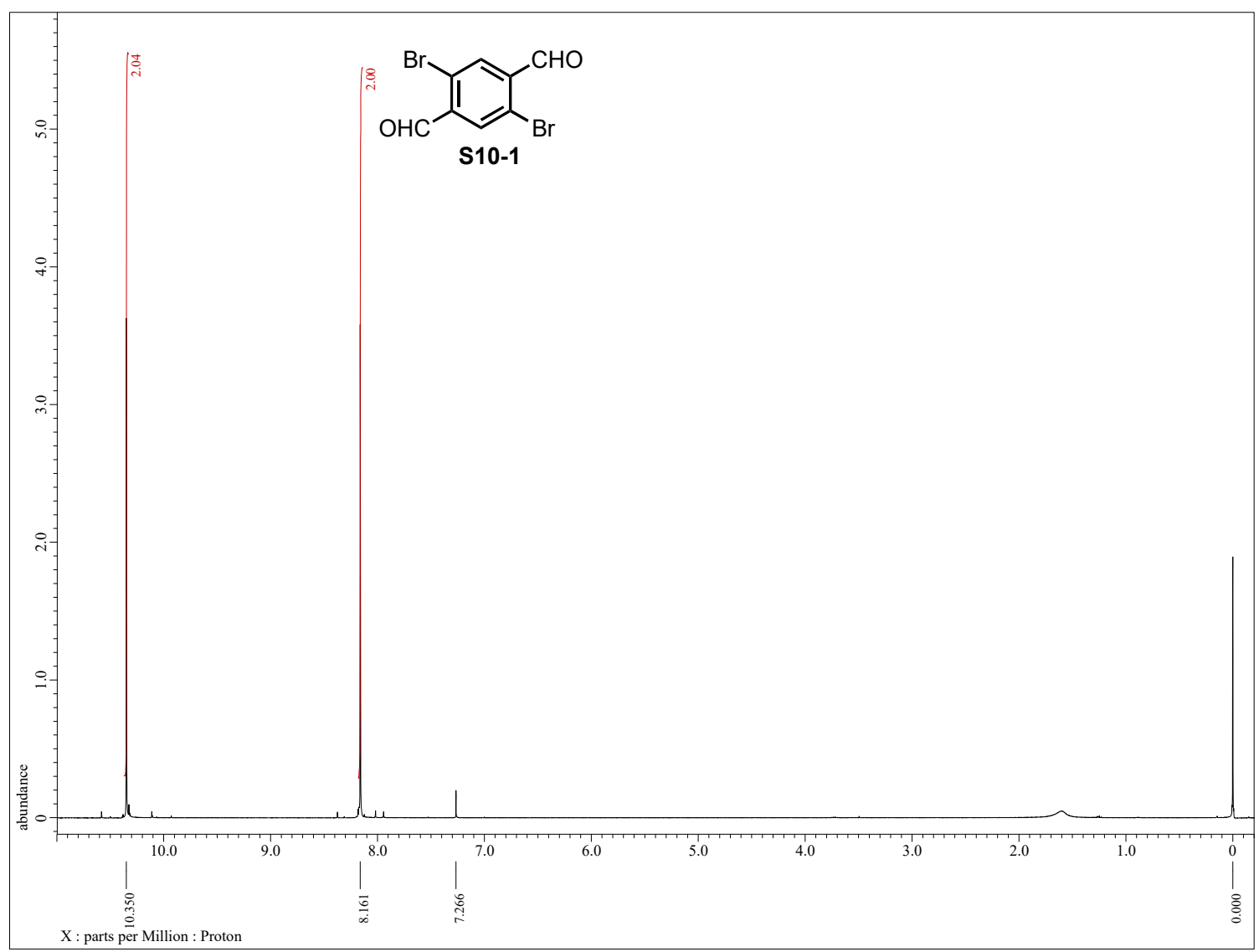


${ }^{1} \mathrm{H}$ NMR (400 MHz, $\mathrm{CDCl}_{3}$ ) of $\mathbf{S 1 0 - 2}$

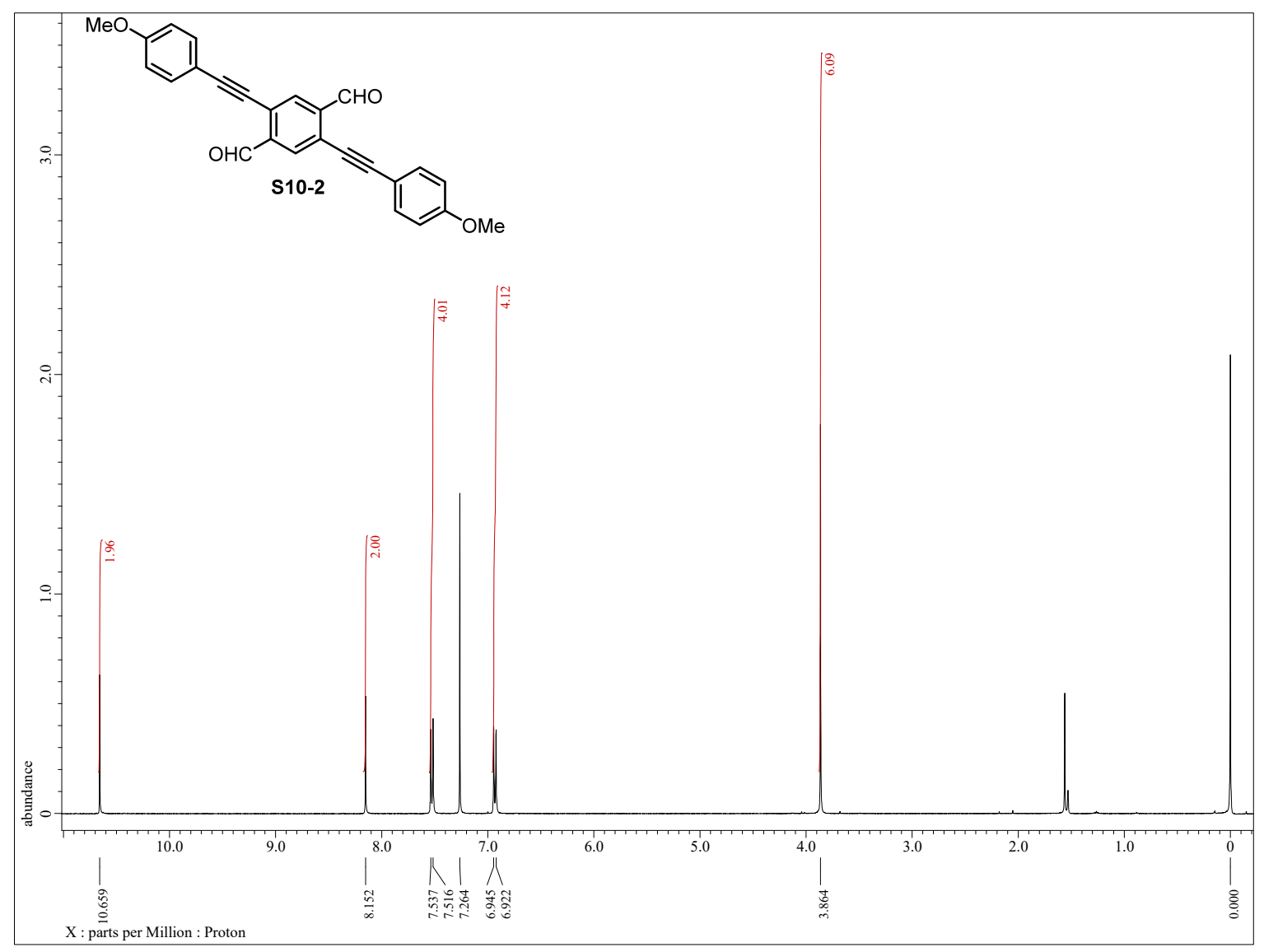

${ }^{13} \mathrm{C}$ NMR $\left(100 \mathrm{MHz}, \mathrm{CDCl}_{3}\right)$ of $\mathbf{S 1 0 - 2}$

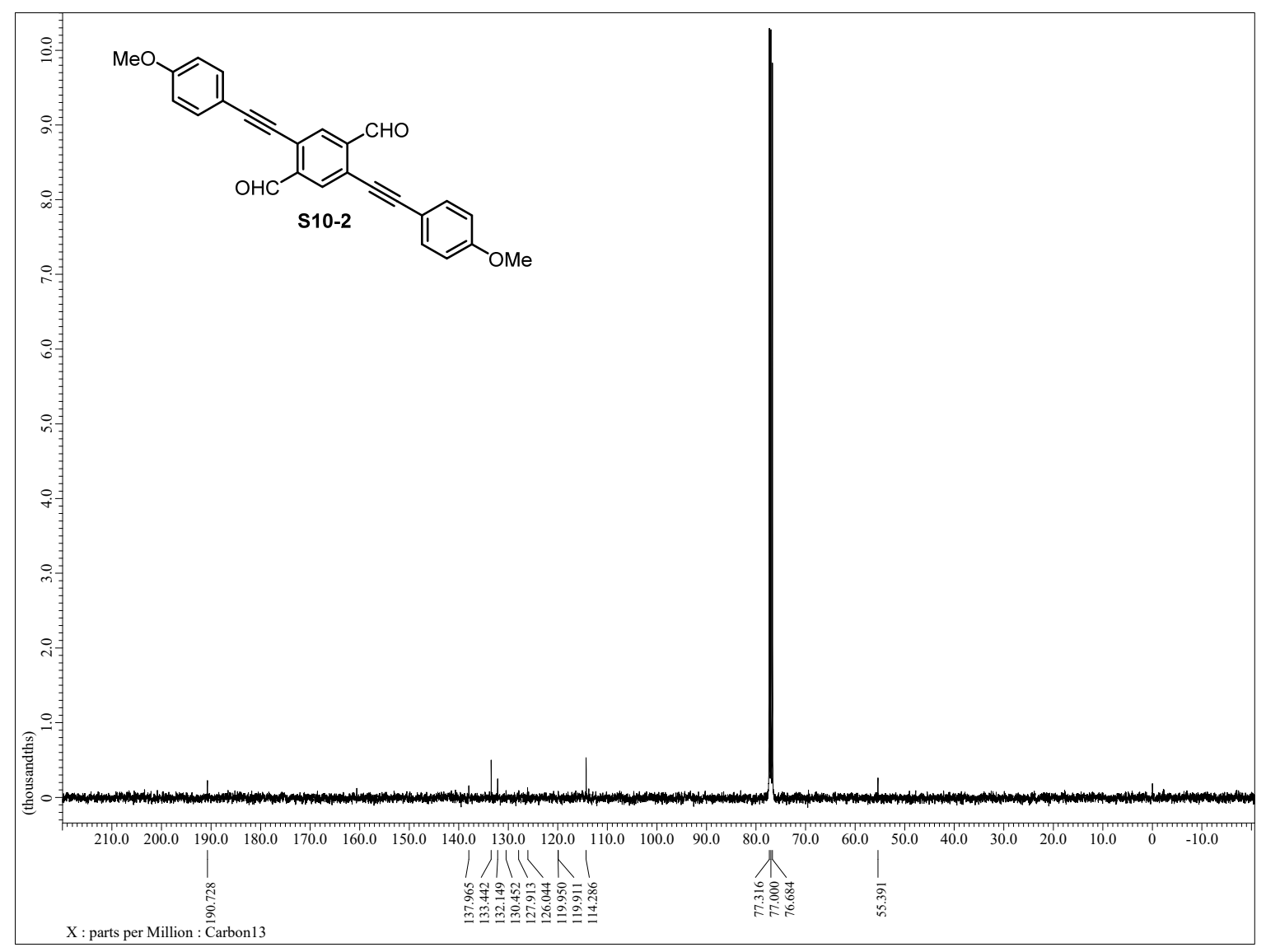

S73 
${ }^{1} \mathrm{H}$ NMR $\quad\left(400 \mathrm{MHz}, \mathrm{CDCl}_{3}\right)$ of $\mathbf{1 0}$

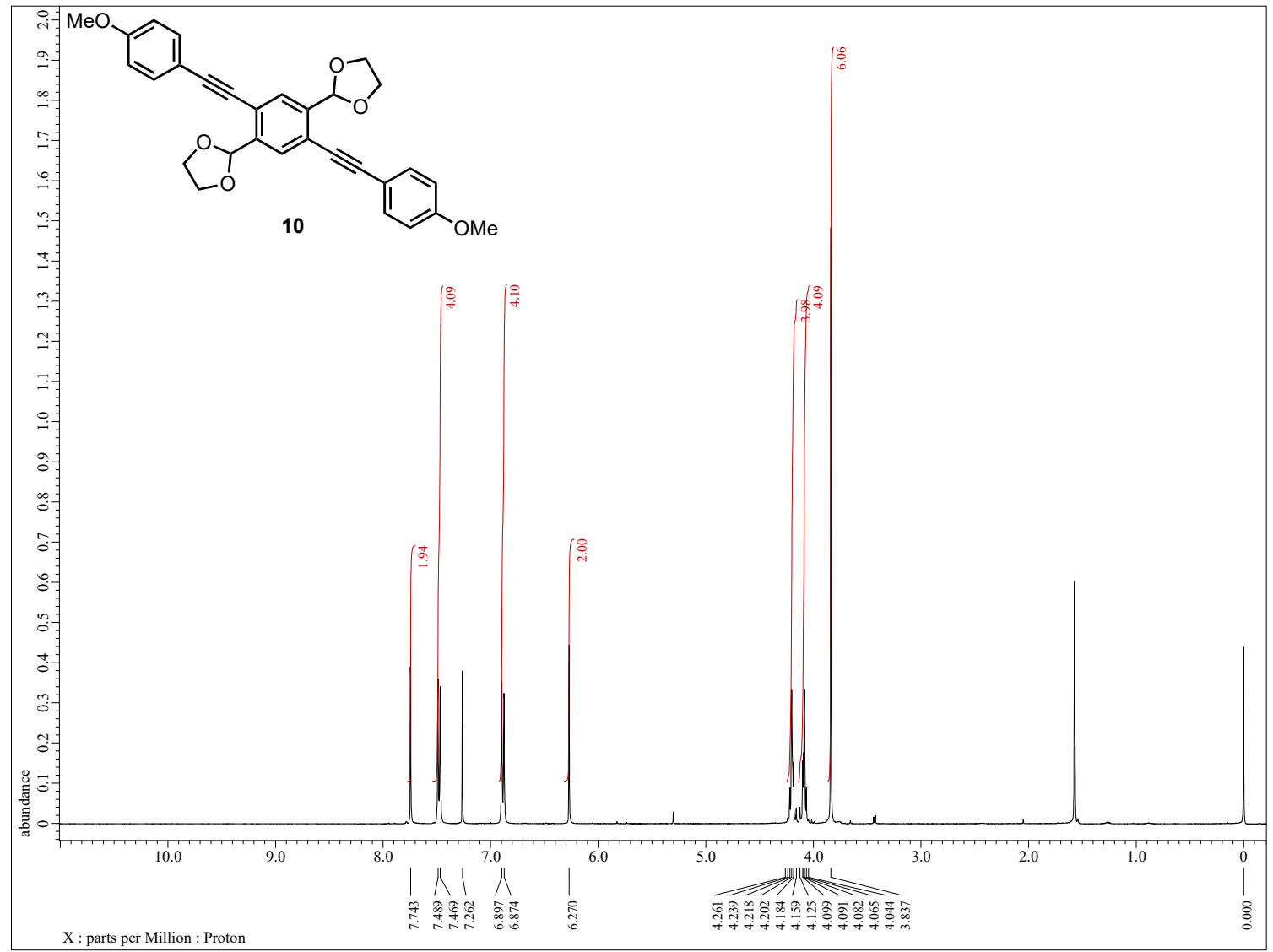

${ }^{13} \mathrm{C}$ NMR $\quad\left(100 \mathrm{MHz}, \mathrm{CDCl}_{3}\right)$ of $\mathbf{1 0}$

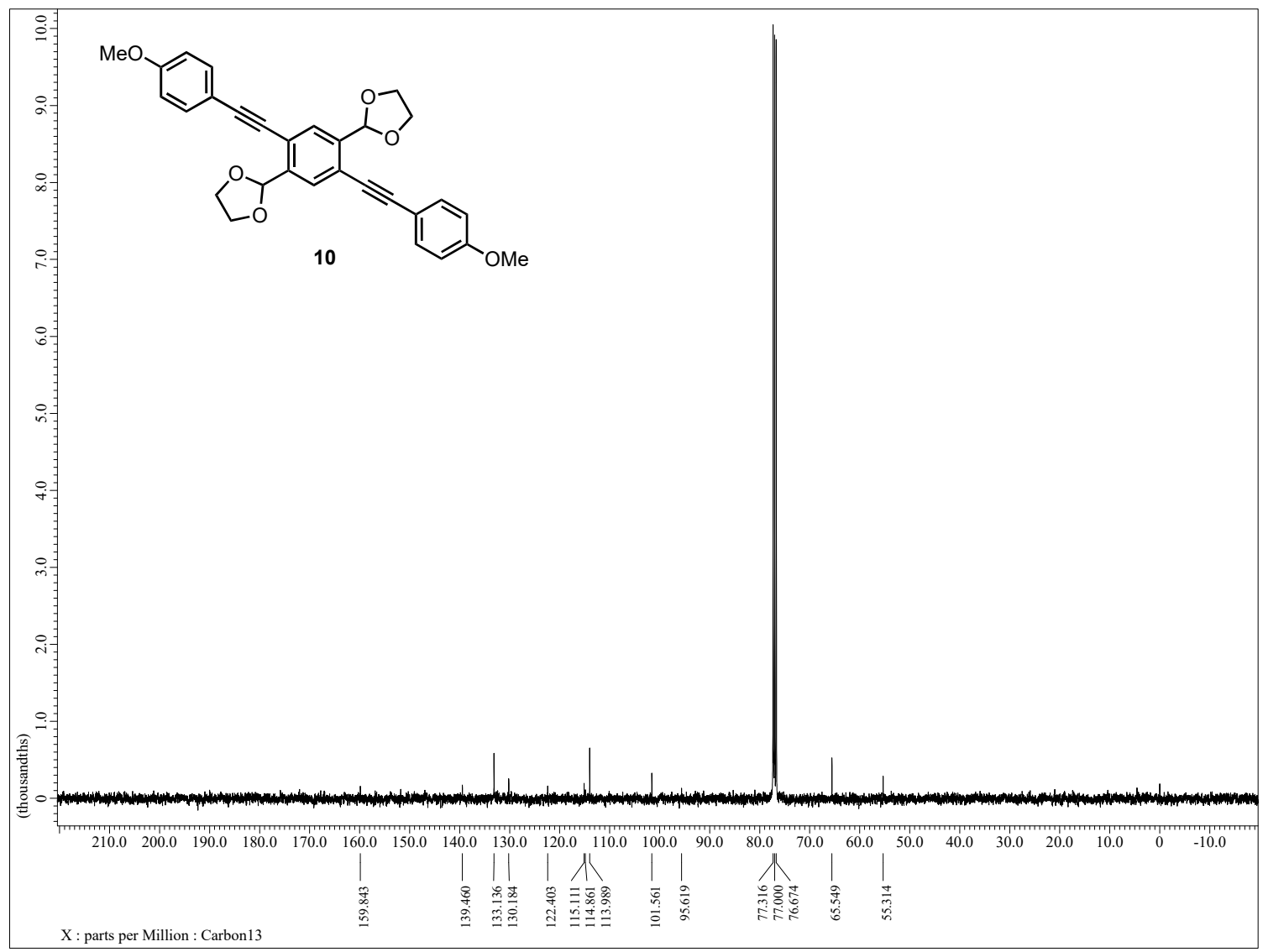


${ }^{1} \mathrm{H}$ NMR (500 MHz, $\mathrm{CDCl}_{3}$ ) of $\mathbf{1 2}$

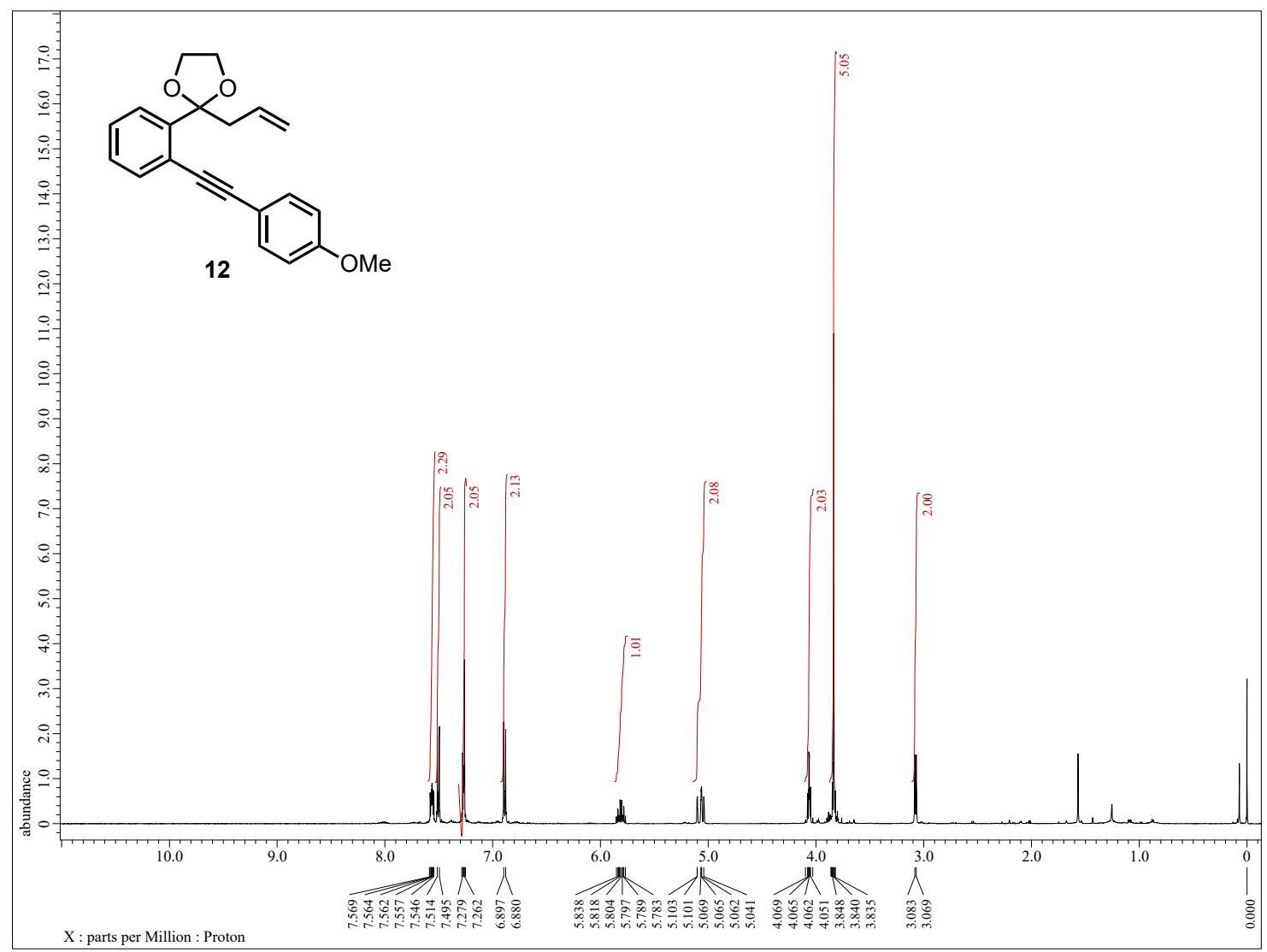

${ }^{13} \mathrm{C}$ NMR $\left(125 \mathrm{MHz}, \mathrm{CDCl}_{3}\right)$ of $\mathbf{1 2}$

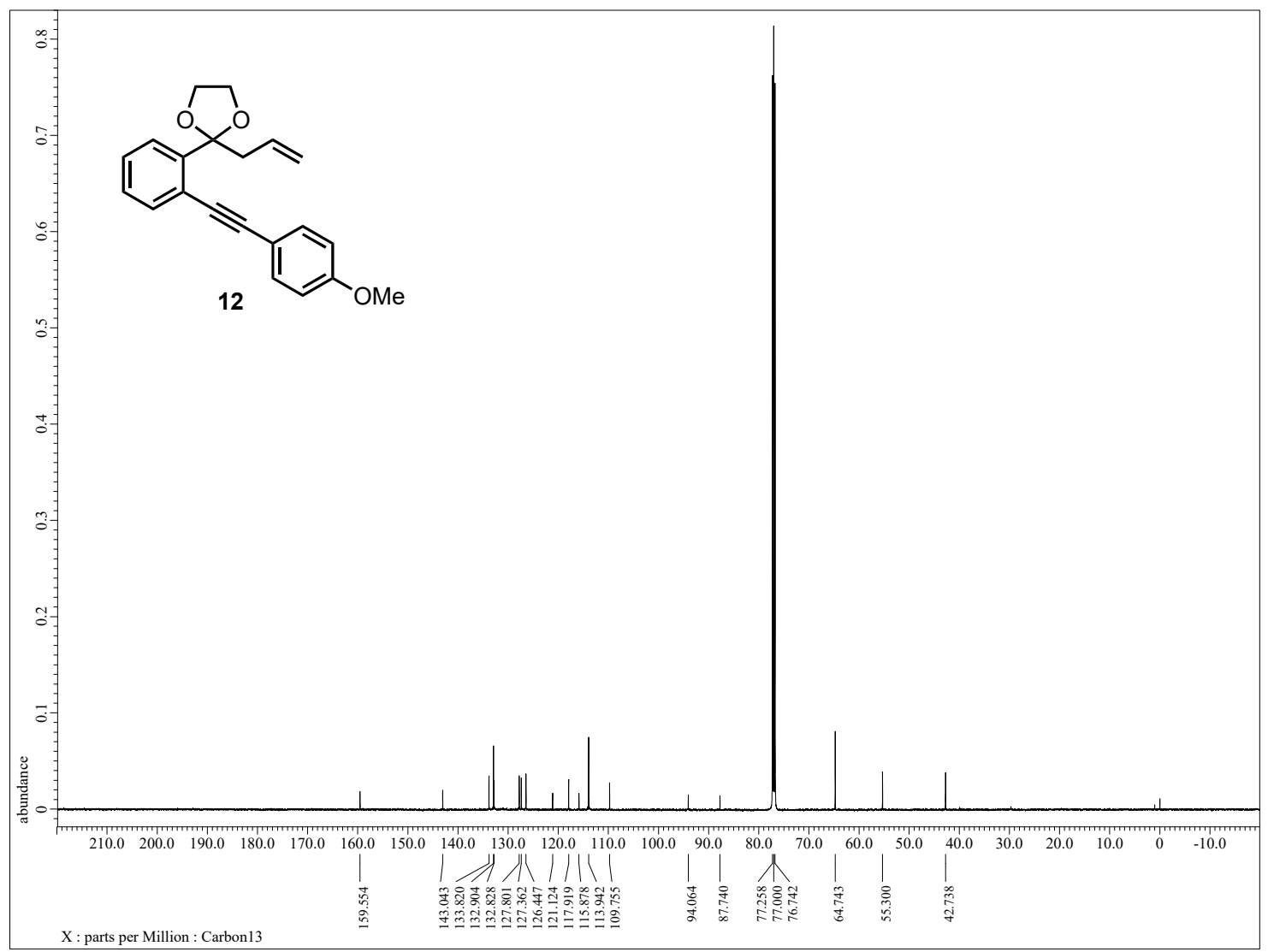


${ }^{1} \mathrm{H}$ NMR $\quad\left(400 \mathrm{MHz}, \mathrm{CDCl}_{3}\right)$ of $\mathbf{2 a}$

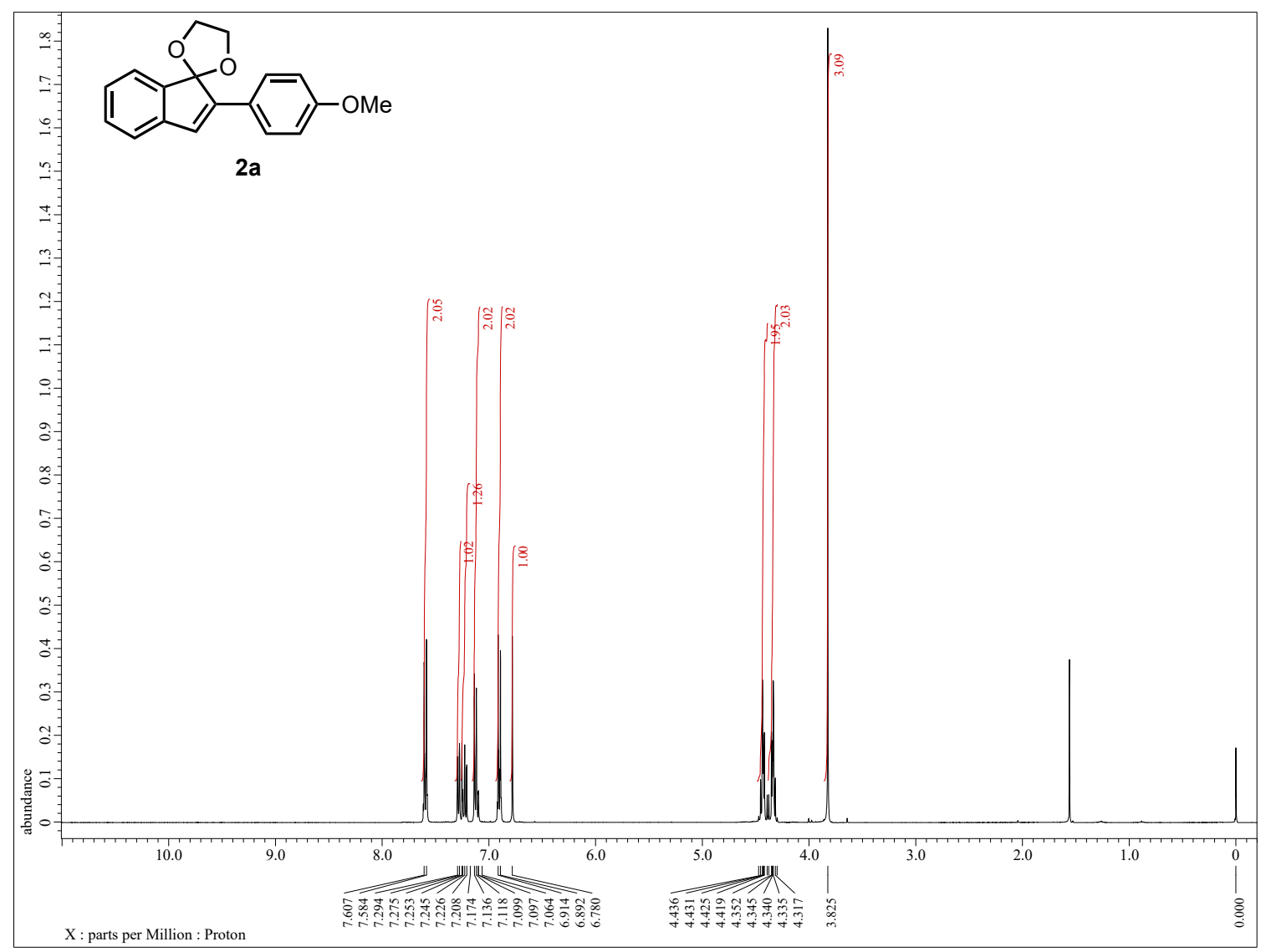

${ }^{13} \mathrm{C} \mathrm{NMR} \quad\left(400 \mathrm{MHz}, \mathrm{CDCl}_{3}\right)$ of $\mathbf{2 a}$

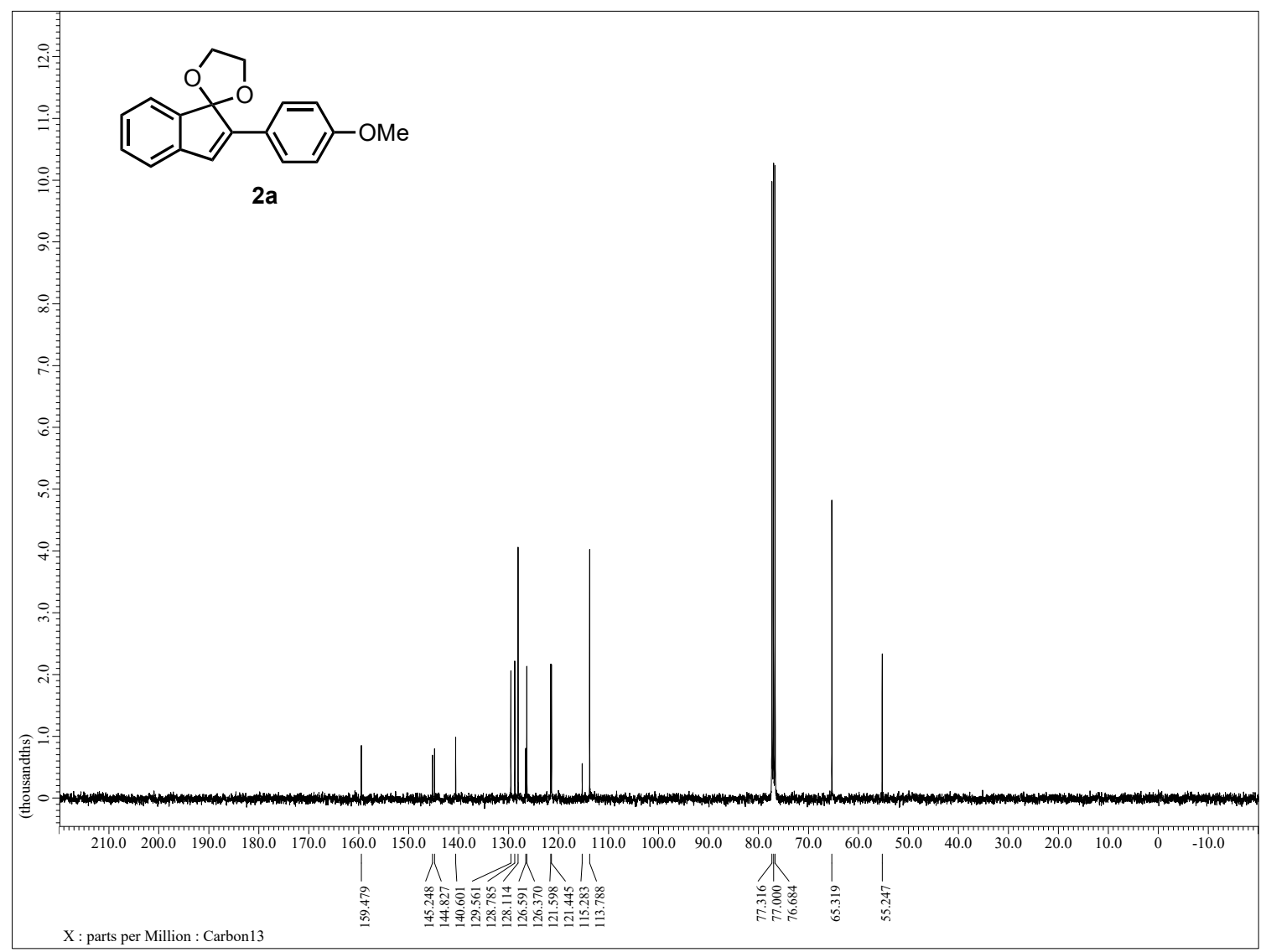


${ }^{1} \mathrm{H} \mathrm{NMR} \quad\left(400 \mathrm{MHz}, \mathrm{CDCl}_{3}\right)$ of $\mathbf{2 b}$

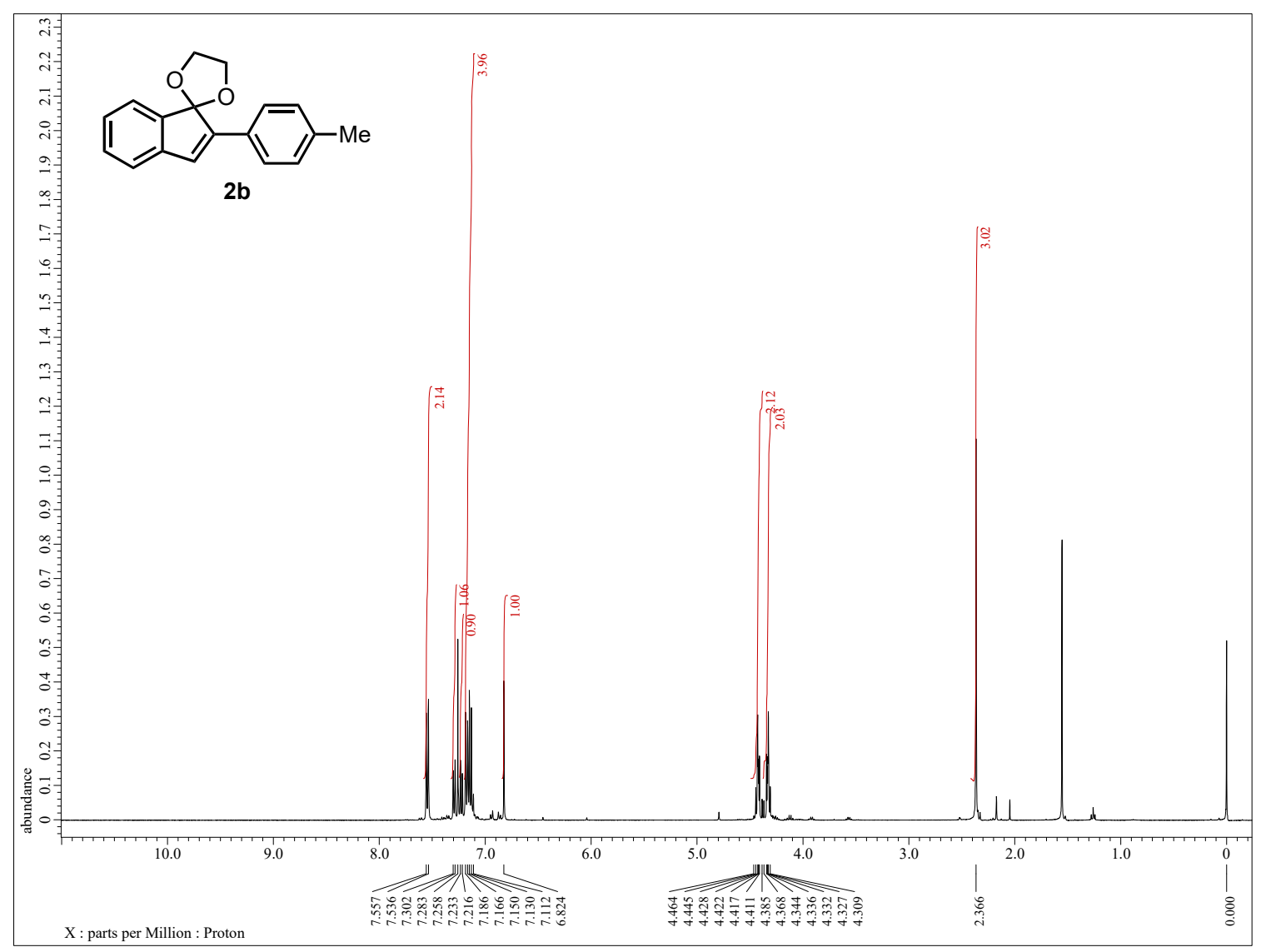

${ }^{13} \mathrm{C}$ NMR $\quad\left(100 \mathrm{MHz}, \mathrm{CDCl}_{3}\right)$ of $\mathbf{2 b}$

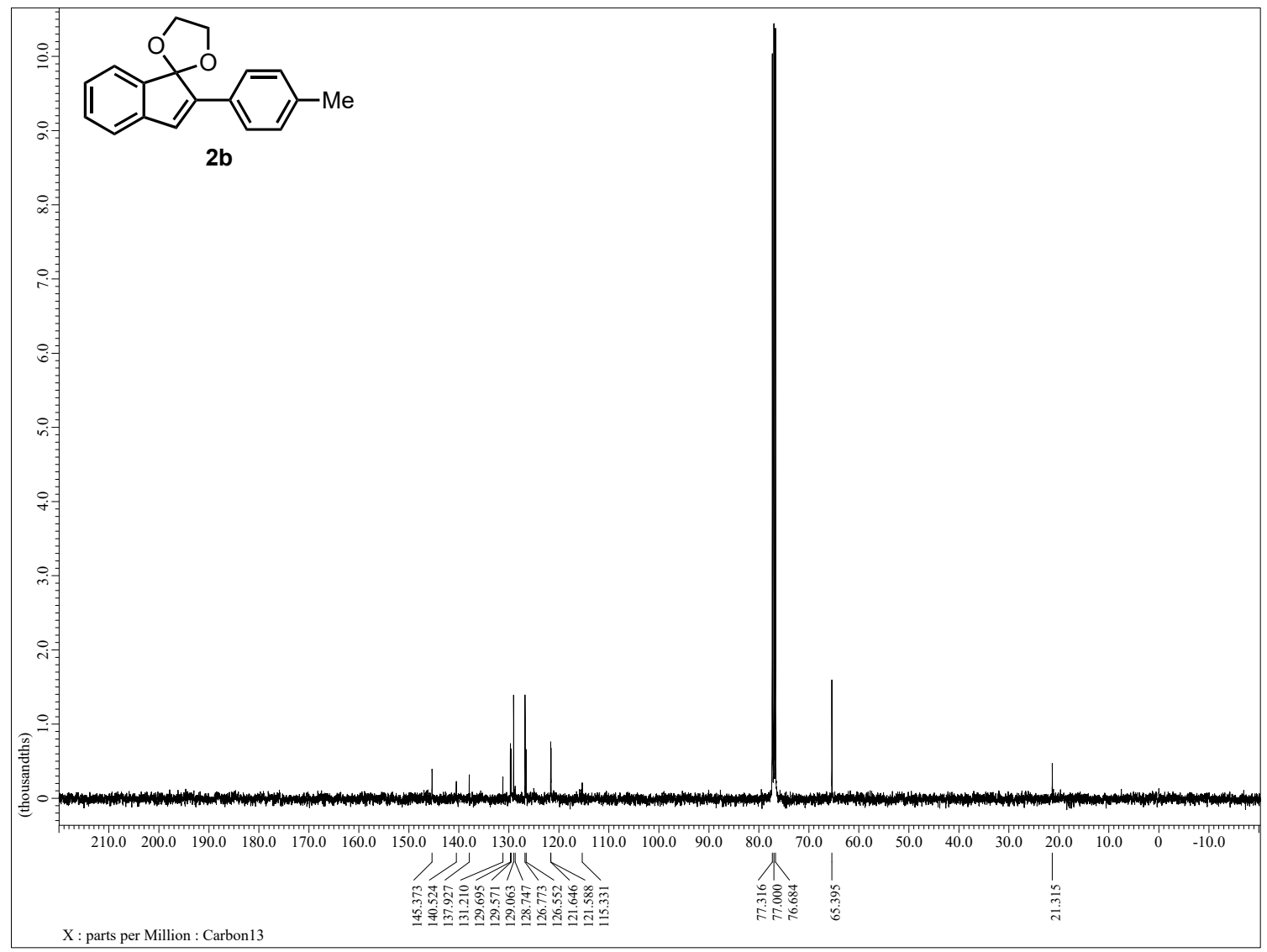


${ }^{1} \mathrm{H}$ NMR $\quad\left(400 \mathrm{MHz}, \mathrm{CDCl}_{3}\right)$ of $\mathbf{2 c}$

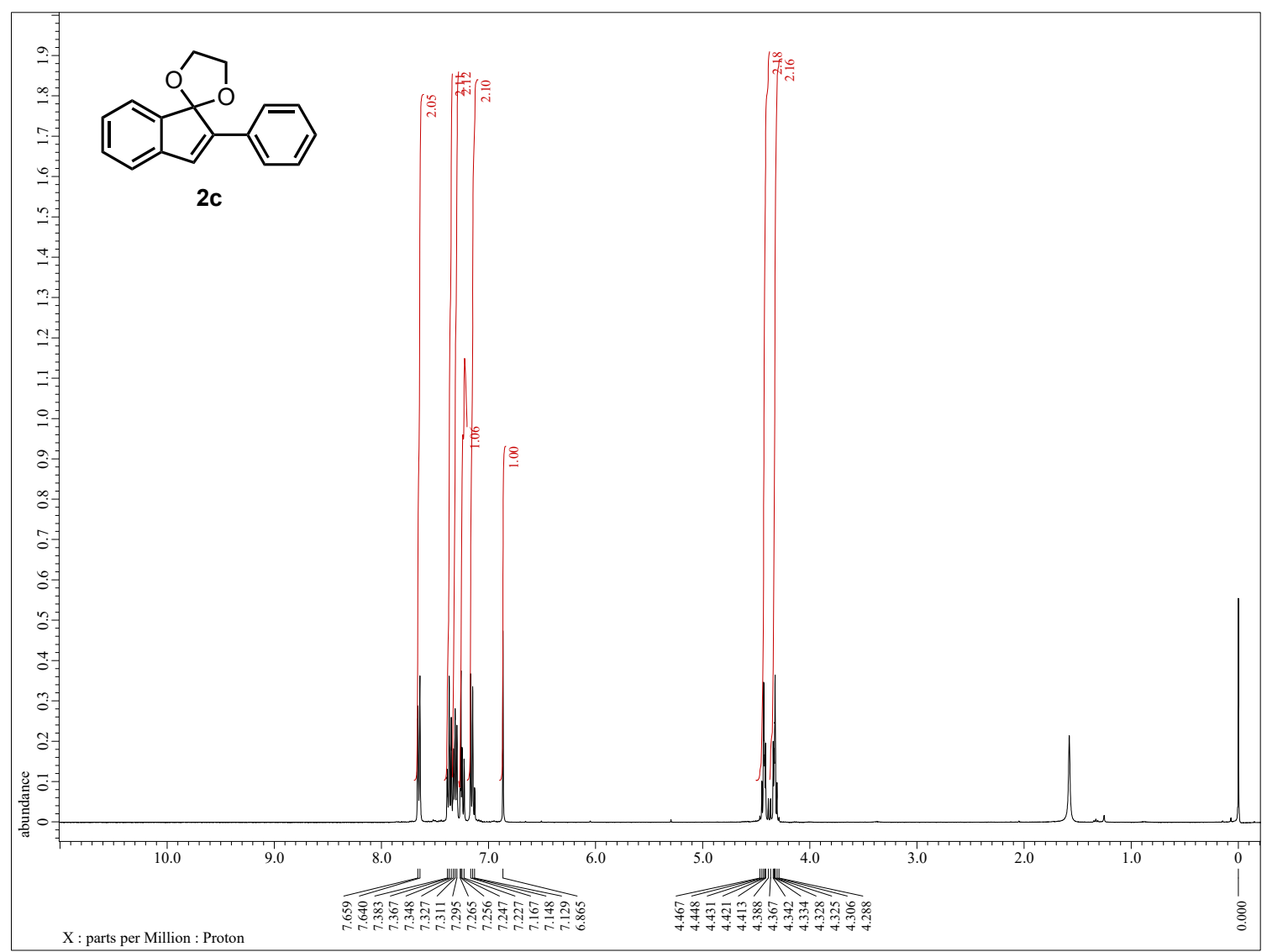

${ }^{13} \mathrm{C} \mathrm{NMR} \quad\left(100 \mathrm{MHz}, \mathrm{CDCl}_{3}\right)$ of $\mathbf{2 c}$

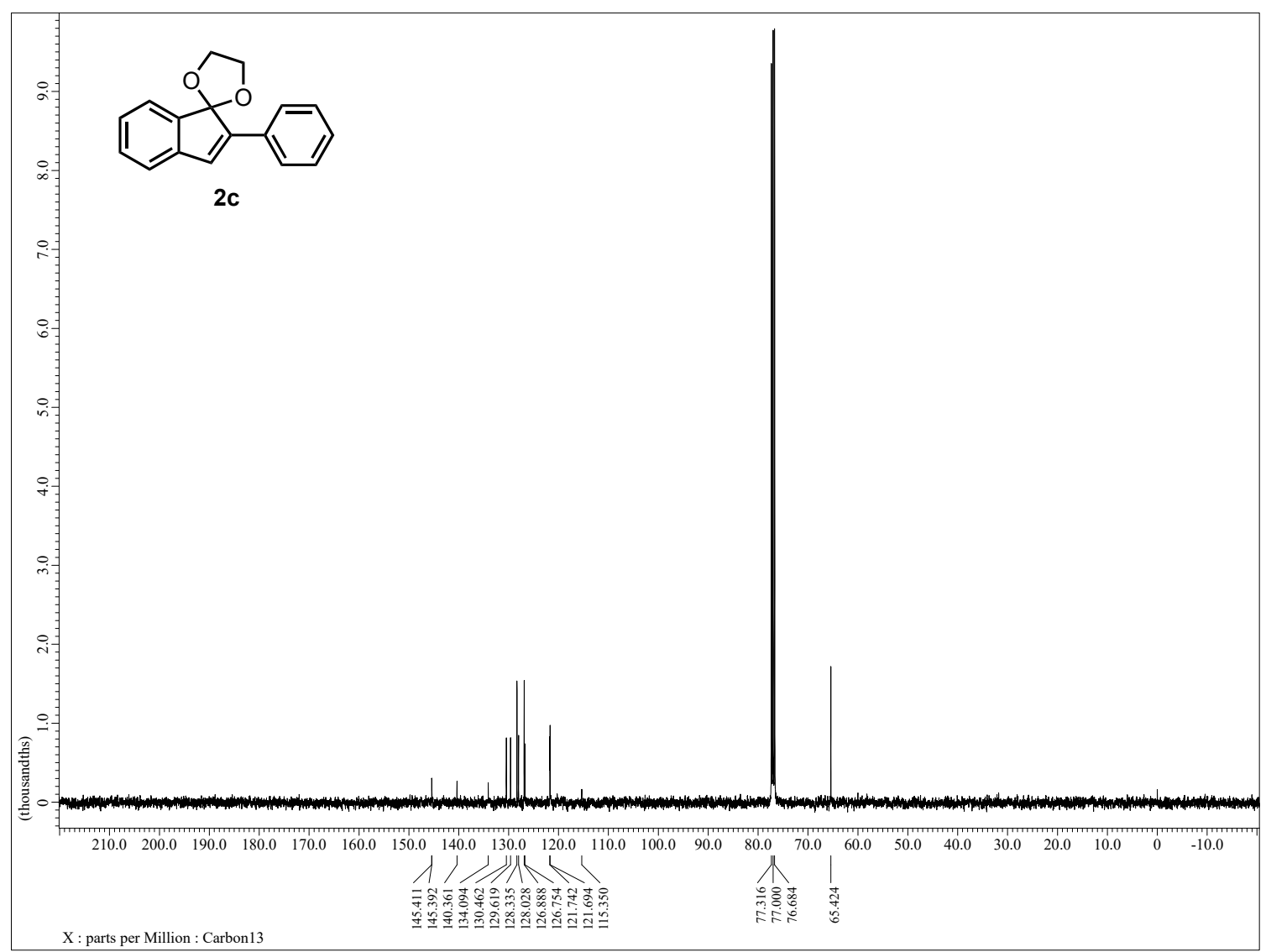


${ }^{1} \mathrm{H}$ NMR (400 MHz, $\mathrm{CDCl}_{3}$ ) of $\mathbf{2 d}$

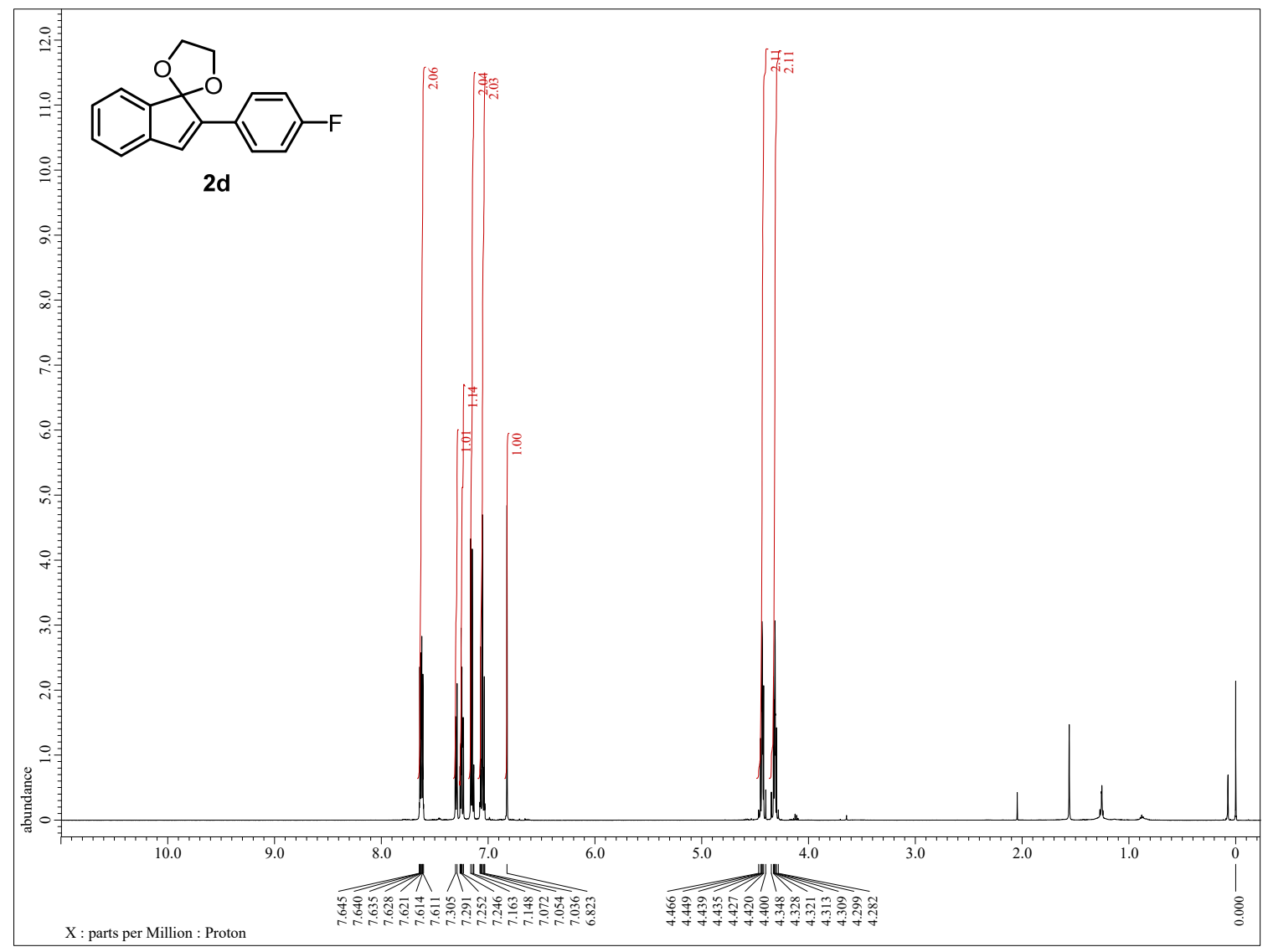

${ }^{13} \mathrm{C}$ NMR $\left(100 \mathrm{MHz}, \mathrm{CDCl}_{3}\right)$ of $\mathbf{2 d}$

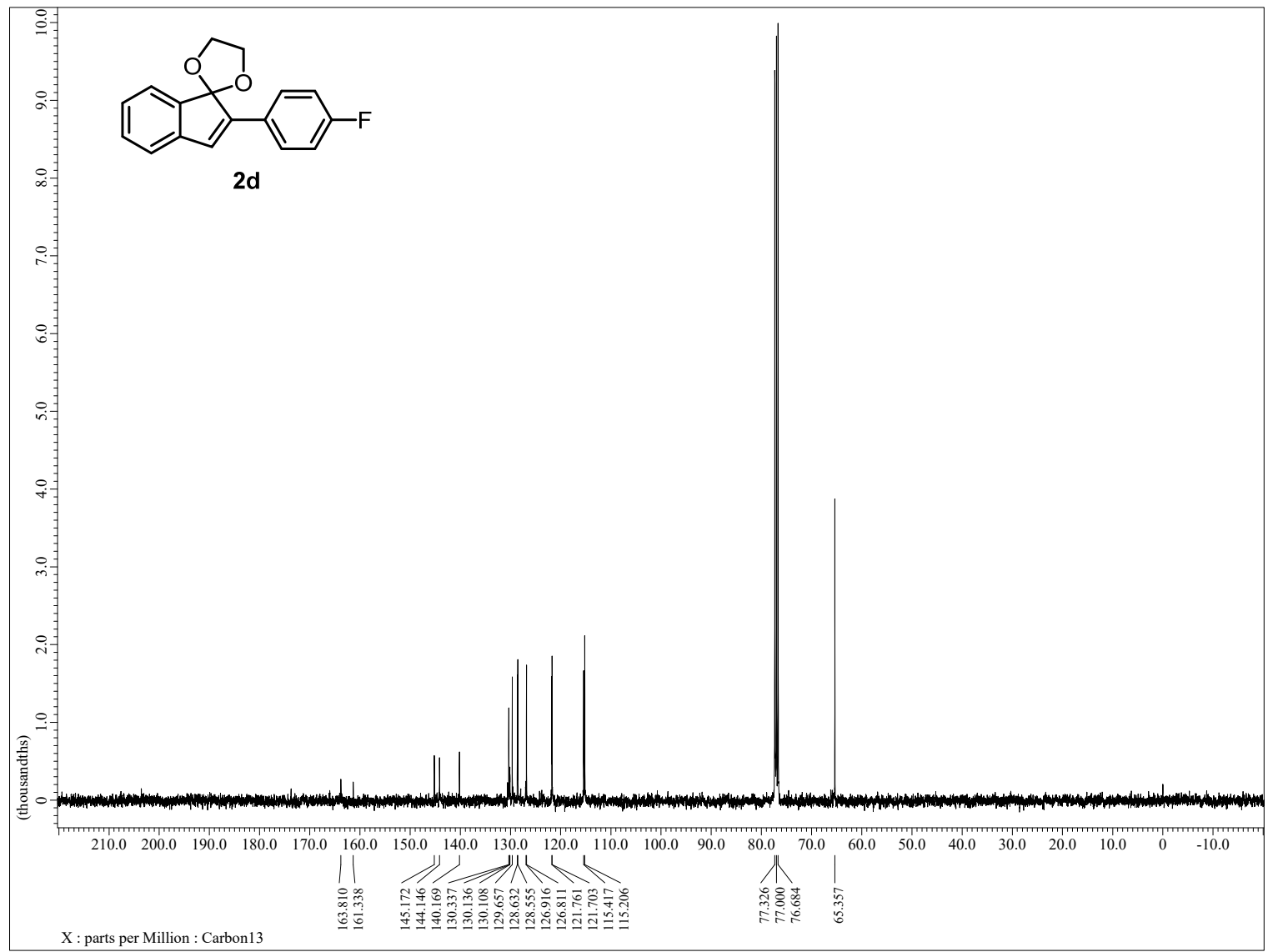


${ }^{1} \mathrm{H}$ NMR $\quad\left(400 \mathrm{MHz}, \mathrm{CDCl}_{3}\right)$ of $\mathbf{2 e}$

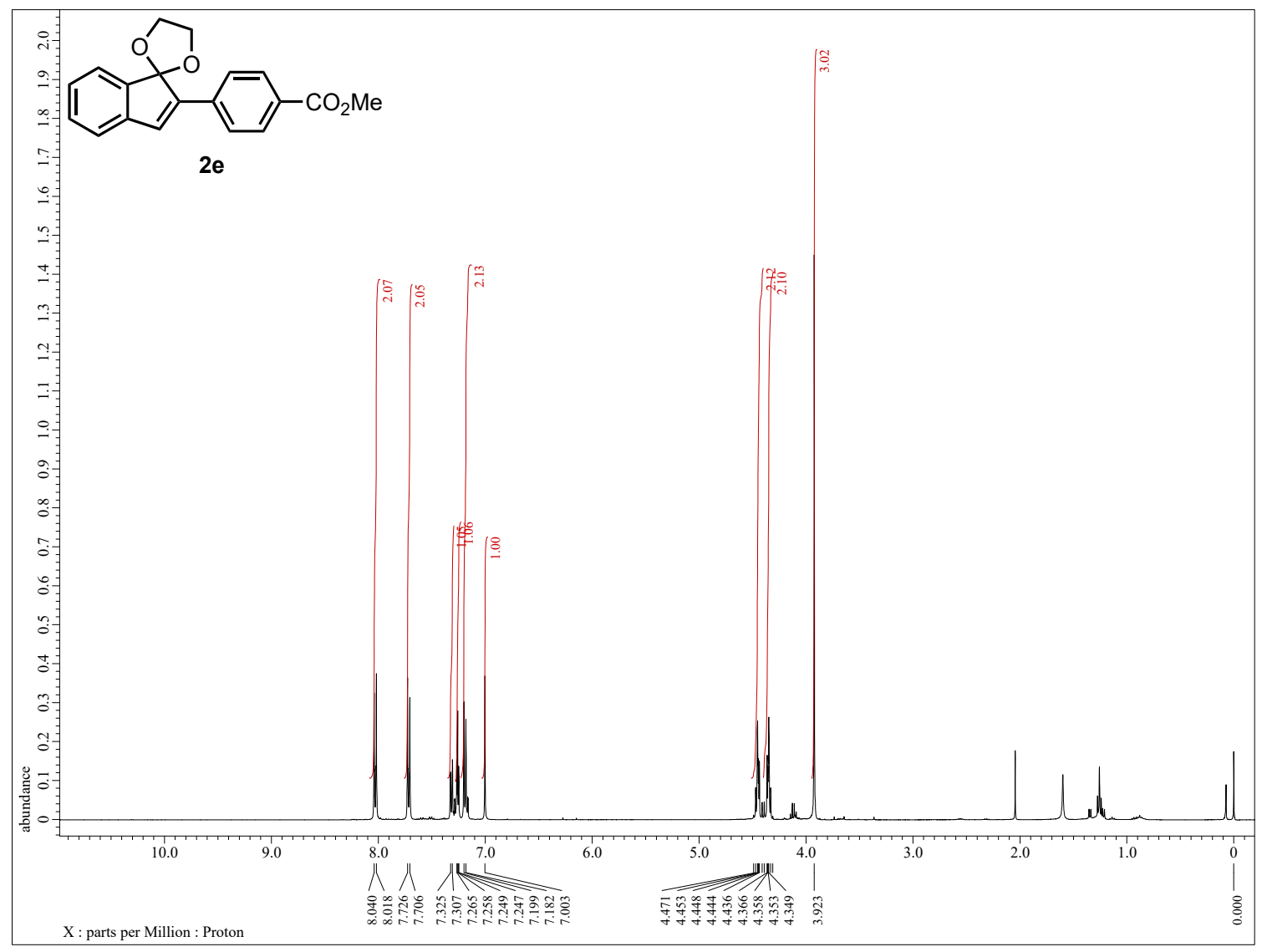

${ }^{13} \mathrm{C} \mathrm{NMR} \quad\left(100 \mathrm{MHz}, \mathrm{CDCl}_{3}\right)$ of $\mathbf{2 c}$

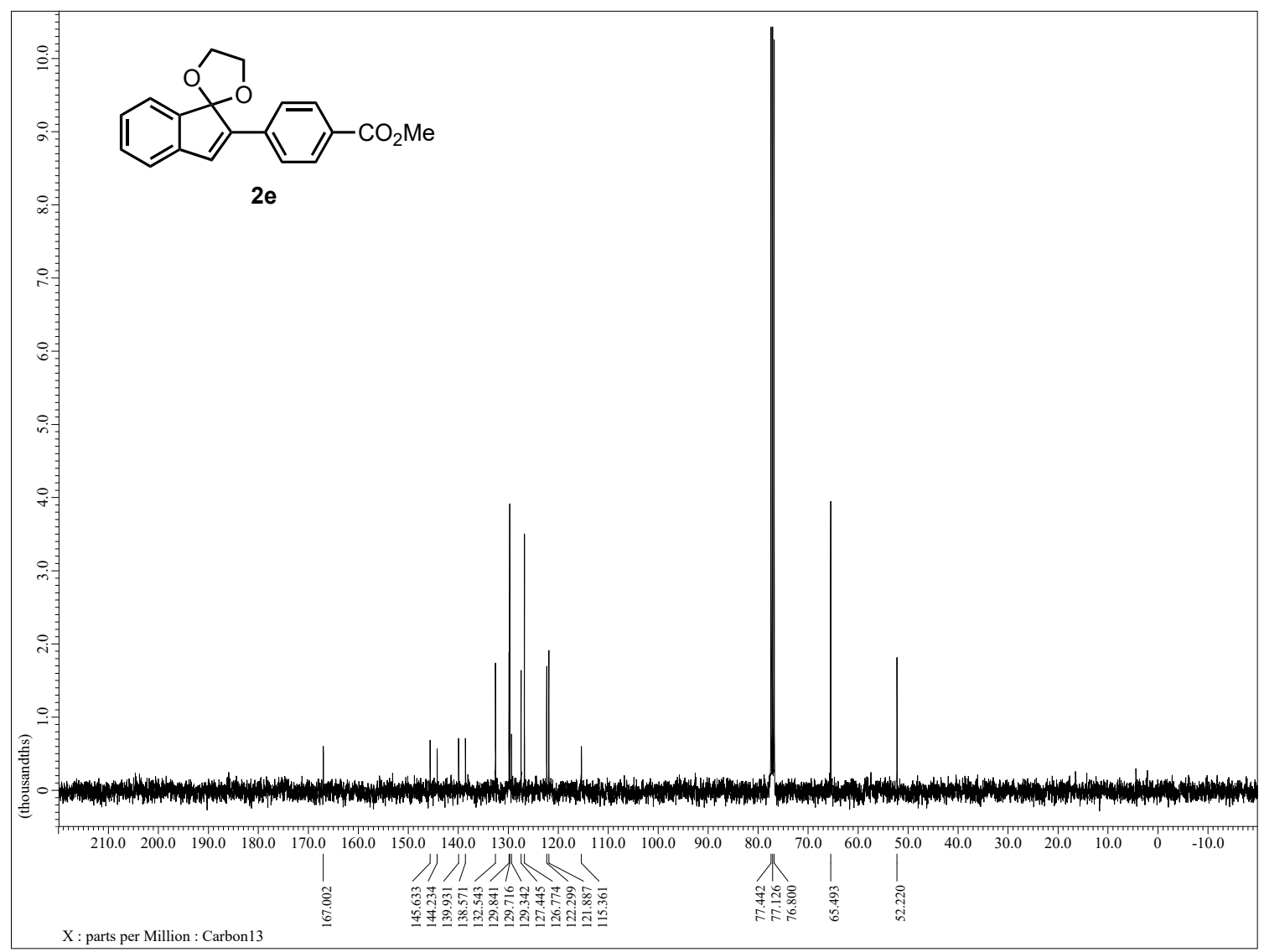


${ }^{1} \mathrm{H}$ NMR $\quad\left(400 \mathrm{MHz}, \mathrm{CDCl}_{3}\right)$ of $\mathbf{2 f}$

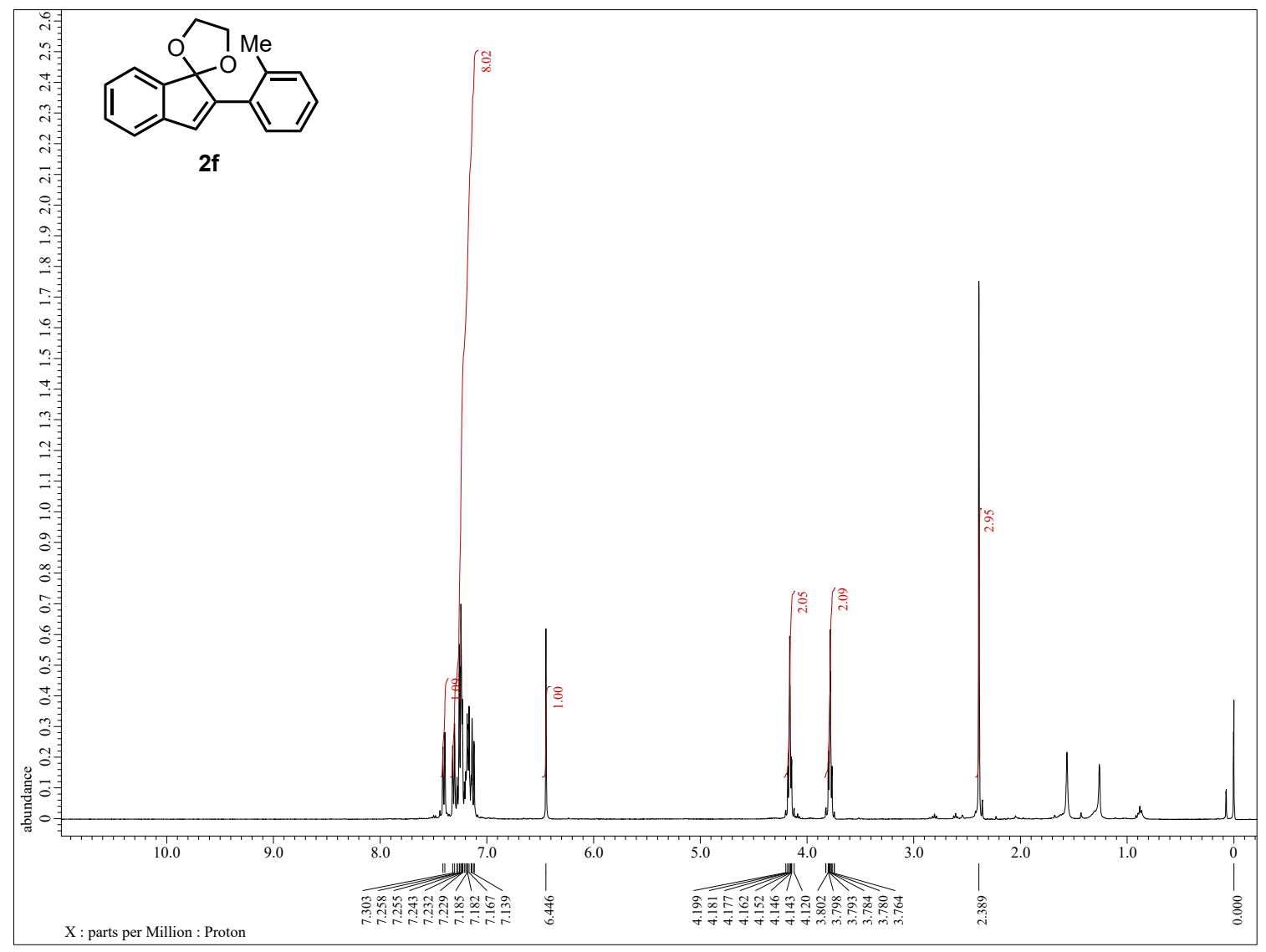

${ }^{13} \mathrm{C} \mathrm{NMR} \quad\left(100 \mathrm{MHz}, \mathrm{CDCl}_{3}\right)$ of $\mathbf{2 f}$

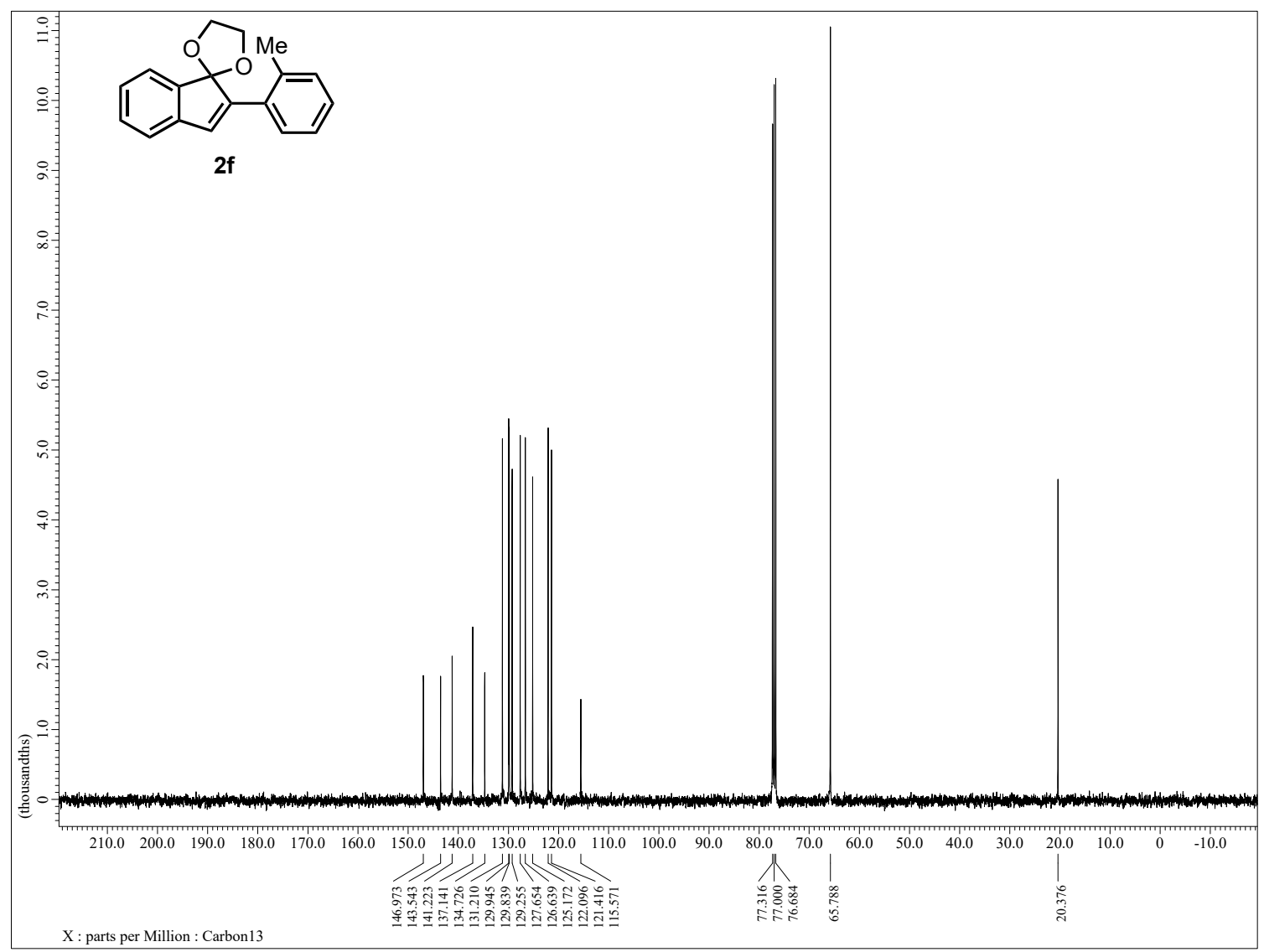


${ }^{1} \mathrm{H} \mathrm{NMR} \quad\left(400 \mathrm{MHz}, \mathrm{CDCl}_{3}\right)$ of $\mathbf{2 g}$

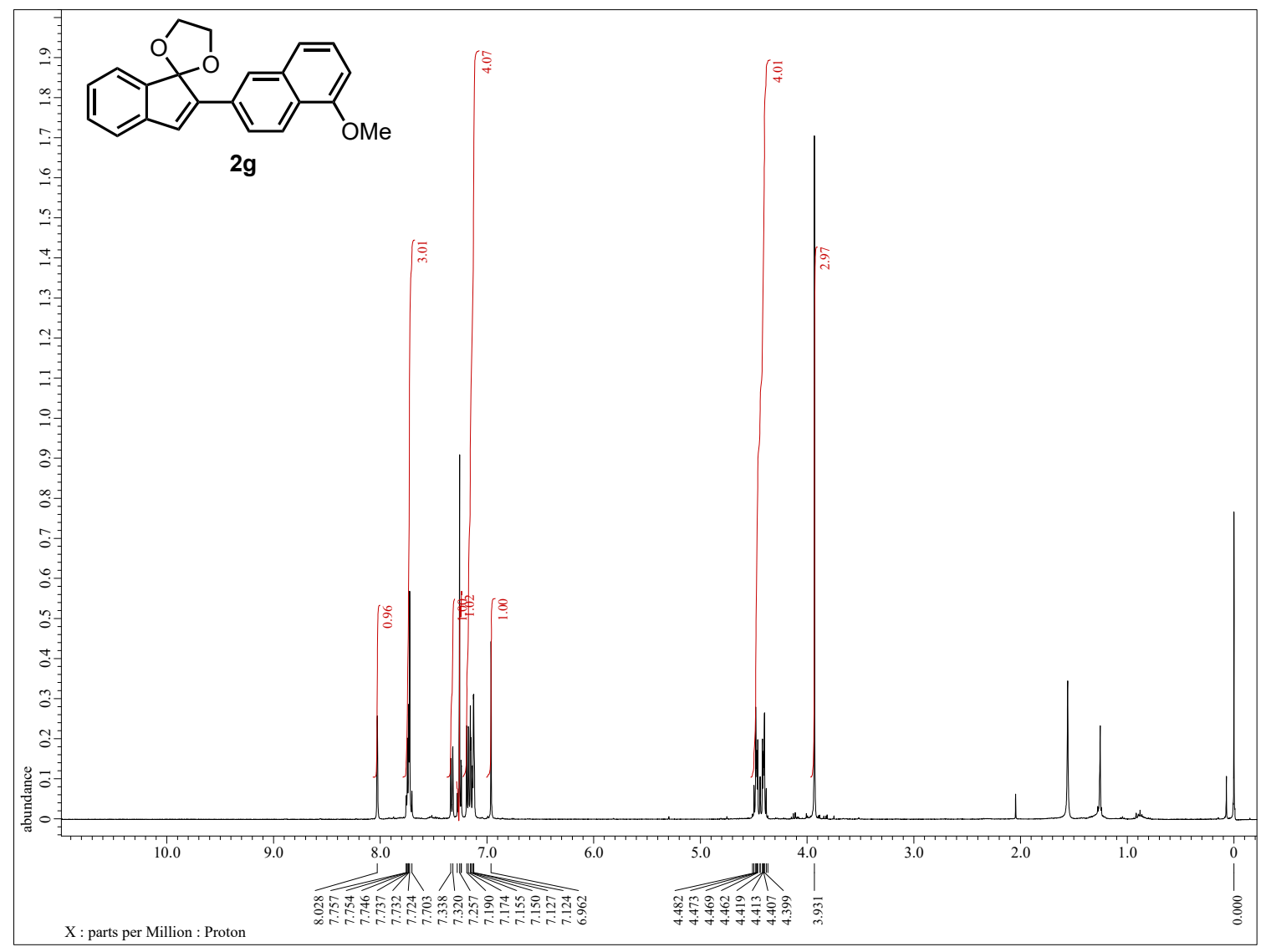

${ }^{13} \mathrm{C}$ NMR $\quad\left(100 \mathrm{MHz}, \mathrm{CDCl}_{3}\right)$ of $\mathbf{2 g}$

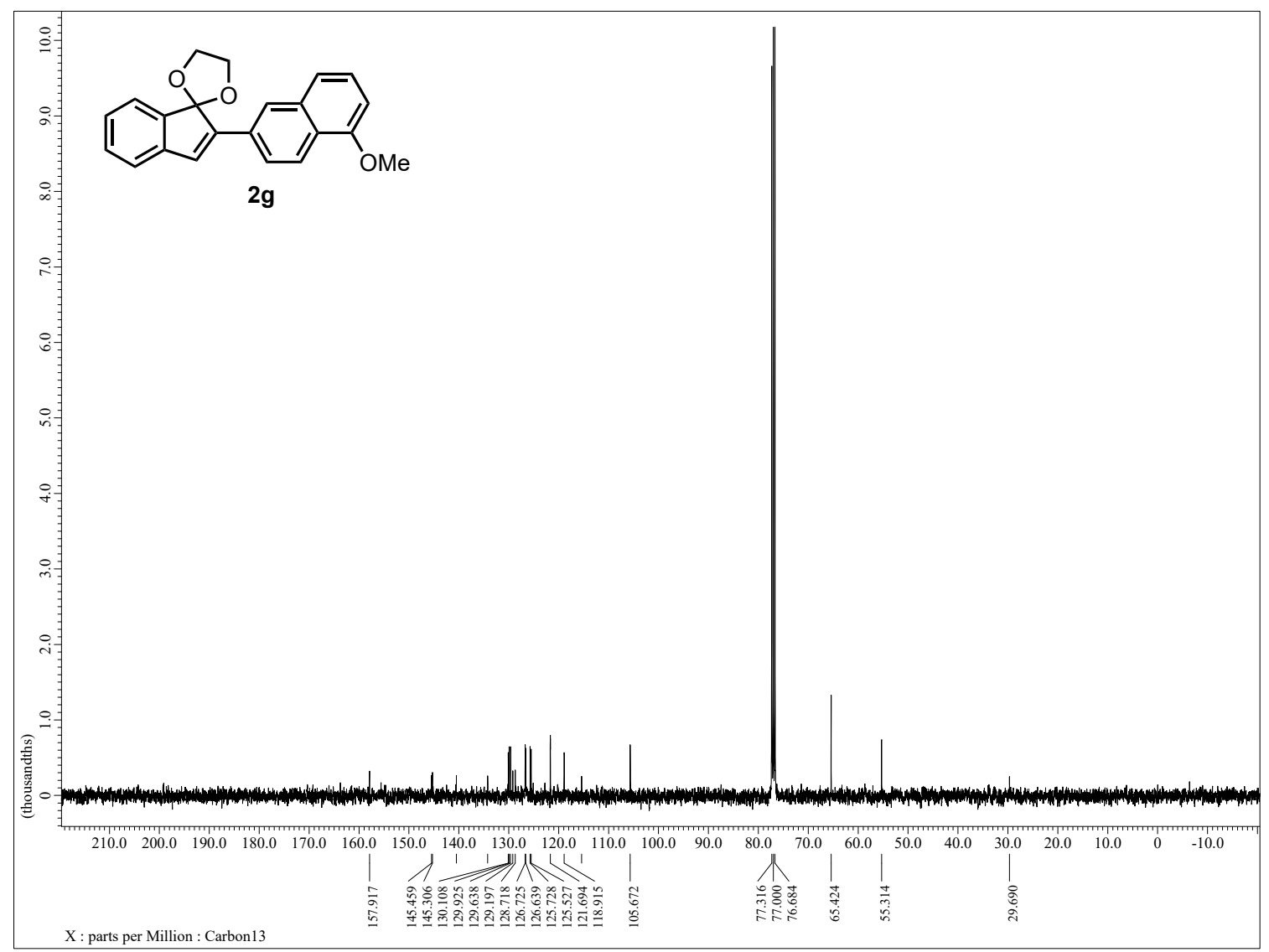


${ }^{1} \mathrm{H}$ NMR $\quad\left(400 \mathrm{MHz}, \mathrm{CDCl}_{3}\right)$ of $\mathbf{2 h}$

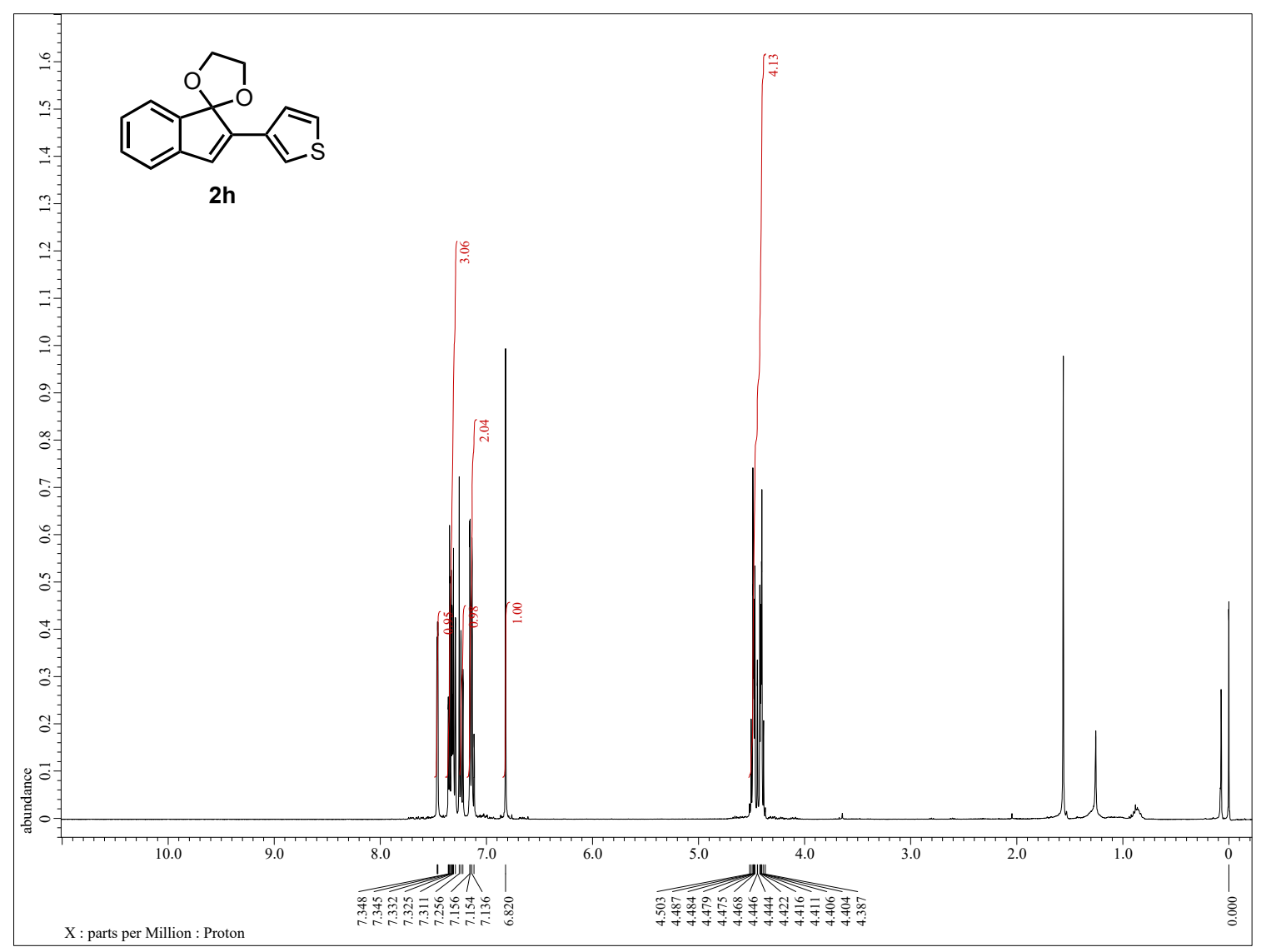

${ }^{13} \mathrm{C}$ NMR $\quad\left(100 \mathrm{MHz}, \mathrm{CDCl}_{3}\right)$ of $\mathbf{2 h}$

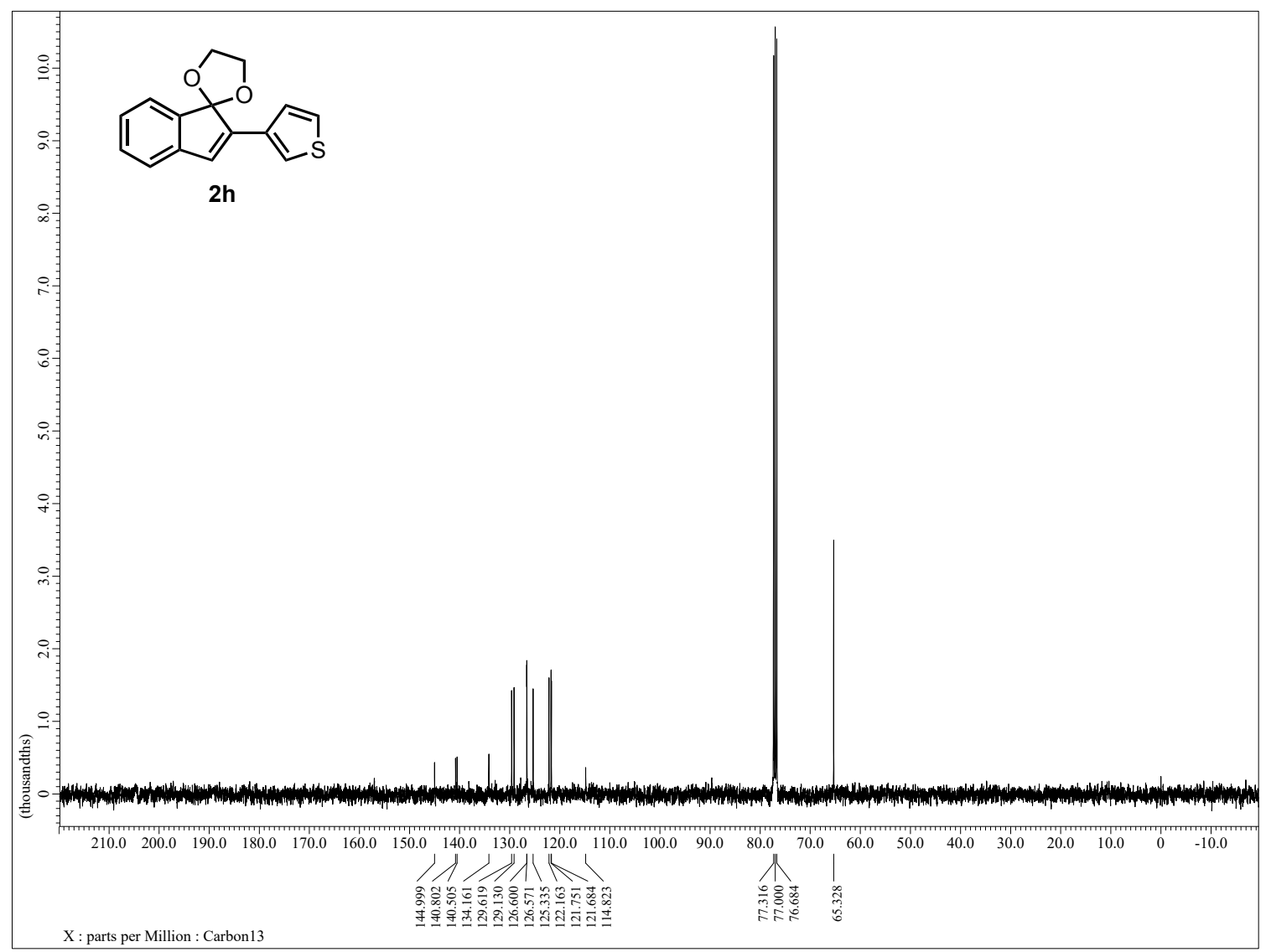


${ }^{1} \mathrm{H}$ NMR $\quad\left(400 \mathrm{MHz}, \mathrm{CDCl}_{3}\right)$ of $\mathbf{2 i}$

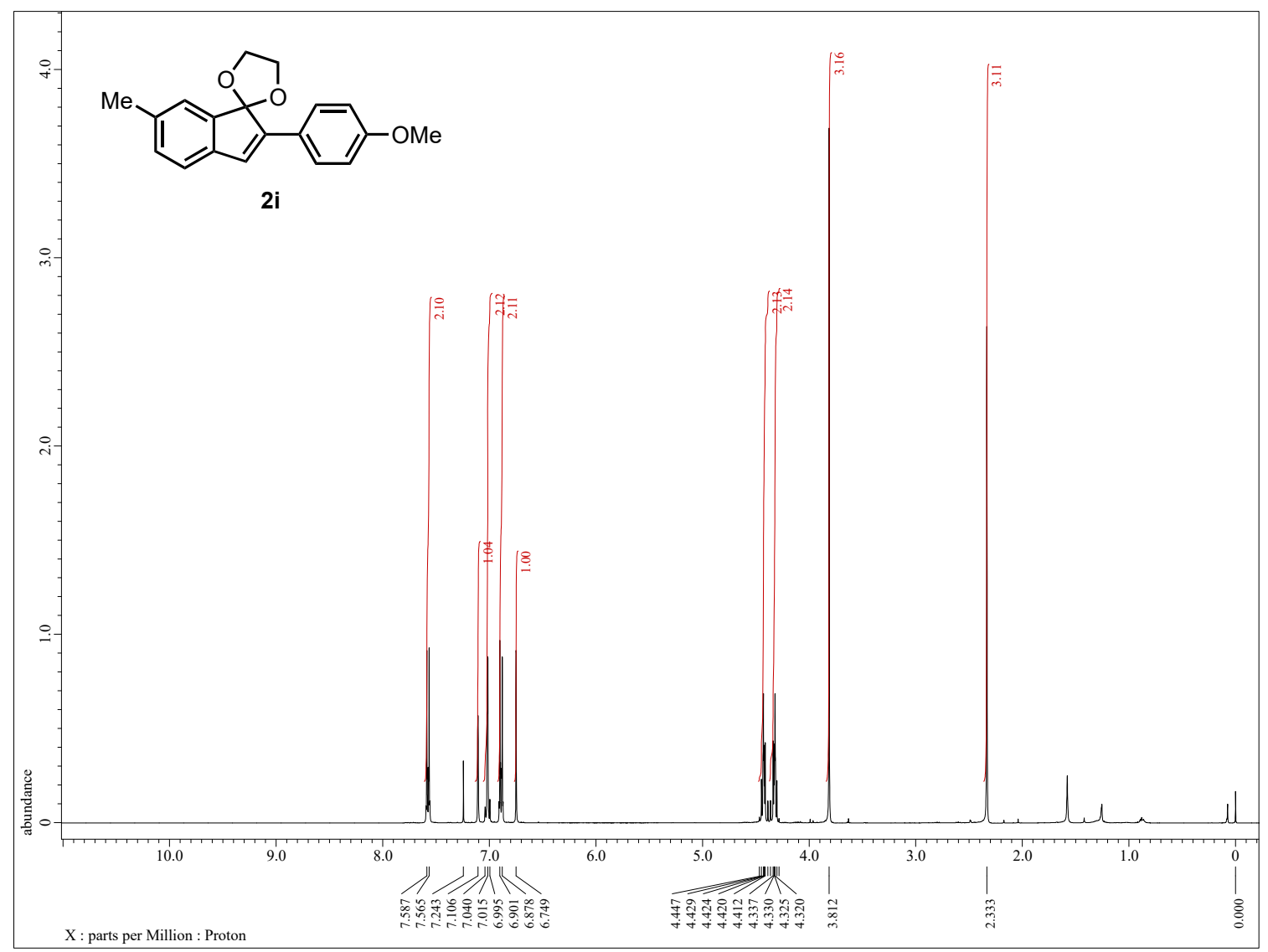

${ }^{13} \mathrm{C}$ NMR $\quad\left(100 \mathrm{MHz}, \mathrm{CDCl}_{3}\right)$ of $\mathbf{2 i}$

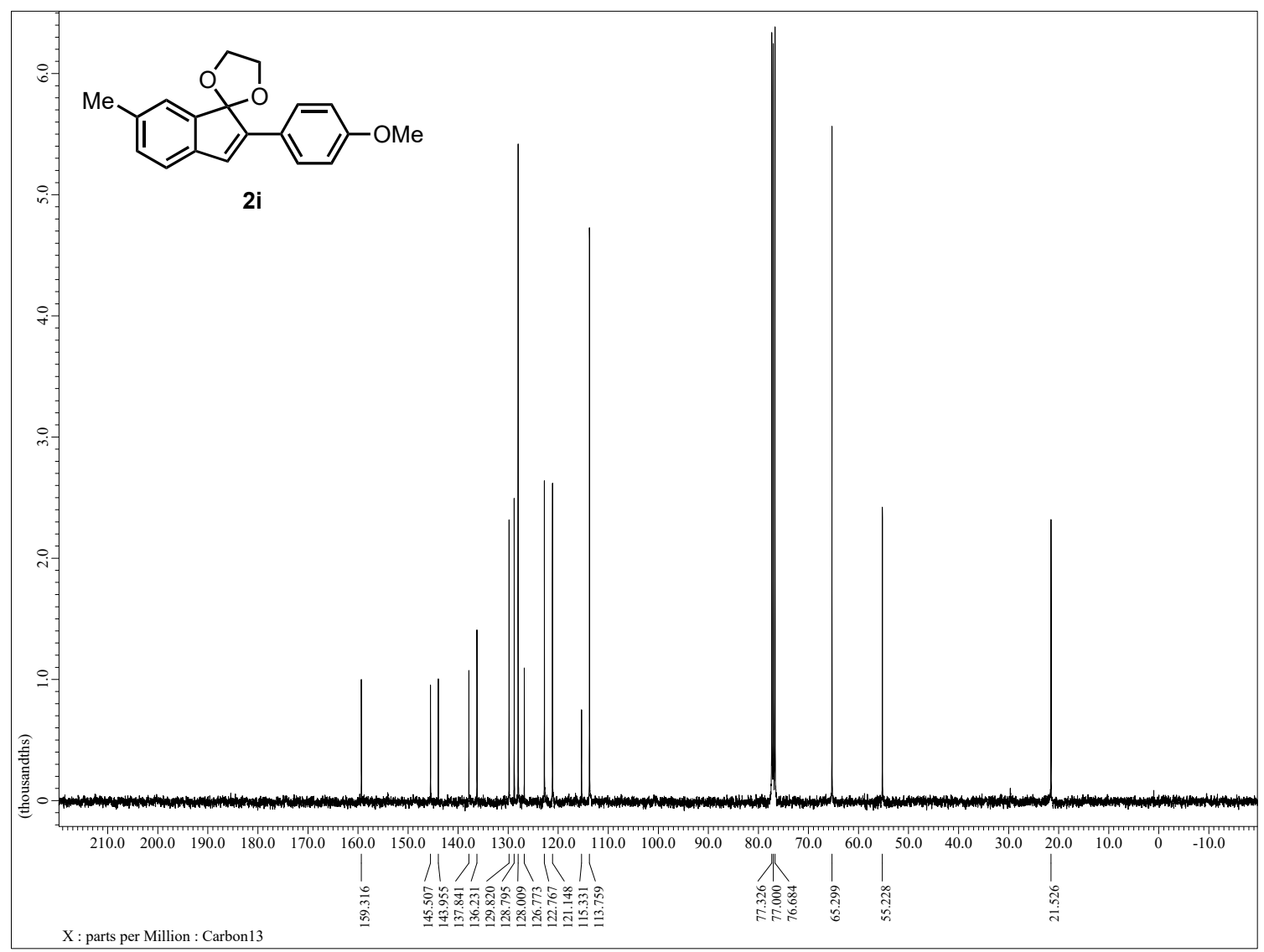


${ }^{1} \mathrm{H}$ NMR $\quad\left(400 \mathrm{MHz}, \mathrm{CDCl}_{3}\right)$ of $\mathbf{2} \mathbf{j}$

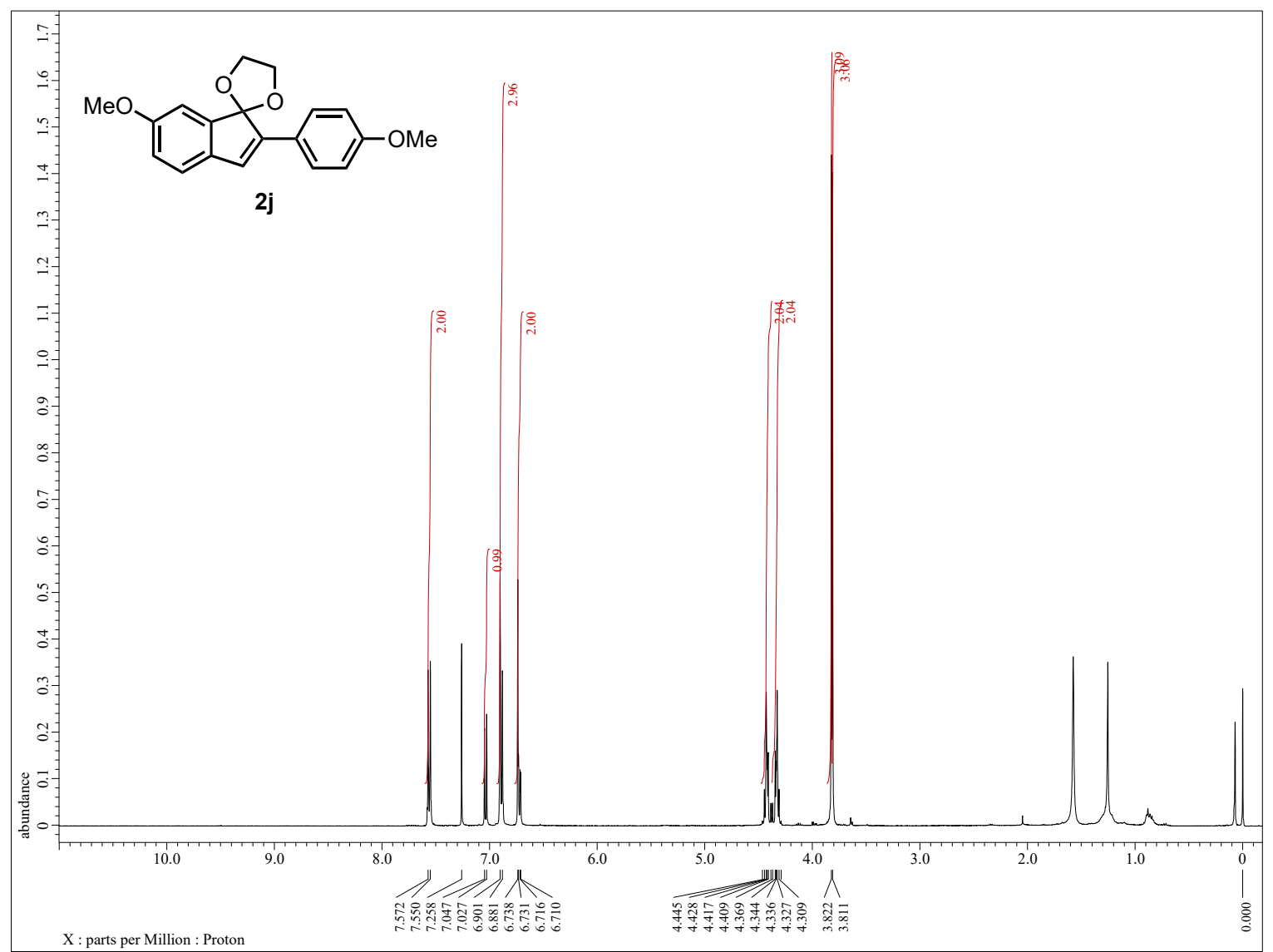

${ }^{13} \mathrm{C}$ NMR $\left(100 \mathrm{MHz}, \mathrm{CDCl}_{3}\right)$ of $\mathbf{2} \mathbf{j}$

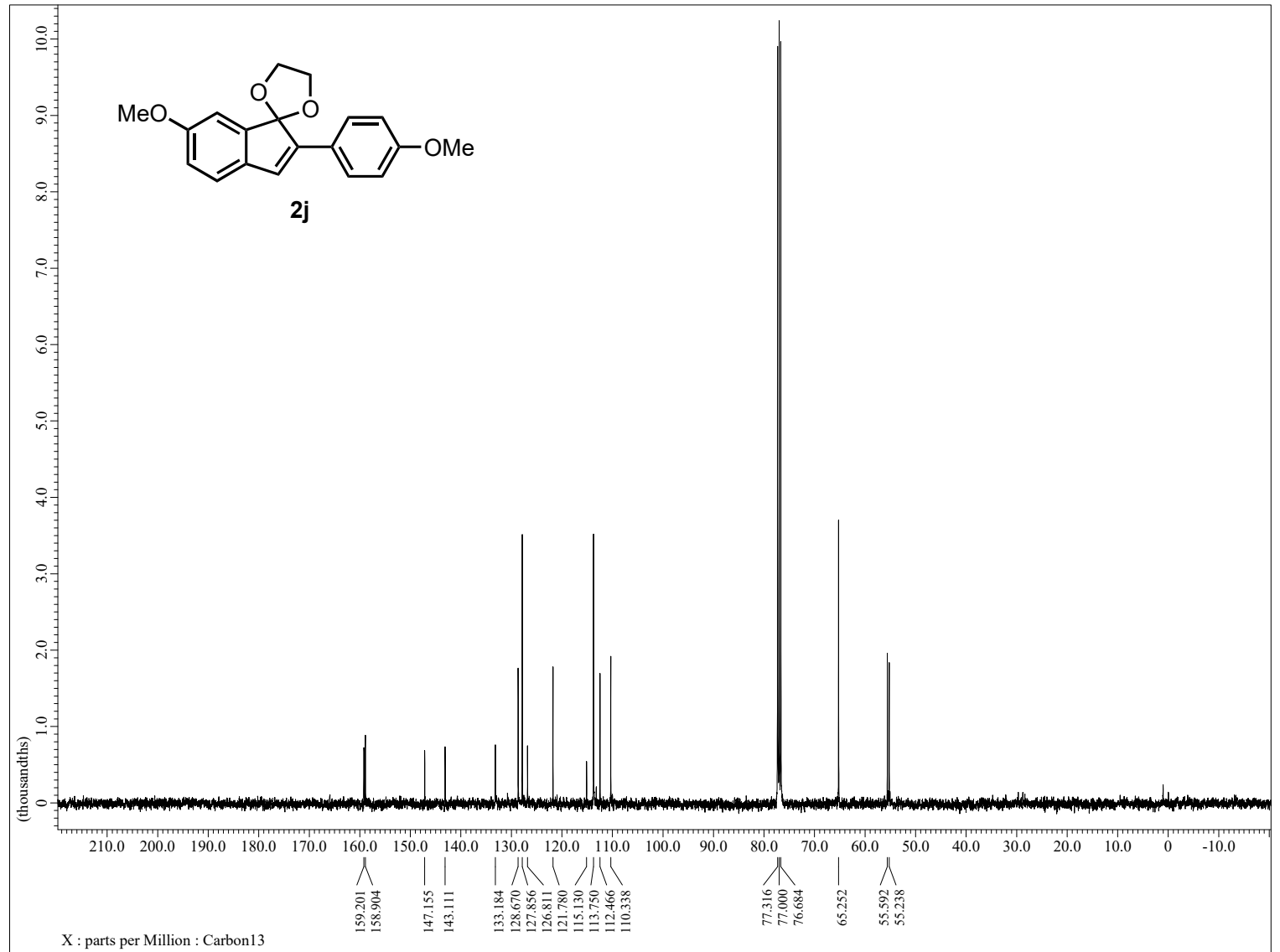


${ }^{1} \mathrm{H}$ NMR $\quad\left(400 \mathrm{MHz}, \mathrm{CDCl}_{3}\right)$ of $\mathbf{2 k}$

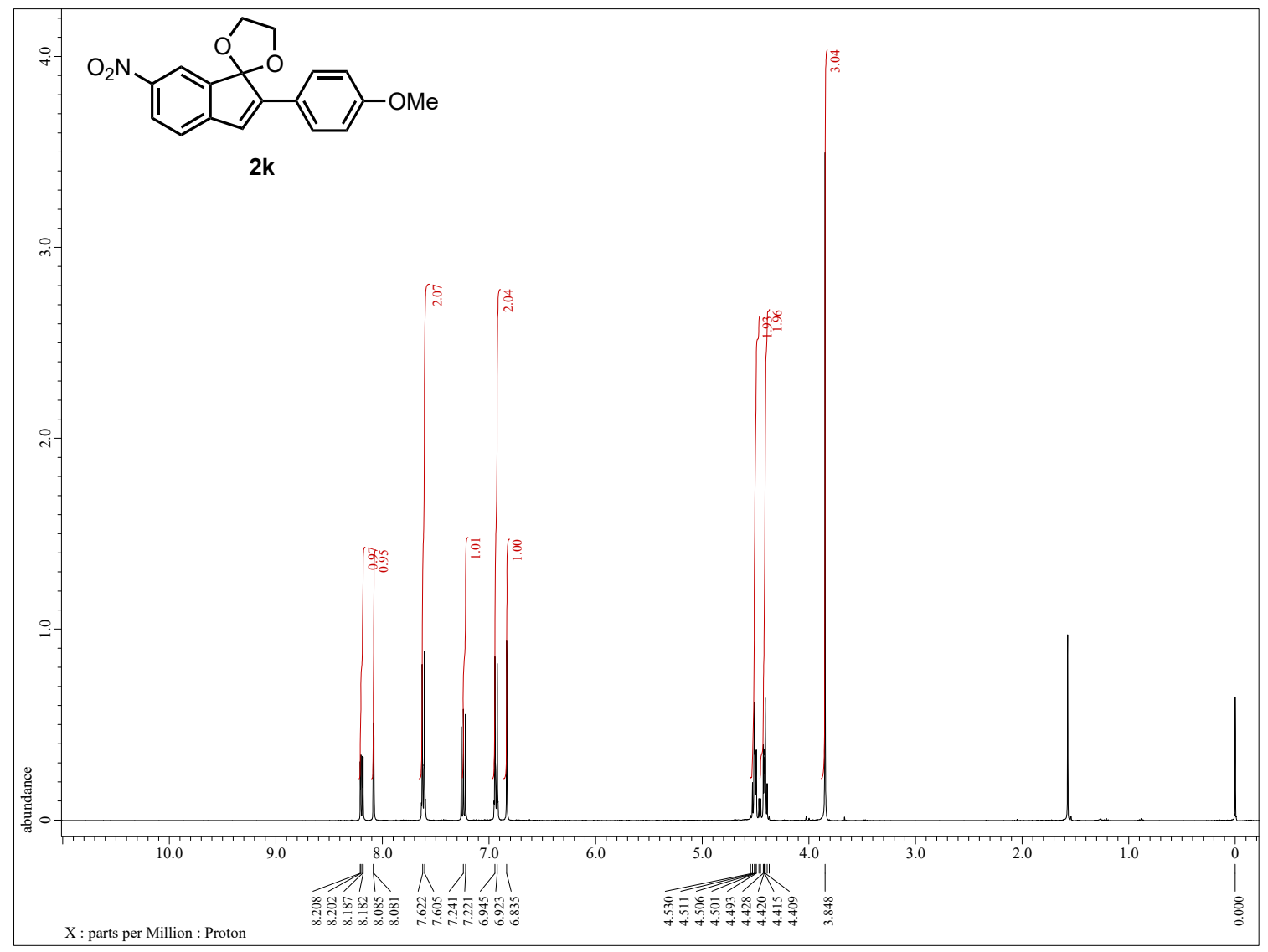

${ }^{13} \mathrm{C}$ NMR $\quad\left(100 \mathrm{MHz}, \mathrm{CDCl}_{3}\right)$ of $\mathbf{2 k}$

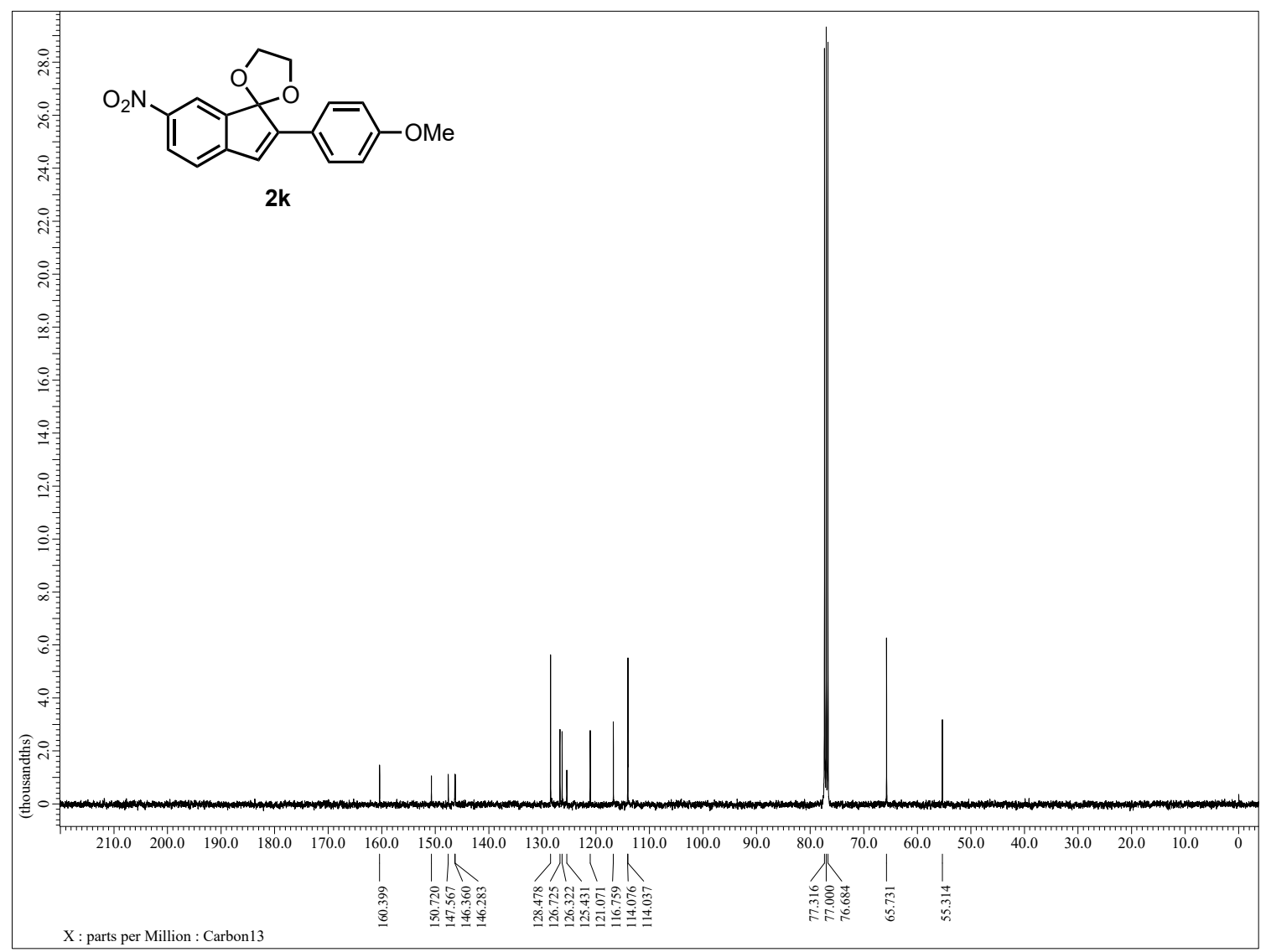


${ }^{1} \mathrm{H}$ NMR $\quad\left(400 \mathrm{MHz}, \mathrm{CDCl}_{3}\right)$ of $\mathbf{2 l}$

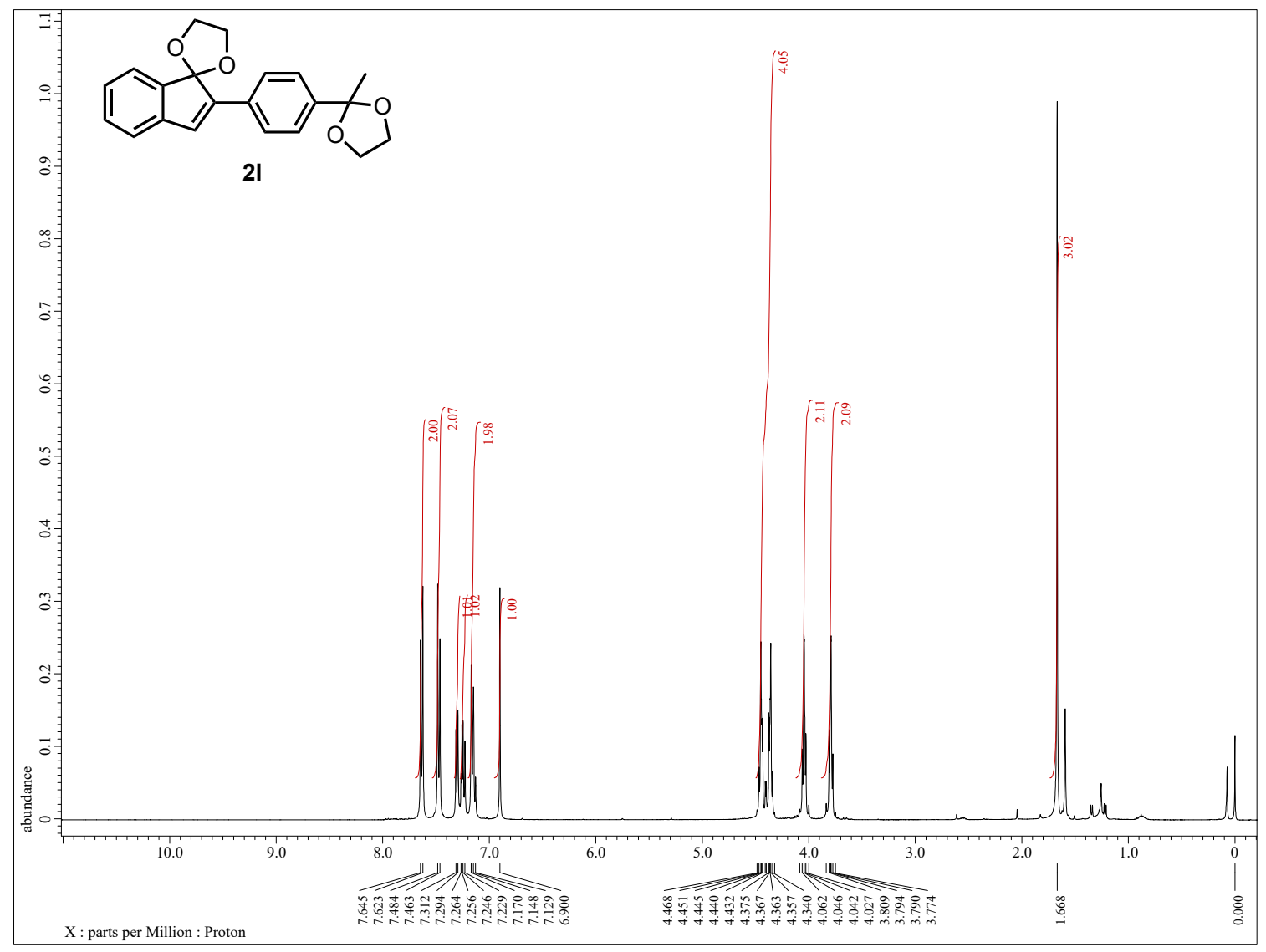

${ }^{13} \mathrm{C}$ NMR $\quad\left(100 \mathrm{MHz}, \mathrm{CDCl}_{3}\right)$ of $\mathbf{2 l}$

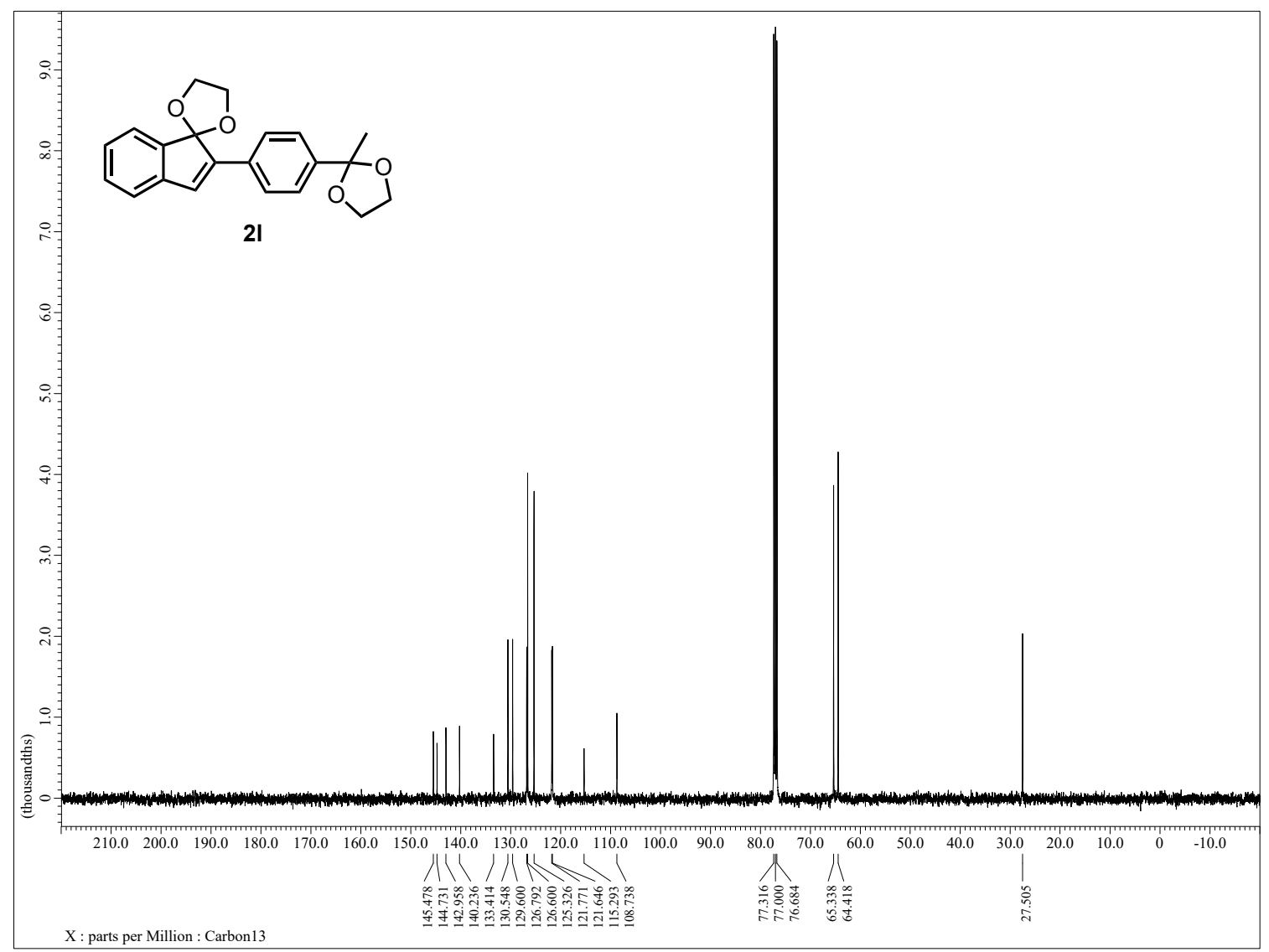


${ }^{1} \mathrm{H}$ NMR (400 MHz, $\mathrm{CDCl}_{4}$ ) of $\mathbf{2 m}$

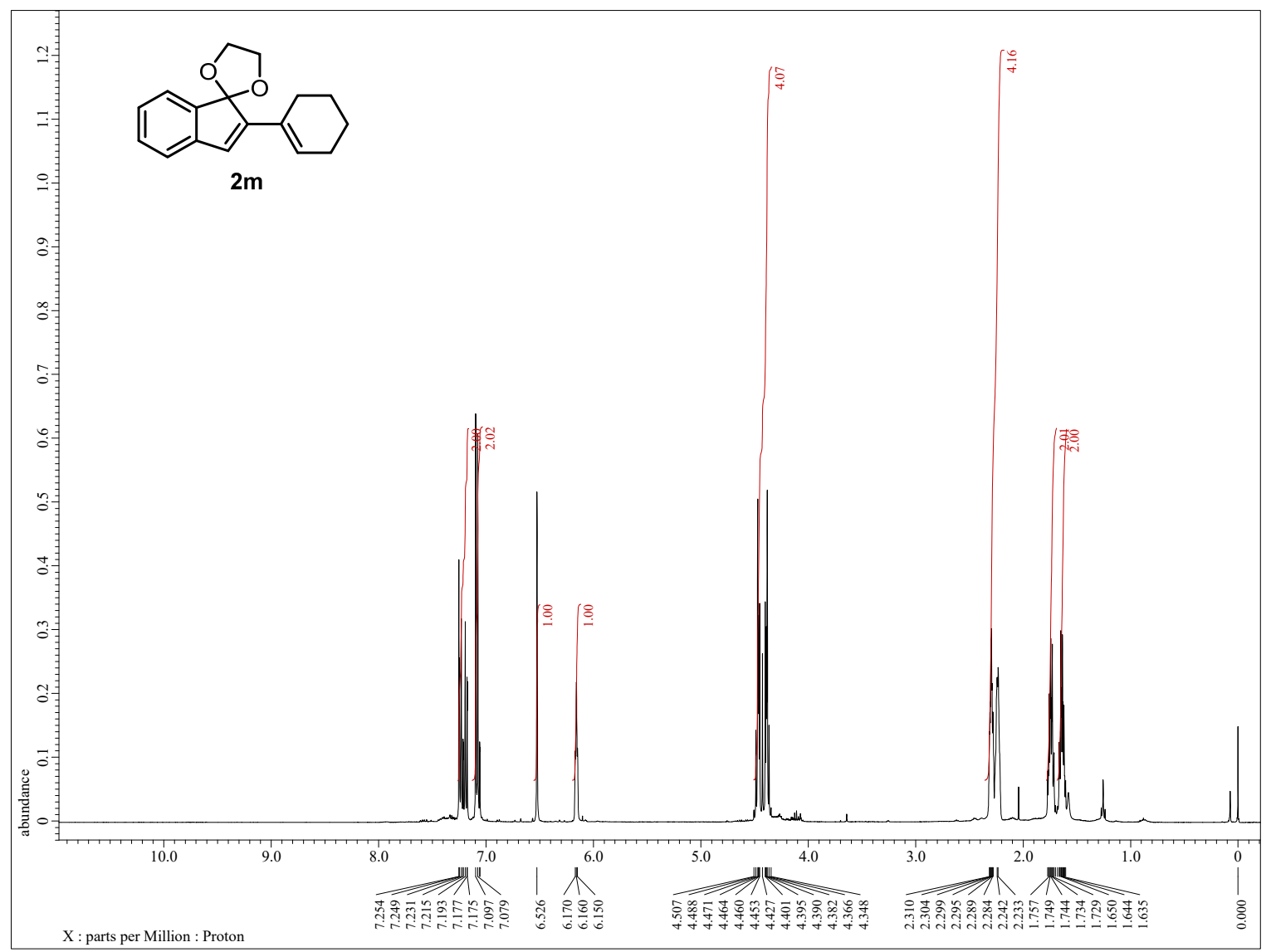

${ }^{13} \mathrm{C}$ NMR (100 MHz, $\mathrm{CDCl}_{3}$ ) of $\mathbf{2 m}$

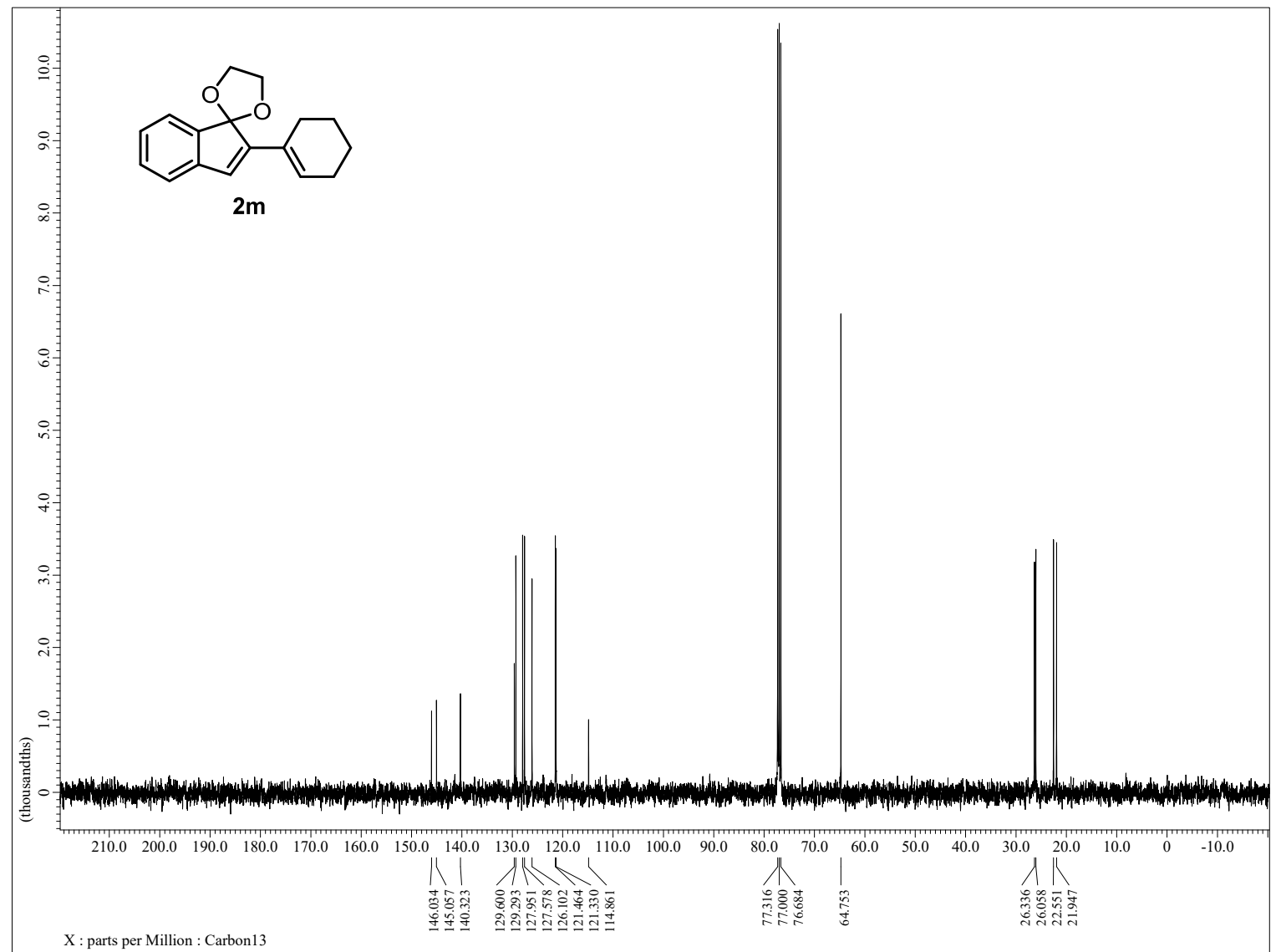

${ }^{1} \mathrm{H} \mathrm{NMR} \quad\left(400 \mathrm{MHz}, \mathrm{CDCl}_{3}\right)$ of $\mathbf{2 n}$ 


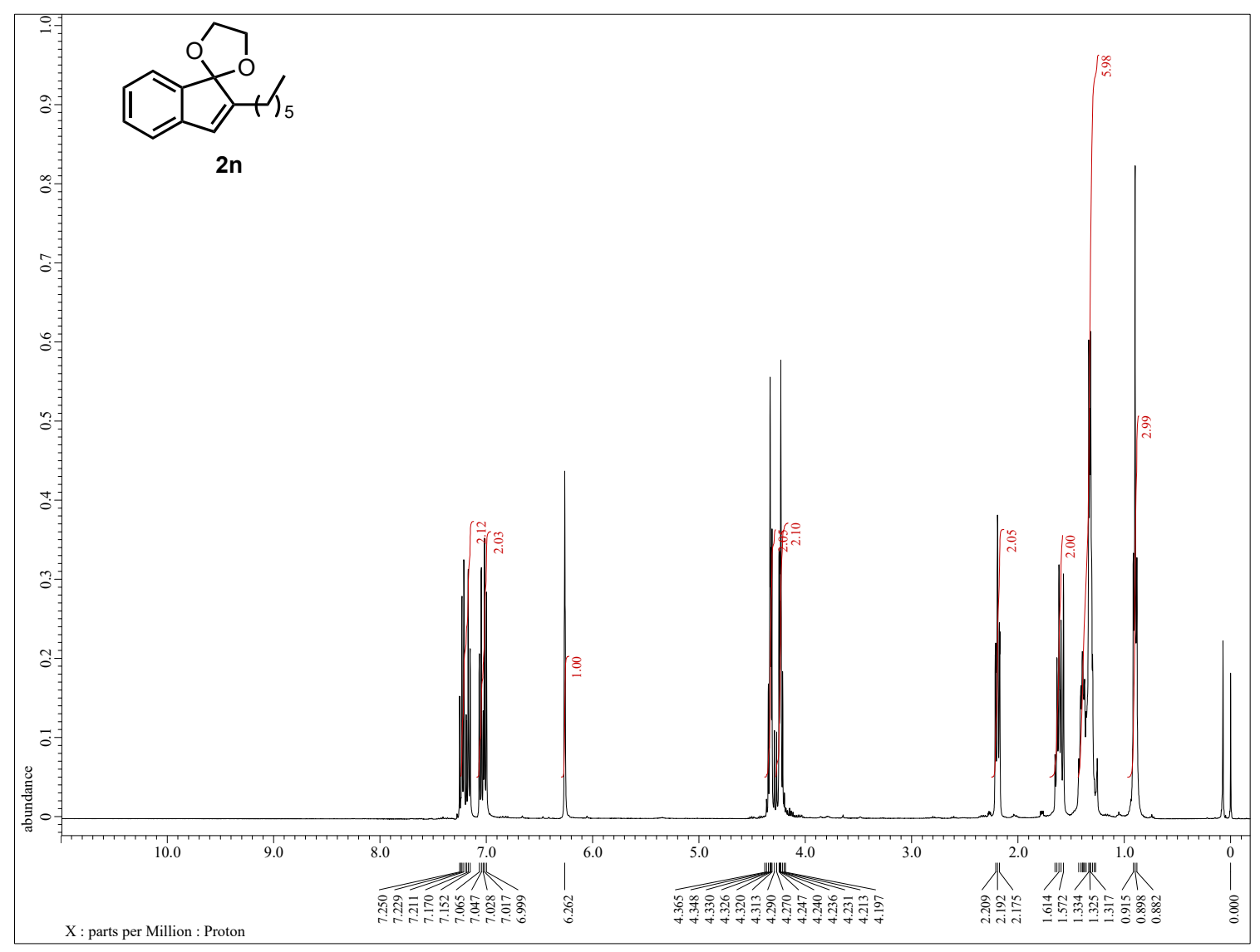

${ }^{13} \mathrm{C}$ NMR $\quad\left(100 \mathrm{MHz}, \mathrm{CDCl}_{3}\right)$ of $\mathbf{2 n}$

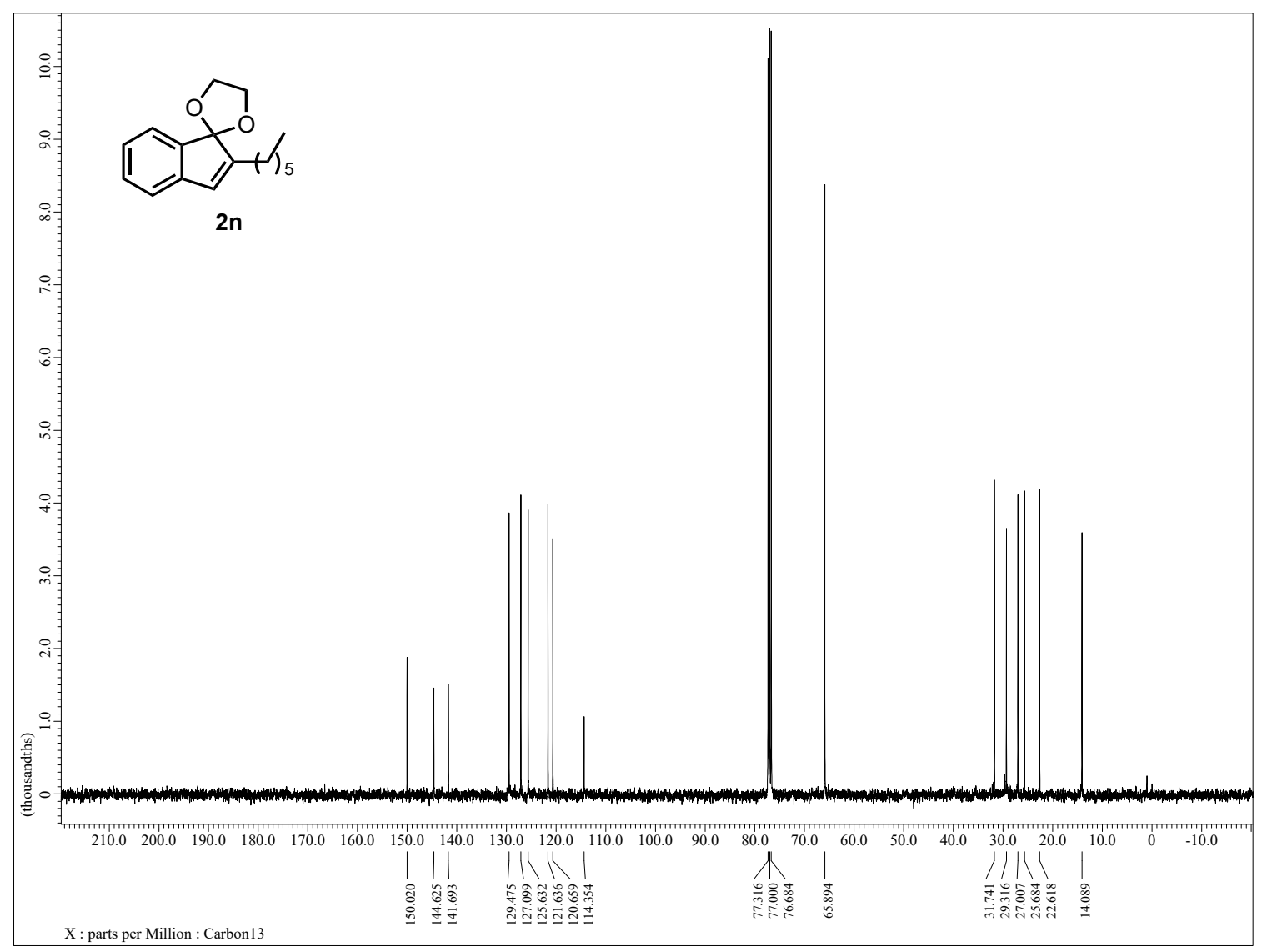


${ }^{1} \mathrm{H}$ NMR $\quad\left(400 \mathrm{MHz}, \mathrm{CDCl}_{3}\right)$ of $\mathbf{2 o}$

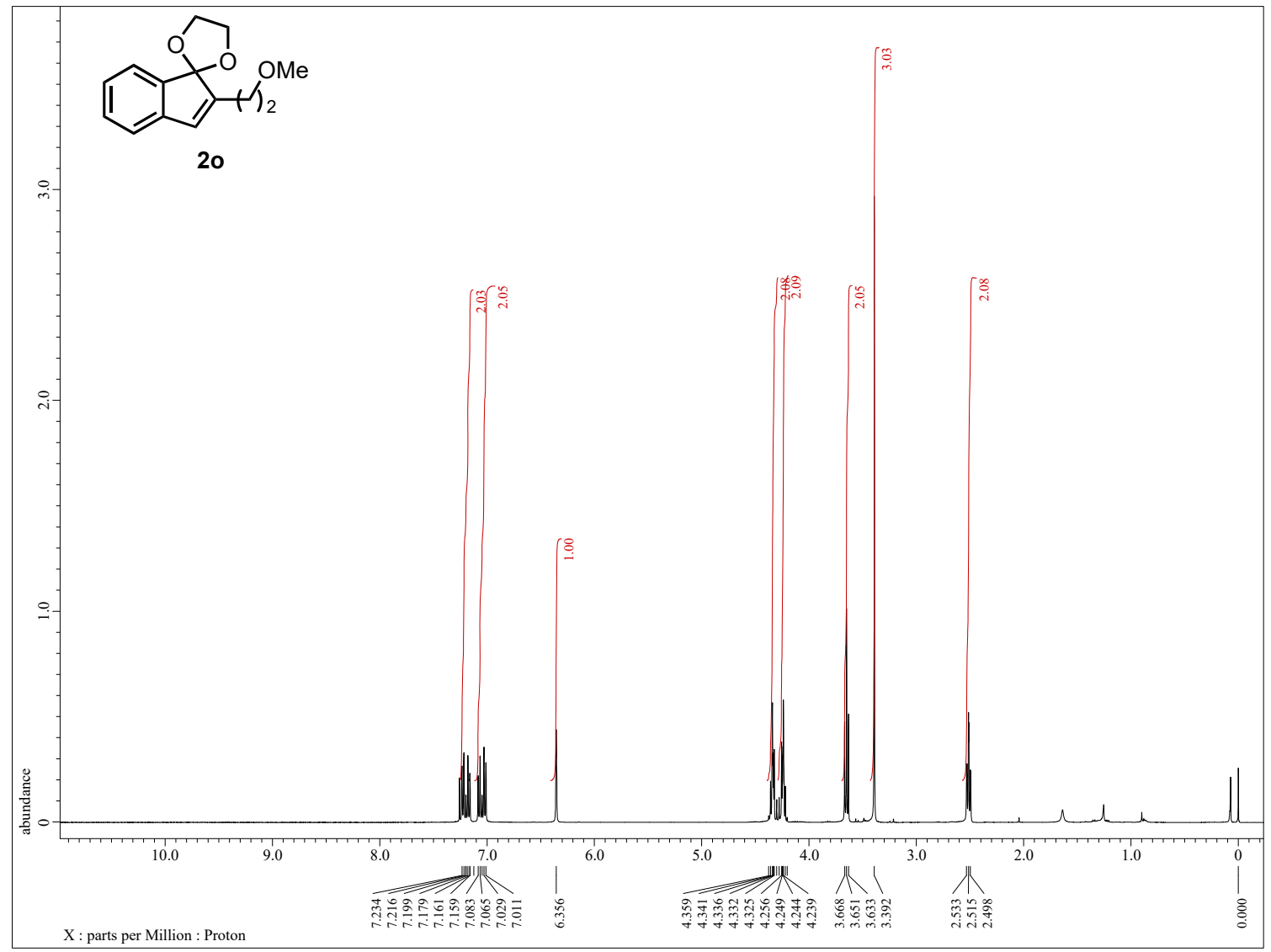

${ }^{13} \mathrm{C}$ NMR $\quad\left(100 \mathrm{MHz}, \mathrm{CDCl}_{3}\right)$ of $2 \mathbf{o}$

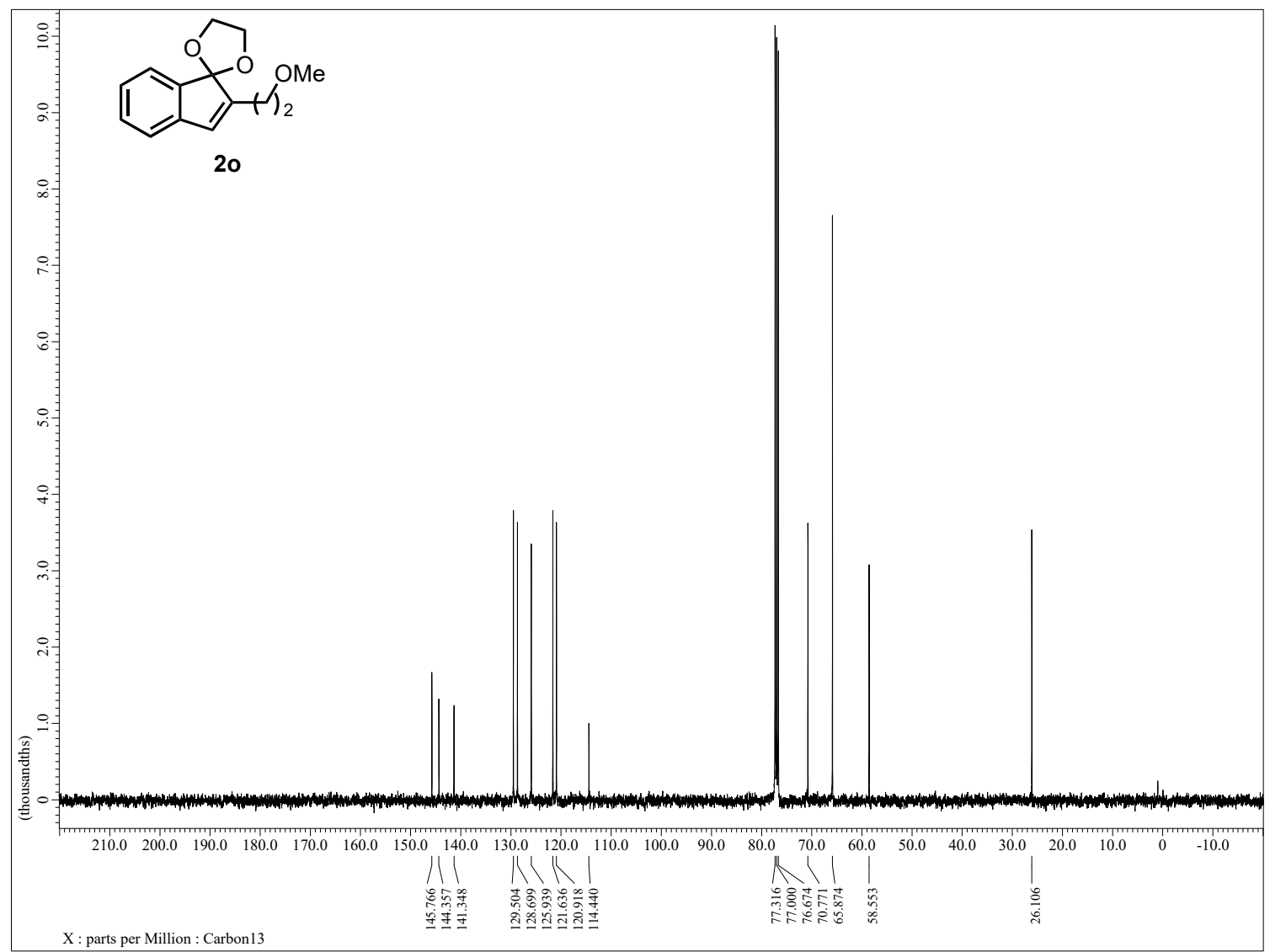


${ }^{1} \mathrm{H} \mathrm{NMR} \quad\left(500 \mathrm{MHz}, \mathrm{CDCl}_{3}\right)$ of $\mathbf{2 p}$

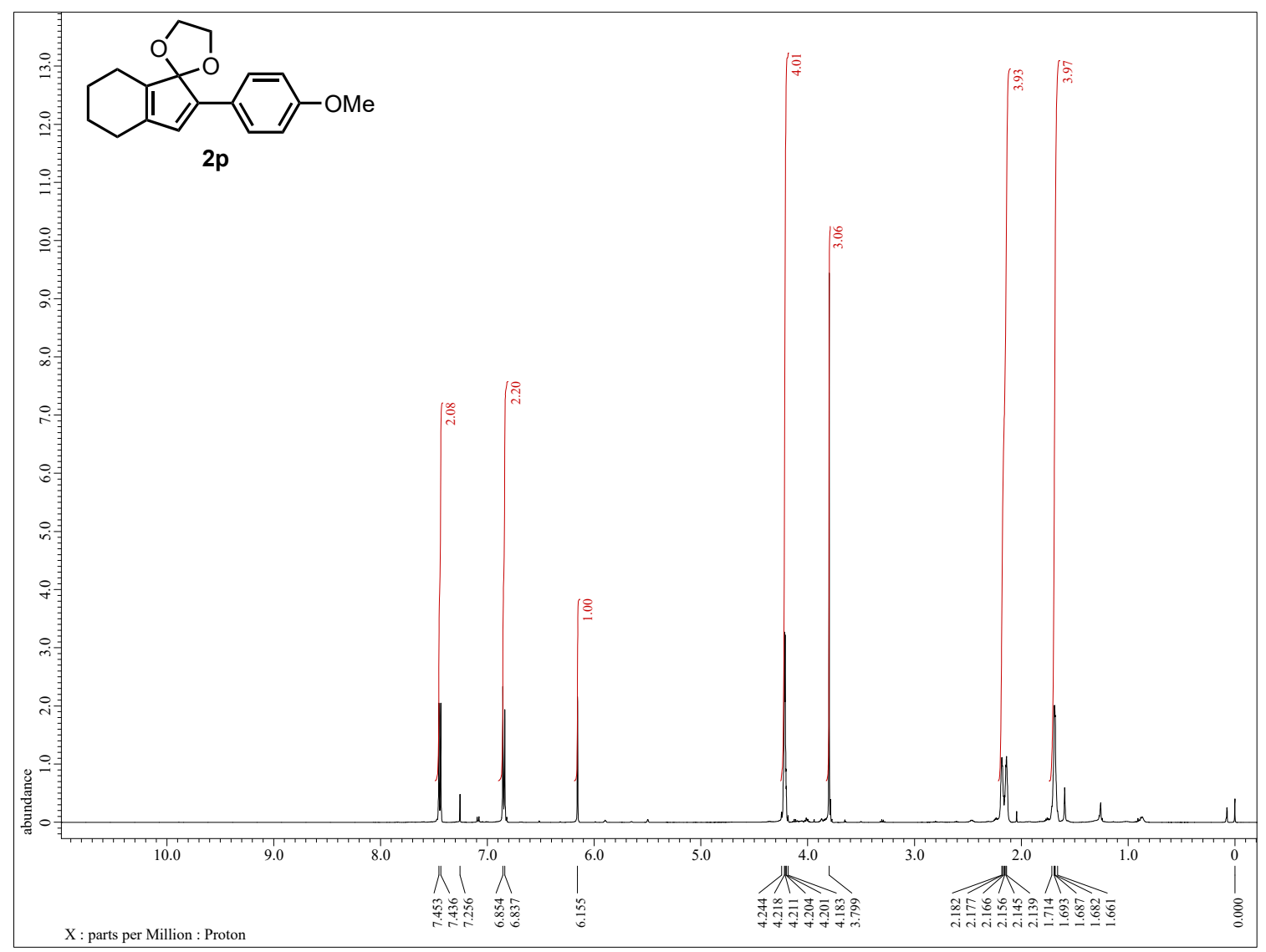

${ }^{13} \mathrm{C} \mathrm{NMR} \quad\left(125 \mathrm{MHz}, \mathrm{CDCl}_{3}\right)$ of $\mathbf{2 p}$

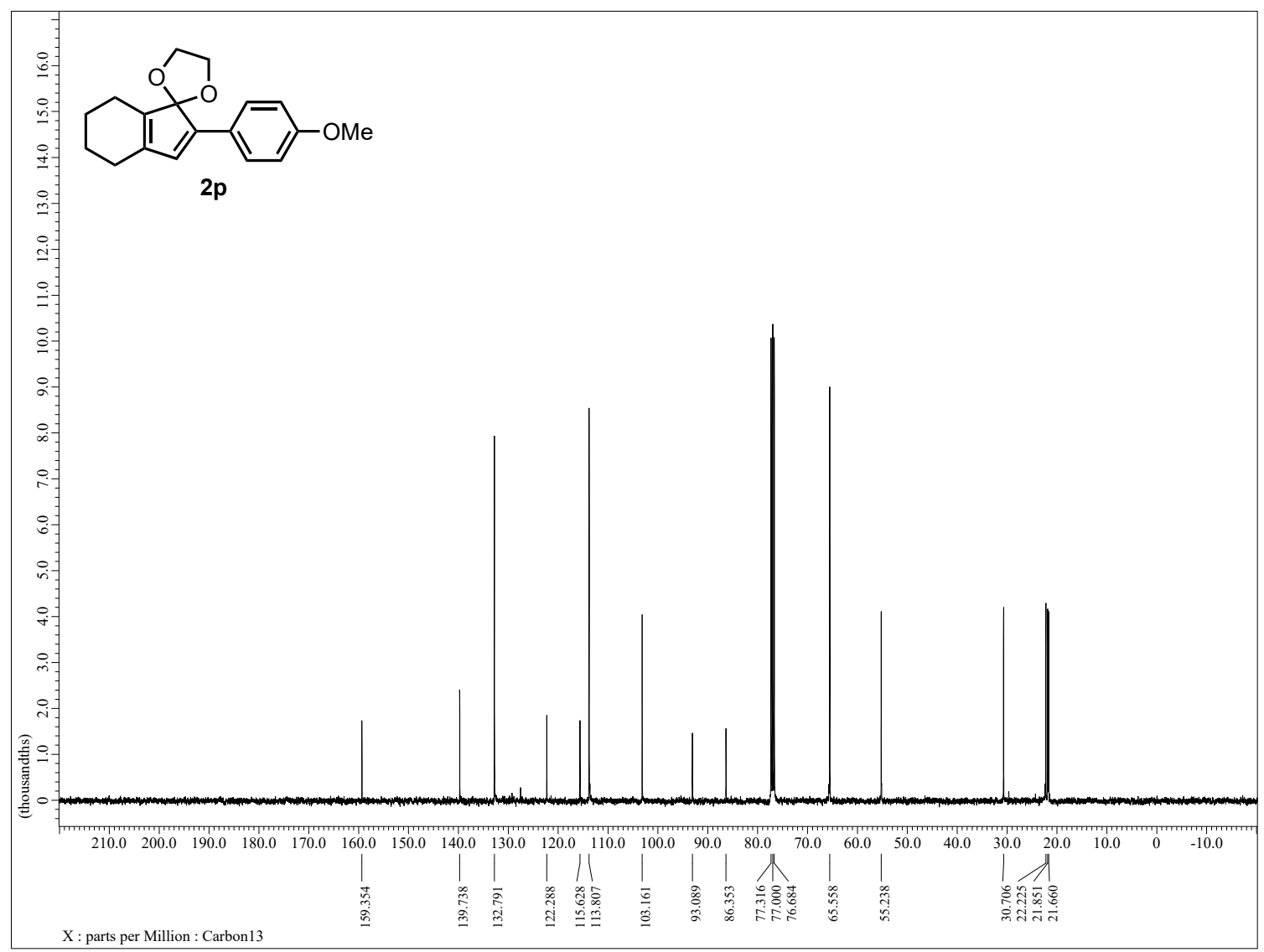


${ }^{1} \mathrm{H} \mathrm{NMR} \quad\left(400 \mathrm{MHz}, \mathrm{CDCl}_{3}\right)$ of $\mathbf{2 q}$

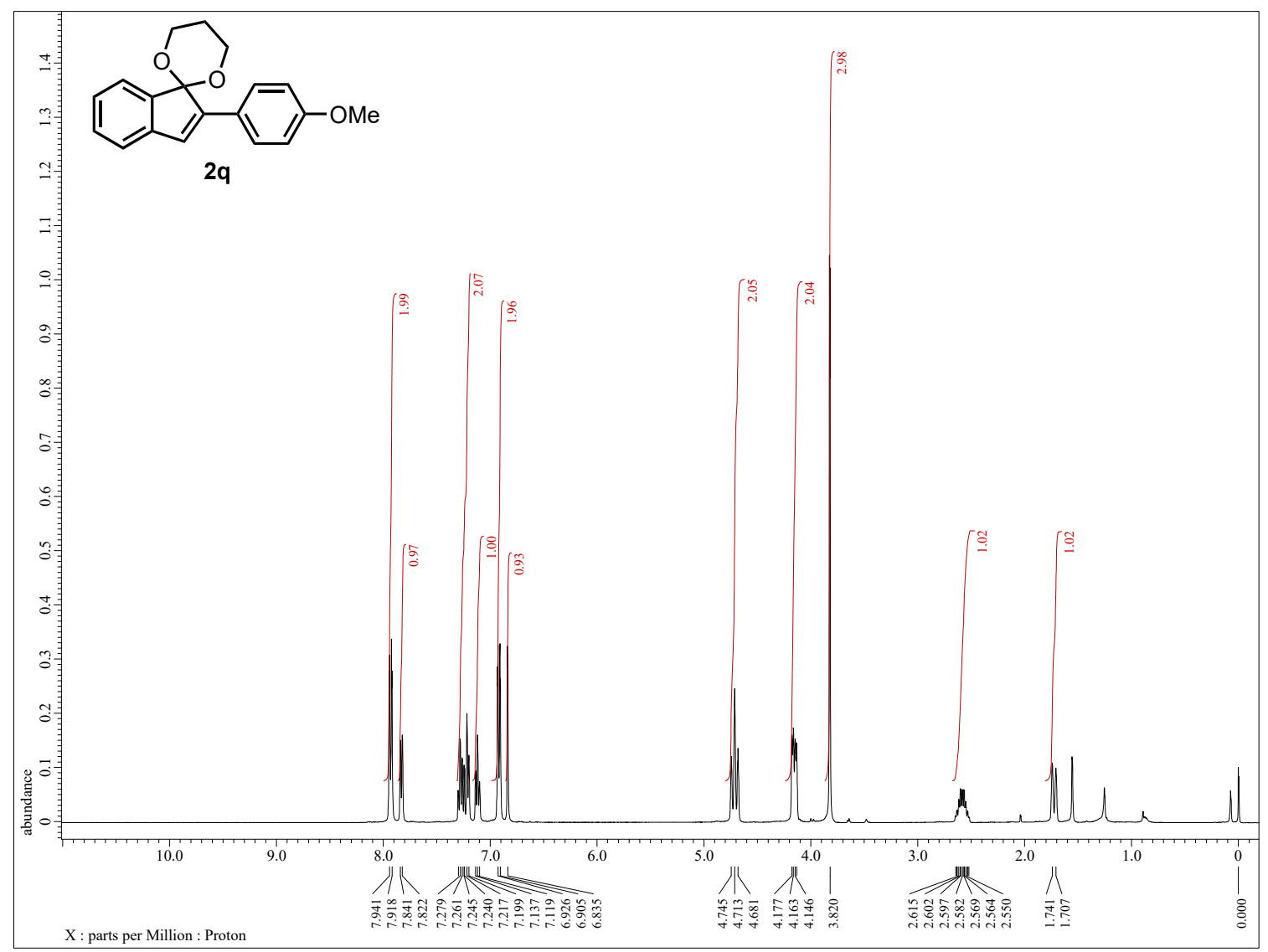

${ }^{13} \mathrm{C}$ NMR $\quad\left(100 \mathrm{MHz}, \mathrm{CDCl}_{3}\right)$ of $\mathbf{2 q}$

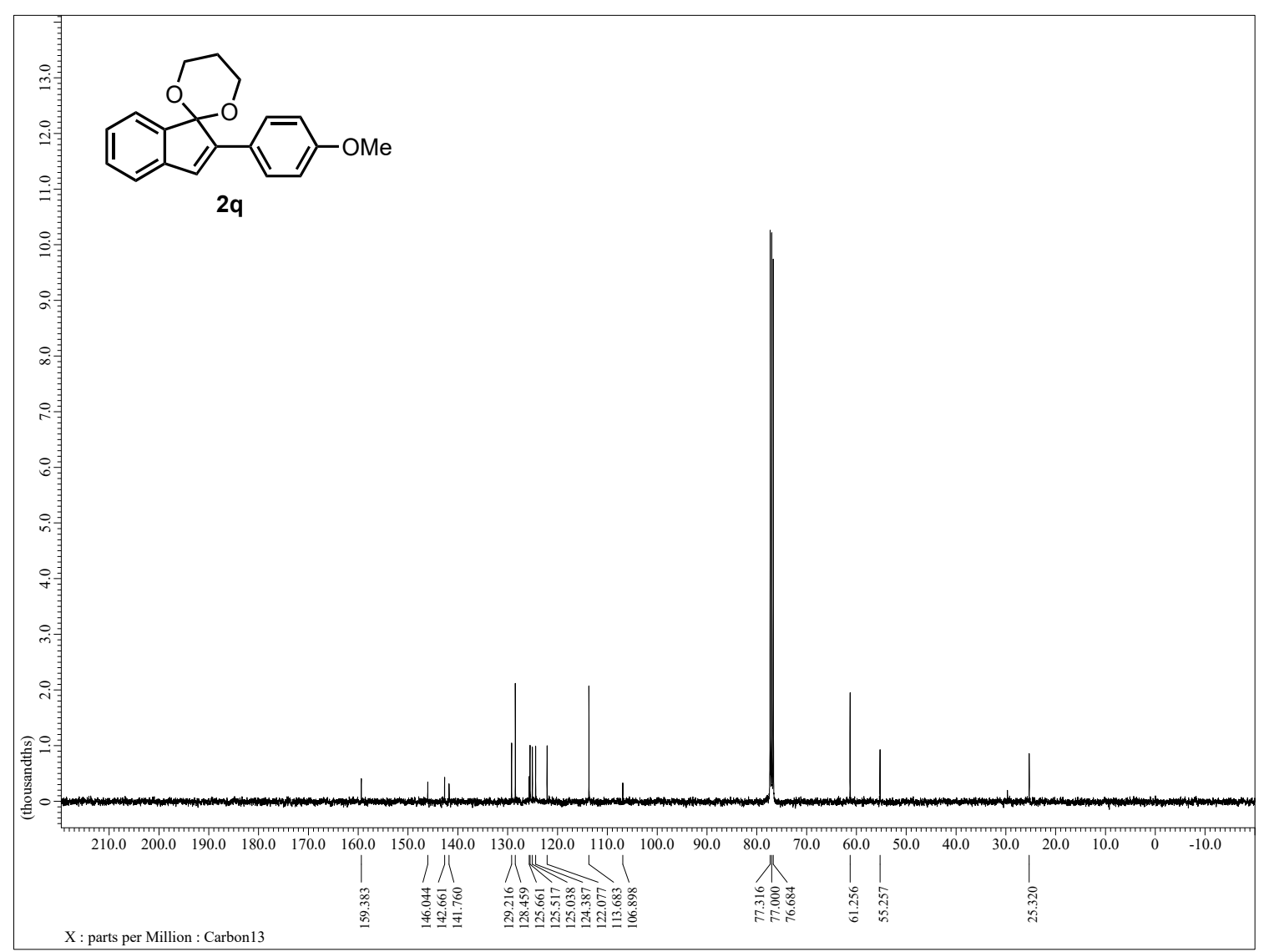


${ }^{1} \mathrm{H}$ NMR $\quad\left(400 \mathrm{MHz}, \mathrm{CDCl}_{3}\right)$ of $\mathbf{2 r}$

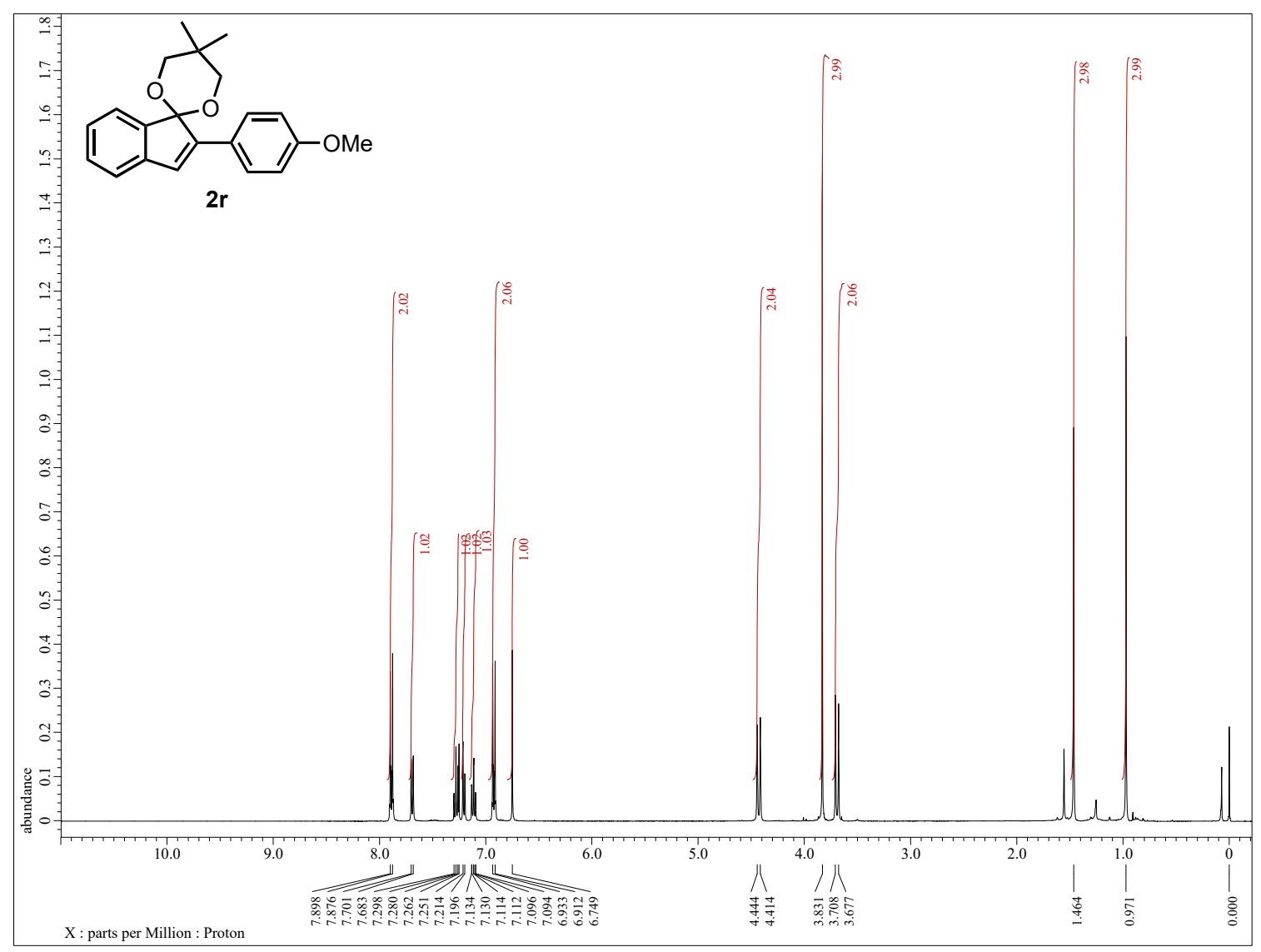

${ }^{13} \mathrm{C} \mathrm{NMR} \quad\left(100 \mathrm{MHz}, \mathrm{CDCl}_{3}\right)$ of $\mathbf{2 r}$

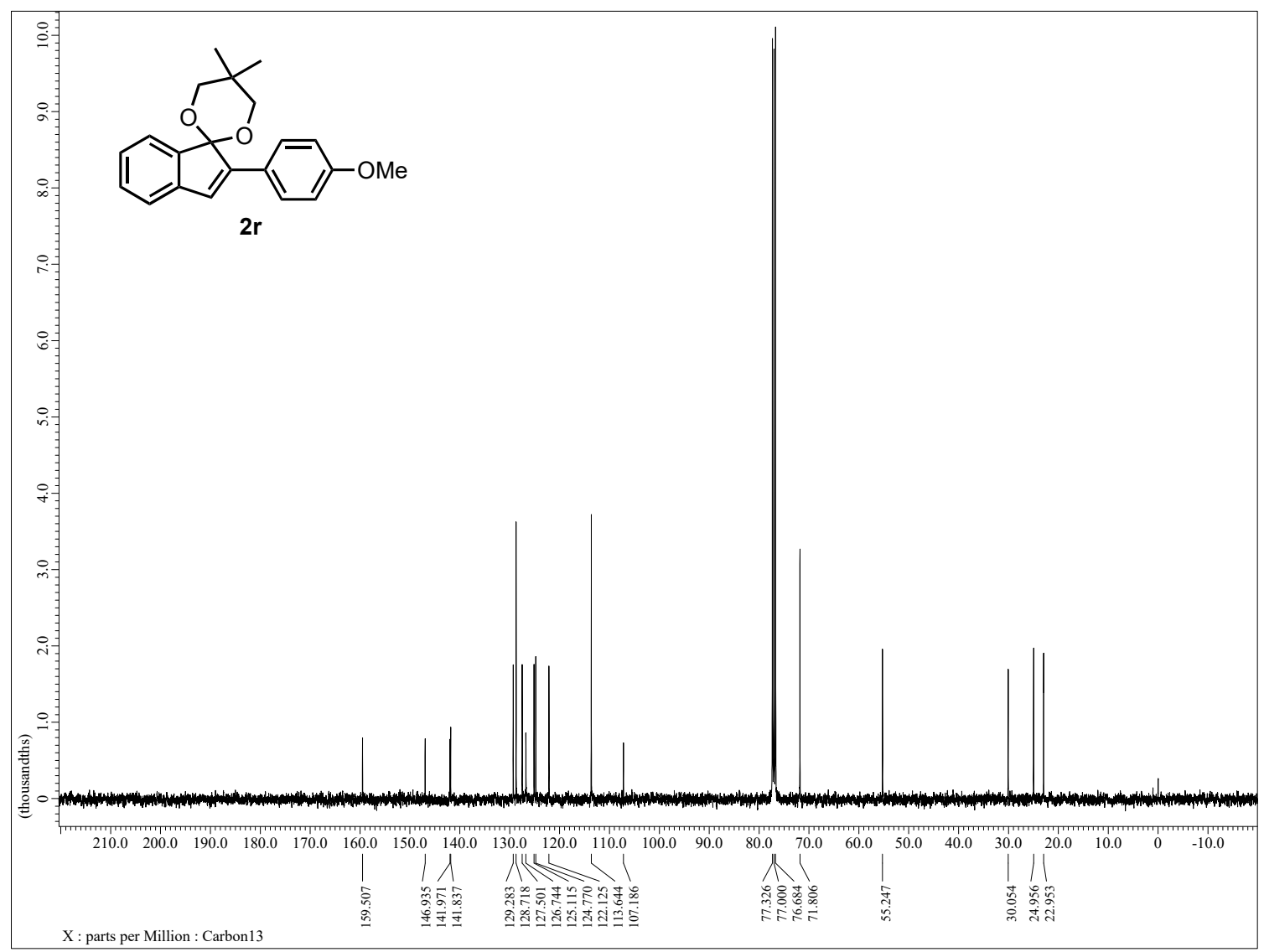


${ }^{1} \mathrm{H}$ NMR $\quad\left(400 \mathrm{MHz}, \mathrm{CDCl}_{3}\right)$ of $\mathbf{3 b}$

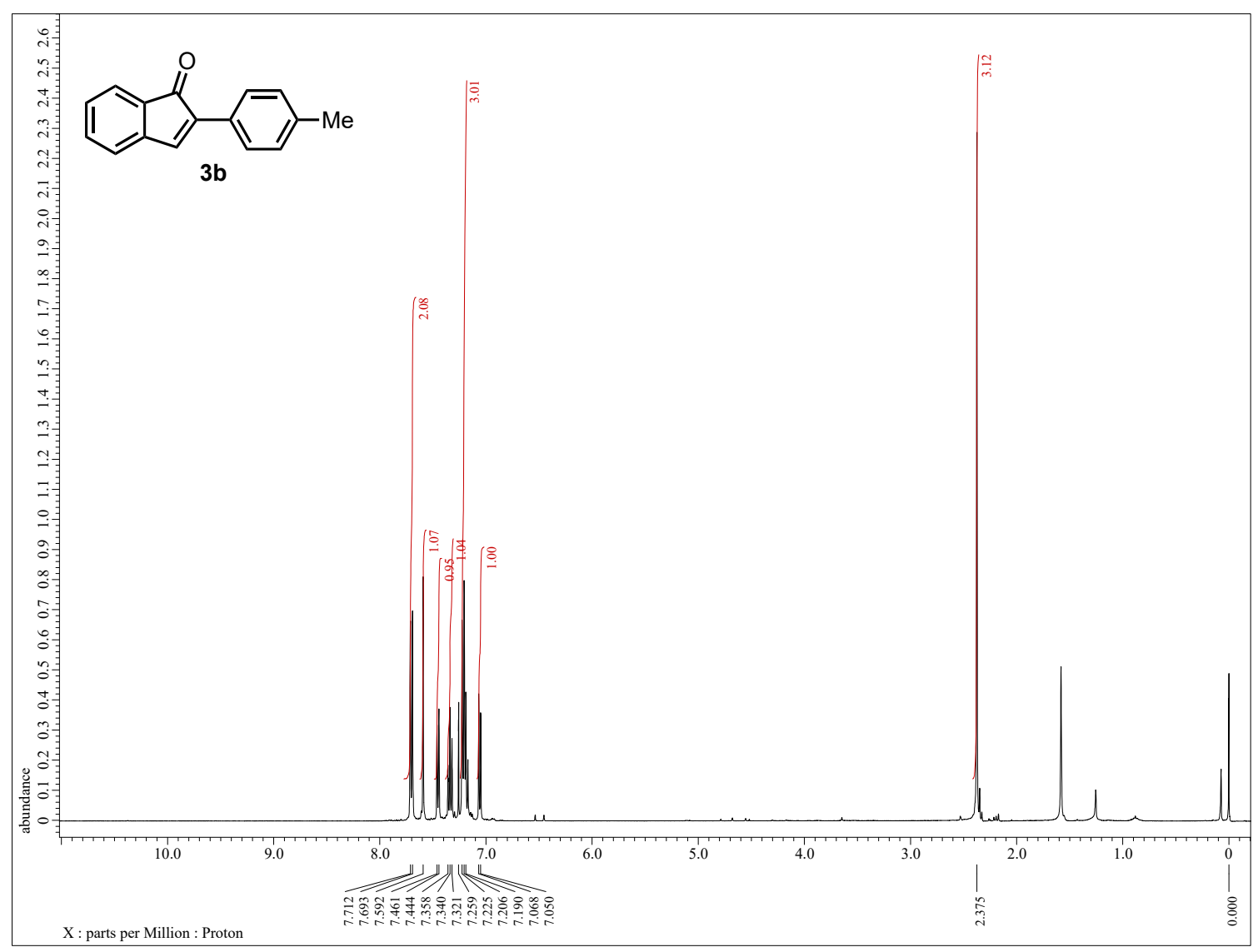

${ }^{13} \mathrm{C}$ NMR $\quad\left(100 \mathrm{MHz}, \mathrm{CDCl}_{3}\right)$ of $\mathbf{3 b}$

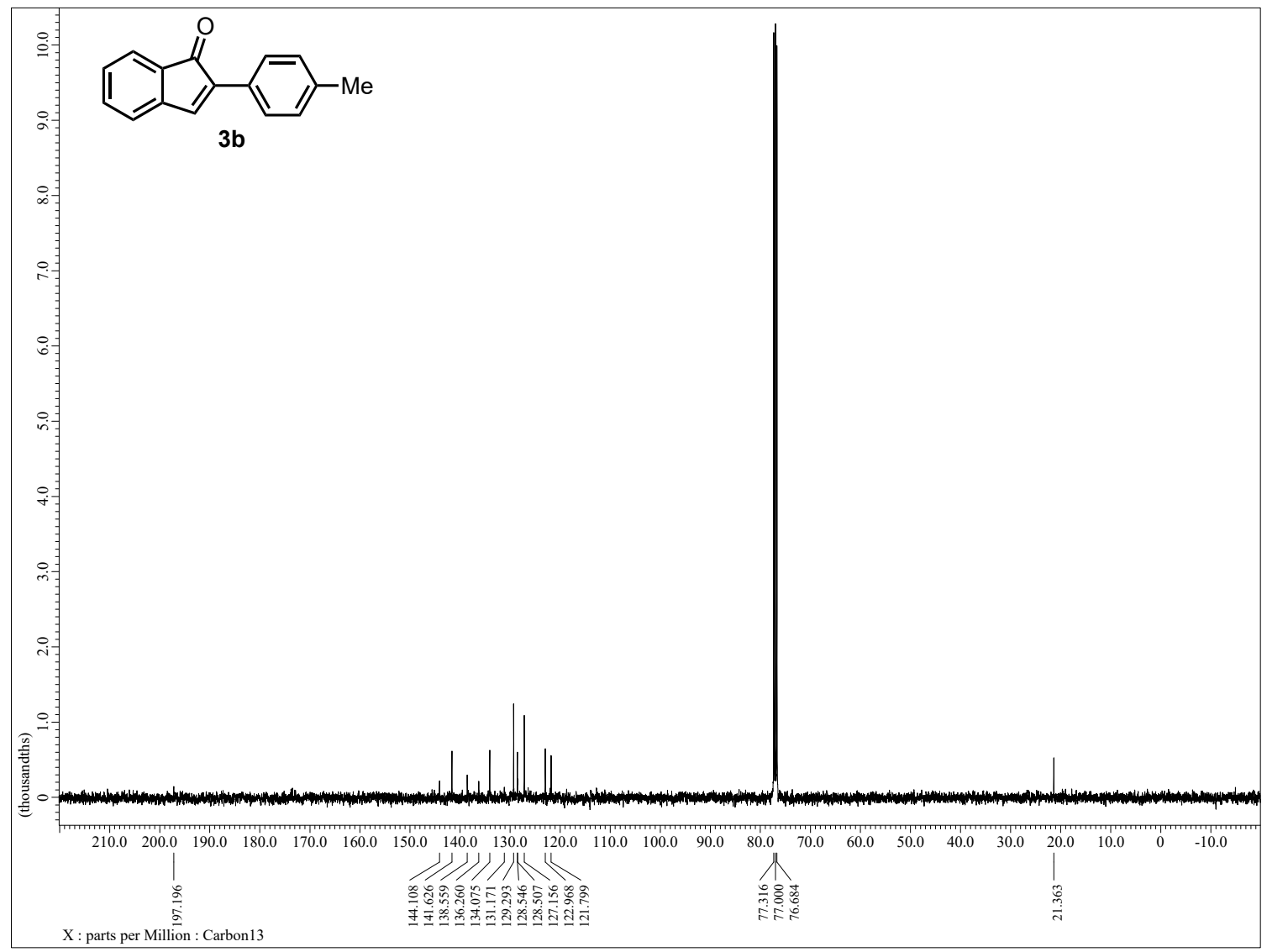


${ }^{1} \mathrm{H}$ NMR $\quad\left(400 \mathrm{MHz}, \mathrm{CDCl}_{3}\right)$ of 5

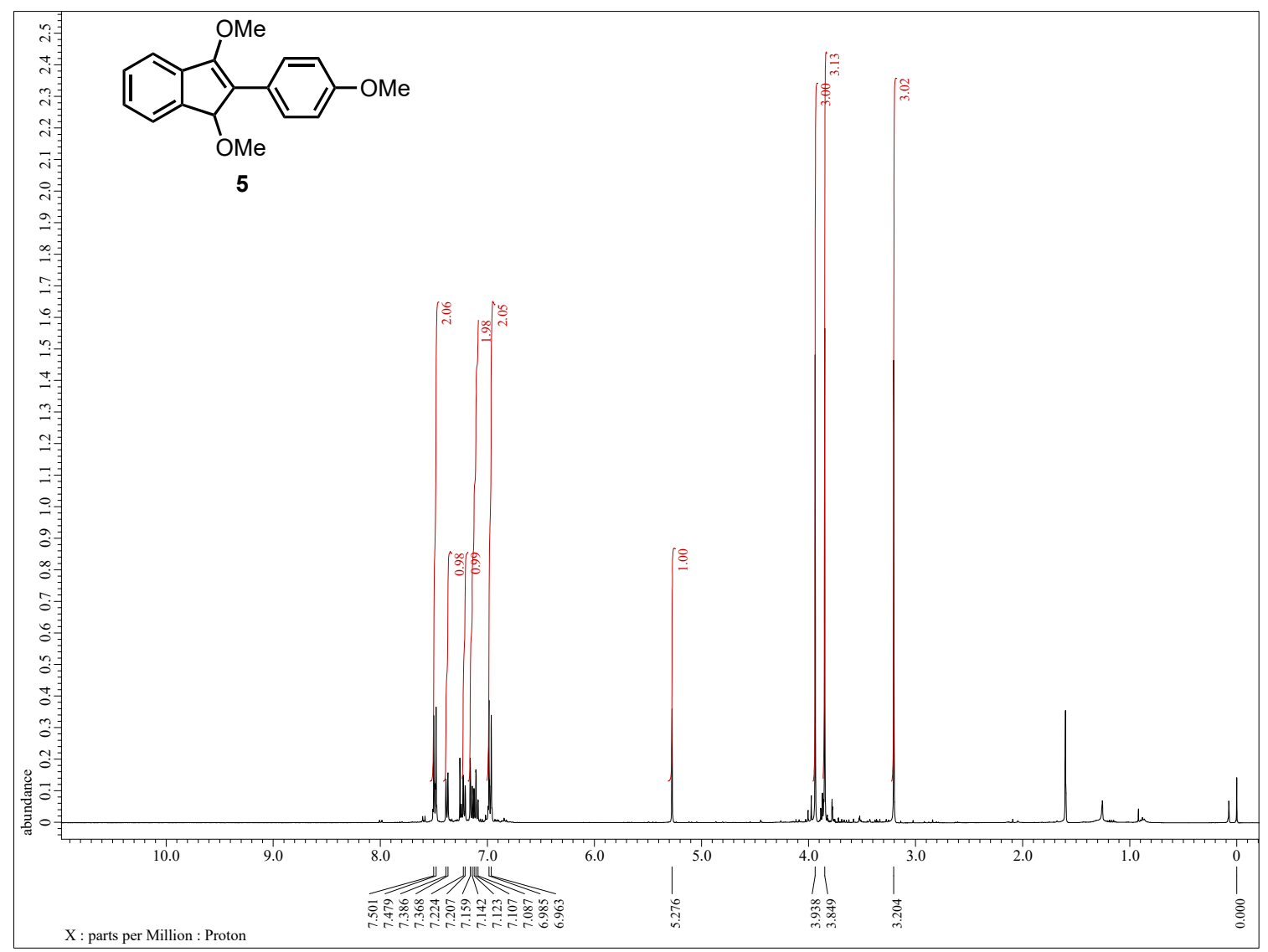

${ }^{13} \mathrm{C} \mathrm{NMR} \quad\left(100 \mathrm{MHz}, \mathrm{CDCl}_{3}\right)$ of $\mathbf{3 b}$

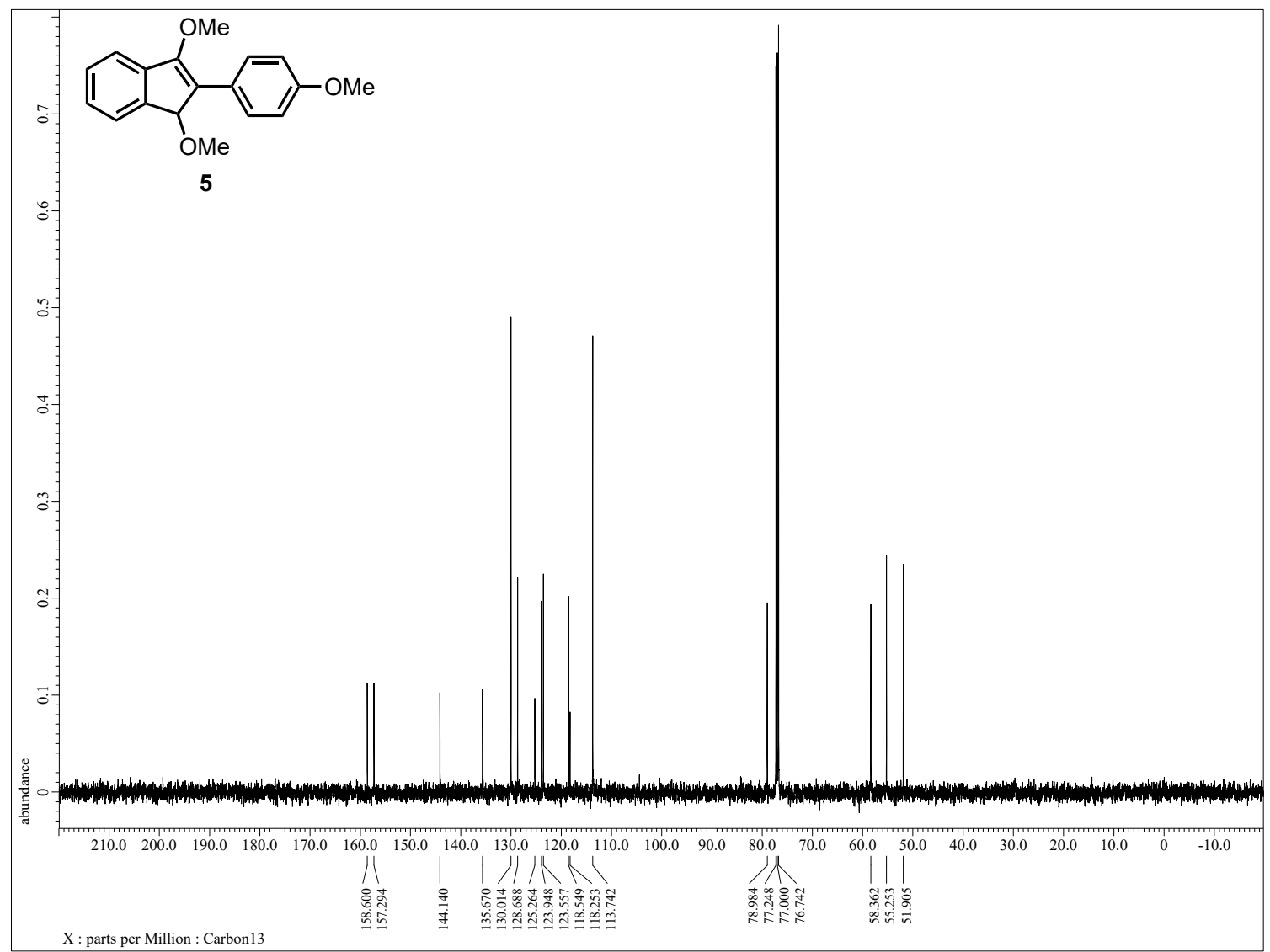


${ }^{1} \mathrm{H}$ NMR $\quad\left(400 \mathrm{MHz}, \mathrm{CDCl}_{3}\right)$ of $\mathbf{2} \mathbf{j}-\boldsymbol{d}_{\mathbf{1}}$

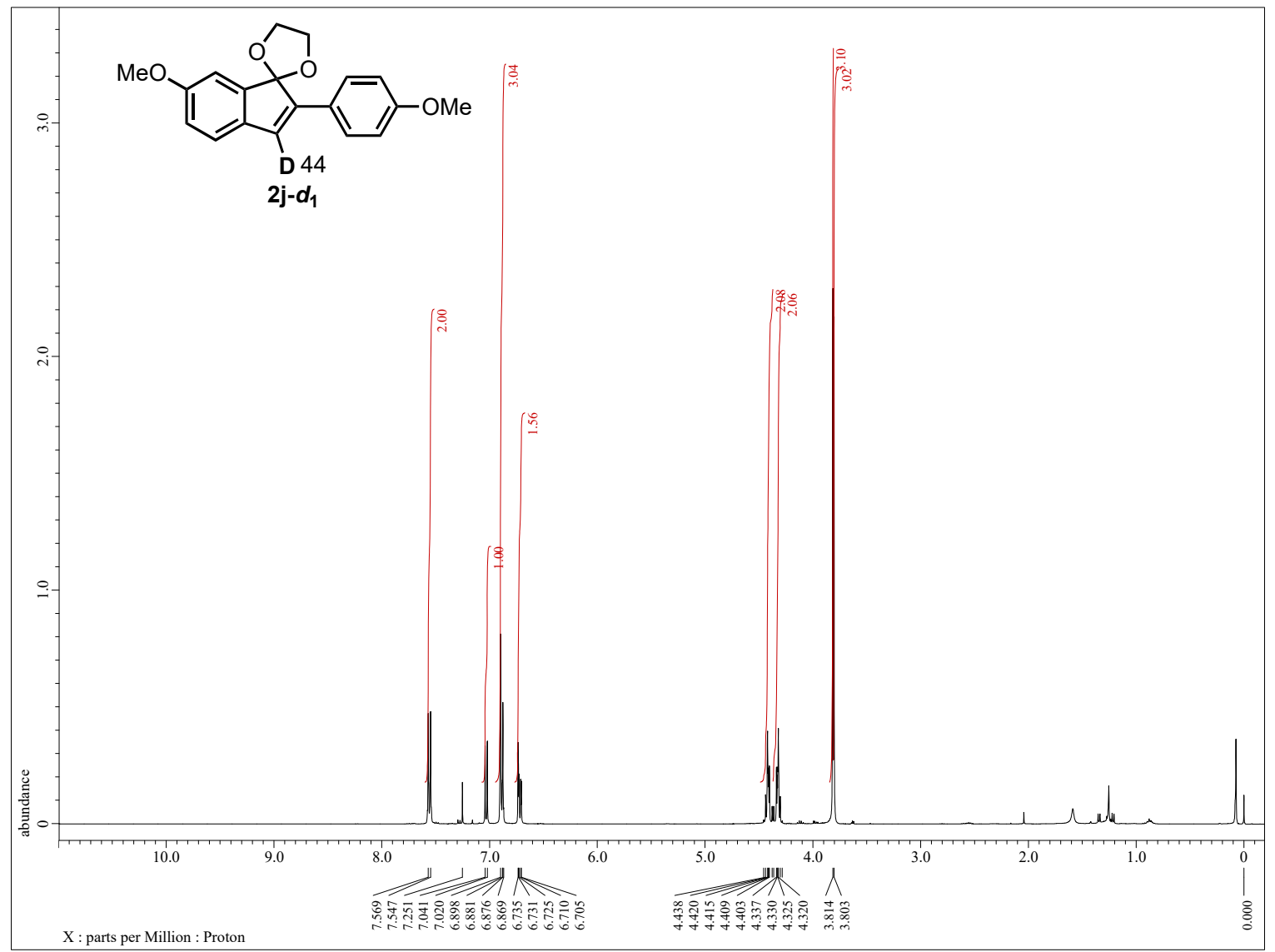

${ }^{2} \mathrm{H}$ NMR $\left(77 \mathrm{MHz}, \mathrm{CHCl}_{3}\right.$ ) of $\mathbf{2} \mathbf{j}-\boldsymbol{d}_{\mathbf{1}}$

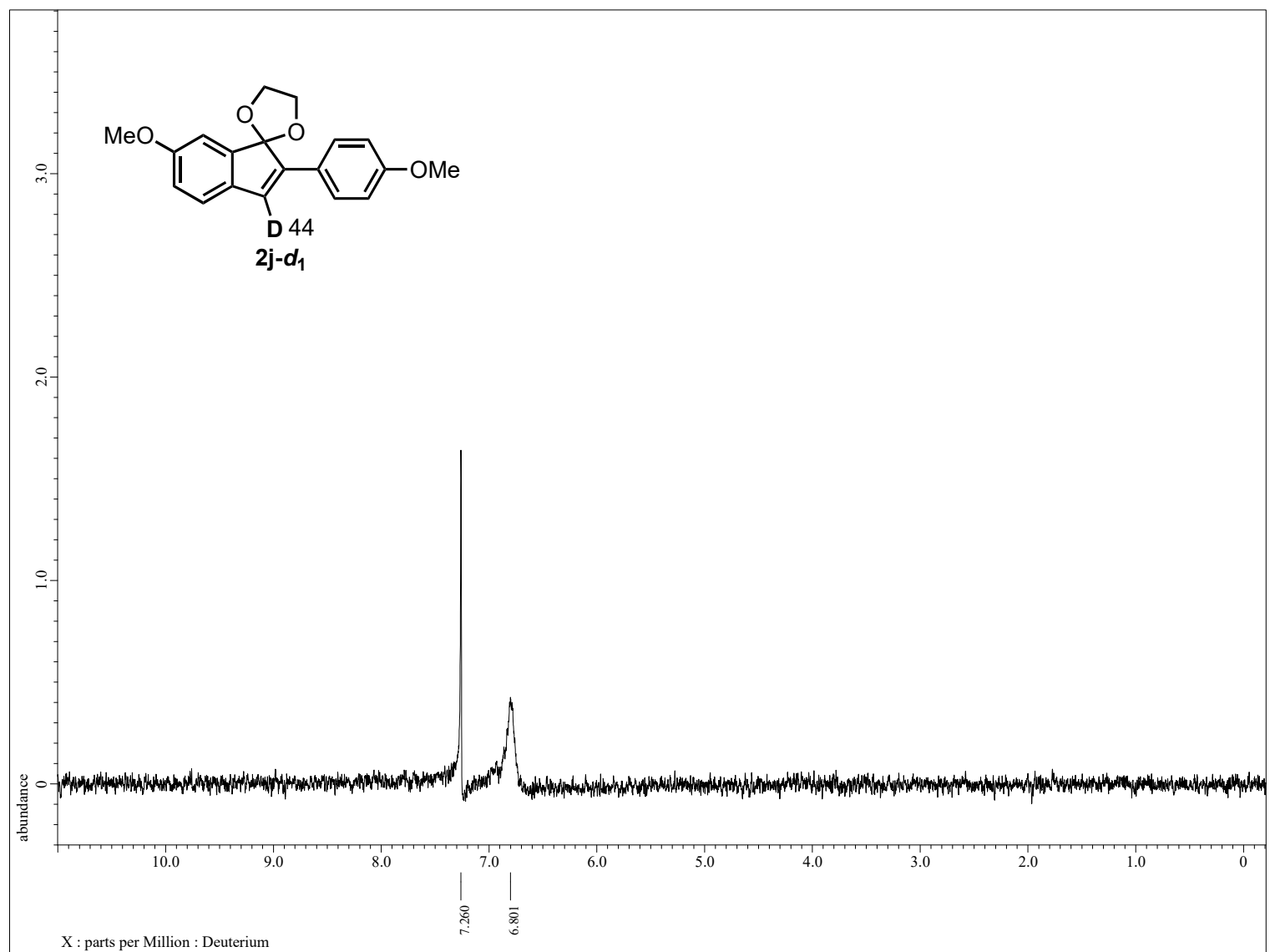


${ }^{1} \mathrm{H}$ NMR $\quad\left(400 \mathrm{MHz}, \mathrm{CDCl}_{3}\right)$ of $\mathbf{2 a}-\boldsymbol{d}_{\mathbf{1}}$

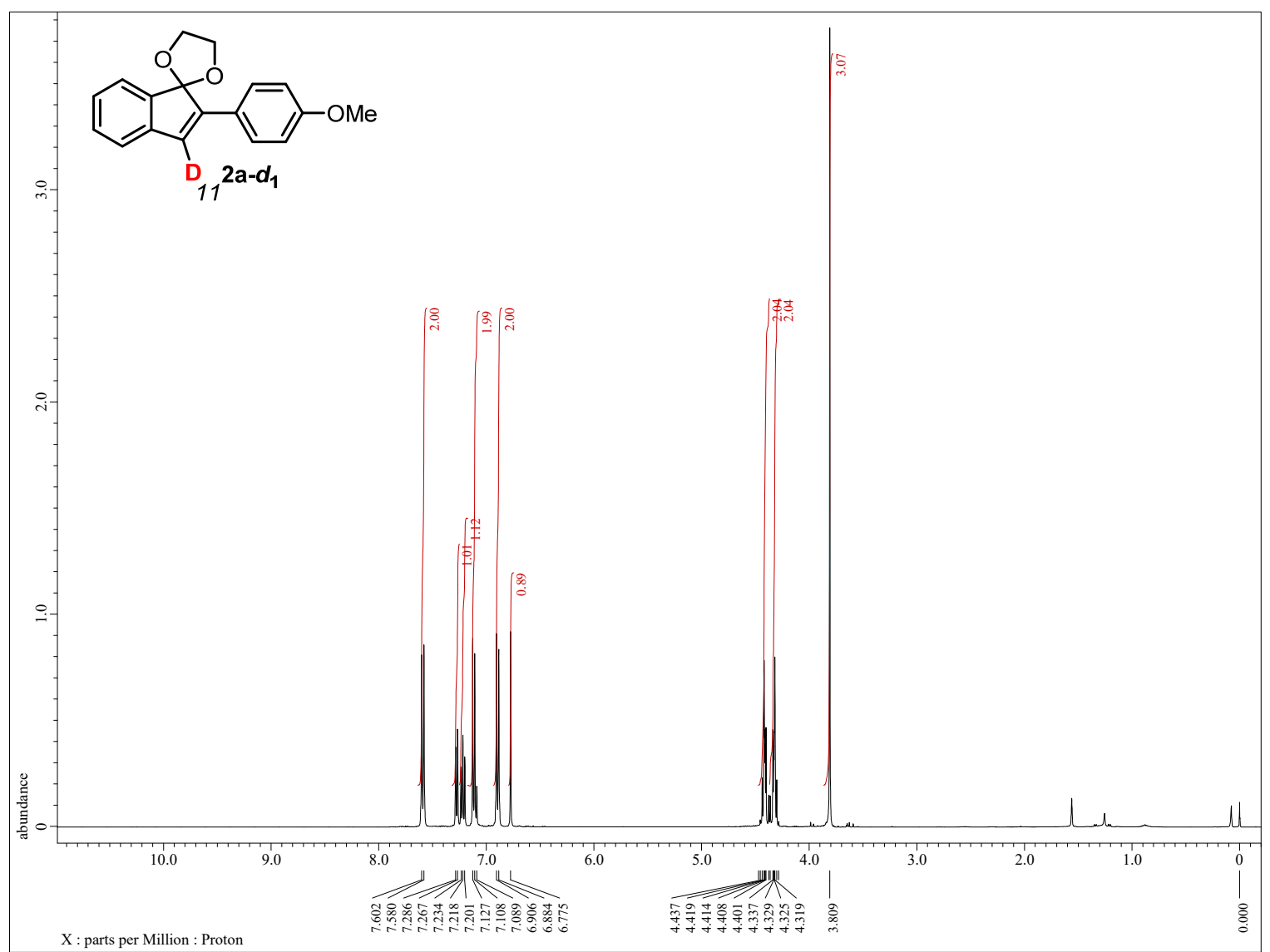

${ }^{2} \mathrm{H}$ NMR $\left(77 \mathrm{~Hz}, \mathrm{CHCl}_{3}\right.$ ) of $\mathbf{2 a - d _ { 1 }}$

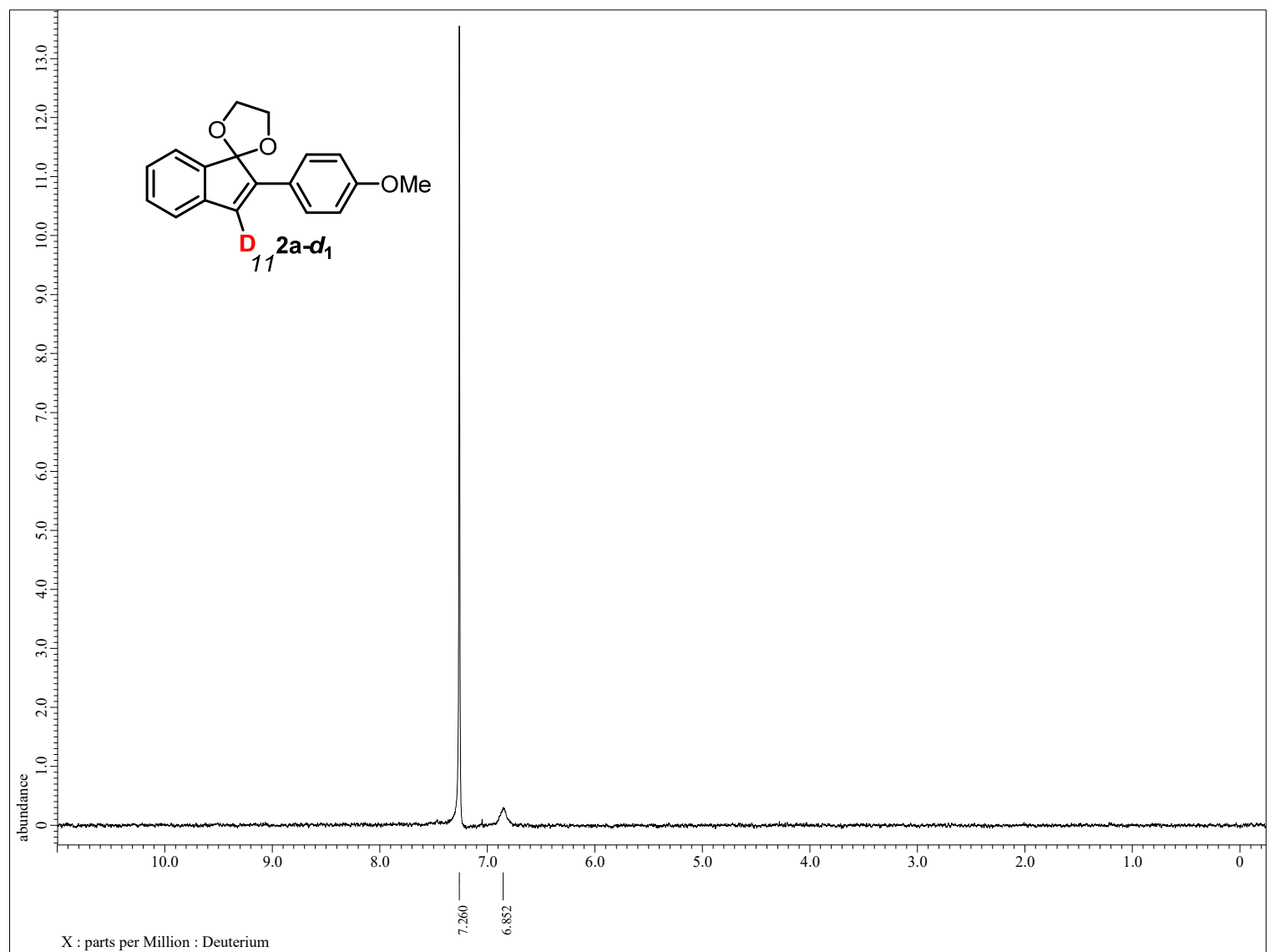




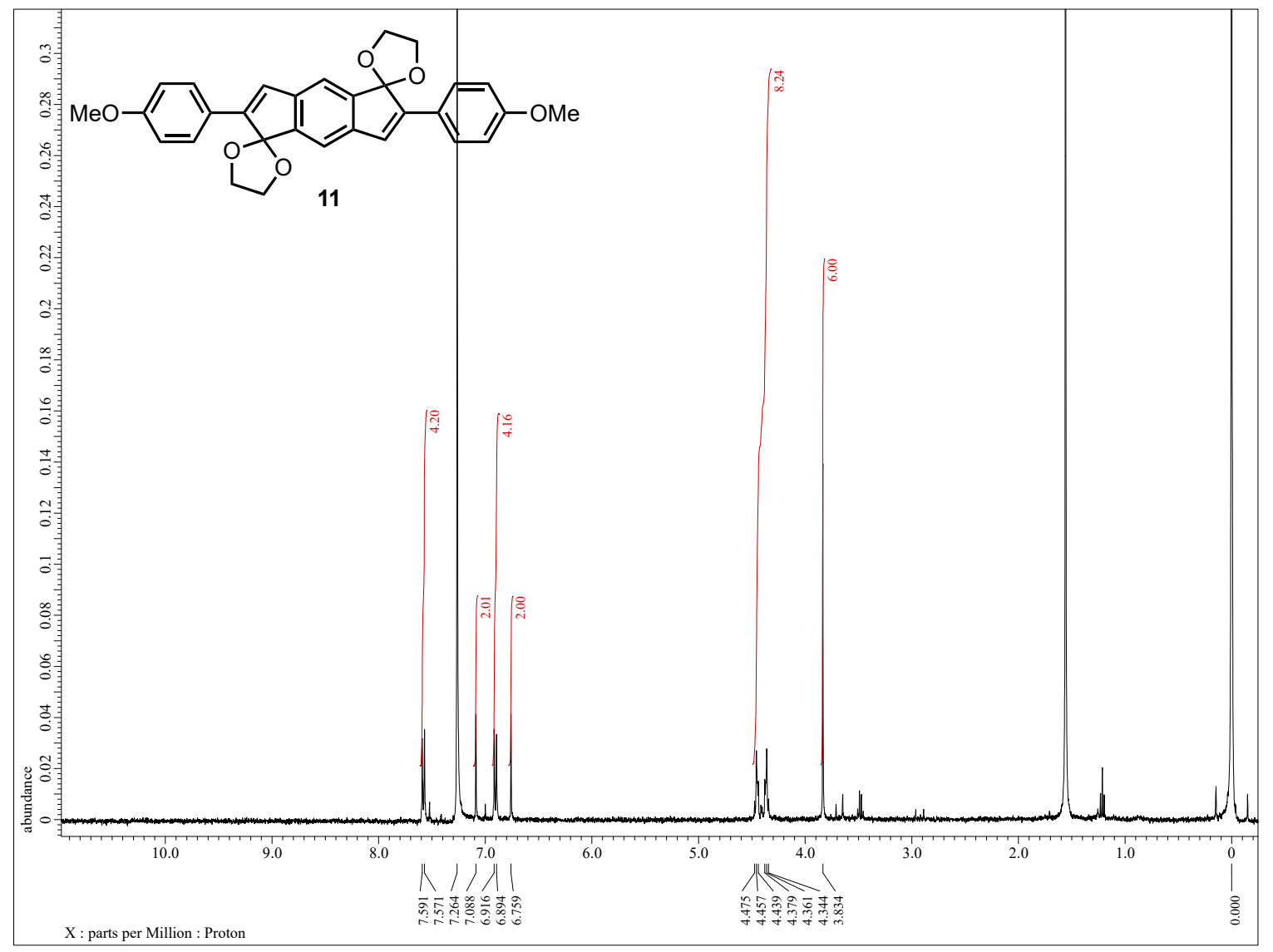

\section{References}

(1) Jian-Quan Liu, Xinyi Chen, Xuanyu Shen, Yihan Wang, Xiang-Shan Wang, Xihe Bi Adv. Synth. Catal. 2019, 361, $1543-1548$.

(2) Tian Feng, Yan He, Xinying Zhang, Xuesen Fan Adv. Synth. Catal. 2019, 361, 1271-1276.

(3) Akihiro Orita, Naonori Yoshioka, Petra Struwe, Arnold Braier, Anke Beckmann, Junzo Otera Chem. Eur. J. 1999, 5, $1355-1363$.

(4) Virsinha Reddy, Abhijeet S. Jadhav, Ramasamy Vijaya Anand Org. Biomol. Chem. 2015, 13, 3732-3741.

(5) Jia Wang, Hai-Tao Zhu, Ying-Xiu Li, Li-Jing Wang, Yi-Feng Qiu, Zi-Hang Qiu, Mei-jin Zhong, Xue-Yuan Liu, Yong-Min Liang Org. Lett. 2014, 16, 2236-2239.

(6) Felix Scheidt, Michel Schäfer, Jéróme C. Sarie, Constanin G. Daniliuc, John J. Molloy, Ryan Gilmour Angew. Chem. Int. Ed. 2018, 57, 16431-16435.

(7) Vibha Tandon, Urvashi, Pooja Yadav, Souvik Sur, Sheenu Abbat, Vinod Tiwari, Raymond Kukreti, Maria A. Papathanasopoulos, Rameez Raja, Akhil C. Banerjea, Akhilesh K. Verma, Shrikant Kukreti, Prasad V. Bharatam ACS Med. Chem. Lett. 2015, 6, 1065-1070.

(8) Rahulkumar Rajmani Singh, Manisha Skaria, Liang-Yu Chen, Mu-Jeng Cheng, Rai-Shung Liu Chem. Sci. 2019, 10, 1201-1206.

(9) Lun Wang, Lingyan Liu, Weixing Chang, Jing Li J. Org. Chem. 2018, 83, 7799-7813.

(10) Monica Dell’ Acqua, Diego Facoetti, Giorgio Abbiati, Elisabetta Rossi Synthesis 2010, 14, 2367-2378. 
(11) Zheliang Yuan, Ran Cheng, Pinhong Chen, Guosheng Liu, Steven H. Liang Angew. Chem. Int. Ed. 2016, 55, $11882-11886$.

(12) Jintao Zhang, Yelin Xiao, Kai Chen, Wanqing Wu, Huanheng Jiang, Shifa Zhu Adv. Synth. Catal. 2016, 358, 2684-2691.

(13) Jia Wang, Hai-Tao Zhu, Si Chen, Cheng Luan, Yu Xia, Yi Shen, Ying-Xiu Li, Yingxi Hua, Yong-Min Liang J. Org. Chem. 2017, 82, 10641-10649.

(14) Dirk Hildebrandt, Wiebke Hüggenberg, Matthias Kanthak, Tobias Plöger, Iris M. Müller, Gerald Dyker Chem. Commun. 2006, 2260-2261.

(15) Shasha Zheng, Gu Lingyue, Michelle Jui Hsien Ong, Denis Jacquemin, Anthony Romieu, Jean-Alexandre Richard, Rajavel Srinivasan Org. Biomol. Chem. 2019, 17, 4291-4300.

(16) Ana M. Sanjuán, Patricia García-García, Manuel A. Fernández-Rodríguez, Roberto Sanz Adv. Synth. Catal. 2013, 355, $1955-1962$.

(17) Xiao-Wei Liu, Shu-Sen Li, Dong-Ting Dai, Meng Zhao, Cui-Cui Shan, Yun-He Xu, Teck-Peng Loh Org. Lett. 2019, 21, 3696-3700.

(18) Pablo Morán-Poladura, Eduarbo Rubio, José M. González Angew. Chem. Int. Ed. 2015, 54, 3052-3055.

(19) Yanbin Hu, Yanan Li, Sheng Zhang, Chong Li, Lijun Li, Zhenggen Zha, Zhiyong Wang Org. Lett. 2015, 17, 4018-4021.

(20) Milan Arambasic, Manjeet K. Majhail, Robert. N. Straker, James D. Neuhaus, Michel C. Wills Chem. Commun. 2019, $55,2757-2760$.

(21) Vanessa Claus, Michael Schulin, Siegfried Harrer, Matthias Rudolph, Frank Rominger, Abdullah M. Asiri, Jin Xie, A. Stephen K. Hashmi Angew. Chem. In. Ed. 2018, 57, 12966-12970.

(22) Natalia Prusinowska, Matuesz Bardziński, Agnieszka Janiak, Pawel Skowronek, Marcin Kwit Chem. Asian J. 2018, 13, 2691-2699. 